\title{
Performance Bounds on Matched-Field Methods for Source Localization and Estimation of Ocean Environmental Parameters
}

\author{
by \\ Wen Xu
}

B.E., Electrical Engineering, University of Science and Technology of China, 1990

M.S., Acoustics, Institute of Acoustics, Chinese Academy of Sciences, 1993

M.S., Oceanographic Engineering, MIT/WHOI Joint Program, 1998

Submitted to the Department of Ocean Engineering of MIT and

the Department of Applied Ocean Physics and Engineering of WHOI in partial fulfillment of the requirements for the degree of

Doctor of Philosophy in Oceanographic Engineering

at the

MASSACHUSETTS INSTITUTE OF TECHNOLOGY

and the

WOODS HOLE OCEANOGRAPHIC INSTITUTION

June 2001

(C) Massachusetts Institute of Technology and Woods Hole Oceanographic

Institution, 2001. All rights reserver 


\title{
Performance Bounds on Matched-Field Methods for Source Localization and Estimation of Ocean Environmental

\author{
Parameters
}

by

Wen Xu

\begin{abstract}
Submitted to the Department of Ocean Engineering of MIT and

the Department of Applied Ocean Physics and Engineering of WHOI on May 24, 2001, in partial fulfillment of the requirements for the degree of Doctor of Philosophy in Oceanographic Engineering
\end{abstract}

\begin{abstract}
Matched-field methods concern estimation of source location and/or ocean environmental parameters by exploiting full wave modeling of acoustic waveguide propagation. Typical estimation performance demonstrates two fundamental limitations. First, sidelobe ambiguities dominate the estimation at low signal-to-noise ratio (SNR), leading to a threshold performance behavior. Second, most matched-field algorithms show a strong sensitivity to environmental/system mismatch, introducing some biased estimates at high SNR.

In this thesis, a quantitative approach for ambiguity analysis is developed so that different mainlobe and sidelobe error contributions can be compared at different SNR levels. Two large-error performance bounds, the Weiss-Weinstein bound (WWB) and Ziv-Zakai bound (ZZB), are derived for the attainable accuracy of matched-field methods. To include mismatch effects, a modified version of the ZZB is proposed.

Performance analyses are implemented for source localization under a typical shallow water environment chosen from the Shallow Water Evaluation Cell Experiments (SWellEX). The performance predictions describe the simulations of the maximum likelihood estimator (MLE) well, including the mean square error in all SNR regions as well as the bias at high SNR. The threshold SNR and bias predictions are also verified by the SWellEX experimental data processing. These developments provide tools to better understand some fundamental behaviors in matched-field performance and provide benchmarks to which various ad hoc algorithms can be compared.
\end{abstract}

Thesis Supervisor: Arthur B. Baggeroer

Title: Ford Professor of Engineering

Secretary of the Navy / Chief of Naval Operations Chair for Ocean Science 


\section{Acknowledgments}

I am deeply grateful to my thesis supervisor, Prof. Arthur Baggeroer, who provided me this unique opportunity and guided me throughout the thesis. I couldn't complete it without his insight and confidence on this subject. I would also like to thank the other members on my thesis committee: Prof. Henrik Schmidt, Dr. George Frisk, and Prof. John Leonard, for their valuable guidance as well as continuous encouragement.

I would like to specially thank Prof. Kristine Bell of GMU, who also served on my thesis committee, and Dr. Christ Richmond of Lincoln Lab. They directly contributed to the theoretical developments in this thesis. I would also like to thank Dr. James Preisig and Prof. Nick Makris, for valuable discussions on my research.

I would like to specially thank Mr. Phil Schey of SPAWAR San Diego, who provided the SWellEX experimental data and was always open to my questions. I would also like to thank Mr. Paul Baxley of SPAWAR, Prof. William Hodgkiss and Prof. William Kuperman of SIO for their help in experimental data processing.

I would like to thank my officemates at the MIT Ocean Acoustics Group for their valuable help, particularly, Dr. Aaron Thode, Dr. Peter Daly, and Ms. Sabina Rataj; others including Yi-san Lai, Geoff Fox, Kyle Becker, Purnima Ratilal, Jennifer Munro, Luiz Souza, Te-Chih Liu, Joshua Wilson, and Michele Zanolin.

I would like to thank Prof. Chryssostomos Chryssostomidis, Dr. John Farrington, Dr. Mark Grossenbaugh, and Prof. Paola Rizzoli, for their support to my doctoral program. I would also like to thank the MIT OE Administration Office and the WHOI Education Office, who assisted me in many ways during this work.

I am specially grateful to Dr. Ken Stewart, who guided my master thesis here. I would also like to thank my friends, Yanwu Zhang, Leslie Loo, Marilyn Chen, Spencer and Leslie Stith, Xiaoou Tang, and Dezhang Chu, for all kinds of help.

Finally, and most importantly, I would like to thank my wife Hannah, my parents, sisters, and in-laws, for their love, encouragement, and support.

Financial support for my research was provided by the Office of Naval Research and the WHOI Education Office. 


\section{Abbreviations}

$\begin{array}{ll}\text { CRB } & \text { Cramer-Rao Bound } \\ \text { DGPS } & \text { Differential Global Positioning System } \\ \text { MAP } & \text { Maximum A Posteriori Estimator } \\ \text { MFP } & \text { Matched-Field Processing } \\ \text { MFT } & \text { Matched-Field Tomography } \\ \text { MLE } & \text { Maximum Likelihood Estimator } \\ \text { MMSE } & \text { Minimum Mean Square Error Estimator } \\ \text { MSE } & \text { Mean Square Error } \\ \text { MZZB } & \text { Modified Ziv-Zakai Bound } \\ \text { SNR } & \text { Signal-to-Noise Ratio } \\ \text { WWB } & \text { Weiss-Weinstein Bound } \\ \text { ZZB } & \text { Ziv-Zakai Bound } \\ \text { pdf } & \text { probability density function }\end{array}$




\section{Notation}

In this thesis, scalar variables are represented in italics $(r)$, vector quantities are set in boldface $(R)$, and matrices are represented by capital letters in sans-serif font $(R)$. Unless otherwise stated, the following symbols denote:

$\begin{array}{ll}\stackrel{d}{=} & \text { Equality in distribution } \\ \mathrm{A}^{*} & \text { Complex conjugate } \\ \mathrm{A}^{T} & \text { Matrix transpose } \\ \mathrm{A}^{\dagger} & \text { Complex conjugate transpose } \\ \operatorname{Tr}(\mathrm{A}) & \text { Trace operator } \\ \left\{\lambda_{i}: \mathrm{A}\right\} & \text { Eigenvalues of A } \\ |\mathrm{A}| & \text { Matrix determinant } \\ \|\mathrm{B}\| & \text { Vector norm } \\ \mathrm{I} & \text { Identity matrix } \\ E[\cdot] & \text { The expectation of a random quantity } \\ \operatorname{Re}[\cdot] & \text { The real part of a complex quantity } \\ \operatorname{Pr}(\cdot) & \text { Probability of the bracketed event } \\ \operatorname{diag}(\ldots) & \text { Diagonal matrix with elements ... on main diagonal }\end{array}$

Throughout this thesis, explanations for variables immediately follow their first introduction in the text or in an equation. 


\section{Contents}

1 Introduction 16

1.1 Motivation ........................ 16

1.2 Thesis Outline . . . . . . . . . . . . . . . . . . . . 19

2 A Review of Matched-Field Parameter Estimation and Performance $\begin{array}{ll}\text { Analysis } & 21\end{array}$

2.1 Matched-Field Parameter Estimation . . . . . . . . . . . . . . 21

2.1.1 Normal Modes Representation of the Signal Field . . . . . . 22

2.1.2 Geoacoustic Modeling and Field Sensitivity to Geoacoustic Parameters . . . . . . . . . . . . . . . . 24

2.1.3 Ambiguity Function in Array Processing . . . . . . . . . 25

2.2 Performance Bounds in Parameter Estimation . . . . . . . . . 26

2.2.1 Covariance Inequality for Unknown Deterministic Parameters 28

2.3 Performance Bounds in the Matched-Field Problem . . . . . . . . . 31

2.3.1 Cramer-Rao Bound . . . . . . . . . . . . . . . 33

2.3.2 Barankin Bound .................. 34

2.4 Summary . . . . . . . . . . . . . . . . . . . 36

3 Bayesian Bounds in Matched-Field Parameter Estimation 37

3.1 Bayesian Performance Bounds . . . . . . . . . . . . . . 37

3.1.1 Bayesian Cramer-Rao Bound . . . . . . . . . . . 39

3.1 .2 Weiss-Weinstein Bound . . . . . . . . . . . . . . 41

3.1.3 Ziv-Zakai Bound .................. 43 
3.2 Applications to Matched-Field Parameter Estimation . . . . . . . . 46

3.2.1 Data Model . . . . . . . . . . . . . . . . . 46

3.2.2 Bayesian Cramer-Rao Bound . . . . . . . . . . . . . 48

3.2 .3 Weiss-Weinstein Bound . . . . . . . . . . . . . . . . . 49

3.2.4 Ziv-Zakai Bound .................. . . 51

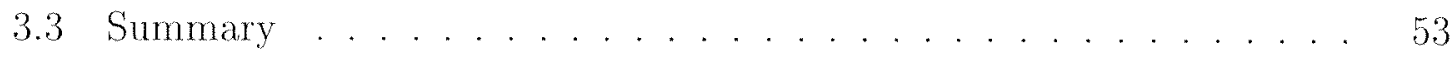

4 Ambiguity Analysis $\quad 54$

4.1 Fundamentals of Ambiguity in the Matched-Field Problem . . . . . . 55

4.1.1 Range-dependence ................ 57

4.1 .2 Depth-dependence ................... 60

4.1.3 Environmental-dependence ............... 61

4.2 Ambiguity Mainlobe: Parameter Coupling . . . . . . . . . . . 63

4.3 Performance Bound and Ambiguity ... . . . . . . . . . 68

4.3.1 Bayesian Cramer-Rao Bound ............. 69

4.3.2 Weiss-Weinstein Bound . . . . . . . . . . . . . 70

4.3.3 Ziv-Zakai Bound ................ 72

4.4 Maximum Likelihood Error Analysis Based on Two-Point Parameter

Estimation . . . . . . . . . . . . . . . . 75

4.4.1 Estimation Errors due to Different Ambiguity Points . . . . 78

4.4 .2 Local Performance Approximation . . . . . . . . . . . . . 83

4.5 Threshold Signal-to-Noise Ratio . . . . . . . . . . . . . . . 86

4.5.1 Estimation of Threshold SNR Based on the Two-Point Estimation Error Analysis . . . . . . . . . . . . . . . . . . 88

4.6 Summary . . . . . . . . . . . . . . . . . . . 90

5 Performance Analysis with Environmental/System Mismatch 92

5.1 Modified Ziv-Zakai Bound . . . . . . . . . . . . . . . . 93

5.2 Application to the Matched-Field Problem . . . . . . . . . . . 96

5.2.1 Mismatched Error Probability . . . . . . . . . . . . . 98

5.2 .2 Mismatch Analysis . . . . . . . . . . . . . . . 101 
5.3 Example: Mismatch Analysis in Bearing Estimation . . . . . . . . 105

5.4 Summary . . . . . . . . . . . . . . . . . 112

6 Examples $\quad 113$

6.1 Computational Issues . . . . . . . . . . . . . . . . . . . . 114

6.1.1 Bayesian Cramer-Rao Bound . . . . . . . . . . . . 114

6.1.2 Weiss-Weinstein Bound . . . . . . . . . . . . 115

6.1.3 Ziv-Zakai Bound .................. 116

6.1 .4 Computational Load . . . . . . . . . . . . . 117

6.2 Source Range Estimation with Perfectly Known Environment . . . 119

6.2 .1 Number of Snapshots . . . . . . . . . . . . . . . . 123

6.2 .2 Incoherent Frequency Averaging . . . . . . . . . . . 126

6.3 Environmental Parameter Estimation . . . . . . . . . . . . . . . 132

6.3.1 Gaussian Parameter Model . . . . . . . . . . . . . . . 136

6.4 Source Range Estimation with Unknown Depth . . . . . . . . . 138

6.4.1 Comments on Numerical Computation . . . . . . . . . . 141

6.5 Source Range Estimation with Environmental Uncertainty . . . . . 143

6.6 Source Range Estimation with Environmental Mismatch . . . . . . 152

6.7 Comparison to SWellEX-3 Data Processing . . . . . . . . . . . 157

6.8 Summary and Discussions . . . . . . . . . . . . . 165

7 Concluding Remarks $\quad 168$

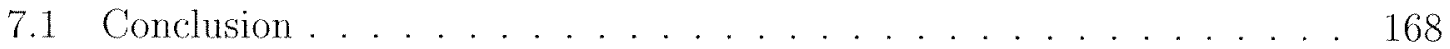

7.2 Summary of Contributions . . . . . . . . . . . . . . 169

7.3 Future Work . . . . . . . . . . . . . . . . . 170

A Weiss-Weinstein Bound Derivations for MFP/MFT 172

A.1 Derivations of $\mu(s, \mathbf{h}) \ldots \ldots \ldots \ldots 172$

A.2 Derivations of $\eta\left(f_{m}, \boldsymbol{\theta}, \mathbf{h}\right) \ldots \ldots \ldots \ldots . \ldots \ldots 74$

A.3 Some Properties of $\eta\left(f_{m}, \boldsymbol{\theta}, \mathbf{h}\right) \ldots \ldots \ldots \ldots . \ldots \ldots$

A.3.1 Proof of $\eta\left(f_{m}, \boldsymbol{\theta}, \mathbf{h}\right) \geq 1$ and Conditions for Equality . . . . 178 


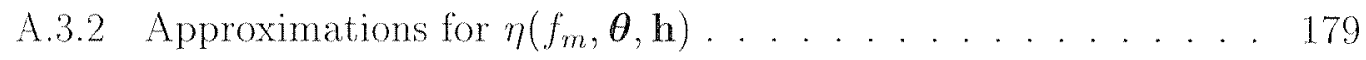

A.3.3 A Similar Result for the Minimum Variance Distortionless Pro-

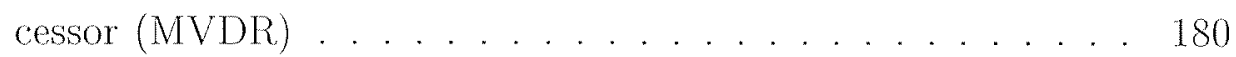

B Maximum Likelihood Estimator of Source/Environmental Parameters

C Error Analysis of Random Signal Parameter Estimation through Binary Hypothesis Test 183

C.1 Case 1: Single-Frequency Component . . . . . . . . . . . . 185

C.1.1 Lindsey's Approach . . . . . . . . . . . . . . . . . . . 185

C.1.2 Richmond's Approach . . . . . . . . . . . . . 192

C.2 Case 2: Multiple-Frequency Component . . . . . . . . . . 196

C.2.1 Exact Error Probability . . . . . . . . . . . . . 199

C.2.2 Approximation to Error Probability . . . . . . . . . . . 201

D Issues on Signal-to-Noise Ratio 204 


\section{List of Figures}

1-1 Example signal field correlation . . . . . . . . . . . . 17

$1-2$ Example MLE ambiguity surface . . . . . . . . . . . 18

2-1 Model for the seismo/acoustic environment . . . . . . . . . . . . . 22

$2-2$ General parameter estimation model . . . . . . . . . . 27

3-1 Valley filling function . . . . . . . . . . . . . . . . . . 44

4-1 An example shallow water environment . . . . . . . . . . 55

4-2 Signal ambiguity function for source range estimation . . . . . . 58

4-3 Signal ambiguity function using full column spanning array . . . . . 58

4-4. Signal ambiguity function for source depth estimation . . . . . . . . 61

4-5 Signal ambiguity function for sediment wave-speed estimation . . . . 62

4-6 Signal ambiguity function for sediment thickness estimation . . . . 63

4-7 Ambiguity mainlobe contour for source range and depth estimation . 65

4-8 Signal ambiguity function for sediment top and bottom wave-speed estimation ......................... 66

4-9 Signal ambiguity function for source range and sediment thickness estimation ......................... 67

4-10 Signal ambiguity function for source range and sediment wave-speed

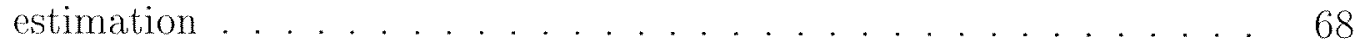

4-11 Signal ambiguity function for source range estimation with/without depth uncertainty ........................ 75 
4-12 Signal ambiguity function for source range estimation with/without sediment bottom wave-speed uncertainty . . . . . . . . . 76

4-13 Two-point estimation error probability . . . . . . . . . . 78

4-14 Probabilistic square error at each scanning source range . . . . . . . 80

4-15 Probabilistic square errors at different sidelobe peak points . . . . . . 81

4-16 Probabilistic square errors at different sidelobe points around a sidelobe peak ............................. 81

4-17 Probabilistic square errors at different mainlobe points . . . . . . . 82

4-18 Probabilistic square errors at different ambiguity points . . . . . . . 83

4-19 Division of the parameter interval for definition of the M-ary detection

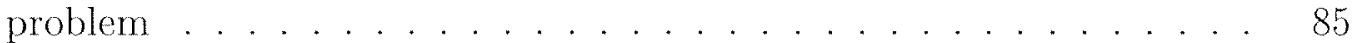

4-20 Local mean square error for source range estimation . . . . . . . . . . 86

4-21 Typical larger error performance bound . . . . . . . . . . . . . . . 87

5-1 Mismatched ambiguity function for source range estimation . . . . . . 104

5-2 Mismatched error probability for source range estimation . . . . . . . 104

$5-3$ Uniform linear array . . . . . . . . . . . . . . . . . 106

5-4 Ambiguity function for bearing estimation . . . . . . . . . . 107

5-5 Bearing estimation mean square error . . . . . . . . . . . . . 109

5-6 Probabilistic square error for bearing estimation . . . . . . . . . . . 109

5-7 Mismatched ambiguity function for bearing estimation . . . . . . . 110

5-8 Bearing estimation mean square error with slight mismatch . . . . . . 111

5-9 Bearing estimation mean square error with moderate mismatch . . . 112

6-1 Performance bound evaluation for source range estimation with perfectly known environment . . . . . . . . . . . . . 120

6-2 Local Fisher information for source range estimation . . . . . . 120

6-3 Example signal ambiguity function for source range estimation . . . . 122

6-4 Performance bound as a function of the snapshot number . . . . . . . 124

6-5 Probabilistic square error as a function of the snapshot number . . . 125

6-6 Performance bound evaluation with a single snapshot . . . . . . . 126 
6-7 Probabilistic square errors at different ambiguity points: single snapshot case . . . . . . . . . . . . . . . . 127

6-8 Performance bound evaluation with ten frequency components and ten snapshots for each frequency . . . . . . . . . . . . . . . 128

6-9 Performance bound evaluation with ten frequency components and single snapshot for each frequency . . . . . . . . . . . . . . 129

6-10 Ziv-Zakai bound with single versus ten frequency components . . . . 129

6-11 Example signal ambiguity functions at different frequencies . . . . . 130

6-12 Probabilistic square errors at different frequencies . . . . . . . . . . 131

6-13 Probabilistic square error: single frequency versus ten frequencies . . 131

6-14 Performance bound evaluation for sediment top wave-speed estimation 133

6-15 Performance bound evaluation for sediment bottom wave-speed esti-

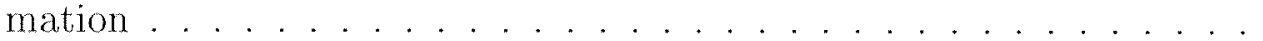

6-16 Performance bound evaluation for sediment thickness estimation with uniform prior distribution . . . . . . . . . . . . . 135

6-17 Probabilistic square errors for sediment thickness at different ambiguity

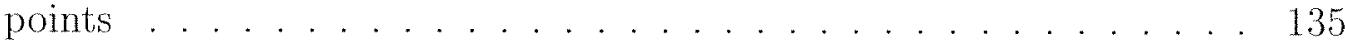

6-18 Performance bound evaluation for sediment thickness estimation with Gaussian prior distribution . . . . . . . . . . . . . 138

6-19 Performance bound evaluation for source range estimation with depth uncertainty . . . . . . . . . . . . . . . . . 140

6-20 Error coupling between source range and depth . . . . . . . . . 140

6-21 Probabilistic square errors at different range-depth projected ambiguity points .......................... 141

6-22 Contour of threshold SNR predicted at individual source locations . . 142

6-23 Histogram of threshold SNR at individual source locations . . . . . . 142

6-24 Averaged error probability in evaluation of the ZZB: range and depth 144

6-25 Performance bound evaluation for source range estimation with environmental uncertainty . . . . . . . . . . . . . 145 
6-26 Ziv-Zakai bound evaluation for source range estimation with/without environmental uncertainty . . . . . . . . . . . . . . 146

6-27 Signal ambiguity function for source range estimation with/without known sediment wave-speeds . . . . . . . . . . . . . . . 147

6-28 Error coupling between source range and sediment wave-speeds . . . . 147

6-29 Probabilistic square error at each scanning source range with unknown sediment wave-speeds .................... 149

6-30 Contour of threshold SNR predicted at individual range-wave-speed

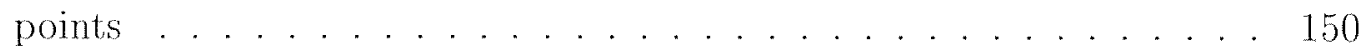

6-31 Histogram of threshold SNR predicted at individual range-wave-speed points: sidelobe issue . . . . . . . . . . . . . . . . 150

6-32 Histogram of threshold SNR predicted at individual range-wave-speed points: mainlobe issue ................ . . 151

6-33 Averaged error probability in evaluation of the ZZB: range and wave-

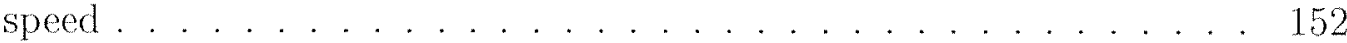

6-34 Performance bound evaluation for source range estimation with environmental mismatch . . . . . . . . . . . . . 153

6-35 Probabilistic square error with mismatched environment . . . . . . 154

6-36 Probabilistic square errors at different ambiguity points: mismatched

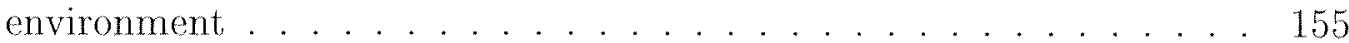

$6-37$ Bias in source range estimation . . . . . . . . . . . . . . 155

6-38 Performance bound evaluation at different true sediment top wave-speeds156

6-39 Contour of bias in source range estimation . . . . . . . . . . 157

6-40 Performance bound evaluation for source range estimation with different environmental mismatches . . . . . . . . . . . . 158

6-41 SWellEX-3 experiment area ................. 160

6-42 Optimized SWellEX-3 environment . . . . . . . . . 160

6-43 SWellEX-3 source localization results at SNR $=10 \mathrm{~dB} \ldots \ldots 1$

6-44 SWellEX-3 source localization results at SNR $=0 \mathrm{~dB} \ldots \ldots 161$

6-45 SWellEX-3 source localization results at SNR $=-4 \mathrm{~dB} \ldots \ldots . . . .162$ 
6-46 SWellEX-3 source localization results at SNR $=-8 \mathrm{~dB} \ldots \ldots 162$

6-47 SWellEX-3 source range estimation at SNR $=10$ and $0 \mathrm{~dB} \ldots 163$

6-48 SWellEX-3 source range estimation at SNR $=-4$ and $-8 \mathrm{~dB} \ldots \ldots 4$

6-49 Ziv-Zakai bound evaluation for source range estimation . . . . . . . 164

6-50 Histogram of threshold SNR at individual source/array locations . . 165

6-51 SWellEX-3 source localization ambiguity surface . . . . . . . . . 166

6-52 SWellEX-3 source range estimation: DGPS versus MLE . . . . . . . 166 


\section{List of Tables}

4.1 Threshold SNR predictions at individual true parameter points in source range estimation . . . . . . . . . . . . . . . . . 90

4.2 Threshold SNR predictions at individual true parameter points with a 26-sensor column spanning array . . . . . . . . . . . . . . . 91

4.3 Threshold SNR predictions at individual true parameter points with a 16-sensor column spanning array . . . . . . . . . . . . . . . 91

6.1 Computational load in evaluation of performance bound . . . . . 118

6.2 Threshold SNR prediction in sediment thickness estimation . . . . . . 136

6.3 True and assumed environmental models . . . . . . . . . . . . . . 158 


\section{Chapter 1}

\section{Introduction}

\subsection{Motivation}

Matched-field estimation is a parameter estimation technique that works by matching the measured signal field with the modeled signal field. The well-known matchedfield processing (MFP) and matched-field tomography (MFT) can both be fit into this class of parameter estimator [8]. They concern estimation of source location and/or ocean environmental model parameters; for each hypothesized parameter set, acoustic propagation through the channel is predicted; correlation between the observed signal and the predicted signal defines an ambiguity surface, and an estimate of the parameter set is derived from the highest peak.

Matched-field methods have been developed for about twenty-five years [8]. The major theoretical and experimental developments in the past concentrate on the high signal-to-noise ratio (SNR) region [6]. However, one often has to deal with low SNR scenarios, for example, in passive source localization. For those scenarios, matchedfield performance has not been well investigated.

Figs. 1-1 and 1-2 present some examples of source localization under a given shallow water environment (Fig. 4-1) using the maximum likelihood estimator (MLE) [43, see also Appendix B]. In the absence of noise (Fig. 1-1), the ambiguity surface is the

signal field correlation associated with the true source position and each scanning source position. Since the typical field is a highly nonlinear function of the embedded 


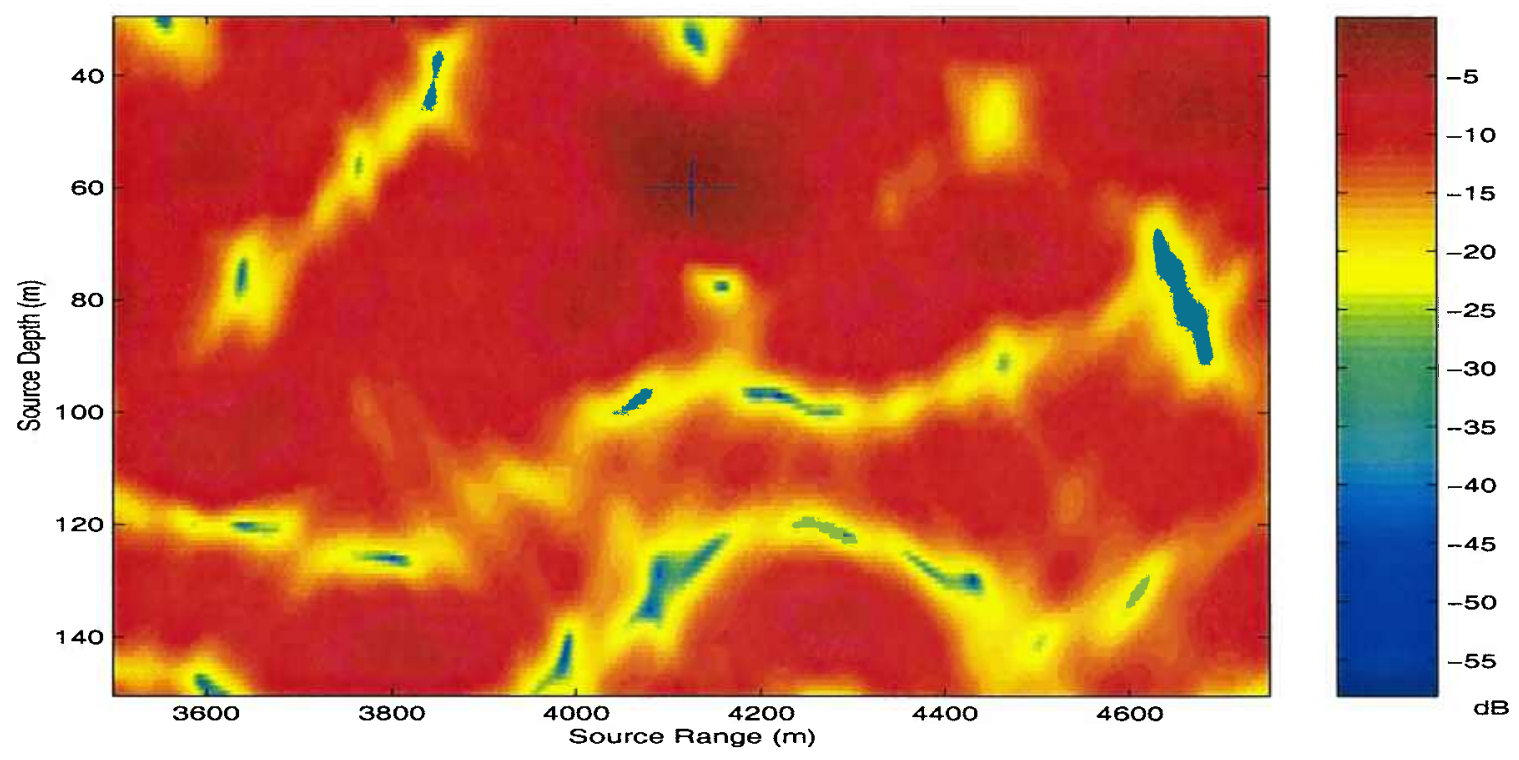

Figure 1-1: Example signal field correlation. '+' indicates the true source position.

parameters, the ambiguity surface is often characterized by a multi-modal structure. In addition to the mainlobe around the true parameter, there are many sidelobes. At high SNR, most ambiguity outputs have the maximum at mainlobe points (Fig. 12(a)). However, as SNR decreases, more ambiguity peaks occur at sidelobe points (Fig. 1-2(b)); below a threshold SNR, the estimation mean square error (MSE) is dominated by sidelobe errors.

This threshold phenomenon is often understood in terms of the parameter estimation theory bounds. For sufficiently high SNR or long observation time, the MLE performance is predicted by the Cramer-Rao lower bound (CRB) [43]. The CRB bounds the variance of small errors around the true parameter and has been intensively used for the matched-field problem $[9,54,10,47]$. However, for low SNR and short observation time, the CRB is no longer achievable by the MLE due to the sidelobe effect, and a large-error bound is necessary to specify the attainable performance. For example, the Barankin bound $[11,66]$ is one of the prevalent techniques for this purpose.

As a local performance bound, the Barankin bound does not exploit any a priori parameter information, and is limited to unbiased estimates. An MLE with nonlinear parameter-dependence is often biased in the low SNR region; in this case, even a large- 

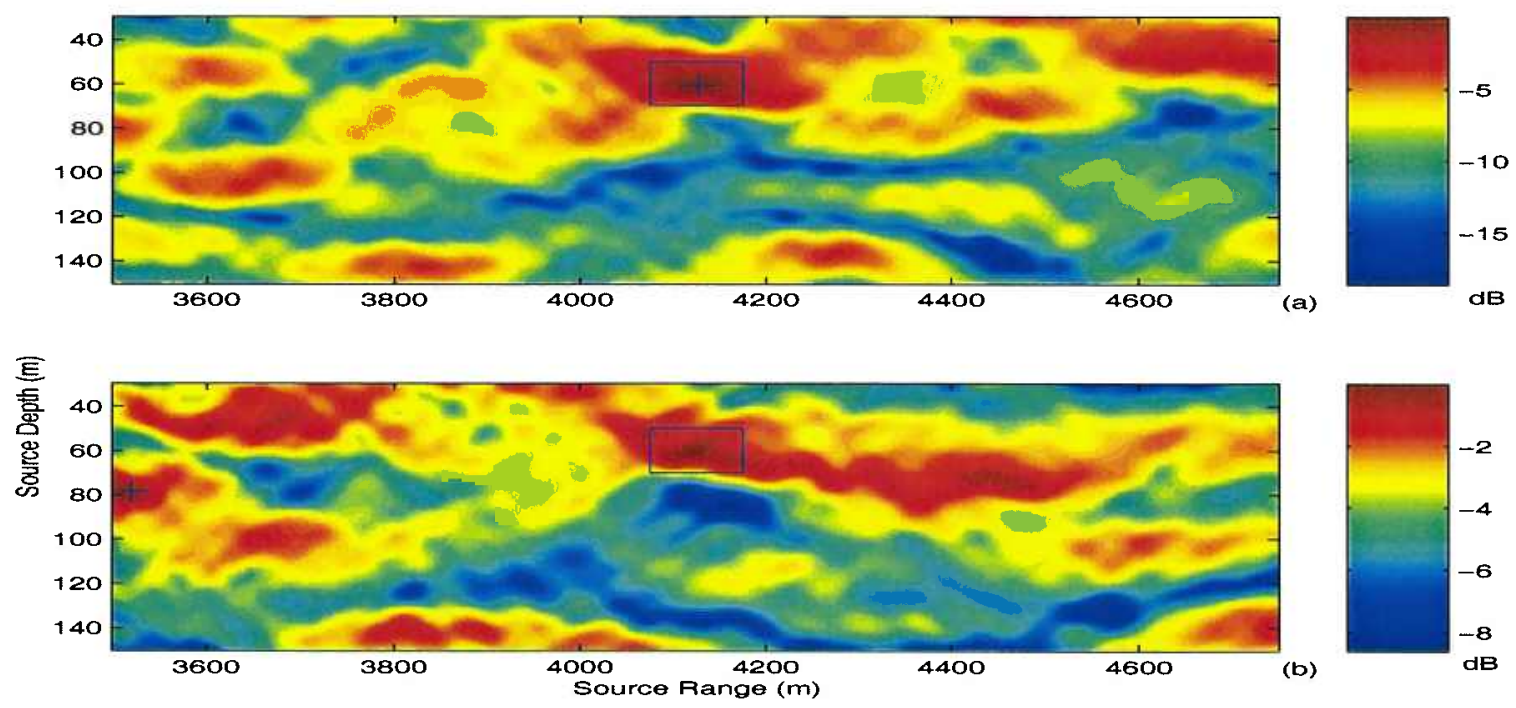

Figure 1-2: Example MLE ambiguity surface for input SNR = (a) $0 \mathrm{~dB}$; (b) $-8 \mathrm{~dB}$. ' + ' indicates the peak output position; the rectangular box specifies the mainlobe region.

error local bound can still be far less tight. In contrast, some Bayesian (global) bounds including the Ziv-Zakai bound (ZZB) [84] and Weiss-Weinstein bound (WWB) [79] assume a random parameter model with known a priori distribution, thus free from the bias assumption. They are tight and reliable at all SNR regions with the cost of additional computational and analytical complexity.

In this thesis, we formulate the Weiss-Weinstein bound and Ziv-Zakai bound for the matched-field estimation problem. In addition to some performance advantages mentioned, a Bayesian bound naturally incorporates environmental uncertainty into the source localization problem by assuming a random parameter model for each uncertain environmental parameter. The major drawback is the increased complexity as the number of unknown parameters increases. Nonetheless, for most problems of practical interest, effects of the environmental uncertainty are often dominated by only a few parameters, so the global performance bounds can be well applied.

The threshold phenomenon has been investigated in the context of traditional array processing for time-delay or bearing estimation, where the ambiguity output is often quasi-periodic and independent of the specific parameter value $[80,77]$, and 
the global, large-error bounds have been successfully applied $[80,78,14]$. Matchedfield methods achieve performance improvement over the traditional array processing by using a full field signal representation instead of the plane wave assumption. In this way, the physics of the signal (and/or noise) structure is incorporated into the parameter estimation problem but the resulting ambiguity structure is often more complicated. Signal ambiguity function is a measure of the invertibility of the signal field. Therefore, issues on the output mainlobe width and sidelobe levels are very important for the development of any matched-field algorithm, which are investigated in this thesis in the context of the MLE.

The full field signal solution, however, requires an accurate environmental model, which is often unavailable. In many practical applications, an environmental model is assumed per one's best knowledge on the test site. It is very common that this assumed model could differ from the true one, leading to seriously biased estimates even at high SNR. This is the so-called mismatch problem [8]. There are considerable efforts toward analyzing this mismatch problem, but a quantitative link between the size of mismatch and the mean square error in parameter estimation has not been established yet. Developments in this thesis lead to such a performance analysis method for any replica-correlation-based estimation problem with environmental/system mismatch.

\subsection{Thesis Outline}

The thesis is organized as follows.

Chapter 2 first gives a brief introduction to some involved areas including sound waveguide propagation, matched-field parameter estimation and performance bound in parameter estimation. The existing research on matched-field performance analysis is then reviewed.

Chapter 3 applies the Bayesian approach to matched-field parameter estimation. A general framework for Bayesian parameter estimation is first defined. Under this framework, three Bayesian bounds, Bayesian Cramer-Rao bound, Weiss-Weinstein 
bound and Ziv-Zakai bound, are reviewed. These bounds are then developed using a general random signal model typical of the matched-field problem.

Chapter 4 investigates some ambiguity issues in the matched-field problem. These include the origin of ambiguities in terms of the normal modes representation and the mainlobe ambiguity in terms of parameter coupling. Behavior of each performance bound is also discussed in relation to the ambiguities. To analyze the effects of different ambiguity points, either mainlobe or sidelobe points, some approximations based on the two-point estimation error probability are exploited. These results are then used to find the threshold SNR in matched-field parameter estimation.

Chapter 5 proposes a modified Ziv-Zakai bound to incorporate environmental/ system mismatch. The mismatch analysis is then developed in the context of the matched-field problem, and demonstrated using a bearing estimation example, in which the array tilt angle is mismatched.

Chapter 6 first discusses some computational issues that arise in evaluation of each bound in relation to the field sensitivity and ambiguity. The theoretical bounds are then tested to the MLE simulations of source localization and environmental parameter inversion for a typical shallow-water propagation environment. Effects of environmental uncertainties and mismatch on source localization are investigated as well. Finally, the theoretical predictions are compared to the real data processing.

Chapter 7 summarizes the thesis and proposes issues for future efforts.

Appendix A gives the derivations of the Weiss-Weinstein bound in the matchedfield problem.

Appendix B derives the matched-field maximum likelihood estimate for source localization and estimation of environmental parameters.

Appendix $\mathrm{C}$ addresses the two-point estimation problem. The related error probability is derived using both narrowband and broadband (incoherent multi-tone) data models. These results play a significant role for evaluation of the Ziv-Zakai bound (both standard and modified) as well as ambiguity analysis.

Appendix D defines the signal-to-noise ratio used in this thesis. Note that unless otherwise stated, we use input, sensor-averaged SNR through the entire thesis. 


\section{Chapter 2}

\section{A Review of Matched-Field Parameter Estimation and Performance Analysis}

\subsection{Matched-Field Parameter Estimation}

Matched-field processing (MFP) has been intensively investigated in ocean acoustics $[8,39]$, aiming at accurately and remotely localizing underwater targets. It is a generalization of the traditional plane wave beamforming in that the plane wave beamforming matches the measured field with the plane wave for each look direction, while MFP matches the measured field with the full field solution to the sound waveguide propagation for each scanning source position. Therefore, when the environmental model is accurate, a significant performance improvement by MFP is expected. On the other hand, because MFP source localization requires precise environmental information, any mismatch between the assumed environmental parameter values and the actual values could degrade the performance seriously. Thus, matched-field tomography (MFT) is concerned with estimation of environmental parameters [71]. Similar to MFP, MFT exploits the interference pattern generated between array sensors but reverses the roles of source position and environmental information. 


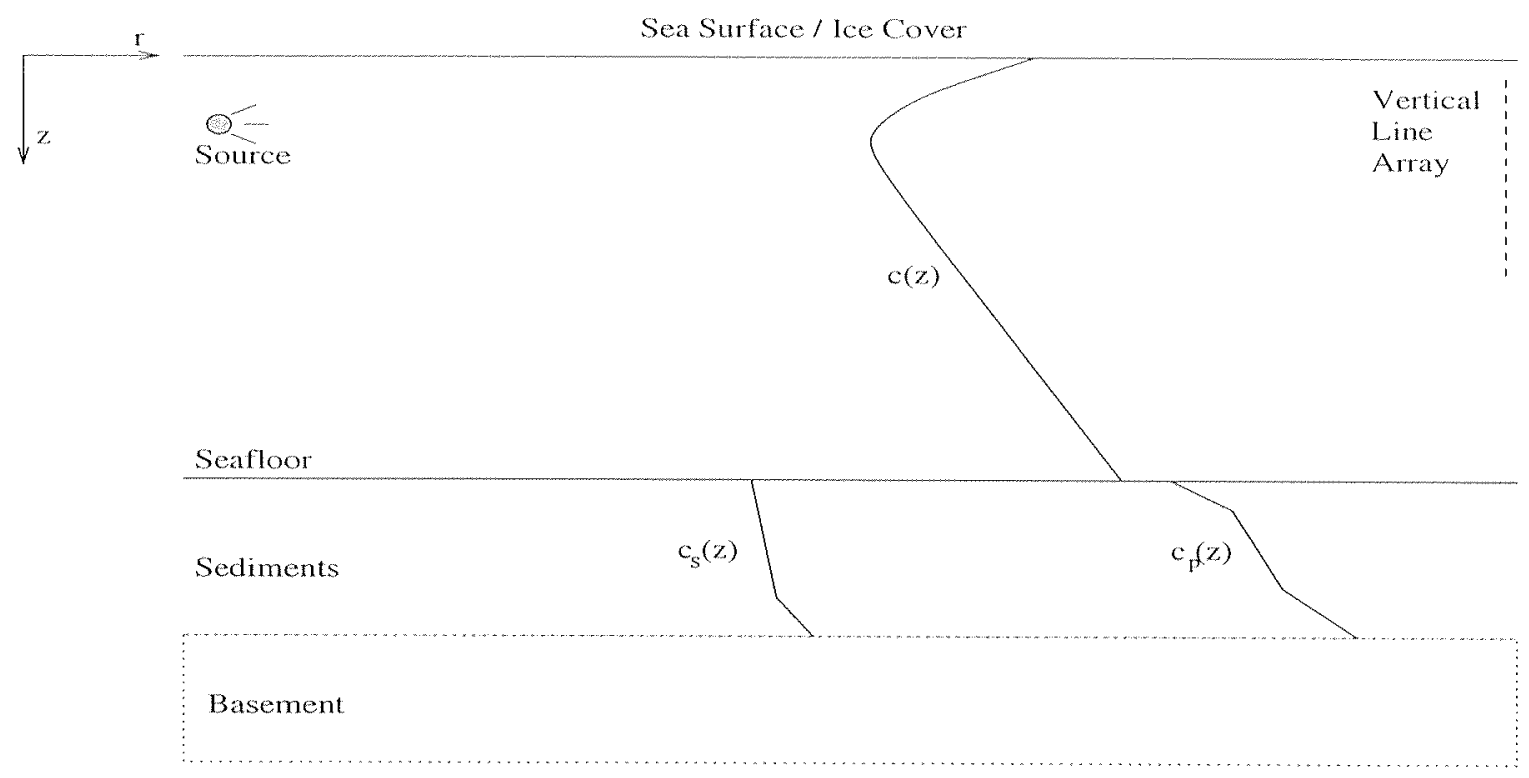

Figure 2-1: Model for the seismo/acoustic environment.

Developments of matched-field methods are closely related to developments in physical modeling of acoustic waveguide propagation, numerical simulation of the acoustic field, and array signal processing techniques. Some of the issues relevant are discussed in the following sections.

\subsubsection{Normal Modes Representation of the Signal Field}

Consider a stratified waveguide model for the seismo/acoustic environment shown in Fig. 2-1, which consists of water column, multi-layer sediment and basement, and can be either range-independent (as plotted) or dependent. A point source radiates narrowband or broadband signal and the acoustic field is sampled by a vertical receiver array. The modeled acoustic field is often taken from the solution, exact or approximated, of the wave equation governing the sound propagation in a given environment.

Let $\mathbf{r}=(r, z)$ denote the position coordinates, where $r$ is the range and $z$ is the depth. Consider a point source at $\mathbf{r}_{0}=\left(0, z_{0}\right)$. Given the sound velocity profile $c(z)$ (in the sediment layer this is $c_{p}(z)$ for compressional wave-speed, or $c_{s}(z)$ for shear wave-speed) and the density stratification $\rho(z)$, the frequency-domain wave equation 
for the resulting pressure field is [32]:

$$
\rho(z) \nabla \cdot\left[\frac{1}{\rho(z)} \nabla p(\mathbf{r})\right]+k^{2}(z) p(\mathbf{r})=-2 \frac{\delta(r)}{r} \delta\left(z-z_{0}\right)
$$

where $k(z)=\frac{2 \pi f}{c(z)}$ is the wavenumber, and $f$ is the frequency.

Coupled with the wave equation are the boundary conditions between different medium layers, such as the air-sea interface and the water-sediment interface. Regardless of the simple form of the wave equation, there is no exact solution for the wave field, except for some extremely simple cases. Various approximations have been developed, for example, the wavenumber integration model, the normal modes model, and their coupled versions for range-dependent environment [42]. Among them, the method of normal modes is often used because its simple implementation and good physical interpretation. Using the normal modes decomposition, the pressure in the far field has the form

$$
p(r, z) \approx \frac{\sqrt{2 \pi} e^{i \pi / 4}}{\rho\left(z_{0}\right)} \sum_{n=1}^{n_{\max }} u_{n}^{*}\left(z_{0}\right) u_{n}(z) \frac{e^{i k_{n} r}}{\sqrt{k_{n} r}}
$$

where $u_{n}(z)$ denotes the modal depth eigenfunction for mode $n$, and $k_{n}$ denotes the corresponding horizontal wavenumber. The wave attenuation is often included as the imaginary part of the wavenumber. $n_{\max }$ is the number of propagation modes, which is determined by system/environmental parameters. Some leaky modes, which have deeper bottom penetrations, can also be included, but their contributions are insignificant at long-range due to large attenuation.

Solution to both the unit point source wave equation in (2.1) and the boundary conditions defines the Green's function, which is the transfer function of an ocean propagation system. Obviously, it is a function of source-receiver configuration as well as environmental information. The receiver output can then be expressed as a product of the input source term and the Green's function.

A number of codes have been developed to simulate the acoustic field for a given environmental configuration. Typical normal modes codes include KRAKEN [56] and 
SuperSnap [41]. Others include OASES wavenumber integration codes [62], and RAM parabolic equation codes [25]. Despite their strengths in different special scenarios, they often give consistent field replicas for general propagation problems.

\subsubsection{Geoacoustic Modeling and Field Sensitivity to Geoa- coustic Parameters}

According to Hamilton [36], a geoacoustic model is defined as a model of the real sea floor with emphasis on measured, extrapolated, and predicted values of those properties important in underwater acoustics and those aspects of geophysics related to sound transmission. A complete model includes water-mass data, a bathymetric chart, and the thickness and material properties for each sediment or rock layer. The typical material properties include compressional wave-speed, shear wave-speed, compressional wave attenuation, shear wave attenuation, and density. In general, these properties are depth-dependent and frequency-dependent. Moreover, they may vary significantly over geographical position, so it requires tremendous efforts to detail

the geoacoustic model for even a small ocean area. As a result, these properties are usually approximated, thus limiting the modeling accuracy for acoustic propagation in the ocean.

Geoacoustic parameters enter the sound propagation via strong signal boundary interactions. Therefore, the reflectivity property at the boundary plays an important role to couple the bottom effects to the sound propagation. A particular case of interest is the reflection from a high velocity half-space, where a total internal reflection takes place. The velocity contrast above and below the boundary determines a critical angle of reflection [20]. For the lossless case, when the incident angle exceeds the critical angle, the magnitude of the reflection coefficient is exactly one, and the bottom property dependence is in the phase term. This phenomenon leads to the trapped modes representation of the field. When the incident angle is less than the critical angle, the phase term is zero and the bottom property dependence is in the attenuated magnitude term, which leads to the leaky modes representation. 
However, for the lossy bottom, both the trapped and leaky modes are affected by the bottom attenuation, and the total reflection no longer exists. The above results suggest that the presence and the value of the sound velocity gradient are of considerable importance in the sound propagation and its dependence on the bottom environment.

Capability of the matched-field tomography to estimate the bottom geoacoustic parameters is from the field sensitivity to these parameters, and this field sensitivity issue has long been an important research topic aiming to compare the influence of each parameter on the field with respect to depth, range and frequency. In a specific environment, this leads to ordering the parameters from the most sensitive to the least sensitive, and this ordering basically determines the relative performance in estimation of each individual parameter.

Seen from the normal modes field representation, the term of $\exp \left(i k_{n} r\right)^{1}$ may play a key role in the sensitivity issue. A small perturbation of the horizontal wavenumber due to a small parameter perturbation is amplified by range, particularly at longrange. In Ref. [45], the field sensitivity is considered as the combined effects of (1) a uniform change of the long-range phase common to all modes; (2) a nonuniform (jumbling) of the relative phase between modes; and (3) changes in energy loss. If the first factor dominates, a narrowband wavenumber spectrum assumption is quite reasonable. However, if the second factor dominates, we have to deal with an equivalent spatial wideband process.

It is worth noting that although a strong field sensitivity increases the estimation performance, it makes the estimation subject to environmental mismatch.

\subsubsection{Ambiguity Function in Array Processing}

Various matched-field processing algorithms have been developed, most of which are based on traditional array processing techniques in radar/sonar applications. A matched-field algorithm estimates the field distribution versus a possible location/ environmental parameter set, and usually produces an output in terms of the am-

\footnotetext{
${ }^{1}$ An equivalent time-delay expression is $\exp \left(j 2 \pi f_{0} \cdot \tau_{n}(r)\right)$, where $f_{0}$ is the carrier frequency and $\tau_{n}(r)$ is the propagation delay for mode $n$.
} 
biguity function. An estimate of the unknown parameter set is then obtained by locating the maximum output (best signal match).

Let $\mathrm{G}(f, \boldsymbol{\theta})$ be the Green's function across the receiver array associated with a given set of source/environmental parameters, $\theta$. For two sets of specific parameter values, $\boldsymbol{\theta}_{1}$ and $\boldsymbol{\theta}_{2}$, a simple definition of the ambiguity function is the normalized field correlation [8], i.e.,

$$
\Gamma\left(f, \boldsymbol{\theta}_{1}, \boldsymbol{\theta}_{2}\right)=\left|\left(\frac{\mathrm{G}\left(f, \boldsymbol{\theta}_{1}\right)}{\left\|\mathrm{G}\left(f, \boldsymbol{\theta}_{1}\right)\right\|}\right)^{\dagger}\left(\frac{\mathrm{G}\left(f, \boldsymbol{\theta}_{2}\right)}{\left\|\mathrm{G}\left(f, \boldsymbol{\theta}_{2}\right)\right\|}\right)\right|^{2} .
$$

Despite its simple form, most matched-field outputs can be expressed in terms of this fundamental component.

The MFP/MFT ambiguity function often demonstrates some complicated multimodal structures, particularly when the dimensionality of the parameter space is high. Some global optimization algorithms such as simulated annealing [26] and genetic algorithm [33] have been developed in searching the multi-dimensional parameter space. Currently, performance analyses on these ad hoc methods are often based on Monte Carlo simulations.

\subsection{Performance Bounds in Parameter Estimation}

Parameter estimation performance bounds have been studied in the information theory and signal processing literature since the 1940s. To review the existing bounds, let us define a general model for any parameter estimation problem, as shown in Fig. 2-2 [72]. The model consists of four components defined by

Parameter space consisting of deterministic or random parameters, $\theta$;

Observation space consisting of observation vectors, $\mathbf{R}$;

Probabilistic mapping from parameter space to observation space governing the effect of $\theta$ on observations, which can be described by the probability density function of the observation conditioned on $\boldsymbol{\theta}, p(\mathbb{R} ; \boldsymbol{\theta})$ for non-random 


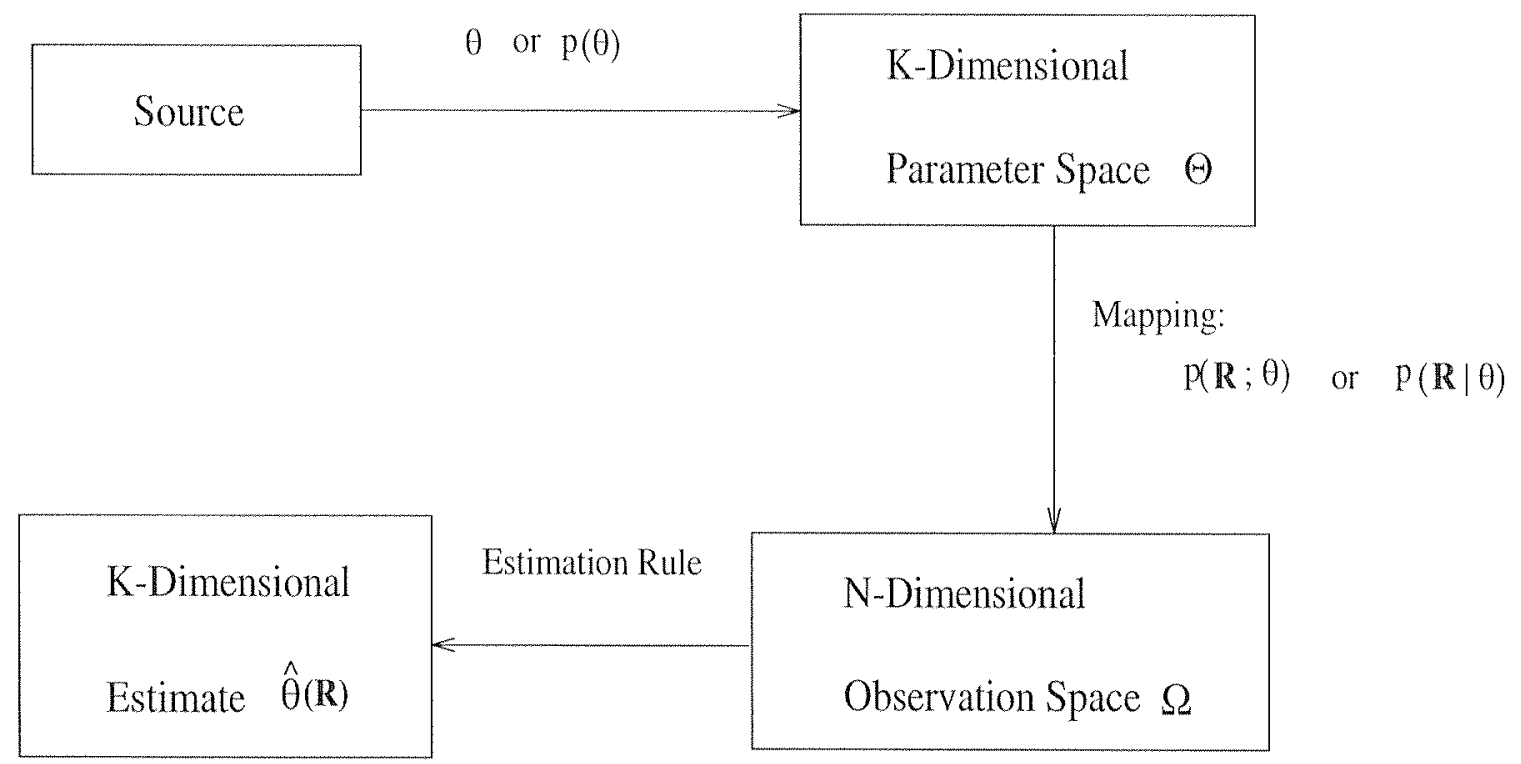

Figure 2-2: General parameter estimation model (from [72]).

parameters, or $p(\mathbf{R} \mid \theta)$ for random parameters; and

Estimation rule mapping the observation space into estimates of the parameters, $\hat{\theta}(\mathbf{R})$.

In general, the estimation rule is chosen according to some criterion, which is stated in terms of the quality measure of the estimation procedure. For the singleparameter case, typical performance measures include bias, variance, and mean square error as defined below: ${ }^{2}$

$\operatorname{Bias} E[\hat{\theta}(\mathbf{R})-\theta]$

Variance $\operatorname{Var}(\hat{\theta}(\mathbf{R}))=E\left[(\hat{\theta}(\mathbf{R})-E[\hat{\theta}(\mathbf{R})])^{2}\right]$,

Mean Square Error (MSE) $E\left[(\hat{\theta}(\mathbf{R})-\theta)^{2}\right]$

There is a simple relationship coupling these three quantities:

$$
\operatorname{Var}(\hat{\theta}(\mathbf{R}))=E\left[(\hat{\theta}(\mathbf{R})-\theta)^{2}\right]-E^{2}[\hat{\theta}(\mathbf{R})-\theta]
$$

${ }^{2}$ For the vector parameter case, one can define the MSE matrix in a similar way as given in Section 2.2.1 and 3.1. 
In the random parameter case, the mean square error is often of interest, while in the non-random parameter case, the bias and variance are more pertinent measures. However, most practical parameter estimation algorithms are developed from ad hoc considerations, and calculations of the performance measures for these algorithms are usually difficult, if not impossible. Rather than approaching the problem directly, people have developed lower bounds on the mean square error to specify the fundamental performance limits regardless of any specific estimation method.

There are two types of performance bounds, local bounds and global bounds, which bound the local and global mean square errors for non-random and random parameter models, respectively. Most of them originate from the corresponding covariance inequality as elaborated in the following.

\subsubsection{Covariance Inequality for Unknown Deterministic Pa- rameters}

Consider a set of unknown, but non-random parameters $\boldsymbol{\theta}$. Let $\mathbf{T}(\mathbf{R})$ be a statistic of the observation with mean $E[\mathbf{T} ; \boldsymbol{\theta}]=\boldsymbol{\theta}+\mathrm{b}(\boldsymbol{\theta})$, where $\mathrm{b}(\boldsymbol{\theta})$ is the bias, and

$$
E[(\cdot) ; \boldsymbol{\theta}]=\int_{\Omega}(\cdot) p(\mathbb{R} ; \boldsymbol{\theta}) d \mathbf{R}
$$

Denote $\Upsilon(\mathbf{R}, \boldsymbol{\theta})$ as an arbitrary matrix-valued function with finite second moment. Then the following covariance inequality $[40,48,2]$ sets up a bound on the MSE of the statistic $\mathrm{T}$ :

$$
\begin{aligned}
& \Sigma(\boldsymbol{\theta})=E\left[(\mathbf{T}-\boldsymbol{\theta})(\mathbf{T}-\boldsymbol{\theta})^{T} ; \boldsymbol{\theta}\right] \geq \mathbf{b}(\boldsymbol{\theta}) \mathbf{b}^{T}(\boldsymbol{\theta}) \\
& +E\left[(\mathbf{T}-E[\mathbf{T} ; \boldsymbol{\theta}]) \Upsilon^{T} ; \boldsymbol{\theta}\right] \cdot\left(E\left[\Upsilon \Upsilon^{T} ; \boldsymbol{\theta}\right]\right)^{-1} \cdot E\left[\Upsilon(\mathbf{T}-E[\mathbf{T} ; \boldsymbol{\theta}])^{T} ; \boldsymbol{\theta}\right]
\end{aligned}
$$

Note that there exists a bias term in the above covariance inequality. Because the precise value of the bias is often unavailable, this type of bound is limited to unbiased estimates ${ }^{3}$. Hence in the sequel, the bias term is ignored.

\footnotetext{
${ }^{3}$ The first order bias of a maximum likelihood estimator has been investigated.in Refs. [53, 69].
} 
A series of bounds can be defined by choosing different $\Upsilon(\mathbf{R}, \theta)[2]$. For example,

1. Cramer-Rao and Bhattacharyya bounds

$$
\Upsilon(\mathbf{R}, \boldsymbol{\theta})=\frac{1}{p(\mathbf{R} ; \boldsymbol{\theta})} \cdot \frac{\partial}{\partial \boldsymbol{\theta}} p(\mathbf{R} ; \boldsymbol{\theta})
$$

The MSE matrix is bounded by

$$
\Sigma(\theta) \geq \mathrm{J}_{D}^{-1}(\theta)
$$

where

$$
\mathrm{J}_{D}(\boldsymbol{\theta})=E\left[\frac{\frac{\partial}{\partial \boldsymbol{\theta}} p(\mathbf{R} ; \boldsymbol{\theta}) \cdot \frac{\partial}{\partial \boldsymbol{\theta}^{T}} p(\mathbf{R} ; \boldsymbol{\theta})}{p^{2}(\mathbf{R} ; \boldsymbol{\theta})} ; \boldsymbol{\theta}\right]
$$

is the Fisher information. This is the well-known Cramer-Rao bound. An extension of (2.7) to high-order derivatives leads to the Bhattacharyya bound [2].

2. Barankin bound

$$
\Upsilon(\mathbf{R}, \boldsymbol{\theta})=\frac{1}{p(\mathbf{R} ; \boldsymbol{\theta})} \cdot\left[\begin{array}{c}
p\left(\mathbf{R} ; \boldsymbol{\theta}_{1}\right)-p(\mathbf{R} ; \boldsymbol{\theta}) \\
\cdots \\
p\left(\mathbf{R} ; \boldsymbol{\theta}_{K}\right)-p(\mathbf{R} ; \boldsymbol{\theta})
\end{array}\right]
$$

The resulting bound is the multi-dimensional Chapman-Robbins bound [21]:

$$
\Sigma(\boldsymbol{\theta}) \geq \sup _{\left\{\theta_{1}, \ldots, \theta_{K}\right\}} \beta_{\left\{\theta_{1}, \ldots, \theta_{K K}\right\}} \cdot \mathrm{B}_{\left\{\theta_{1}, \ldots, \theta_{K}\right\}}^{-1} \cdot \beta_{\left\{\theta_{1}, \ldots, \theta_{K}\right\}}^{T}
$$

where

$$
\beta_{\left\{\theta_{1}, \ldots, \theta_{K}\right\}}=\left[\boldsymbol{\theta}_{1}-\boldsymbol{\theta}, \ldots, \boldsymbol{\theta}_{K}-\boldsymbol{\theta}\right]
$$

and

$$
\left(\mathrm{B}_{\left\{\boldsymbol{\theta}_{1}, \ldots, \theta_{K}\right\}}\right)_{i j}=E\left[\frac{p\left(\mathbf{R} ; \boldsymbol{\theta}_{j}\right)}{p(\mathbf{R} ; \boldsymbol{\theta})} ; \boldsymbol{\theta}_{i}\right]-1 .
$$


This is also known as the Barankin-type bound [11].

The CRB and Bhattacharyya bounds are small-error bounds, which bound the performance in the neighborhood of the true parameter. The CRB is the most popular one because it is usually fairly easy to evaluate. It can be asymptotically approached by the maximum likelihood estimator at sufficiently high signal-to-noise ratio or long observation time [43]. However, for a given observation time, there often exists an SNR threshold below which the MLE MSE departs from the bound. This is the socalled threshold phenomenon and is due to the globally ambiguous estimation output and usually associated with the non-linear parameter estimation problem [72].

In contrast, the Barankin-type bound is the large-error bound in that it attempts to capture the effect of ambiguity errors through a set of test points within the given parameter space. It is tighter than the CRB (that means better performance prediction) and has been used for analyzing the threshold phenomenon [82, 83]. However, it is not, well manageable in choosing the optimum set of test points (cf. Section 2.3 .2 for more discussions).

For the local bounds discussed, two issues need to be clarified. First, as mentioned before, a local bound is limited to unbiased estimates; but in the ambiguous region, estimates are often biased. Second, the local bound doesn't exploit any a priori information about the parameter. Since the noise dominates the low SNR region, the estimation error is determined by the a priori parameter distribution. Due to the above two factors, these local bounds cannot be bounded in themselves in the very low SNR region, resulting in impractical results. To obtain meaningful results for all regions of operation, a global bound is usually preferred.

The global bound is an extension of the previous results to the framework of Bayesian estimation. It assumes an a priori random parameter model and bounds the averaged mean square error over the a priori distribution, thus free from the bias assumption. As the main approach in this thesis, the global bound is reviewed and discussed in detail in Chapter 3. 


\subsection{Performance Bounds in the Matched-Field Problem}

Performance bounds have received considerable attentions from the beginning of the matched-field processing research. They help to understand the fundamental limitation in parameter estimation by combining the propagation physics, environmental uncertainties, source-receiver geometries, and signal structure and statistics [10]. Thus they provide baselines to which the performances of various ad hoc algorithms can be compared.

A performance bound is derived according to a pre-defined data model. There are several types of data models currently used in the matched-field problem. Although expressed in different ways, they may all be fitted into a general form: multiplying the source signal with a Green's function and summing with an additive noise term [10], that is,

$$
\mathbf{R}(f, \boldsymbol{\theta})=\tilde{b}(f) S_{s}(f) \mathbf{G}(f, \boldsymbol{\theta})+\mathbf{N}(f, \boldsymbol{\theta}), \quad f \in \Delta f,
$$

where

$\mathbf{R}(f, \boldsymbol{\theta})$ is the complex envelope of the received signal;

$\theta$ is the unknown source or environmental parameter set;

$\tilde{b}(f)$ is a random process incorporating amplitude and phase variability;

$S_{s}(f)$ is the Fourier transform of the source signal;

$\mathbf{G}(f, \theta)$ is a vector of Green's function for the propagation to the receiver array under the given source and channel configuration;

$\mathrm{N}(f, \theta)$ is a stationary noise vector; and

$\Delta f$ is the signal bandwidth. 
Both the deterministic and random models have been considered for the source signal, but usually the latter one is more appropriate. Under the random signal model, two different types of sources can be applied:

1. Coherent across frequencies. $\tilde{b}(f)=\tilde{b}$, a scalar random variable with variance $\sigma_{b}^{2} . S_{s}(f)$ is the source signal, which can be an $M$ sequence or $F M$ sweep.

2. Incoherent across frequencies. $S_{s}(f)$ is set to one and $\vec{b}(f)$ is chosen to have a power spectral density equal of the source, $S_{b}(f)=\left|S_{s}(f)\right|^{2}$.

Item 1 is a typical model in ocean acoustic tomography $[52,10]$, while item 2 is a typical model for matched-field processing or matched-field tomography [10], in which the source is a stationary random process.

Except for the noise tomography case $\left(S_{s}(f)=0\right)$, the noise is generally assumed to be independent of the chosen parameter with a spectral covariance matrix $\mathrm{K}_{n}(f)=T_{0} \mathrm{~S}_{n}(f)$, where $T_{0}$ is the observation time, ${ }^{4}$ and $\mathrm{S}_{n}(f)$ is the power spectral density estimate of the noise. This is reasonable since the total field environmentaldependence is often dominated by the signal environmental-dependence.

The random signal and noise are often assumed to be Gaussian; therefore, the observation covariance matrix is important in deriving the performance bound. Under the data model for MFP/MFT, the covariance matrix for the observation vector $\mathbf{R}(f, \boldsymbol{\theta})$ is given by:

$$
\mathrm{K}_{\mathbf{R}}(f, \boldsymbol{\theta})=E\left[\mathbf{R} \mathbf{R}^{\dagger}\right]=T_{0} S_{b}(f) \mathbf{G}(f, \theta) \mathbf{G}^{\dagger}(f, \theta)+T_{0} S_{n}(f)
$$

For the received signal with $M$ uncorrelated frequency components, the covariance matrix $\mathrm{K}_{\mathrm{R}}(\boldsymbol{\theta})$ is a block-diagonal matrix with $\mathrm{K}_{\mathrm{R}}(f, \boldsymbol{\theta})$ as the diagonal element.

\footnotetext{
${ }^{4}$ Introduction of $T_{0}$ is from the power spectral density estimation based on the periodogram of a time-limited wide-sense stationary random process [81].
} 


\subsubsection{Cramer-Rao Bound}

The Cramer-Rao bound has been well developed for matched-field methods $[9,47$, $54,10,63,18,19,67,27]$. Given the complex Gaussian observations, the $(i, j)$ th element of the Fisher information matrix is [9]:

$$
\left[\mathrm{J}_{D}\right]_{i j}(\boldsymbol{\theta})=\operatorname{Tr}\left[\mathrm{K}_{\mathbf{R}}^{-1}(\boldsymbol{\theta})\left(\frac{\partial}{\partial \theta_{i}} \mathrm{~K}_{\mathbf{R}}(\boldsymbol{\theta})\right) \mathrm{K}_{\mathbf{R}}^{-1}(\boldsymbol{\theta})\left(\frac{\partial}{\partial \theta_{j}} \mathbf{K}_{\mathbf{R}}(\boldsymbol{\theta})\right)\right] .
$$

For the MFP/MFT data model used here, it has the form [10, 27]:

$$
\begin{aligned}
{\left[J_{D}\right]_{i j}(\boldsymbol{\theta})=} & \sum_{m=1}^{M} S_{b}^{2}\left(f_{m}\right) \gamma\left(f_{m}, \boldsymbol{\theta}\right)\left(\operatorname{Re}\left[d^{2}\left(f_{m}, \boldsymbol{\theta}\right) l_{i, j}\left(f_{m}, \boldsymbol{\theta}\right)-l_{i}\left(f_{m}, \boldsymbol{\theta}\right) l_{j}^{\dagger}\left(f_{m}, \boldsymbol{\theta}\right)\right]\right. \\
& \left.+\gamma\left(f_{m}, \boldsymbol{\theta}\right) \operatorname{Re}\left[l_{i}\left(f_{m}, \boldsymbol{\theta}\right)\right] \operatorname{Re}\left[l_{j}\left(f_{m}, \boldsymbol{\theta}\right)\right]\right)
\end{aligned}
$$

where

$d^{2}\left(f_{m}, \boldsymbol{\theta}\right)=\mathbf{G}^{\dagger}\left(f_{m}, \boldsymbol{\theta}\right) \mathrm{S}_{n}^{-1}\left(f_{m}\right) \mathbf{G}\left(f_{m}, \boldsymbol{\theta}\right)$ is the signal-to-noise ratio for the Green's function referenced in the space of the additive noise;

$l_{i}\left(f_{m}, \boldsymbol{\theta}\right)=\mathrm{G}^{\dagger}\left(f_{m}, \boldsymbol{\theta}\right) \mathrm{S}_{n}^{-1}\left(f_{m}\right)\left(\frac{\partial}{\partial \theta_{i}} \mathrm{G}\left(f_{m}, \boldsymbol{\theta}\right)\right)$ is the measure of the mean of the parameter sensitivity in the same space;

$l_{i, j}\left(f_{m}, \boldsymbol{\theta}\right)=\left(\frac{\partial}{\partial \theta_{i}} \mathbf{G}^{\dagger}\left(f_{m}, \boldsymbol{\theta}\right)\right) \mathbf{S}_{n}^{-1}\left(f_{m}\right)\left(\frac{\partial}{\partial \theta_{j}} \mathbf{G}\left(f_{m}, \boldsymbol{\theta}\right)\right)$ is the measure of the convexity of the parameter sensitivity; and

$\gamma\left(f_{m}, \boldsymbol{\theta}\right)=2 /\left(1+S_{b}\left(f_{m}\right) d^{2}\left(f_{m}, \boldsymbol{\theta}\right)\right)$.

In many adaptive searching algorithms, the resolution represented by (2.8) is often used as the local parameter sensitivity measure to adjust the search space; therefore, the resolution inhomogeneity and parameter coupling introduced by the waveguide physics can affect the convergence performance of an adaptive algorithm significantly. Refs. [63] and [27] have applied the CRB to analyze these issues, and the results show that a careful choice of the parameter set is important for robust matched-field parameter estimation. 
In Refs. $[18,19]$, a universal wave approach to ocean acoustic tomography is proposed and a CRB is derived for sound speed field reconstruction. The derived results are used to determine the information required to invert the field for different scales of field inhomogeneities, and then set up a statistical basis for modal, ray and interference acoustic tomography.

Despite its simple analytic form, the CRB is merely a high-SNR and asymptotic bound. For low SNR and short observation time, the reliability of the CRB is always doubted $[10]$.

\subsubsection{Barankin Bound}

A few papers have addressed the threshold phenomenon in matched-field processing. Particularly, the Barankin bound has been applied to the problem of source localization. In Ref. [66], the random signal model is considered, and multiple snapshots of a single frequency component are used. The signal-to-noise ratio is added to the source and environmental parameter set, $\Psi$. Accordingly, the unknown parameter vector is given as $\theta \equiv\left[\Psi^{T}, S N R\right]^{T}$, and the data covariance matrix for each snapshot has a similar form in (2.15).

Under the given model, the Barankin bound in (2.11) has

$$
\left(\mathrm{B}_{\left\{\theta_{1}, \ldots, \theta_{K}\right\}}\right)_{i j}=\left(\mathrm{B}_{\left\{\theta_{1}, \ldots, \theta_{K}\right\}}^{\prime}\right)_{i j}-1
$$

with

$$
\left(\mathrm{B}^{\prime}{ }_{\left\{\boldsymbol{\theta}_{1}, \ldots, \boldsymbol{\theta}_{K}\right\}}\right)_{i j}=\frac{\mathrm{K}_{\mathbf{R}}\left(f_{0}, \boldsymbol{\theta}\right)}{\left|\mathrm{K}_{\mathbf{R}}\left(f_{0}, \boldsymbol{\theta}_{i}\right)\right|\left|\mathrm{K}_{\mathbf{R}}\left(f_{0}, \boldsymbol{\theta}_{j}\right)\right|\left|\mathrm{K}_{\mathbf{R}}^{-1}\left(f_{0}, \boldsymbol{\theta}_{i}\right)+\mathrm{K}_{\mathbf{R}}^{-1}\left(f_{0}, \boldsymbol{\theta}_{j}\right)-\mathrm{K}_{\mathbf{R}}^{-1}\left(f_{0}, \boldsymbol{\theta}\right)\right|} .
$$

$\mathrm{B}_{i j}^{\prime}$ measures the field similarity associated with the test point and the true parameter point. Clearly, to get a tighter bound, the test points should be selected so 
that

$$
\mathrm{K}_{\mathbf{R}}\left(f_{0}, \theta_{i}\right) \sim \mathrm{K}_{\mathrm{R}}\left(f_{0}, \theta\right)
$$

for $\theta_{i}$ different from $\theta$. These points actually correspond to the ambiguity points on an ambiguity surface. This could explain why the Barankin bound is frequently used for analyzing the threshold phenomenon.

In Ref. [66], the environmental uncertainties are represented by uncertainties in the modal horizontal wavenumber, and to save the computational effort, only the largest component of the uncertainties is retained as the environmental parameter in $\Psi$. The test points are selected based on the diagonal term of the matrix $B^{\prime}$. First, $J$ candidate test points are chosen around each sidelobe. Then, the $K$ test points yielding the lowest values in the diagonal of $B^{\prime}$ are selected. This searching-based approach may find some test points contributing most to the bound as long as the candidate points cover the entire ambiguity region, but obviously not in an optimal way.

The bound's evaluation results are tighter than the CRB and demonstrate a. threshold behavior. However, the predicted threshold SNR is $3-5 \mathrm{~dB}$ lower than the simulations using the maximum likelihood estimator and the bound is still far less tight in the transition region. As the SNR further decreases, the bound even diverges. These behaviors are attributed to the sub-optimality in choosing the test points and the local characterization of the bound as discussed in Section 2.2.1.

In addition, (2.19) holds if and only if

$$
\mathrm{K}_{\mathrm{R}}^{-1}\left(f_{0}, \theta_{i}\right)+\mathrm{K}_{\mathrm{R}}^{-1}\left(f_{0}, \theta_{j}\right)-\mathrm{K}_{\mathrm{R}}^{-1}\left(f_{0}, \theta\right)
$$

is a positive definite matrix. This condition significantly reduces the number of available test points and limits the application of the Barankin bound in the MFP/MFT problem. 


\subsection{Summary}

Overall, most current performance analyses in matched-field parameter estimation concentrate on the local bound, the CRB or the Barankin bound. As we discussed in Section 2.2.1, both of them require unbiased estimates and don't exploit any a priori information about the parameter. Moreover, the Barankin-type large-error bound is difficult to evaluate and even shows some instability in certain estimation problems [1]. In contrast, some global bounds assume a random parameter model with a known a priori distribution, which is consistent with the implementation of most current MFP/MFT algorithms in searching the parameter space. Besides, they bound the averaged mean square error over the parameter interval; if the local performance shows a small variation across the interval, a global large-error bound describes the local mean square error at each parameter point well. 


\section{Chapter 3}

\section{Bayesian Bounds in Matched-Field Parameter Estimation}

In this chapter, a general framework for performance analysis in Bayesian estimation is first introduced. Under this framework, a few Bayesian performance bounds are reviewed, and some of the new observations are also pointed out. The data model used in this thesis is then defined, and three Bayesian bounds (Bayesian Cramer-Rao bound, Weiss-Weinstein bound and Ziv-Zakai bound) are developed accordingly for the matched-field problem.

\subsection{Bayesian Performance Bounds}

In the framework of Bayesian estimation, the unknown parameters are treated as random variables. For a single parameter $\theta$, the mean square error is bounded from below by

$$
\epsilon^{2}=E\left[(\hat{\theta}(\mathbf{R})-\theta)^{2}\right] \geq \xi^{2}
$$

where the expectation is implemented with respect to both the observation and the parameter, and $\xi^{2}$ can be an arbitrary bound.

For the vector parameter case, the bound is usually a matrix, $\Xi$, bounding the 
MSE matrix as stated below:

$$
\Sigma=E\left[(\hat{\theta}(\mathbf{R})-\theta)(\hat{\theta}(\mathbf{R})-\theta)^{T}\right] \geq \Xi
$$

One can obtain the bound for each individual parameter by multiplying the corresponding matrix with a weighting vector a:

$$
\mathbf{a}^{T} \Sigma \mathbf{a} \geq \mathbf{a}^{T} \Xi \mathbf{a}
$$

For example, choosing $\mathbf{a}=\left[\begin{array}{llll}1 & 0 & 0 & \ldots\end{array}\right]^{T}$ gives the bound for the first parameter.

An off-diagonal term of $\Sigma$ is an indication of parameter coupling, which specifies how the error in estimation of one parameter correlates with the error in estimation of another parameter. To see how well this off-diagonal term is predicted by the bound, let us consider the case with two parameters. Because we must have

$$
|\Sigma-\Xi| \geq 0
$$

we have

$$
\left(\Sigma_{11}-\Xi_{11}\right) \cdot\left(\Sigma_{22}-\Xi_{22}\right) \geq\left|\Sigma_{12}-\Xi_{12}\right|^{2}
$$

If $\Xi_{11} \rightarrow \Sigma_{11}$ and $\Xi_{22} \rightarrow \Sigma_{22}$, then $\Xi_{12} \rightarrow \Sigma_{12}$. Otherwise, $\Xi_{12}$ and $\Sigma_{12}$ could differ significantly. Therefore, this coupling term is closely predicted only if the mean square error for each individual parameter is closely predicted by the bound (e.g., the CRB at high SNR).

Most Bayesian bounds, such as the Bayesian CRB and Weiss-Weinstein bound, can be derived from a general covariance inequality developed by Weiss and Weinstein [78]. To state this covariance inequality, we denote $\theta \equiv\left[\theta_{1}, \ldots, \theta_{N_{p}}\right]^{T}$ and specify a set of real functions of the observation and the parameter set, $\left\{\Psi_{j}(\mathbb{R}, \theta)\right\}_{j=1}^{N_{t}}\left(N_{t} \geq N_{p}\right)$, which satisfy the following conditions:

(a) $E\left[\Psi_{j}(\mathbb{R}, \theta) ; \mathbf{R}\right]=0$, for almost every $\mathbf{R}, \quad j=1, \ldots, N_{t}$; 
(b) $\left|E\left[\theta_{i} \Psi_{j}(\mathbf{R}, \theta)\right]\right|<\infty, \quad i=1, \ldots, N_{p}, j=1, \ldots, N_{i}$;

(c) The $N_{t} \times N_{t}$ matrix $Q$, defined by $[Q]_{i j}=E\left[\Psi_{i}(\mathbf{R}, \theta) \Psi_{j}(\mathbf{R}, \boldsymbol{\theta})\right]$, is positive definite.

Then the MSE matrix for vector parameter estimation is bounded by

$$
\Sigma \geq \mathrm{WQ}^{-1} \mathrm{~W}^{T}
$$

where $\mathrm{W}$ is an $N_{p} \times N_{t}$ matrix with its $(i, j)$ th component given by

$$
[\mathbf{W}]_{i j}=E\left[\theta_{i} \Psi_{j}(\mathbf{R}, \theta)\right]
$$

Thus a series of bounds is obtained by choosing different $\left\{\Psi_{j}(\mathbb{R}, \boldsymbol{\theta})\right\}_{j=1}^{N_{t}}$.

The first one of interest is $\Psi_{j}(\mathbb{R}, \theta)=\theta_{j}-E\left[\theta_{j} \mid \mathbf{R}\right]$. The resulting bound is

$$
\Sigma \geq E\left[(\boldsymbol{\theta}-E[\boldsymbol{\theta} \mid \mathbf{R}])(\boldsymbol{\theta}-E[\boldsymbol{\theta} \mid \mathbf{R}])^{T}\right]
$$

This corresponds to the conditional mean estimator, whose performance establishes the greatest lower bound for the Bayesian estimation problem [81]. Unfortunately, in general, even numerically a direct evaluation of this performance is very difficult. Some weaker but simpler lower bounds are of more interest.

One exception is the Ziv-Zakai bound (ZZB), which is not derived from the above covariance inequality. Instead, the ZZB is derived from the detection theory, and can only bound the mean square error for each individual parameter. The relationship between the ZZB and the other covariance inequality-based bounds has not yet been established [13].

\subsubsection{Bayesian Cramer-Rao Bound}

The Bayesian CRB is first formularized by Van Trees [72, pages 72-73]. It can also be derived from (3.6) by choosing 


$$
\Psi_{j}(\mathbf{R}, \theta)=\frac{\partial \ln p(\mathbf{R}, \boldsymbol{\theta})}{\partial \theta_{j}}, \quad j=1, \ldots, N_{p}
$$

where $p(\mathbf{R}, \boldsymbol{\theta})$ is the joint pdf of $\mathbf{R}$ and $\boldsymbol{\theta}$.

The BCRB states for the MSE matrix:

$$
\Sigma \geq \mathrm{J}_{T}^{-1}
$$

where $\mathrm{J}_{T}$ is the global Fisher information defined by

$$
\left[\mathbf{J}_{T}\right]_{i j}=-E\left[\frac{\partial^{2} \ln p(\mathbf{R}, \boldsymbol{\theta})}{\partial \theta_{i} \partial \theta_{j}}\right]
$$

$p(\mathbb{R}, \boldsymbol{\theta})$ is the product of the prior probability density, $p(\boldsymbol{\theta})$, and the conditional probability density, $p(\mathbf{R} \mid \boldsymbol{\theta})$, so the global Fisher information, $\mathrm{J}_{T}$, can be further decomposed into two terms:

$$
\begin{aligned}
{\left[\mathrm{J}_{T}\right]_{i j} } & =-E\left[\frac{\partial^{2} \ln p(\mathbf{R} \mid \boldsymbol{\theta})}{\partial \theta_{i} \partial \theta_{j}}\right]-E\left[\frac{\partial^{2} \ln p(\boldsymbol{\theta})}{\partial \theta_{i} \partial \theta_{j}}\right] \\
& =\int_{\Theta}\left[\mathrm{J}_{D}\right]_{i j}(\boldsymbol{\theta}) \cdot p(\boldsymbol{\theta}) d \boldsymbol{\theta}+\left[\mathrm{J}_{A}\right]_{i j}
\end{aligned}
$$

where $J_{D}(\theta)$ is the local Fisher information at $\theta$ and $J_{A}$ is the a priori parameter information term.

It is well known [43] that for non-random parameter estimation, the local CRB is achieved by the MLE at high SNR or long observation time. In other words, considering the single parameter case, we have asymptotically for the MLE mean square error

$$
\epsilon^{2}(\theta)=J_{D}^{-1}(\theta)
$$

For the random parameter case, this is not always true. To demonstrate this, we assume a discrete set of parameter values, $\left\{\theta_{i}\right\}$, and note that the MLE mean square 
error is averaged over the parameter space, i.e.,

$$
\begin{aligned}
\epsilon^{2} & =\sum_{i} \epsilon^{2}\left(\theta_{i}\right) \cdot p\left(\theta_{i}\right) \\
& =\sum_{i} J_{D}^{-1}\left(\theta_{i}\right) \cdot p\left(\theta_{i}\right) .
\end{aligned}
$$

We have used (3.13) in the second equality. In the asymptotic region, the a priori term can be ignored and the BCRB is specified by

$$
\xi^{2}=\frac{1}{\sum_{i} J_{D}\left(\theta_{i}\right) \cdot p\left(\theta_{i}\right)}
$$

It can be shown that (3.14) is greater than (3.15) [38, page 130, and note that

$\left.\sum_{i} p\left(\theta_{i}\right)=1\right]$. They are equal if and only if $J_{D}\left(\theta_{i}\right)$ is not a function of $\theta_{i}$. This is an additional condition to achieve the Bayesian CRB using the Bayesian MLE. For the usual time-delay/bearing estimation problem, this condition is often satisfied.

Note that the BCRB requires $p(\mathbf{R}, \boldsymbol{\theta})$ be absolutely continuous with respect to $\boldsymbol{\theta}$ for almost every $\mathbf{R}$ in the observation space. Extensions of (3.9) to high-order derivatives and finite difference lead to the global Bhattacharyya bound and the BobrovskyZakai bound, respectively, but subject to more strict regularity conditions [79]. To satisfy those conditions, a random parameter model with Gaussian distribution is preferred. Otherwise, some special smoothing processing is a must, as discussed in Section 6.1.1.

For low SNR and short observation time, since the BCRB cannot account for the sidelobe effects, it often gives too optimistic performance prediction. To predict the large error performance in this case, a more appropriate bound is desired.

\subsubsection{Weiss-Weinstein Bound}

Weiss and Weinstein proposed the following choice of $\Psi_{j}(\mathbf{R}, \boldsymbol{\theta})[78]$ :

$$
\Psi_{j}(\mathbf{R}, \boldsymbol{\theta})=L^{s_{j}}\left(\mathbf{R} ; \boldsymbol{\theta}+\mathbf{h}_{j}, \boldsymbol{\theta}\right)-L^{1-s_{j}}\left(\mathbf{R} ; \boldsymbol{\theta}-\mathbf{h}_{j}, \theta\right), \quad j=1, \ldots, N_{t},
$$


where

$$
L\left(\mathbf{R} ; \boldsymbol{\theta}_{1}, \boldsymbol{\theta}_{2}\right) \equiv p\left(\mathbf{R}, \boldsymbol{\theta}_{1}\right) / p\left(\mathbf{R}, \boldsymbol{\theta}_{2}\right)
$$

and $\mathbf{h}_{j}$ is called the $j$ th test point in the parameter space.

The Weiss-Weinstein bound is then obtained by substituting (3.16) into (3.6), which states

$$
\Sigma \geq \max _{\left\{s_{j}, \mathbf{h}_{j}\right\}} \mathrm{HQ}^{-1} \mathrm{H}^{T}
$$

where $\mathrm{H}=\left[\mathbf{h}_{1}, \ldots, \mathbf{h}_{N_{t}}\right]$ is an $N_{p} \times N_{t}$ matrix of vector parameter test points, and the $(i, j)$ th element of the $N_{t} \times N_{t}$ matrix $\mathrm{Q}$ is given by

$$
\begin{aligned}
& \quad[\mathrm{Q}]_{i j}= \\
& \frac{E\left[\left(L^{s_{i}}\left(\mathbf{R} ; \boldsymbol{\theta}+\mathbf{h}_{i}, \boldsymbol{\theta}\right)-L^{1-s_{i}}\left(\mathbf{R} ; \boldsymbol{\theta}-\mathbf{h}_{i}, \boldsymbol{\theta}\right)\right)\left(L^{s_{j}}\left(\mathbf{R} ; \boldsymbol{\theta}+\mathbf{h}_{j}, \boldsymbol{\theta}\right)-L^{1-s_{j}}\left(\mathbf{R} ; \boldsymbol{\theta}-\mathbf{h}_{j}, \boldsymbol{\theta}\right)\right)\right]}{E\left[L^{s_{i}}\left(\mathbf{R} ; \boldsymbol{\theta}+\mathbf{h}_{i}, \boldsymbol{\theta}\right)\right] E\left[L^{s_{j}}\left(\mathbf{R} ; \boldsymbol{\theta}+\mathbf{h}_{j}, \boldsymbol{\theta}\right)\right]}
\end{aligned}
$$

For a special case of $s_{j}=1 / 2, j=1, \ldots, N_{t}$ (which is actually required for a zero-mean Gaussian data model as discussed in Section 3.2.3), the matrix Q can be expressed as

$$
[\mathrm{Q}]_{i j}=2 \cdot \frac{\exp \left\{\mu\left(1 / 2, \mathbf{h}_{i}-\mathbf{h}_{j}\right)\right\}-\exp \left\{\mu\left(1 / 2, \mathbf{h}_{i}+\mathbf{h}_{j}\right)\right\}}{\exp \left\{\mu\left(1 / 2, \mathbf{h}_{i}\right)\right\} \cdot \exp \left\{\mu\left(1 / 2, \mathbf{h}_{j}\right)\right\}}
$$

and $\mu(s, \mathbf{h})$ is defined by

$$
\mu(s, \mathbf{h})=\ln \int_{\Omega} d \mathbf{R} \int_{\Theta} d \theta p^{1-s}(\mathbf{R}, \boldsymbol{\theta}) p^{s}(\mathbf{R}, \boldsymbol{\theta}+\mathbf{h})
$$

The WWB has several advantages over the previous bounds. First, it is free from regularity conditions on the data probability model. Second, it is shown in [79] that the BCRB is a special case of the WWB with $\mathbf{h}_{j}$ going to zero along the $j$ th 
dimension. 'Therefore, by maximizing the bound with respect to the test points, we are sure to obtain a tighter bound than the BCRB.

A few papers have applied the WWB to time-delay/bearing estimation problems. In Ref. [79], the bound is applied to estimation of the time-of-arrival of a pulse in the additive random noise. The performance at lower SNR levels is accurately predicted by the bound. At higher SNR levels, the bound is shown to be tighter than the Barankin bound. Ref. [79] also applies the WWB to two-channel time-delay estimation. The results show that the threshold SNR predicted by the WWB is much higher than that by the Chapman-Robbins bound. The bound also shows a similar performance to the Ziv-Zakai-type bound (cf. Section 3.1.3), which is close to that of the cross-correlation estimation with sufficiently large time-bandwidth product. It is interesting to observe that a tight bound is obtained by the choice of $h$ equal to the period of the quasi-periodic correlation function. This suggests that the WWB could help to analyze the ambiguity behavior in the problem.

In Ref. [29], the WWB is applied to estimation of the direction-of-arrival using a two-dimensional antenna array and shows a tighter result using the WWB than using the CRB. Ref. [14] compares several different bounds in the bearing estimation problem. The results show that the Weiss-Weinstein bound and Ziv-Zakai bound well predict the behavior of the maximum likelihood estimator in all SNR regions while the Barankin bound and particularly the CRB fail in the low SNR region.

\subsubsection{Ziv-Zakai Bound}

In the ambiguity region, the estimation problem can be decomposed into (1) interval detection and (2) parameter estimation within the chosen interval [72]. This has encouraged people to resort to detection performance analysis for development of large error bounds in parameter estimation. For example, the Ziv-Zakai bound (ZZB) [84, $16,22]$ is derived on the basis of the probability of deciding correctly between two hypotheses, $H_{0}$ and $H_{1}$, corresponding to two possible parameter sets, $\theta$ and $\theta+\Delta$,

\footnotetext{
${ }^{1}$ Delong [29] later has shown this is not true if the parameter distribution, $p(\theta)$, has a compact support and is not zero at the end points.
} 


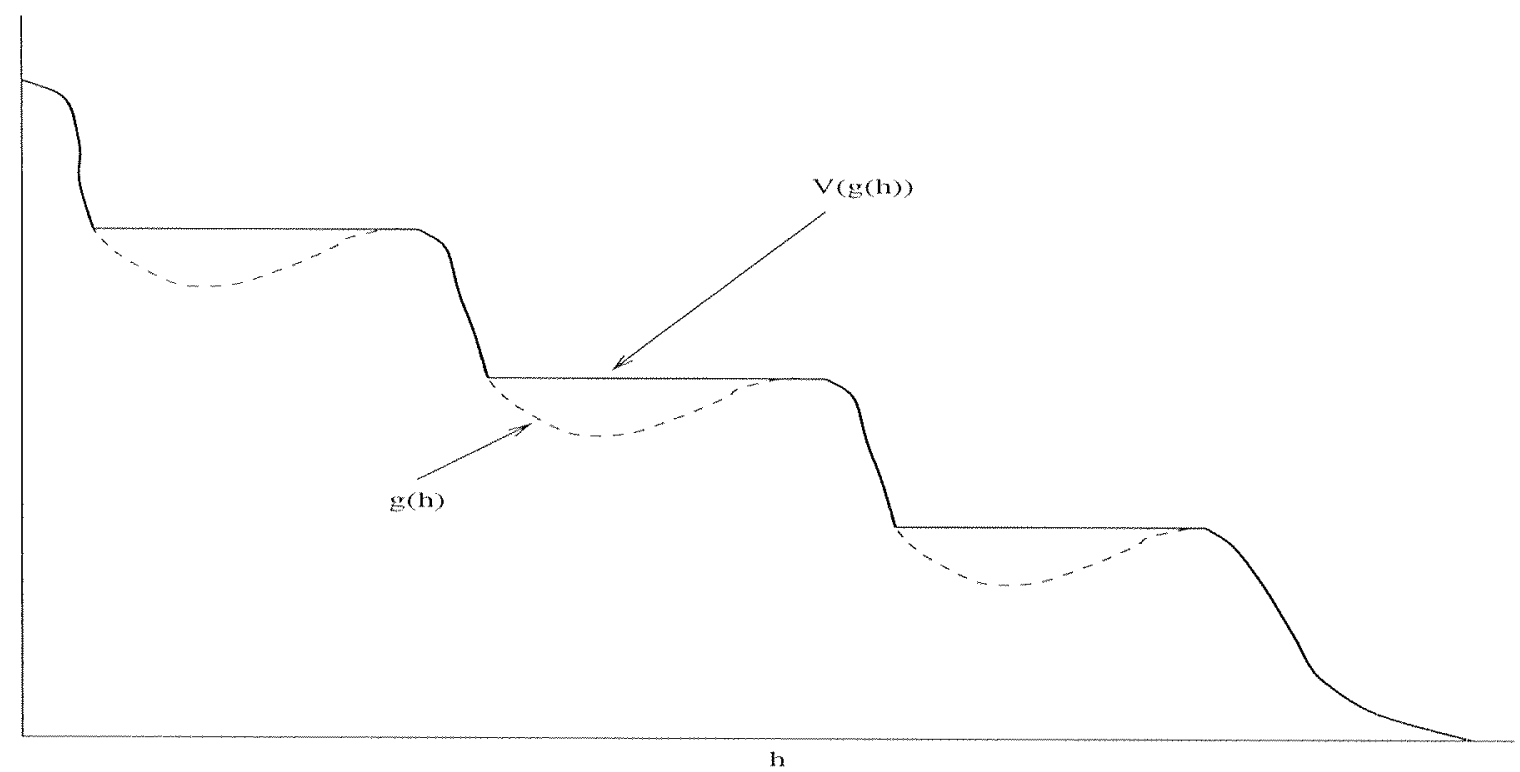

Figure 3-1: Valley filling function.

respectively. $\Delta$ can be considered as the vector parameter perturbation.

For a single parameter uniformly distributed in $[-\tau, \tau]$, the ZZB states [22]:

$$
\epsilon^{2} \geq \frac{1}{2 \tau} \int_{0}^{2 \tau} \delta \cdot d \delta \int_{-\tau}^{\tau-\delta} P_{e}(\theta, \theta+\delta) \cdot d \theta
$$

where $P_{e}(\theta, \theta+\delta)$ is the minimum achievable probability of error in determining the true parameter value between $\theta$ and $\theta+\delta$ associated with the likelihood ratio test [72].

Observing that $\int_{-\tau}^{\tau-\delta} P_{e}(\theta, \theta+\delta) d \theta$ is a non-increasing function of $\delta$, Bellini-Tartara proposed an improved bound [16]:

$$
\epsilon^{2} \geq \frac{1}{2 \tau} \int_{0}^{2 \tau} \delta \cdot V\left(\int_{-\tau}^{\tau-\delta} P_{e}(\theta, \theta+\delta) \cdot d \theta\right) \cdot d \delta
$$

where $V(\cdot)$ is a non-increasing function of $\delta$ obtained by filling the valleys in the bracketed function. As shown in Fig. 3-1, if the bracketed function has a significant oscillatory structure (e.g., in an ambiguity-prone problem), this bound could be much tighter than the original one.

Bell extended the Bellini-Tartara bound to arbitrarily distributed vector parameter estimation $[13,14,15]$. For any $N_{p}$-dimensional vector a, the extended ZZB 
states

$$
\mathbf{a}^{T} \Sigma \mathbf{a} \geq \int_{0}^{\infty} \delta \cdot V\left(\max _{\Delta: \mathrm{a}^{T} \Delta=\delta} \int_{\Theta} \min [p(\boldsymbol{\theta}), p(\boldsymbol{\theta}+\boldsymbol{\Delta})] \cdot P_{e}(\boldsymbol{\theta}, \boldsymbol{\theta}+\boldsymbol{\Delta}) d \boldsymbol{\theta}\right) \cdot d \delta
$$

Note that $P_{e}(\cdot)$ is defined for equally likely binary hypotheses detection.

$P_{e}(\theta, \theta+\Delta)$ is the most important factor in evaluating the ZZB, which determines the validity and tightness of the bound. In the context of parameter estimation and for a uniform parameter distribution, we have

$$
P_{e}(\boldsymbol{\theta}, \boldsymbol{\theta}+\Delta)=\min \left(\frac{1}{2} \cdot P_{e}(\boldsymbol{\theta}+\Delta \mid \boldsymbol{\theta})+\frac{1}{2} \cdot P_{e}(\boldsymbol{\theta} \mid \boldsymbol{\theta}+\Delta)\right)
$$

where $P_{e}(\theta+\Delta \mid \theta)$ is the probability that, given the true parameter at $\theta$, the test decision is $\theta+\Delta$, and $P_{e}(\boldsymbol{\theta} \mid \boldsymbol{\theta}+\Delta)$ is the error probability given that the true parameter is $\theta+\Delta$.

There have been considerable efforts for analyzing the detection performances under different data models $[72,13]$. However, except for some simple cases, direct calculation of $P_{e}(\boldsymbol{\theta}, \boldsymbol{\theta}+\boldsymbol{\Delta})$ is difficult. Some approximations or bounds have been developed for this error probability. A typical approximation derived from the Chernoff formula $[72]$ is

$$
\begin{aligned}
P_{e}(\boldsymbol{\theta}, \boldsymbol{\theta}+\Delta) \approx & \frac{1}{2} e^{\mu\left(s_{m}\right)+\frac{s_{m}^{2}}{2} \ddot{\mu}\left(s_{m}\right)} \cdot \operatorname{erfc}\left(s_{m} \sqrt{\ddot{\mu}\left(s_{m}\right)}\right) \\
& +\frac{1}{2} e^{\mu\left(s_{m}\right)+\frac{\left(1-s_{m}\right)^{2}}{2} \ddot{\mu}\left(s_{m}\right)} \cdot \operatorname{erfc}\left(\left(1-s_{m}\right) \sqrt{\left.\ddot{\mu}\left(s_{m}\right)\right)},\right.
\end{aligned}
$$

where $\operatorname{erfc}(\cdot)$ is the complement error function [3]. $\mu(s)$ is the semi-invariant moment generating function defined by

$$
\mu(s)=\ln \int_{\Omega}\left(p\left(\mathbf{R} / H_{1}\right)\right)^{s}\left(p\left(\mathbf{R} / H_{0}\right)\right)^{1-s} d \mathbf{R}
$$

where $p\left(\mathbb{R} / H_{i}\right)$ is the pdf of the observation under the corresponding hypothesis. $s_{m}$ is then chosen by $\dot{\mu}\left(s_{m}\right)=0$. Note that $\mu(s, h)$ in $(3.21)$, which includes the effect of random parameter distribution, can be considered as a generalization of (3.27). 
The function $\mu(s)$ plays an important role in performance analysis of the detection problem [72]. Many results for the error terms are expressed in terms of $\mu(s)$. (3.26) is a typical example developed from the central limit theorem, which is valid when the observation components, $\mathbb{R}_{l}, l=1, \ldots, L$, are independent random variables with finite means and variances. It can also be derived using the Edgeworth series representation of the underlying probability density function $[73,24]$.

The ZZB has been widely applied to time-delay and bearing estimation $[80,77,14]$. These applications have demonstrated that the ZZB can well specify the performance limits in all SNR regions. The expense paid is the complexity in analysis and computation. Although in a few special cases a closed-form analytical expression of the bound is available $[80,14]$, this is generally impossible, for example, in the matchedfield problem.

It is worth noting that a Bayesian bound bounds the global mean square error of an estimator. Therefore, if the errors associated with some parameter points are obviously larger than those at other parameter points, they will strongly influence the averaged error.

\subsection{Applications to Matched-Field Parameter Es- timation}

The Bayesian-type bound has not been applied to the matched-field problem yet. In this section, we derived the BCRB, WWB and ZZB using the defined MFP/MFT data model.

\subsubsection{Data Model}

As before, we denote the unknown parameter set by a vector $\theta$. Typically, this parameter set may include source position (range, depth and bearing) and/or environmental parameters (e.g., bathymetry, sound propagation speed, attenuation, and density). The dimensionality of this parameter space can be very high, particularly 
for a range-dependent environment, but the influence of each parameter on sound propagation can differ dramatically. So one often resorts to a simplified but representative model when some prior site information is available.

For the usual matched-field localization and tomography problem, the source is a stationary random process. When the observation time is long enough (so that the window effect in spectral estimation can be ignored), individual DFT bins of the received signal are uncorrelated with each other [81]. Therefore this random source is incoherent across its frequency band. We further assume a number of snapshots are available for each frequency, where the number of snapshots is determined by the observation time and the correlation time of the source process [50].

Suppose we have $L$ independent measurements for each of $M$ frequencies, $f_{m}$, $m=1,2, \ldots, M$. The complex envelope of the received signal using the notation in Section 2.3 is expressed as $[10]$ :

$$
\mathbf{R}_{l}\left(f_{m}, \boldsymbol{\theta}\right)=\tilde{b}_{l}\left(f_{m}\right) \mathbf{G}\left(f_{m}, \theta\right)+\mathbf{N}_{l}\left(f_{m}\right), \quad l=1, \ldots, L, m=1, \ldots, M
$$

Obviously, $\mathbf{R}_{l}\left(f_{m}, \boldsymbol{\theta}\right)$ is an $N \times 1$ vector representing the $l$ th snapshot of the $m$ th frequency component, and $N$ is the number of sensors of the receiver array.

Both the signal and noise terms are now assumed to follow a zero-mean Gaussian distribution. The covariance matrix for $\mathbf{R}_{l}\left(f_{m}, \boldsymbol{\theta}\right)$ is given by

$$
\mathrm{K}_{\mathbf{R}}\left(f_{m}, \boldsymbol{\theta}\right)=\sigma_{b}^{2}\left(f_{m}\right) \mathbf{G}\left(f_{m}, \boldsymbol{\theta}\right) \mathbf{G}^{\dagger}\left(f_{m}, \boldsymbol{\theta}\right)+\sigma_{n}^{2}\left(f_{m}\right) \mathrm{I}
$$

where $\sigma_{b}^{2}\left(f_{m}\right)=T_{f} S_{b}\left(f_{m}\right)$ is the signal variance, $\sigma_{n}^{2}\left(f_{m}\right)=T_{f} S_{n}\left(f_{m}\right)$ is the variance of the white noise process, and $T_{f}$ is the FFT duration.

Denote

$$
\mathbf{R}=\left[\begin{array}{lllllllll}
\mathbf{R}_{1}^{T}\left(f_{1}\right) & \ldots & \mathbf{R}_{1}^{T}\left(f_{M}\right) & \ldots & \ldots & \ldots & \mathbb{R}_{L}^{T}\left(f_{1}\right) & \ldots & \mathbf{R}_{L}^{T}\left(f_{M}\right)
\end{array}\right]^{T}
$$

Obviously, $\mathbf{R}$ is an $(N \times M \times L) \times 1$ column vector. $\mathbf{R}_{l}\left(f_{m}\right), l=1, \ldots, L, m=1, \ldots, M$, are uncorrelated across frequencies and snapshots, so the covariance matrix is a block 
diagonal matrix,

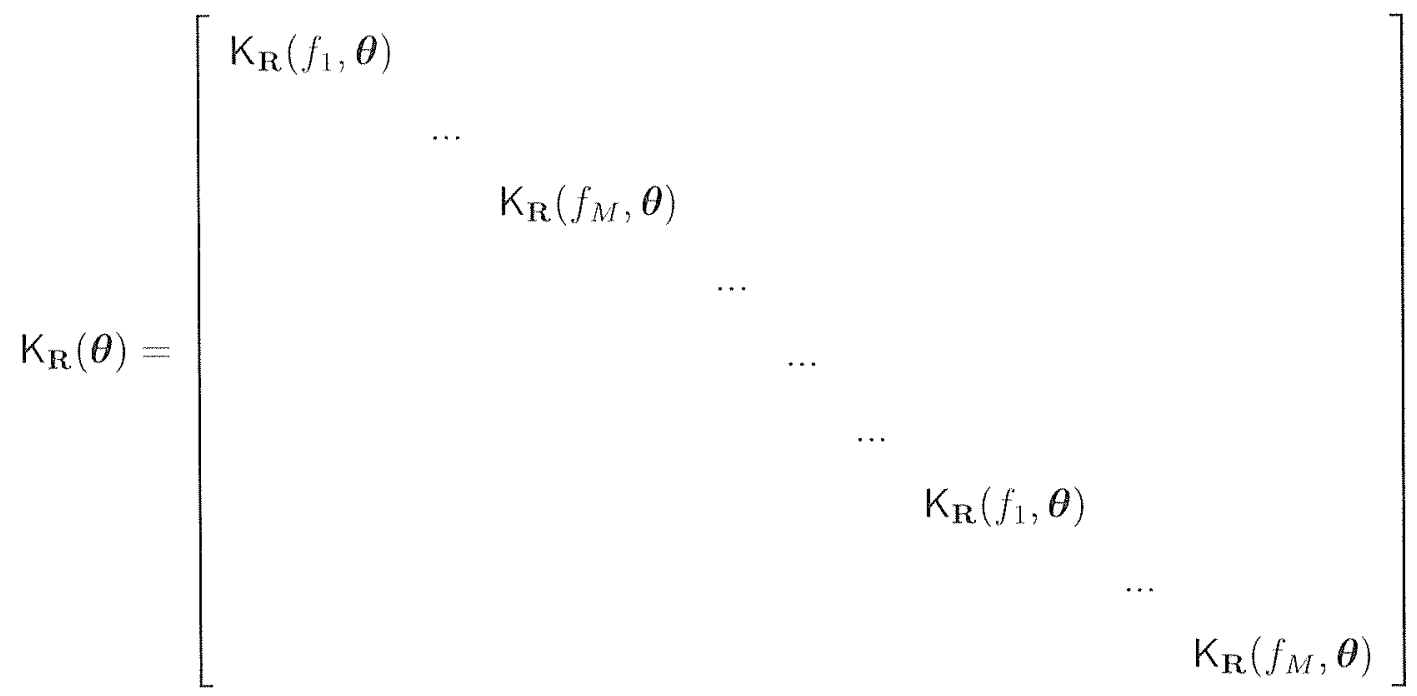

and the conditional probability density function of $\mathbf{R}$ is

$$
\begin{aligned}
p(\mathbf{R} \mid \boldsymbol{\theta}) & =\frac{1}{\left|\pi \mathrm{K}_{\mathbf{R}}(\boldsymbol{\theta})\right|} \exp \left(-\mathbf{R}^{\dagger} \mathrm{K}_{\mathbf{R}}^{-1}(\boldsymbol{\theta}) \mathbf{R}\right) \\
& =\frac{1}{\prod_{m=1}^{M}\left|\pi \mathrm{K}_{\mathbf{R}}\left(f_{m}, \boldsymbol{\theta}\right)\right|^{L}} \prod_{m=1}^{M} \prod_{l=1}^{L} \exp \left(-\mathbf{R}_{l}^{\dagger}\left(f_{m}\right) \mathrm{K}_{\mathbf{R}}^{-1}\left(f_{m}, \boldsymbol{\theta}\right) \mathbf{R}_{l}\left(f_{m}\right)\right)
\end{aligned}
$$

\subsubsection{Bayesian Cramer-Rao Bound}

The CRB is specified by the inverse of the Fisher information. An expression of the local Fisher information, $\mathrm{J}_{D}(\boldsymbol{\theta})$, is available based on the results in [10]:

$$
\begin{aligned}
{\left[\mathrm{J}_{D}\right]_{i j}(\theta)=} & L \sum_{m=1}^{M}\left[S _ { b } ^ { 2 } ( f _ { m } ) \gamma ( f _ { m } , \theta ) \left(\operatorname { R e } \left[d^{2}\left(f_{m}, \theta\right) l_{i, j}\left(f_{m}, \theta\right)\right.\right.\right. \\
& \left.\left.\left.-l_{i}\left(f_{m}, \theta\right) l_{j}^{\dagger}\left(f_{m}, \theta\right)\right]+\gamma\left(f_{m}, \theta\right) \operatorname{Re}\left[l_{i}\left(f_{m}, \theta\right)\right] \operatorname{Re}\left[l_{j}\left(f_{m}, \theta\right)\right]\right)\right],
\end{aligned}
$$


where $d^{2}\left(f_{m}, \theta\right), l_{i}\left(f_{m}, \theta\right), l_{i, j}\left(f_{m}, \theta\right)$, and $\gamma\left(f_{m}, \theta\right)$ are defined below (2.17) in Section 2.3.1.

This local Fisher information depends on specific parameter values. As a result, the MLE mean square error at high SNR is often a bit higher than the bound's prediction, and the difference is an indication of the local performance variation.

\subsubsection{Weiss-Weinstein Bound}

Calculation of the WWB relies upon evaluating $\mu(s, \mathbf{h})$ in $(3.21)$. For the zero-mean Gaussian signal and noise model in Section 3.2.1, we have

$$
\begin{aligned}
\mu(s, \mathbf{h}) & =\ln \left[\int_{\Theta} d \boldsymbol{\theta} \frac{p^{s}(\boldsymbol{\theta}+\mathbf{h}) p^{1-s}(\boldsymbol{\theta})}{\left|\mathrm{K}_{\mathbf{R}}(\boldsymbol{\theta}+\mathbf{h})\right| s\left|\mathrm{~K}_{\mathbf{R}}(\boldsymbol{\theta})\right|^{1-s}\left|s \mathrm{~K}_{\mathbf{R}}^{-1}(\boldsymbol{\theta}+\mathbf{h})+(1-s) \mathrm{K}_{\mathbf{R}}^{-1}(\boldsymbol{\theta})\right|}\right] \\
& =\ln \left[\int_{\Theta} d \boldsymbol{\theta} \frac{p^{s}(\boldsymbol{\theta}+\mathbf{h}) p^{1-s}(\boldsymbol{\theta})}{\prod_{m=1}^{M} \eta^{L}\left(f_{m}, s, \boldsymbol{\theta}, \mathbf{h}\right)}\right]
\end{aligned}
$$

where $\eta\left(f_{m}, s, \boldsymbol{\theta}, \mathbf{h}\right)$ is defined by

$$
\begin{aligned}
& \eta\left(f_{m}, s, \boldsymbol{\theta}, \mathbf{h}\right)= \\
& \left|\mathrm{K}_{\mathbf{R}}\left(f_{m}, \boldsymbol{\theta}+\mathbf{h}\right)\right|^{s}\left|\mathrm{~K}_{\mathbf{R}}\left(f_{m}, \boldsymbol{\theta}\right)\right|^{1-s}\left|s \mathrm{~K}_{\mathbf{R}}^{-1}\left(f_{m}, \boldsymbol{\theta}+\mathbf{h}\right)+(1-s) \mathrm{K}_{\mathbf{R}}^{-1}\left(f_{m}, \boldsymbol{\theta}\right)\right|
\end{aligned}
$$

The detailed derivations are given in Appendix A.

In the way to (3.34) (which is the denominator in (3.19)), we need

$$
s K_{\mathbf{R}}^{-1}(\theta+\mathbf{h})+(1-s) K_{\mathbf{R}}^{-1}(\theta)
$$

to be positive definite. Since both $\mathrm{K}_{\mathbf{R}}(\theta)$ and $\mathrm{K}_{\mathrm{R}}(\boldsymbol{\theta}+\mathrm{h})$ are positive definite matrices, for $0 \leq s \leq 1$, the positive definiteness of (3.37) is guaranteed. Similarly, in deriving the numerator of (3.19), we must have

$$
2 s \mathrm{~K}_{\mathbf{R}}^{-1}(\boldsymbol{\theta}+\mathbf{h})+(1-2 s) \mathrm{K}_{\mathrm{R}}^{-1}(\boldsymbol{\theta})
$$


and

$$
(2-2 s) \mathrm{K}_{\mathbf{R}}^{-1}(\boldsymbol{\theta}+\mathbf{h})+(2 s-1) \mathrm{K}_{\mathrm{R}}^{-1}(\boldsymbol{\theta})
$$

to be positive definite. The former one requires $s \leq 1 / 2$ and the latter one requires $s \geq 1 / 2$. As a result, the only choice of $s$ capable of securing the bound's calculation is $1 / 2$. This constraint could hurt the tightness of the WWB.

From $(3.35), \eta\left(f_{m}, s, \boldsymbol{\theta}, \mathbf{h}\right)$ is actually the basic computational component in (3.18), and can be expressed in terms of the source signal variance, the Green's function, and the noise variance, based on the eigen-decomposition of the covariance matrix. Define

$$
\begin{gathered}
A\left(f_{m}, \boldsymbol{\theta}\right)=\frac{\sigma_{b}^{2}\left(f_{m}\right)\left\|\mathbf{G}\left(f_{m}, \boldsymbol{\theta}\right)\right\|^{2}}{\sigma_{b}^{2}\left(f_{m}\right)\left\|\mathbf{G}\left(f_{m}, \boldsymbol{\theta}\right)\right\|^{2}+\sigma_{n}^{2}\left(f_{m}\right)}, \\
B\left(f_{m}, \boldsymbol{\theta}, \mathbf{h}\right)=\frac{\sigma_{b}^{2}\left(f_{m}\right)\left\|\mathbf{G}\left(f_{m}, \boldsymbol{\theta}, \mathbf{h}\right)\right\|^{2}}{\sigma_{b}^{2}\left(f_{m}\right)\left\|\mathbf{G}\left(f_{m}, \boldsymbol{\theta}+\mathbf{h}\right)\right\|^{2}+\sigma_{n}^{2}\left(f_{m}\right)},
\end{gathered}
$$

and

$$
C\left(f_{m}, \boldsymbol{\theta}, \mathbf{h}\right)=\mathbf{g}^{\dagger}\left(f_{m}, \boldsymbol{\theta}\right) \mathbf{g}\left(f_{m}, \boldsymbol{\theta}+\mathbf{h}\right)
$$

where $\mathrm{g}\left(f_{m}, \boldsymbol{\theta}\right)$ is the normalized Green's function. Clearly $A$ and $B$ are determined by the signal-to-noise ratios under $\theta$ and $\theta+\mathbf{h}$, respectively, and $C$ defines a signal field correlation. We then have (cf. Appendix A)

$$
\eta\left(f_{m}, \boldsymbol{\theta}, \mathbf{h}\right)=\frac{1}{\sqrt{(1-A)(1-B)}} \cdot\left(1-\frac{A+B}{2}+\frac{A B}{4}\left(1-|C|^{2}\right)\right)
$$

where $s=1 / 2$ is removed from the argument list of $\eta$, and $\mu(1 / 2, \mathbf{h})$ is now

$$
\mu(1 / 2, \mathbf{h})=\ln \left[\int_{\Theta} d \boldsymbol{\theta} \frac{p^{1 / 2}(\boldsymbol{\theta}+\mathbf{h}) p^{1 / 2}(\boldsymbol{\theta})}{\prod_{m=1}^{M} \eta^{L}\left(f_{m}, \boldsymbol{\theta}, \mathbf{h}\right)}\right]
$$




\subsubsection{Ziv-Zakai Bound}

As mentioned before, the kernel of the ZZB is the minimum achievable probability of error, $P_{e}(\theta, \theta+\Delta)$. Appendix $\mathrm{C}$ gives detailed derivations of this error probability using single- and multiple-frequency component models, respectively. The results are summarized below. Due to the symmetry between $P_{e}(\theta \mid \theta+\Delta)$ and $P_{e}(\theta+\Delta \mid \theta)$, we only give the results for $P_{e}\left(\boldsymbol{\theta}_{1} \mid \boldsymbol{\theta}_{0}\right) \cdot P_{e}(\boldsymbol{\theta} \mid \boldsymbol{\theta}+\boldsymbol{\Delta})$ and $P_{e}(\boldsymbol{\theta}+\boldsymbol{\Delta} \mid \boldsymbol{\theta})$ are then obtained by replacing $\theta_{0}$ and $\theta_{1}$ accordingly.

For the case with single frequency component, this error probability is

$$
P_{e}\left(\boldsymbol{\theta}_{1} \mid \boldsymbol{\theta}_{0}\right)=1-\frac{\left(-\frac{\lambda_{1}}{\lambda_{2}}\right)^{L}}{\left(1-\frac{\lambda_{1}}{\lambda_{2}}\right)^{2 L-1}} \sum_{k=0}^{L-1}\left(\begin{array}{c}
2 L-1 \\
k+L
\end{array}\right) \cdot\left(-\frac{\lambda_{1}}{\lambda_{2}}\right)^{k}
$$

where

$$
\begin{aligned}
& \lambda_{1,2}\left(f_{0}, \boldsymbol{\theta}_{0}, \boldsymbol{\theta}_{1}\right)= \\
& \frac{1}{2} \cdot\left[-\sigma_{b}^{2}\left(f_{0}\right)\left\|\mathbf{G}\left(f_{0}, \boldsymbol{\theta}_{0}\right)\right\|^{2}\left(1-\left|\mathbf{g}^{\dagger}\left(f_{0}, \boldsymbol{\theta}_{1}\right) \mathbf{g}\left(f_{0}, \boldsymbol{\theta}_{0}\right)\right|^{2}\right) \mp \sqrt{D}\right],
\end{aligned}
$$

and

$$
\begin{aligned}
& D\left(f_{0}, \boldsymbol{\theta}_{0}, \boldsymbol{\theta}_{1}\right)= \\
& \left(\sigma_{b}^{2}\left(f_{0}\right)\left\|\mathbf{G}\left(f_{0}, \boldsymbol{\theta}_{0}\right)\right\|^{2}\left(1-\left|\mathbf{g}^{\dagger}\left(f_{0}, \boldsymbol{\theta}_{1}\right) \mathbf{g}\left(f_{0}, \boldsymbol{\theta}_{0}\right)\right|^{2}\right)+2 \sigma_{n}^{2}\left(f_{0}\right)\right)^{2} \\
& -4 \sigma_{n}^{4}\left(f_{0}\right)\left|\mathbf{g}^{\dagger}\left(f_{0}, \boldsymbol{\theta}_{1}\right) \mathbf{g}\left(f_{0}, \boldsymbol{\theta}_{0}\right)\right|^{2} .
\end{aligned}
$$

An alternative expression is available (cf. Appendix C), but (3.45) is more computationally efficient.

For the case with multiple frequency components, an exact error probability is also available

$$
P_{e}\left(\boldsymbol{\theta}_{1} \mid \boldsymbol{\theta}_{0}\right)=\sum_{m=1}^{M} \sum_{k=1}^{L} C_{m k+}\left(-\lambda_{2 m}\right)^{k}
$$


where

$$
\begin{gathered}
C_{m k+}=\left.\frac{1}{(L-k) !}\left[\frac{\partial^{L-k}}{\partial s^{L-k}}\left(\left(s-\frac{1}{\lambda_{2 m}}\right)^{L} \Phi_{l^{\prime}}\right)\right]\right|_{s=1 / \lambda_{2 m}}, \\
\Phi_{l^{\prime}}(s)=\prod_{m=1}^{M}\left(\frac{\frac{1}{\lambda_{1 m}}}{s-\frac{1}{\lambda_{1 m}}}\right)^{L}\left(\frac{\frac{1}{\lambda_{2 m}}}{s-\frac{1}{\lambda_{2 m}}}\right)^{L},
\end{gathered}
$$

and $\left\{\lambda_{1 m}\right\}$ and $\left\{\lambda_{2 m}\right\}, m=1, \ldots, M$, are solved using $(3.46,3.47)$ with each $f_{m}$ replacing $f_{0}$.

Note that $C_{m k+}$ is the partial fraction expansion coefficient for the moment generating function, $\Phi_{l^{\prime}}(s)$. For large $M$ and $L$, calculation of $C_{m k+}$ is rather timeconsuming. A hierarchy of approximations based on the moment generating function is obtained using the Edgeworth series. The first two terms in the series are given by

$$
P_{e}^{[1]}\left(\boldsymbol{\theta}_{1} \mid \boldsymbol{\theta}_{0}\right)=\exp \left(\mu\left(s_{m}\right)+\frac{s_{m}^{2} \ddot{\mu}\left(s_{m}\right)}{2}\right) \operatorname{erfc}\left(s_{m} \sqrt{\ddot{\mu}\left(s_{m}\right)}\right)
$$

and

$$
P_{e}^{[2]}\left(\boldsymbol{\theta}_{1} \mid \boldsymbol{\theta}_{0}\right)=-\frac{s_{m}^{3} \mu^{(3)}\left(s_{m}\right)}{6} P_{e}^{[1]}\left(\boldsymbol{\theta}_{1} \mid \boldsymbol{\theta}_{0}\right)+\frac{1-s_{m}^{2} \ddot{\mu}\left(s_{m}\right)}{6 \sqrt{2 \pi}} \frac{\mu^{(3)}\left(s_{m}\right)}{\left(\ddot{\mu}\left(s_{m}\right)\right)^{3 / 2}} \exp \left(\mu\left(s_{m}\right)\right),
$$

where

$$
\begin{gathered}
\mu(s)=-L \sum_{m=1}^{M} \ln \left[\left(1-s \lambda_{1 m}\right)\left(1-s \lambda_{2 m}\right)\right], \\
\ddot{\mu}(s)=L \sum_{m=1}^{M}\left[\left(\frac{\lambda_{1 m}}{1-s \lambda_{1 m}}\right)^{2}+\left(\frac{\lambda_{2 m}}{1-s \lambda_{2 m}}\right)^{2}\right],
\end{gathered}
$$


and

$$
\mu^{(3)}(s)=2 L \sum_{m=1}^{M}\left[\left(\frac{\lambda_{1 m}}{1-s \lambda_{1 m}}\right)^{3}+\left(\frac{\lambda_{2 m}}{1-s \lambda_{2 m}}\right)^{3}\right]
$$

The first term is actually the approximation used in (3.26). For the problem here, $s_{m}$ is obtained by solving

$$
\sum_{m=1}^{M}\left[\frac{\lambda_{1 m}}{1-s \lambda_{1 m}}+\frac{\lambda_{2 m}}{1-s \lambda_{2 m}}\right]=0
$$

and choosing the root between $1 / \min \left(\lambda_{1 m} m=1, \ldots, M\right)$ and $1 / \max \left(\lambda_{2 m} m=\right.$ $1, \ldots, M)$

\subsection{Summary}

In this chapter, we have first reviewed some of the current results regarding the Bayesian performance bounds, and then developed the Bayesian CRB, Weiss-Weinstein bound and Ziv-Zakai bound for the matched-field parameter estimation problem.

We notice that the bound's basic component, either $\eta\left(f_{m}, \boldsymbol{\theta}, \mathbf{h}\right)$ of the WWB or $P_{e}(\boldsymbol{\theta}, \boldsymbol{\theta}+\Delta)$ of the $\mathrm{ZZB}$, is determined by a signal ambiguity function term $\left(\left|\mathrm{g}^{\dagger}\left(f_{m}, \boldsymbol{\theta}_{1}\right) \mathrm{g}\left(f_{m}, \boldsymbol{\theta}_{0}\right)\right|^{2}\right)$ and a signal-to-noise ratio term $\left(\frac{\sigma_{b}^{2}\left(f_{m}\right) \cdot\left\|\mathbf{G}\left(f_{m}, \boldsymbol{\theta}\right)\right\|^{2}}{\sigma_{n}^{2}\left(f_{m}\right)}\right)$. In the next chapter, the bounds' behaviors are discussed in terms of the ambiguities at different SNR levels. 


\section{Chapter 4}

\section{Ambiguity Analysis}

The signal ambiguity function is an indicator of the invertibility of the signal field. When this function is multi-modal, in the presence of noise or other interferences, we probably will have some parameter estimates around the sidelobes. Therefore, issues on the output mainlobe width and sidelobe levels are very important for the development of any matched-field algorithm.

This chapter addresses various ambiguity issues under a typical shallow water environment shown in Fig. 4-1. It is chosen from the SWellEX (Shallow Water Evaluation Cell Experiments) experiment scenarios [17]. The water column has a downward refracting sound velocity profile on the top but is almost isovelocity in the bottom half. The bottom depth is assumed to be constant at $198 \mathrm{~m}$. The bottom is modeled as a 26-m silty-sand sediment layer overlying an 800-m mudstone layer above a Cretaceous sandstone basement [68]. A linear upward refracting compressional wave-speed profile is assumed for the sediment layer as well as the mudstone layer and a constant wave-speed for the basement. The density and compressional wave attenuation in each layer are assumed to be constant. A 16-sensor array has a 114-m aperture and spans the water column from the middle to the bottom. The sensor spacing is of half-wavelength at the chosen frequency, $f_{0}=101 \mathrm{~Hz}$. The field is computed using the KRAKEN normal modes model [56]. 


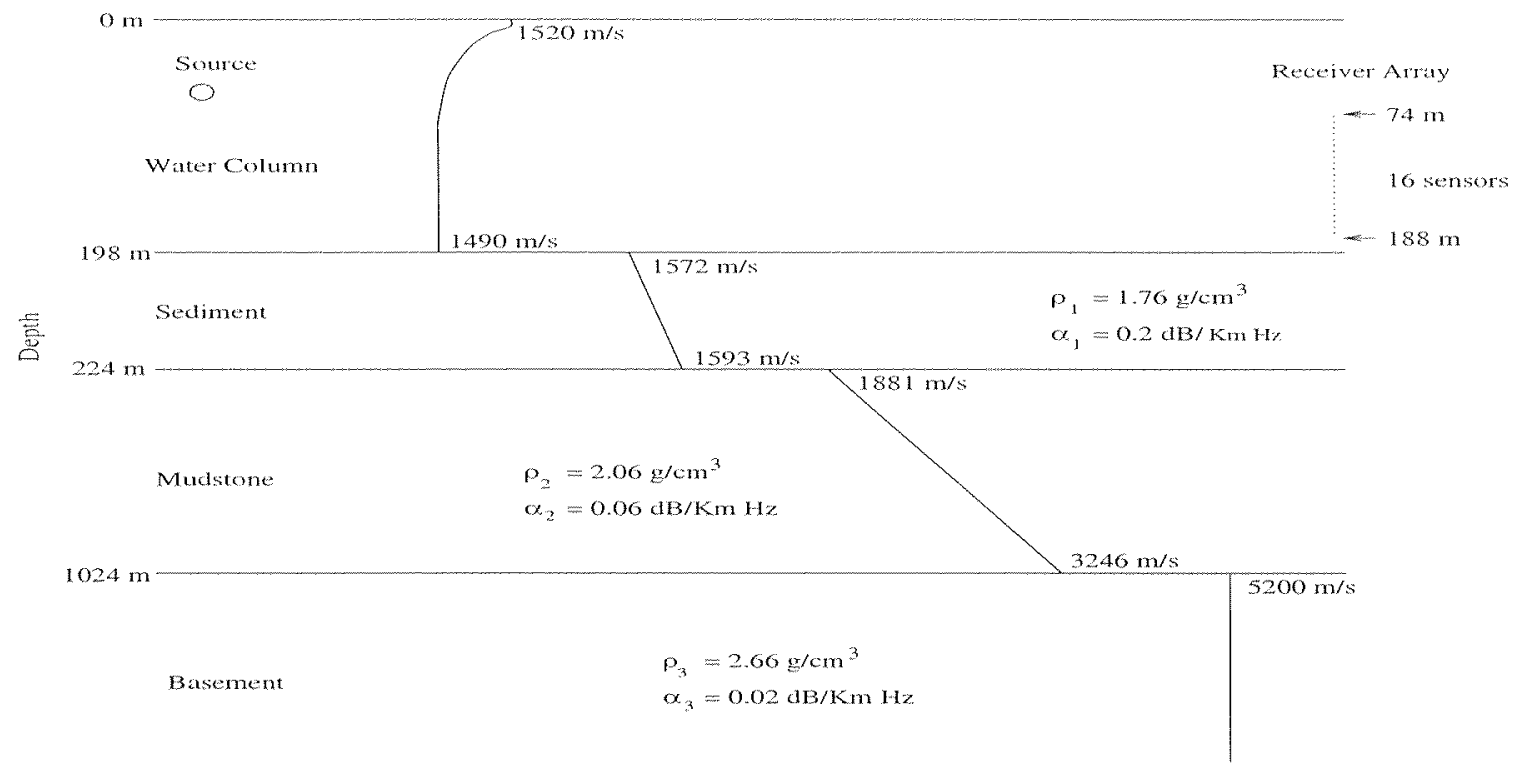

Figure 4-1: A shallow water environment with depth-dependent sound speed profile. Source and receiver configuration is also shown.

\subsection{Fundamentals of Ambiguity in the Matched- Field Problem}

Using the normal modes representation, the full-field signal space has a finite dimension. The signal field is uniquely determined in the absence of noise if (1) the number of sensors is more than the number of (significant) propagation modes; and (2) the sensor array has sufficient column spanning. Then for a limited number of parameters, the true parameter position is uniquely resolved as well from the peak of the field correlation. This field correlation, however, often demonstrates a multi-modal behavior.

The multi-modal ambiguity structure comes from the nonlinear dependence of the signal field on the embedded parameters. Recall from (2.2) that for a rangeindependent channel, the normalized pressure field can be denoted by

$$
g\left(r_{s}, z_{s}, z_{k}\right) \approx \alpha \sum_{n} u_{n}\left(z_{s}\right) u_{n}\left(z_{k}\right) \frac{e^{i k_{n} r_{s}}}{\sqrt{k_{n} r_{s}}}
$$

where a constant density in the water column is assumed, and $\alpha$ is a scale factor so 
that the norm of the pressure field across the receiver array is unity. To express this signal field as a function of source location, $\left(r_{s}, z_{s}\right)$, we choose the coordinate origin at the receiver array, so the coordinate of the $k$ th sensor is $\left(0, z_{k}\right)$. In addition, we use real modal shape functions and assume the number of modes is fixed.

Effects of the environmental parameters $(\Psi)$ enter the signal field through the perturbation of the wavenumber, for example, adding $\delta k_{n}$ to $k_{n}$. Hence the ambiguity function for two sets of parameters, $\theta_{1}=\left(r_{s}, z_{s}, \Psi_{1}\right)$ and $\theta_{2}=\left(r_{s}+\delta r, z_{s}+\delta z, \Psi_{2}\right)$, is given by

$$
\begin{aligned}
\Gamma\left(f_{0}, \theta_{1}, \theta_{2}\right)= & \left|\alpha_{1} \alpha_{2}\right|^{2} \mid \sum_{k} \sum_{n} \sum_{m} \\
& \left.u_{n}\left(z_{s}\right) u_{n}\left(z_{k}\right) v_{m}\left(z_{s}+\delta z\right) v_{m}\left(z_{k}\right) \frac{e^{-i k_{n} r_{s}}}{\sqrt{k_{n} r_{s}}} \frac{e^{i\left(k_{m}+\delta k_{m}\right)\left(r_{s}+\delta r\right)}}{\sqrt{\left(k_{m}+\delta k_{m}\right)\left(r_{s}+\delta r\right)}}\right|^{2} .
\end{aligned}
$$

The general behavior of this ambiguity function is quite complicated. For the case with $\Psi_{1} \approx \Psi_{2}$, we have $u_{n}(z) \approx v_{n}(z)$ and $\delta k_{n} \ll k_{n}$. Expression in (4.2) can be simplified if the receiver array spans the entire water column so that the orthogonality of the propagation modes can be exploited, that is

$$
\sum_{k} \frac{1}{\rho\left(z_{k}\right)} u_{n}\left(z_{k}\right) u_{m}\left(z_{k}\right) \Delta d \approx \begin{cases}1, & n=m \\ 0, & n \neq m\end{cases}
$$

where $\Delta d$ is the sensor spacing. After some straightforward algebra,

$$
\begin{aligned}
\Gamma\left(f_{0}, \boldsymbol{\theta}_{1}, \boldsymbol{\theta}_{2}\right) \approx & \frac{\rho^{2}\left|\alpha_{1} \alpha_{2}\right|^{2}}{(\Delta d)^{2} r_{s}\left(r_{s}+\delta r\right)} \sum_{n}^{n_{\max }} \sum_{m}^{n_{\max }} \frac{u_{n}\left(z_{s}\right) u_{n}\left(z_{s}+\delta z\right)}{k_{n}} \frac{u_{m}\left(z_{s}\right) u_{m}\left(z_{s}+\delta z\right)}{k_{m}} \\
& \cos \left(\left(k_{n}-k_{m}\right) \delta r+\left(\delta k_{n}-\delta k_{m}\right) r_{s}+\left(\delta k_{n}-\delta k_{m}\right) \delta r\right)
\end{aligned}
$$

where $n_{\max }$ is again the number of propagation modes. Under this approximation, some interference pattern can be expected due to the $\cos (\cdot)$ term, but hardly predicted in an exact way. 
We now look at the ambiguity function for source range, depth, and environmental parameters, individually.

\subsubsection{Range-dependence}

Given $\theta_{1}=\left(r_{s}, z_{s}, \Psi\right)$ and $\theta_{2}=\left(r_{s}+\delta r, z_{s}, \Psi\right)$, and using (4.3), (4.2) becomes

$$
\Gamma\left(f_{0}, \delta r\right) \approx \frac{\rho^{2}\left|\alpha_{1} \alpha_{2}\right|^{2}}{(\Delta d)^{2} r_{s}\left(r_{s}+\delta r\right)} \sum_{n}^{n_{\max }} \sum_{m}^{n_{\max }} \frac{u_{n}^{2}\left(z_{s}\right)}{k_{n}} \frac{u_{m}^{2}\left(z_{s}\right)}{k_{m}} \cos \left(\left(k_{n}-k_{m}\right) \delta r\right) .
$$

We see the ambiguity comes from the term of $\cos \left(\left(k_{n}-k_{m}\right) \delta r\right)$, which is periodic with period of $2 \pi /\left(k_{n}-k_{m}\right)$. Interactions between different modes introduce some (often complicated) interference pattern, whose maxima correspond to sidelobe locations [68].

Consider source range estimation under the given environment in Fig. 4-1. In this example, the source depth is constant at $60 \mathrm{~m}$, and the source range varies from $3500 \mathrm{~m}$ to $4750 \mathrm{~m}$. Fig. 4-2 presents four ambiguity functions based on different sets of modes. We first notice that as the number of modes used increases, the mainlobe width decreases indicating a better resolution, while the number of sidelobes increases demonstrating a more complicated ambiguity structure. Second, adding more modes has little effect on the ambiguity function shown in Fig. 4-2(d). This suggests that the first ten modes be enough to represent the signal field in this example, which indeed include all the water-borne propagation modes.

\section{Array with full column spanning}

To study the effects of the array column spanning, we extend the previous sensor array using two approaches. The first one maintains the sensor spacing but adds 10 more sensors, spanning from $6 \mathrm{~m}$ to $196 \mathrm{~m}$. The second one still uses 16 sensors but increases the sensor spacing, spanning the water column from $1.5 \mathrm{~m}$ to $196.5 \mathrm{~m}$. Fig. 4-3 displays the ambiguity functions for both cases.

Compared to Fig. 4-2(d), the peak sidelobe level is about one dB lower. That 

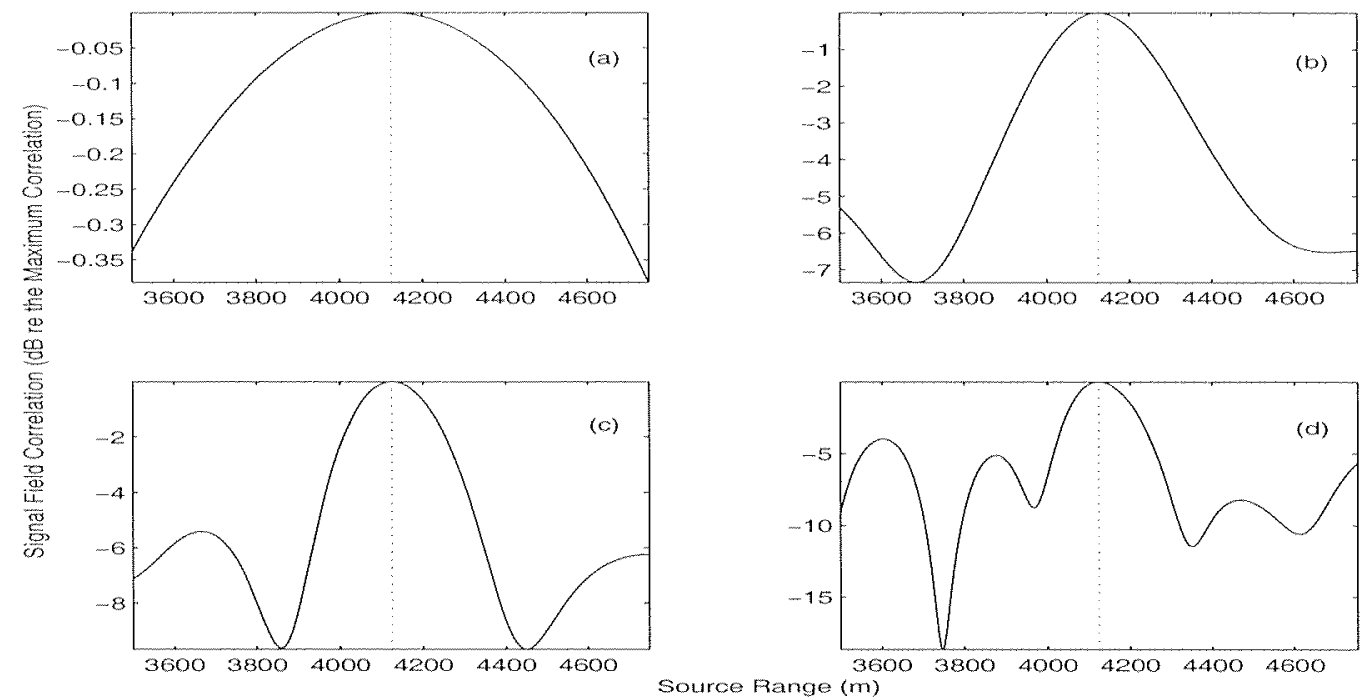

Figure 4-2: Signal ambiguity function for source range estimation. The number of modes used is (a) 2 ; (b) 6 ; (c) 8 ; (d) 10. The dotted line indicates the true source range position.

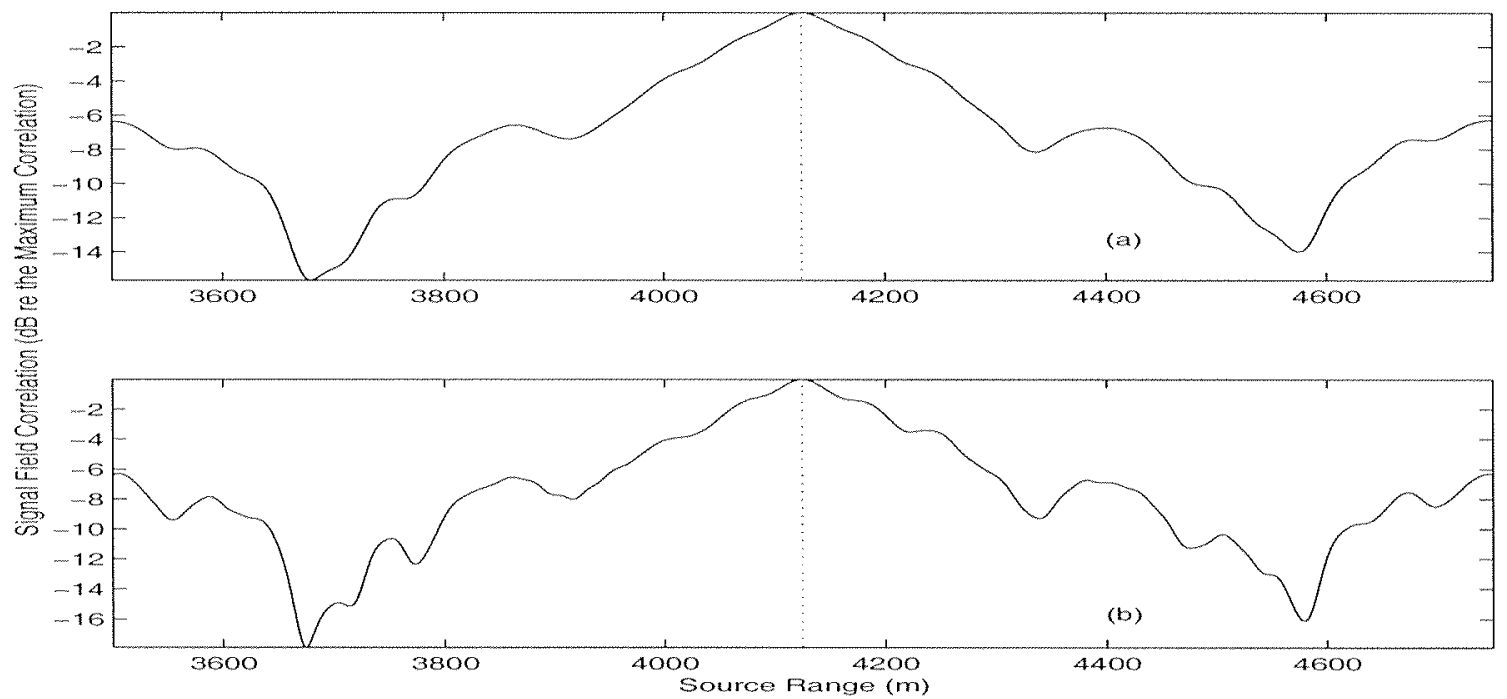

Figure 4-3: Signal ambiguity function for source range estimation using: (a) 26 sensors; (b) 16 sensors. In both cases, the array aperture spans the full water column. The dotted line indicates the true source range position. 
shows increasing the array column spanning helps to resolve the signal field. Besides, although the sparse array gives a little more ambiguous structure, ambiguity functions are very similar for both extensions. This is because the propagation signal arrivals concentrate on the broadside direction rather than the endfire direction, so the sparse array still well samples the field.

\section{Frequency-dependence}

The interference pattern in (4.5) has been exploited in relation to frequency to find estimates of the source range [68].

Note from (4.5) that interference maxima occur at

$$
\delta r=l \frac{2 \pi}{k_{n}-k_{m}}, \quad l=0, \pm 1, \pm 2, \ldots
$$

In an ideal waveguide, $k_{n}-k_{m}$ for low-order modes can be approximated by [68]:

$$
k_{n}-k_{m} \approx \frac{\left(n^{2}-m^{2}\right) c \pi^{2}}{\omega D^{2}}
$$

where $\mathrm{c}$ is the sound speed of the medium, D is the depth of the waveguide, and $\omega$ is the angular frequency. Therefore, for an ideal shallow water waveguide, the sidelobe distance is related to frequency by

$$
\delta r=l \frac{2 D^{2}}{\left(n^{2}-m^{2}\right) c \pi} \omega, \quad l=0, \pm 1, \pm 2, \ldots, n, m=1,2, \ldots
$$

Similar expressions are also available for some more general waveguides but with different powers of $\delta r$.

One may find the source range by looking at the convergence of (4.8) with frequency. On the other hand, (4.8) gives an approximation to the frequency-dependence of the sidelobe trajectories. Fig. 2 of Ref. [68] shows such an example for the same environment in Fig. 4-1. Obviously, this approximation is good if the low-order modes dominate the field. 


\subsubsection{Depth-dependence}

For $\boldsymbol{\theta}_{1}=\left(r_{s}, z_{s}, \Psi\right)$ and $\boldsymbol{\theta}_{2}=\left(r_{s}, z_{s}+\delta z, \Psi\right)$, using (4.3), the ambiguity function can be simplified by

$$
\Gamma\left(f_{0}, \delta z\right) \approx \frac{\rho^{2}\left|\alpha_{1} \alpha_{2}\right|^{2}}{(\Delta d)^{2} r_{s}^{2}} \sum_{n}^{n_{\max }} \sum_{m}^{n_{\max }} \frac{u_{n}\left(z_{s}\right) u_{n}\left(z_{s}+\delta z\right)}{k_{n}} \frac{u_{m}\left(z_{s}\right) u_{m}\left(z_{s}+\delta z\right)}{k_{m}}
$$

Obviously, ambiguities in source depth estimation are attributed to the modal depth eigenfunction, $u_{n}(z)$. This modal shape function is often highly oscillatory, and the mode order $n$ indicates the number of nodes associated with the mode. Although in general there is no closed-form expression available for the modal shape function, the WKB approximation suggests that it be a function of the modal vertical wavenumber, $k_{z n}(z)$, and may be represented as a sum of $\exp \left( \pm i k_{z n} z\right)$ terms [68]. Therefore, we would expect the depth ambiguity function contains some $\cos \left(\left(k_{z n}-k_{z m}\right) \delta z\right)$ terms.

Using the same environment in Fig. 4-1 for source depth estimation, we compute four ambiguity functions based on different sets of modes, shown in Fig. 4-4. The source range is fixed at $4125 \mathrm{~m}$ and the source depth varies from $30 \mathrm{~m}$ to $150 \mathrm{~m}$. Similar to the range ambiguity function, with more modes used, the depth estimation resolution is improved but the sidelobe ambiguity structure is more complicated. Again, the ambiguity function of the entire signal field is well specified using the first ten modes. In addition, some decreased sidelobe levels are observed in comparison to the range estimation case. This could be due to the nulls in modal shape function.

If, on the other hand, the true source is located at one of those nulls, the decreased signal power would lead to some performance degradation. It is worth noting that since the interference pattern of $\Gamma\left(f_{0}, \delta z\right)$ depends on $z_{s}$, the ambiguity property is expected to vary along the depth. 

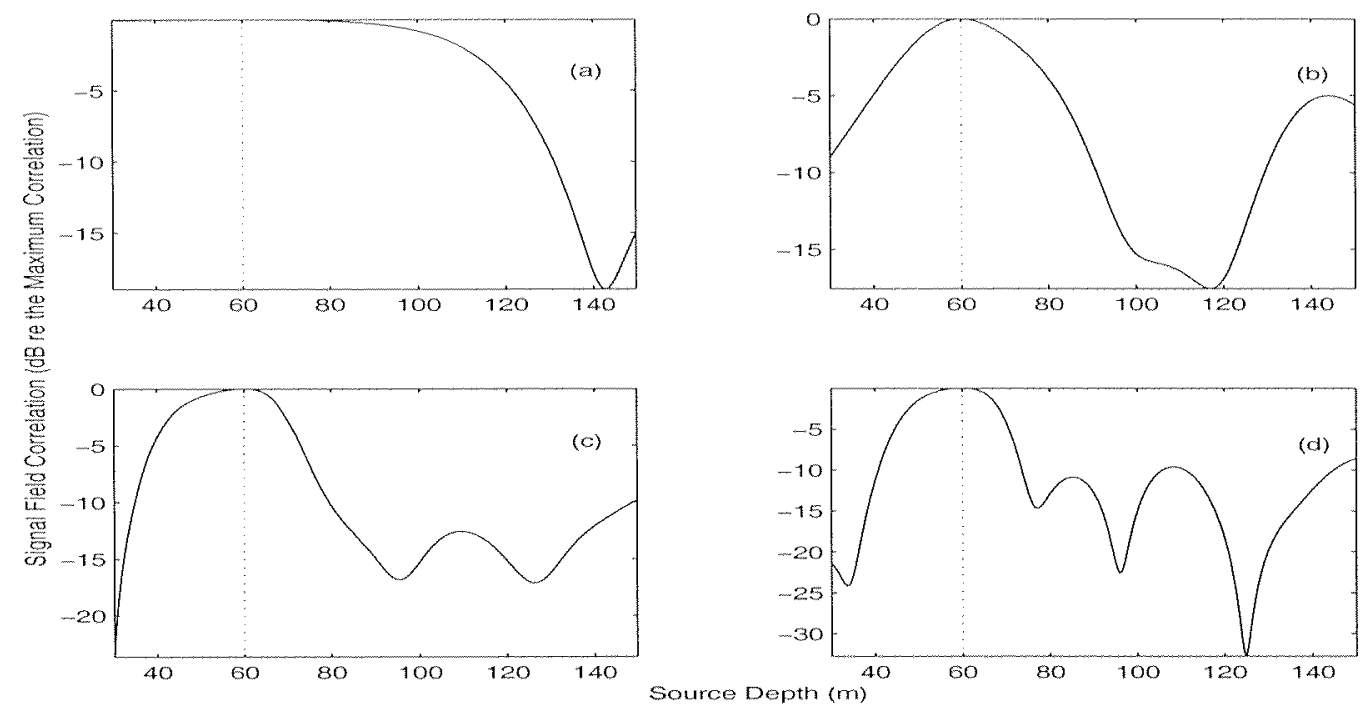

Figure 4-4: Signal ambiguity function for source depth estimation. The number of modes used is (a) 2 ; (b) 5 ; (c) 7 ; (d) 10. The dotted line indicates the true source depth position.

\subsubsection{Environmental-dependence}

In this case, we have $\boldsymbol{\theta}_{1}=\left(r_{s}, z_{s}, \Psi_{1}\right)$ and $\boldsymbol{\theta}_{2}=\left(r_{s}, z_{s}, \Psi_{2}\right)$, so the ambiguity function is

$$
\begin{aligned}
\Gamma\left(f_{0}, \Psi_{2}-\Psi_{1}\right)= & \left|\alpha_{1} \alpha_{2}\right|^{2} \mid \sum_{k} \sum_{n} \sum_{m} \\
& \left.u_{n}\left(z_{s}\right) u_{n}\left(z_{k}\right) v_{m}\left(z_{s}\right) v_{m}\left(z_{k}\right) \frac{e^{i\left(k_{m}-k_{n}\right) r_{s}+i \delta k_{m} r_{s}}}{\sqrt{k_{n} r_{s}} \sqrt{\left(k_{m}+\delta k_{m}\right) r_{s}}}\right|^{2} .
\end{aligned}
$$

As mentioned before, the environmental parameter set determines modal eigenvalues (wavenumbers) as well as eigenfunctions. Accordingly, environmental variations introduce wavenumber variations, resulting in some $\exp \left(i \delta k_{m} r_{s}\right)$ terms in $(4.10)$. Clearly, the ambiguity in environmental parameter estimation is due to those modal wavenumber variations, which are amplified by source range, particularly at longrange.

\footnotetext{
${ }^{1}$ Even though the scanning interval of $\Psi$ is chosen such that $u_{n}(z) \approx v_{n}(z)$ and $\delta k_{n} \ll k_{n},(4.3)$ is
} 


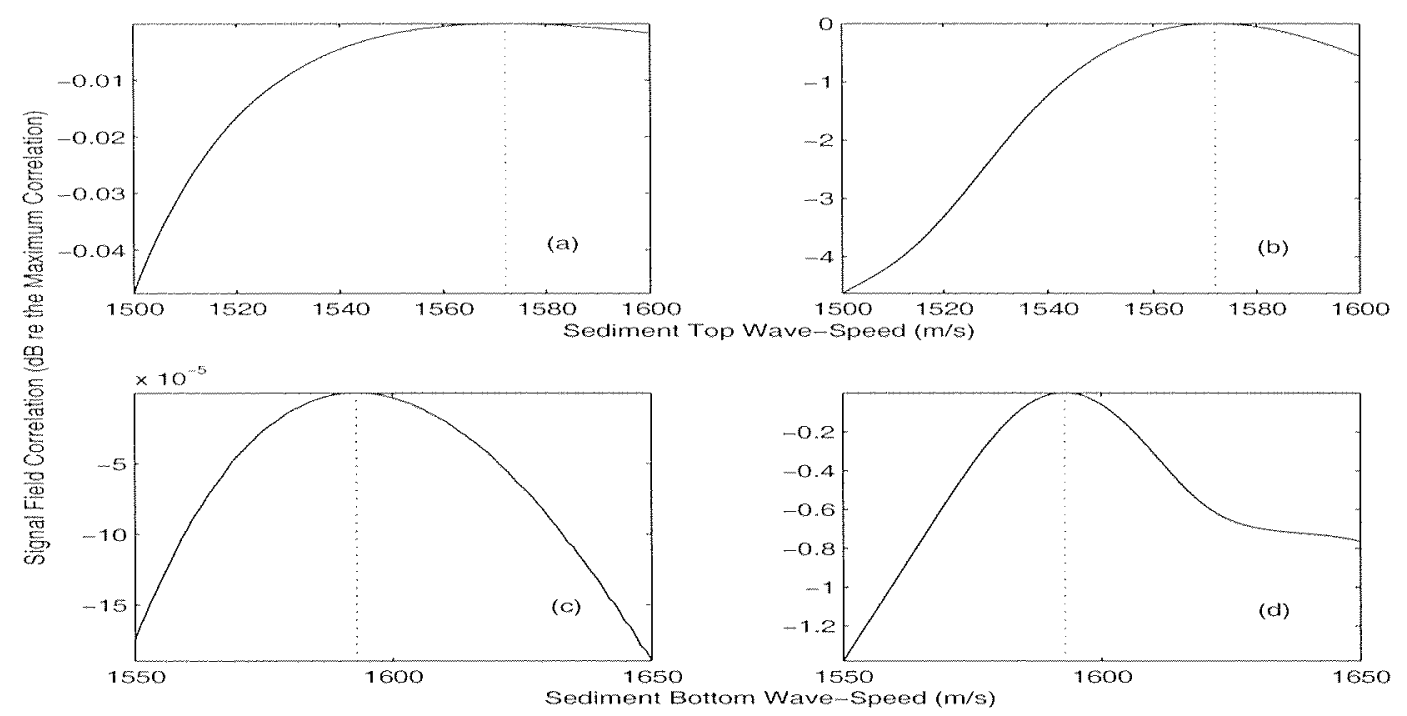

Figure 4-5: Signal ambiguity function for sediment wave-speed estimation. The number of modes used is (a) 2; (b) 14 for top wave-speed estimation; (c) 2; (d) 21 for bottom wave-speed estimation. The dotted line indicates the true wave-speed position.

An example for sediment wave-speed estimation is shown in Fig. 4-5. To describe the sediment-dependent field ambiguity, some higher-order modes have to be included (14 modes for sediment top wave-speed; 21 modes for bottom wave-speed). Because the wavenumber variations are quite small, the ambiguity structure is much smoother compared to the range or depth estimation case. This smooth mainlobe structure may support the linear perturbation approach for environmental parameter inversion [57], although the resolution can be quite limited. Similar ambiguity behaviors are observed for sediment attenuation and density.

In contrast, some geometry parameters may demonstrate significant ambiguities. For example, the sediment thickness often introduces time-delays to those modes propagating in the sediment and refracted (or reflected) back to the water column. As discussed in Section 2.1.2, the time-delay effect can be represented by wavenumber variation. Thus, if those (sediment-borne) modes are not important in the signal field, we can expect some sidelobe peaks, due to those high-order modes, overlying a wide

no longer useful for bottom parameters. This is because the bottom information is mainly contained in the high-order (leaky) modes, which do not, satisfy (4.3) at the receiver array. 


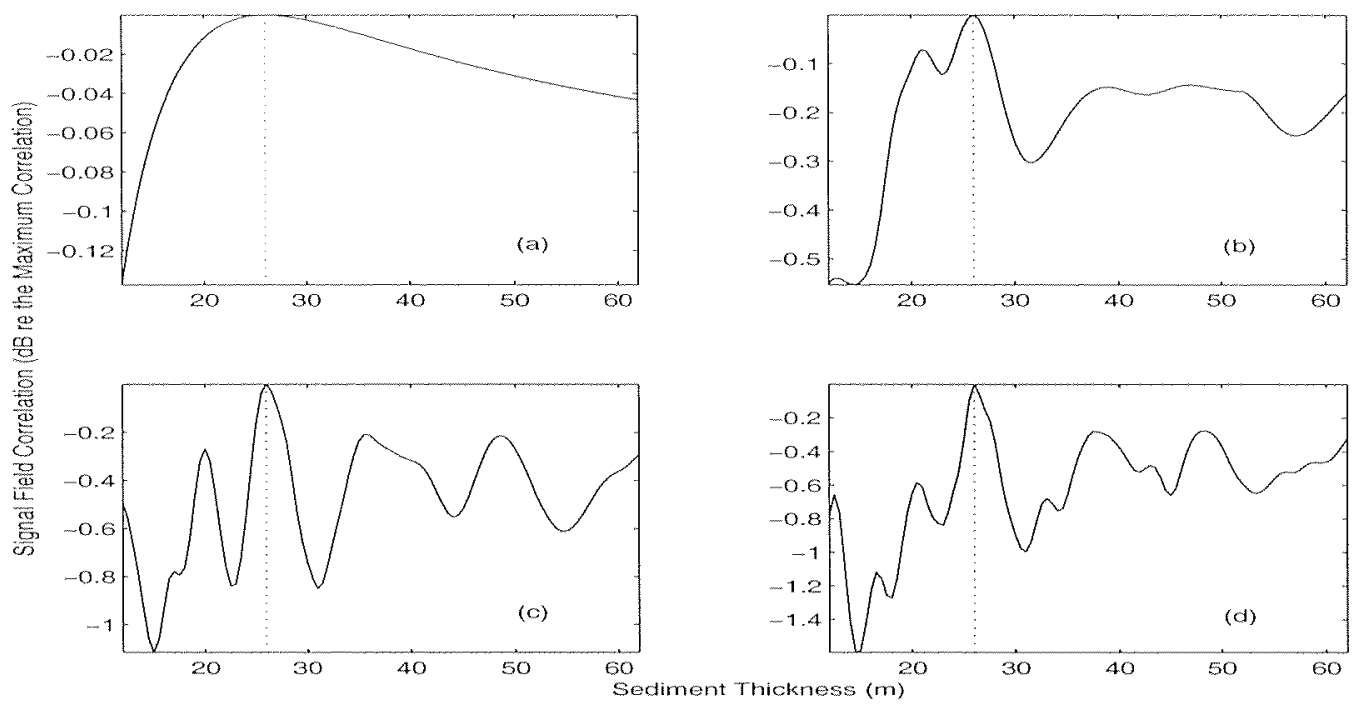

Figure 4-6: Signal ambiguity function for sediment thickness estimation. The number of modes used is (a) 9; (b) 13; (c) 16; (d) 21. The dotted line indicates the true thickness position.

mainlobe due to the low-order modes. Otherwise, some complicated sidelobe structure is expected. Fig. 4-6 gives such an example for the thickness of the sediment layer under the environment in Fig. 4-1.

In this example, because the wave-speed at the top of the mudstone layer is much higher than that at the bottom of the sediment layer, the mudstone layer acts as a rigid basement. When the thickness of the sediment layer varies from $12 \mathrm{~m}$ to 62 $\mathrm{m}$, those sediment-borne modes play a significant role in the wave field. As shown in Fig. 4-6, when only the first nine modes are used, a wide mainlobe is observed. As more and more modes are incorporated, the field shows highly-oscillatory sidelobe behaviors, although we can still see an envelope of the mainlobe. A total of twenty-one modes is required to represent the field ambiguity.

\subsection{Ambiguity Mainlobe: Parameter Coupling}

Investigation for individual parameters tells us that the field ambiguity arises from interferences between modes associated with different parameters, which produce some (quasi-)periodic function terms of range/depth/wavenumber variations. Generaliza- 
tion of the above analysis to multi-dimensional parameter space is much more complicated. This is mainly due to complicated parameter-dependences of the field, which sometimes demonstrate strong inhomogeneity and mutual coupling. To visualize a multi-dimensional ambiguity function, one often resorts to its two-dimensional slices. In this section, we briefly discuss some two-dimensional cases, particularly how the parameter coupling shapes the ambiguity function.

As discussed in Section 3.1, the parameter coupling describes how the uncertainty in estimation of one parameter correlates with the uncertainty in estimation of another parameter. This description is valid only at high SNR levels and thus is closely related to local field sensitivities to parameters. In other words, the parameter coupling describes how a change of the signal field associated with one particular parameter perturbation is related to that associated with another parameter perturbation. Obviously, the shape of the ambiguity mainlobe gives a perfect indication of the parameter coupling. Elongation of the mainlobe along the horizontal or vertical direction corresponds to weak parameter coupling, while elongation along the diagonal direction corresponds to strong parameter coupling.

Let us first look at the source range-depth estimation problem. Since the environment is perfectly known, the wavenumber variation in (4.4) is eliminated, and

$$
\begin{aligned}
\Gamma\left(f_{0}, \delta z, \delta r\right) \approx & \frac{\rho^{2}\left|\alpha_{1} \alpha_{2}\right|^{2}}{(\Delta d)^{2} r_{s}\left(r_{s}+\delta r\right)} \\
& \sum_{n}^{n_{\max }} \sum_{m}^{n_{\max }} \frac{u_{n}\left(z_{s}\right) u_{n}\left(z_{s}+\delta z\right)}{k_{n}} \frac{u_{m}\left(z_{s}\right) u_{m}\left(z_{s}+\delta z\right)}{k_{m}} \cos \left(\left(k_{n}-k_{m}\right) \delta r\right) .
\end{aligned}
$$

We see that $\delta r$ and $\delta z$ enter the ambiguity function separately, and $\cos \left(\left(k_{n}-k_{m}\right) \delta r\right)$ and $u_{n}\left(z_{s}+\delta z\right)$ are two different (quasi-)periodic functions. Accordingly, we would expect a weak coupling between source range and depth. This is observed from Figs. 1-1 and 4-7. Similar to many other examples of matched-field processing $[9,17]$, there is little mainlobe elongation along a diagonal direction.

In fact, a strong parameter coupling can often exist between two environmental 


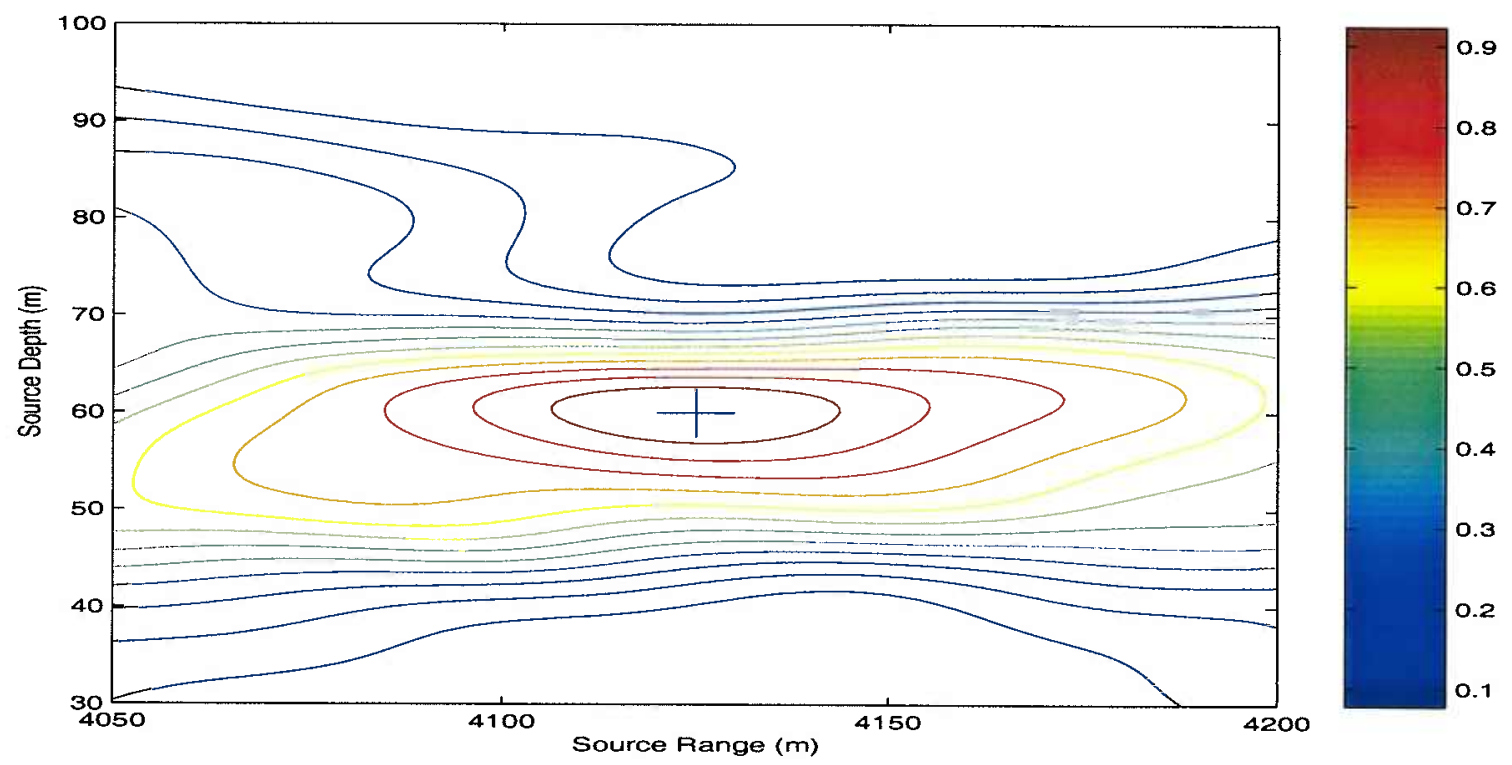

Figure 4-7: Ambiguity mainlobe contour for source range and depth estimation. The cross sign indicates the true source position.

parameters, when they act in a consistent way such that the wavenumber variations of modes (usually a subset of modes) are maintained. For example, the sediment top and bottom wave-speeds are strongly coupled under the previously-defined environment, as shown in Fig. 4-8(b). For this environment (cf. Fig. 4-1), the wave field is actually determined by reflections at the water-sediment interface as well as reflections at the interface between the sediment layer and the mudstone layer. A positive perturbation of the sediment top wave-speed increases the effective critical angle (measured from the horizontal line), and thus decreases the modal penetration into the sediment layer. However, this effect is canceled by a negative perturbation of the bottom wave-speed, which intends to increase the effective critical angle between the sediment layer and the mudstone layer and thus decrease the modal penetration into the mudstone layer. As a balance, the sediment-borne modes are about maintained. Accordingly, when only the water-borne modes are used, there is just a little coupling between these two wave-speeds (cf. Fig. 4-8(a); the slope of the mainlobe elongation is about -0.25); when the sediment-borne modes are incorporated, we can see a strong (negative) coupling between them (the slope is about -0.95).

Note that for the environment given in Fig. 4-1, because the reflections at both 


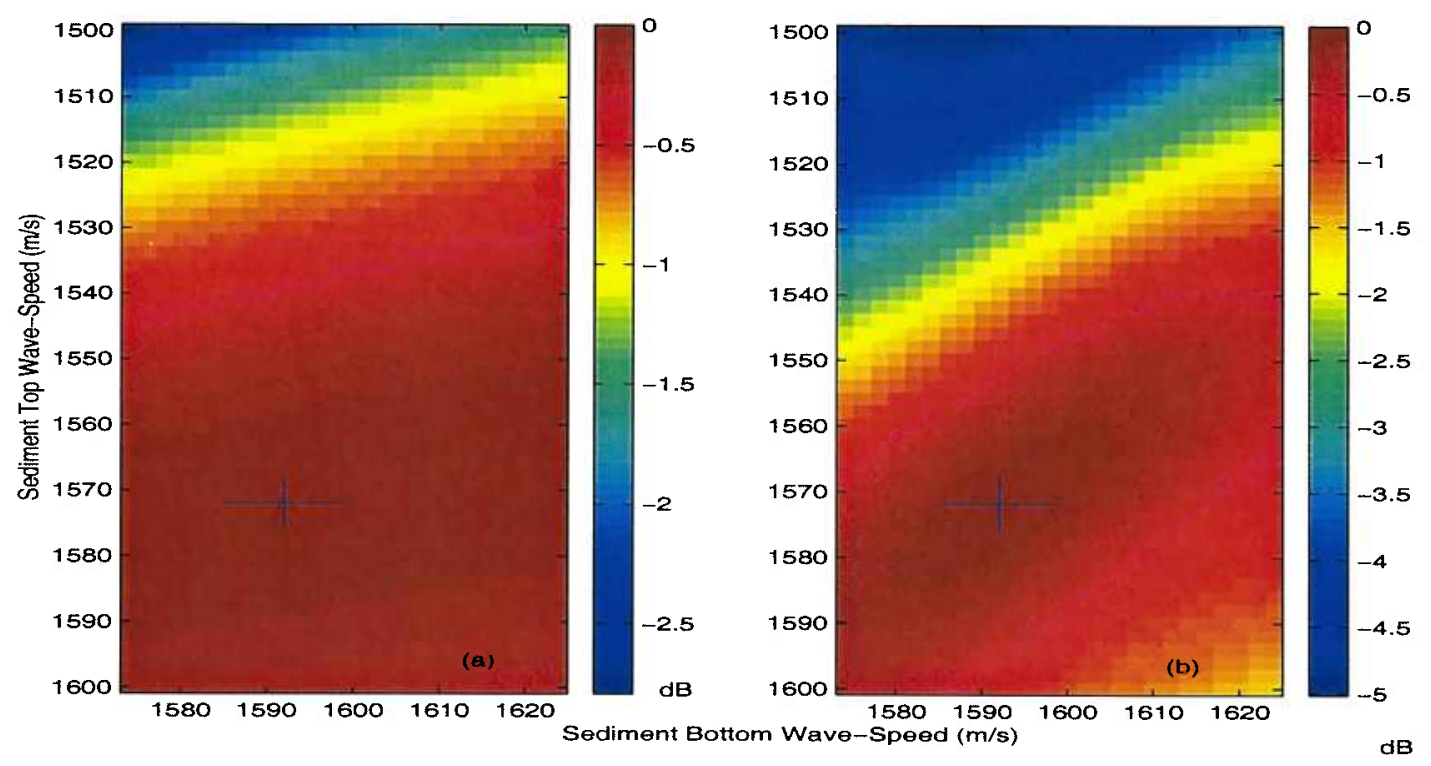

Figure 4-8: Signal ambiguity function for sediment top and bottom wave-speed estimation. The number of modes used is: (a) 8; (b) 22. The cross sign indicates the true parameter position.

interfaces dominate the wave field, the refraction and then the wave-speed gradient in the sediment layer are less influential. Consequently, the coupling of the sediment thickness with either the top wave-speed or the bottom wave-speed is small. The situation is different when we replace the mudstone layer by a basement with wavespeed equal to the bottom one of the sediment layer, as discussed in Ref. [63]. In this case, there is no reflection at the sediment-basement interface. Instead, part of the sound penetration in the sediment layer is refracted back to the water column, which is determined by the wave-speed gradient. The wave-speed gradient is maintained by simultaneously increasing the sediment thickness and bottom wave-speed. Thus, a strong coupling between these two parameters is observed.

Coupling of the environmental parameters with the source location is through the variations of the wavenumbers. Because the wavenumber variation, $\delta k_{n}$, and the source position variation, $\delta r$ or $\delta z$, enter the ambiguity function in a very complicated way, coupling analysis is often analytically intractable. It is true that there could be no coupling if the wave field has little dependence on the chosen parameters, for example, some deeper bottom properties. Nonetheless, large wavenumber variations 


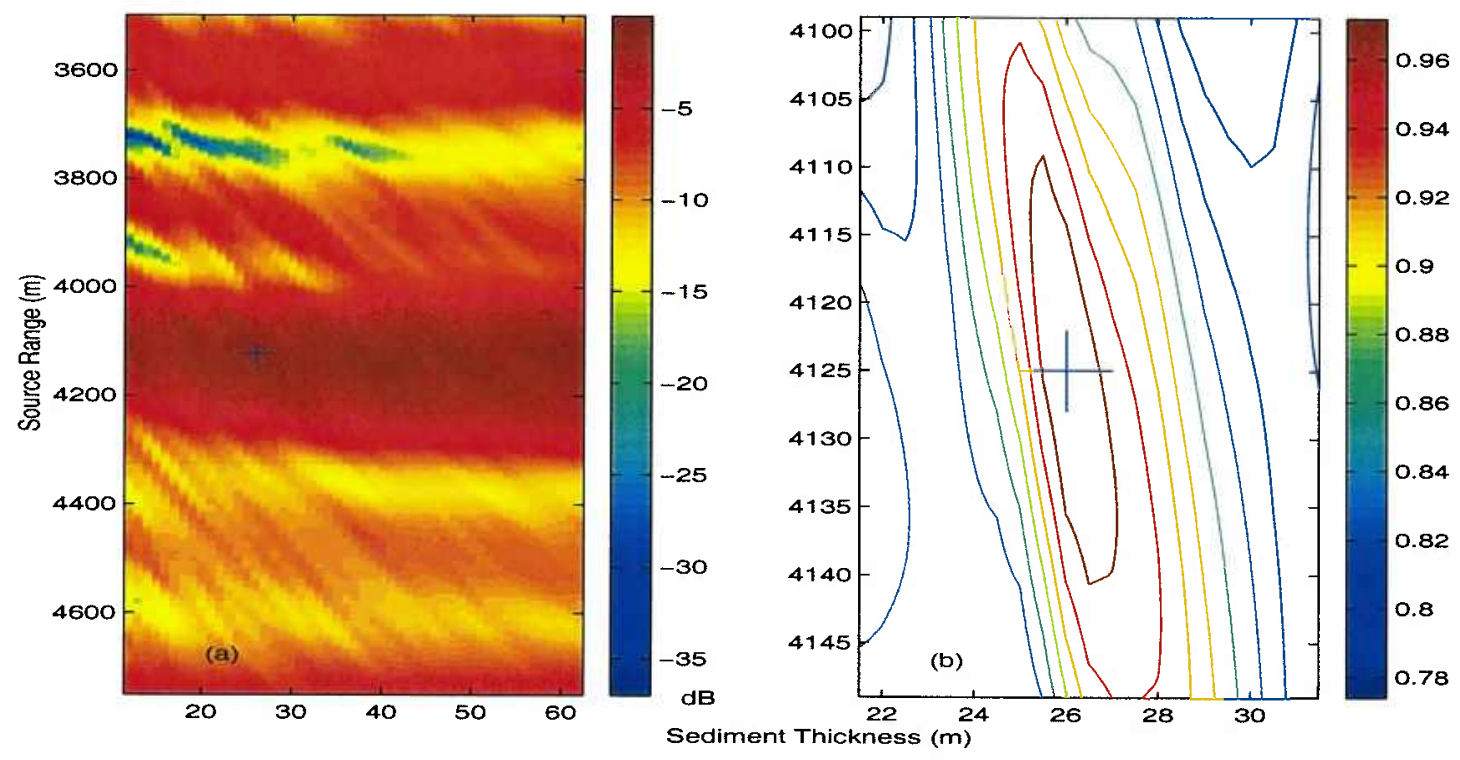

Figure 4-9: Signal ambiguity function for source range and sediment thickness estimation: (a) ambiguity function; (b) mainlobe contours. The cross sign indicates the true parameter position.

do not necessarily lead to a strong coupling between any source parameter and the environmental parameter introducing those large wavenumber variations.

For example, we know that the wave field is very sensitive to the sediment thickness, as shown in Fig. 4-6. However, from Fig. 4-9, the mainlobe elongation is close to vertical $^{2}$ (the slope is about 25), indicating a weak coupling between the source range and the sediment thickness. This is because the phase variation introduced by the thickness is much faster than that by the range due to larger vertical wavenumbers of the sediment-borne modes.

It is interesting to note that, given the environment in Fig. 4-1, the source range estimation is strongly coupled to both the sediment top and bottom wave-speeds, as shown in Fig. 4-10 (both slopes are about -1 second). This is again determined by the modal wavenumber variations of those sediment-borne modes, which is comparable to the source range variation. Hence, to obtain a good estimate of the source range, knowledge about the sediment wave-speed is important.

To avoid misleading the reader, we have to point out that one should be very

\footnotetext{
${ }^{2}$ To make this observation clear, one must use some (standard) comparable units for parameters and scale the ambiguity plot properly.
} 

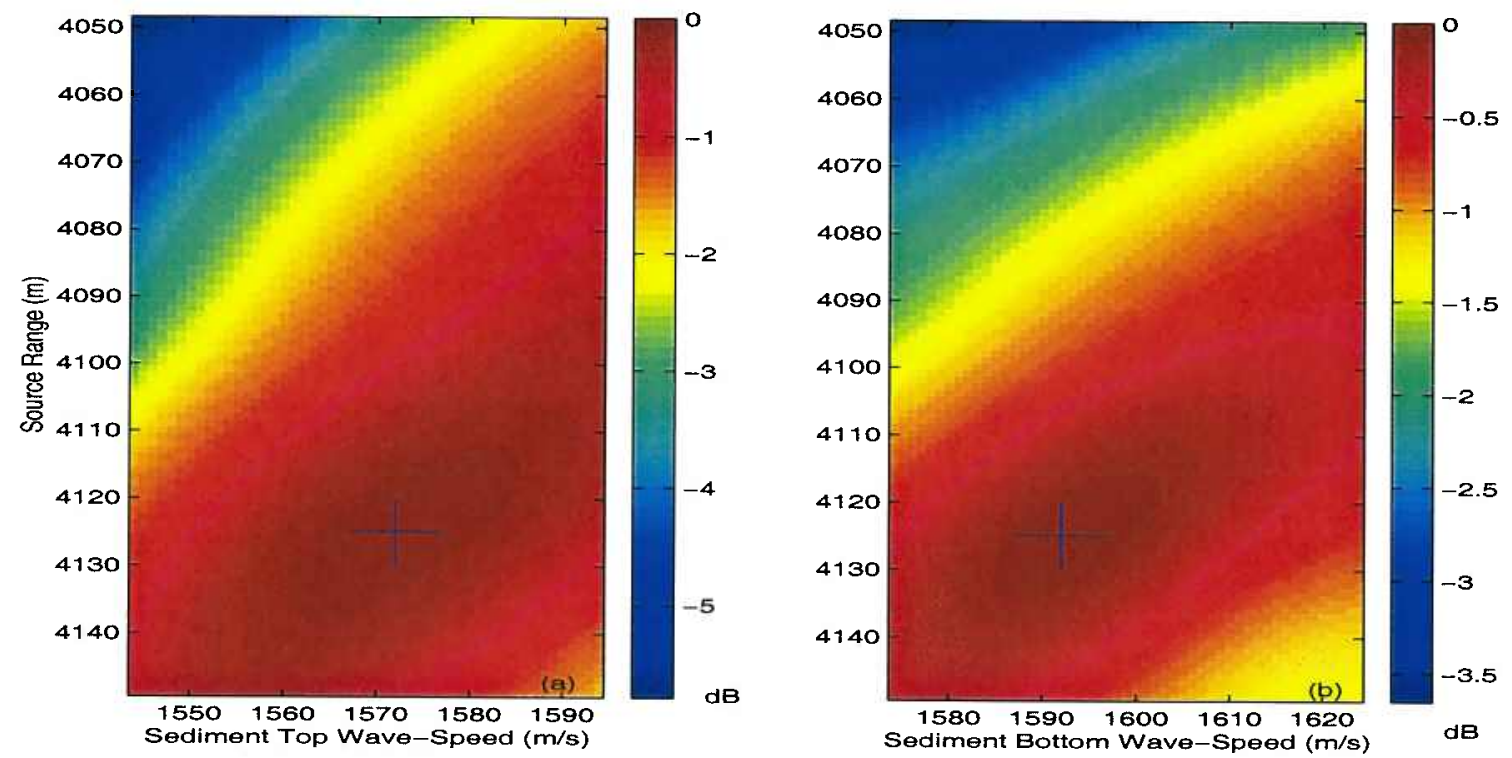

Figure 4-10: Signal ambiguity function for source range and sediment wave-speed estimation: (a) top wave-speed; (b) bottom wave-speed. The cross sign indicates the true parameter position.

careful before drawing a general conclusion for the coupling between two parameters. This is because the parameter coupling often depends on a specific environment as well as specific parameter values, and the ocean environment often shows strong coupling variability within the parameter space.

Generalization of the coupling description to sidelobe ambiguity shape should be possible, but will be more difficult for physical interpretation.

\subsection{Performance Bound and Ambiguity}

In previous sections, we have seen that the signal field correlation can be described in terms of the mainlobe and sidelobe behaviors. For the purpose of performance analysis, it is desired to connect the multi-modal correlation structure with the performance in parameter estimation. This can be done by analyzing the performance bound in terms of the signal field correlation, as given in this section. Indeed, whether and how well a performance bound captures the sidelobe behaviors determine its capability to predict the threshold SNR as well as performance in the threshold region. An alternative approach is also developed in the context of the maximum likelihood 
estimate, which is elaborated in the next section.

\subsubsection{Bayesian Cramer-Rao Bound}

Parameter estimation resolution is well predicted by the CRB given that the global peak of the ambiguity function has been correctly located. This is often the case at high SNR levels, and the local information matrix in (2.17) can be further simplified.

Note that for a white noise process, we have $S_{n}(f)=S_{n}(f) \mathrm{I}$. Under this assumption, we can define a signal-to-noise ratio term by

$$
\operatorname{SNR}(f)=\frac{S_{b}(f)\|\mathbf{G}(f, \theta)\|^{2}}{S_{n}(f)} .
$$

If we ignore the parameter-dependence of the norm of the Green's function, then by factoring the $S_{n}(f)$ term, the local information matrix at high SNR becomes

$$
\left[\mathrm{J}_{D}(\boldsymbol{\theta})\right]_{i j}=\sum_{m=1}^{M} S N R\left(f_{m}\right)\left[\bar{l}_{i, j}\left(f_{m}, \boldsymbol{\theta}\right)-\bar{l}_{i}\left(f_{m}, \boldsymbol{\theta}\right) \bar{l}_{j}^{\dagger}\left(f_{m}, \boldsymbol{\theta}\right)\right]
$$

where

$$
\begin{aligned}
\bar{l}_{i}\left(f_{m}, \boldsymbol{\theta}\right) & =\mathbf{g}^{\dagger}\left(f_{m}, \boldsymbol{\theta}\right)\left(\frac{\partial}{\partial \theta_{i}} \mathbf{g}\left(f_{m}, \boldsymbol{\theta}\right)\right) \\
\bar{l}_{i, j}\left(f_{m}, \boldsymbol{\theta}\right) & =\left(\frac{\partial}{\partial \theta_{i}} \mathbf{g}^{\dagger}\left(f_{m}, \boldsymbol{\theta}\right)\right)\left(\frac{\partial}{\partial \theta_{j}} \mathbf{g}\left(f_{m}, \boldsymbol{\theta}\right)\right) .
\end{aligned}
$$

Now it is obvious that the local information matrix is determined (through (4.14) and (4.15)) from the shape of the ambiguity mainlobe, i.e., the slope and curvature at the peak point. This connects the ambiguity mainlobe with the Cramer-Rao resolution matrix and thus the parameter coupling. A typical way to illustrate the coupling is computing the eigenvectors of the resolution matrix and then mapping them to the parameter space. The angles of the eigenvectors with respect to the parameter axes represent the coupling [63]. One can also use a correlation coefficient, which is the error covariance (associated with two chosen parameters) normalized to the individual resolutions [27]. Clearly, this coupling measure falls between -1 and 
+1 .

One must be careful when interpreting a parameter coupling predicted by the Bayesian CRB. It is the averaged parameter coupling over the parameter space, and the local coupling could be highly inhomogeneous in both amplitude and direction within this space.

\subsubsection{Weiss-Weinstein Bound}

The WWB can be used to predict the threshold SNR as well as the performance in the threshold region. To study the bound's behavior, we consider a simplified case with a single test point $h$, a single parameter $\theta$, and a single snapshot centered at $f_{0}$. For a uniform distribution of $\theta$ over $[-\tau, \tau]$ and $\tau>h>0$, the mean square error is bounded by

$$
\epsilon^{2} \geq \max _{h} \frac{\frac{h^{2}}{4 \tau}\left(\int_{-\tau}^{\tau-h} d \theta \frac{1}{\eta\left(f_{0}, \theta, h\right)}\right)^{2}}{2 \tau-h-\int_{-\tau}^{\tau-2 h} d \theta \frac{1}{\eta\left(f_{0}, \theta, 2 h\right)}}
$$

where $\eta\left(f_{0}, \theta, h\right)$ is defined according to $(3.43)$.

It can be shown (cf. Appendix A) that $\eta\left(f_{0}, \theta, h\right) \geq 1$ and equality holds if and only if $\left|C\left(f_{0}, \theta, h\right)\right|^{2}=1$ and $A\left(f_{0}, \theta\right)=B\left(f_{0}, \theta, h\right)$, which indicates $\theta+h$ is a completely ambiguous point of $\theta$. From (4.16), a small $\eta\left(f_{0}, \theta, h\right)(\approx 1)$ is desired for maximizing the bound. Accordingly, the optimum choice of $h$ is related to the ambiguity points.

Recall that $A\left(f_{0}, \theta\right)$ and $B\left(f_{0}, \theta, h\right)$ are SNR-related terms and $\left|C\left(f_{0}, \theta, h\right)\right|^{2}$ is actually the field correlation. In a limited parameter interval, we often have local homogeneity of $\|\mathbf{G}\|$, i.e., $\left\|\mathbf{G}\left(f_{0}, \theta+h\right)\right\| \approx\left\|\mathbf{G}\left(f_{0}, \theta\right)\right\|$, and thus $A\left(f_{0}, \theta\right) \approx$ $B\left(f_{0}, \theta, h\right)$. Hence, the ambiguity behavior acts on the bound's evaluation via the variation of $C\left(f_{0}, \theta, h\right)$. In this case,

$$
\eta\left(f_{0}, \theta, h\right) \approx 1+\frac{1}{4} \cdot \frac{A^{2}\left(f_{0}, \theta\right)}{1-A\left(f_{0}, \theta\right)} \cdot\left(1-\left|C\left(f_{0}, \theta, h\right)\right|^{2}\right) .
$$

We see that with $\theta$ as the true parameter and $\theta+h$ as the scanning parameter, the 
term of $\frac{1}{\eta\left(f_{0}, \theta, h\right)}$ in (4.16) has a similar "estimate-subtract" structure as the MVDR ambiguity output [9] (see also Appendix A.3.3). When $|C|^{2}$ equals unity, which is the case when scanning at the true parameter, $1 / \eta$ equals one. This corresponds to the lobe of the maximum at the true parameter. When $|C|^{2}$ equals zero, which is the case of no sidelobes,

$$
\frac{1}{\eta\left(f_{0}, \theta, h\right)}=\sigma_{n}^{2} \cdot \frac{4\left(S N R\left(f_{0}\right)+1\right)}{\left(S N R\left(f_{0}\right)+2\right)^{2}}
$$

Except at high SNR, $1 / \eta$ is usually larger than $\sigma_{n}^{2}$, the corresponding MVDR output when no sidelobes. This is reasonable since the MVDR minimizes the mean square response to noise field. When $|C|^{2}$ equals some value between zero and one, which occurs at a sidelobe or off the center of the mainlobe, this "estimate-subtract" structure leads to the sidelobe suppression. Specifically, to have

$$
\frac{\frac{1}{\eta\left(f_{0}, \theta, h\right)}}{\frac{1}{\eta\left(f_{0}, \theta, 0\right)}}=\frac{1}{1+\frac{1}{4} \cdot \frac{A^{2}\left(f_{0}, \theta\right)}{1-A\left(f_{0}, \theta\right)} \cdot\left(1-\left|C\left(f_{0}, \theta, h\right)\right|^{2}\right)} \geq \delta
$$

where $\delta$ is a pre-defined output level, we must have

$$
\left|C\left(f_{0}, \theta, h\right)\right|^{2} \geq 1-\frac{1-\delta}{\delta} \cdot \frac{4\left(1-A\left(f_{0}, \theta\right)\right)}{A^{2}\left(f_{0}, \theta\right)}
$$

In the high SNR region, $A \approx 1$, and thus $|C|^{2}$ must be very close to unity. A little departure of $|C|^{2}$ from unity results in a significant decrease of $1 / \eta$. In the low to intermediate SNR region, $|C|^{2}$ could be small and (4.19) still holds; therefore, the ambiguities often prevail in this SNR region.

Now it is obvious that sensitivity of $\eta\left(f_{0}, \theta, h\right)$ to variation of $\left|C\left(f_{0}, \theta, h\right)\right|$ decreases as the value of $A\left(f_{0}, \theta\right)\left(B\left(f_{0}, \theta, h\right)\right)$ and thus the SNR decrease. As a result, at high SNR, only a small $h$ achieves a small $\eta\left(f_{0}, \theta, h\right)$, while at intermediate SNR, some $h$ 's corresponding to sidelobe points achieve small $\eta\left(f_{0}, \theta, h\right)$. At low SNR, since the noise dominates the observation and the associated covariance matrix, it can be easily shown $\eta\left(f_{0}, \theta, h\right) \approx 1$ for all $\theta$ and $h$ within the parameter space. In this region, the right-hand-side of (4.16) is maximized by $h=\frac{2}{3} \tau$ and the maximum is $\frac{8}{27} \tau^{2}$, close to 
$\frac{1}{3} \tau^{2}$, variance of the uniform parameter distribution.

So the optimum choice of the test point indicates the major ambiguous parameter position, and they both vary as a function of the SNR. Accordingly, the threshold SNR can be defined as the SNR at which the optimum test point switches from a mainlobe point to a sidelobe point. For two sidelobes of the same level, the sidelobe with larger $h$ contributes more to the error bound since the numerator in (4.16) is amplified by $h^{2}$ while the denominator is linear with $h$. This can be understood because the error probability is solely determined by the signal field correlation (ambiguity) level and the SNR. In Sections 4.4 and 4.5, we have further discussions on the error probability as well as the threshold SNR.

\subsubsection{Ziv-Zakai Bound}

The ambiguity behavior is captured by the ZZB directly through the term of the minimum probability of error. As introduced in Appendix $\mathrm{C}$, the minimum probability of error is defined based on a two-point estimation problem. In other words, it relates to the probability that given one parameter point is the true one, the estimate is the other parameter point.

In evaluation of the ZZB (cf. (3.24)), for a given true parameter point $\boldsymbol{\theta}$, the minimum error probability needs to be solved for each parameter perturbation $\Delta$. From $(3.45),(3.48),(3.51)$ and $(3.52)$, we see that this error probability is determined by a signal ambiguity function term and a signal-to-noise ratio term. Let us restate the definition for the ambiguity function:

$$
C(f, \theta, \Delta)=\mathrm{g}^{\dagger}(f, \theta+\Delta) \mathrm{g}(f, \theta)
$$

Then for the single-frequency case, we can rewrite the error probability by

$$
P_{e}(\boldsymbol{\theta}+\Delta \mid \boldsymbol{\theta})=\frac{\sum_{k=0}^{L-1}\left(\begin{array}{c}
2 L-1 \\
k
\end{array}\right) \cdot\left(\lambda_{R}\right)^{k}}{\left(1+\lambda_{R}\right)^{2 L-1}}
$$


where

$$
\begin{aligned}
\lambda_{R} & =-\frac{\lambda_{1}}{\lambda_{2}} \\
& =\frac{1+\sqrt{1+\frac{4}{S N R\left(f_{0}\right)}\left(1+\frac{1}{S N R\left(f_{0}\right)}\right) \cdot \frac{1}{1-|C(f, \theta, \Delta)|^{2}}}}{-1+\sqrt{1+\frac{4}{S N R\left(f_{0}\right)}\left(1+\frac{1}{S N R\left(f_{0}\right)}\right) \cdot \frac{1}{1-|C(f, \theta, \Delta)|^{2}}}} .
\end{aligned}
$$

It can be easily verified that $\lambda_{R}>1$ and for $\lambda_{R}>0$,

$$
\frac{\partial}{\partial \lambda_{R}} P_{e}(\boldsymbol{\theta}+\Delta \mid \boldsymbol{\theta})<0
$$

Hence, the error probability decreases as $\lambda_{R}$ increases, either by increasing the SNR or decreasing the field correlation. Accordingly, for a fixed SNR level, the error probability is high when the field correlation associated with two chosen parameter points is high. For a fixed pair of parameter points (and thus fixed field correlation), the error probability increases as the SNR decreases. We also notice that there is an SNR-square dependence but a $|C|^{2}$-linear dependence of $\lambda_{R}$. Therefore, for two fixed field correlation levels, the difference between the associated error probabilities decreases as the SNR decreases.

Back to the bound's behavior, compared to sidelobe points, the field at a mainlobe point is usually more correlated with the field at the true parameter point, and thus the resulting error probability is higher. However, the differences decrease as the SNR decreases. On the other hand, in the bound's evaluation, the error probability is amplified by the parameter perturbation, which is often much larger for sidelobe points. As a result, at some SNR level, the sidelobe contribution is beyond the mainlobe contribution, demonstrating the threshold behavior. At very low SNR, $P_{e}(\boldsymbol{\theta}+\boldsymbol{\Delta} \mid \boldsymbol{\theta}) \approx 1 / 2$ for all $\boldsymbol{\theta}$ and $\boldsymbol{\Delta}$, then the ZZB is determined by the a priori parameter information. For a uniform parameter distribution over $[-\tau, \tau]$, the bound goes to $\tau^{2} / 3$.

Now we see some connections between the ZZB and the WWB. First, the error probability of the $\mathrm{ZZB}, P_{e}(\boldsymbol{\theta}, \boldsymbol{\theta}+\boldsymbol{\Delta})$, plays a similar role as the WWB ambiguity 
output, $1 / \eta\left(f_{m}, \boldsymbol{\theta}, \mathbf{h}\right)$. Second, the parameter perturbation of the ZZB, $\boldsymbol{\Delta}$, acts similarly as the test point of the WWB, $\mathbf{h}$, so that the ambiguity-related term $\left(P_{e}\right.$ or $1 / \eta)$ is amplified by the distance between the scanning parameter point and the true parameter point.

In spite of that, we would expect a tighter performance prediction around the threshold region given by the ZZB if the error probability is accurately solved. In evaluation of the ZZB, the parameter perturbations are chosen to cover all the mainlobe and sidelobe points, so the sidelobe contribution to the mean square error is included even though it is still below the mainlobe contribution. In contrast, the single-test point WWB captures the sidelobe contribution only when it is beyond the mainlobe contribution. Even though multiple test points are used, the number of test points is often limited due to some numerical concerns (e.g., inversion of the Q matrix in $(3.18))$.

Finally, we notice that for vector parameter estimation, evaluation of the ZZB requires a maximization of the minimum error probability with respect to the vector parameter perturbation $\Delta$ under the constraint that the projection of $\Delta$ onto the dimension of the chosen parameter is a constant (cf. (3.24)), $\delta$. This corresponds to locating the maximum field correlation level for a fixed distance, $\delta$, to the true parameter point along the dimension of the chosen parameter. Clearly, this field correlation peak does not necessarily occur with other parameter values equal to those at the true parameter point.

For example, as shown in Fig. 1-1, for a fixed distance to the true source position along the dimension of source range (corresponding to an arbitrary range ambiguity point), the maximum field correlation often occurs at a source depth different from that at the true source position. The resulting ambiguity plot along the dimension of source range is actually a projection of the two-dimensional ambiguity surface onto the range dimension by choosing the maximum ambiguity output for each $r$, as shown in Fig. 4-11. We see that the mainlobe shape is about the same, while the sidelobe levels are significantly increased. Some of the sidelobe positions are also shifted.

Fig. 4-12 gives another example for source range estimation with uncertainty of 


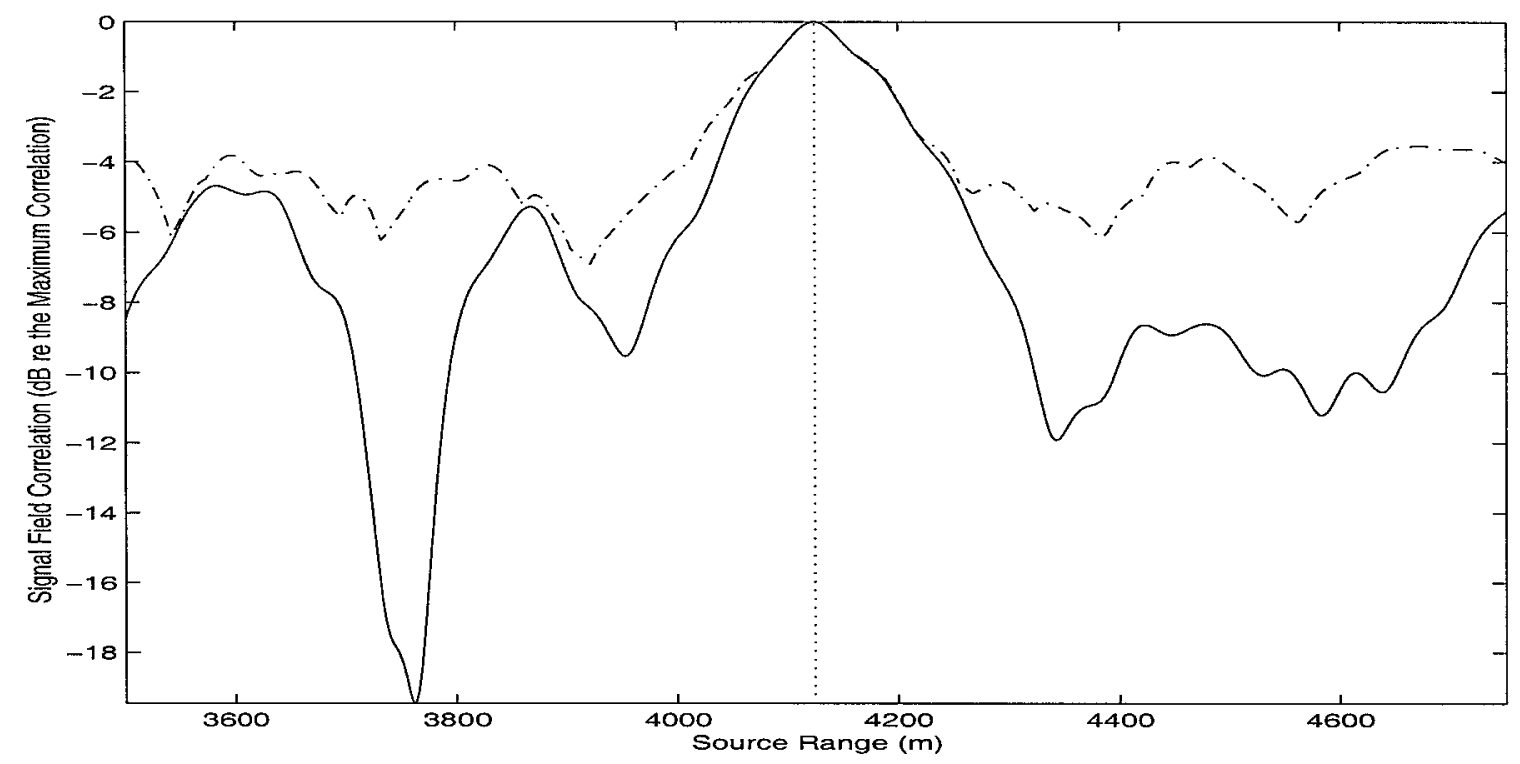

Figure 4-11: Signal ambiguity function for source range estimation without depth uncertainty (solid line) and with depth uncertainty (dashdot line). The dotted line indicates the true source range.

the sediment bottom wave-speed. The dotted line is a projection of Fig. 4-10(b) by choosing the maximum ambiguity output for each $r$. Now we see that both the mainlobe and sidelobe levels are increased. This can be explained by noting that the sediment bottom wave-speed is strongly coupled to the source range, while the source depth is not.

Therefore, in a multiple-parameter estimation problem, uncertainties of the other parameters could remarkably change the ambiguity structure in estimation of the chosen parameter, normally by increasing the mainlobe/sidelobe levels and/or shifting the sidelobe positions, depending on the parameter coupling.

\subsection{Maximum Likelihood Error Analysis Based on Two-Point Parameter Estimation}

We have seen that a large-error performance bound is closely related to the ambiguities, and the threshold phenomenon occurs when the sidelobe contribution to the total error is beyond the mainlobe contribution. To understand some fundamental 


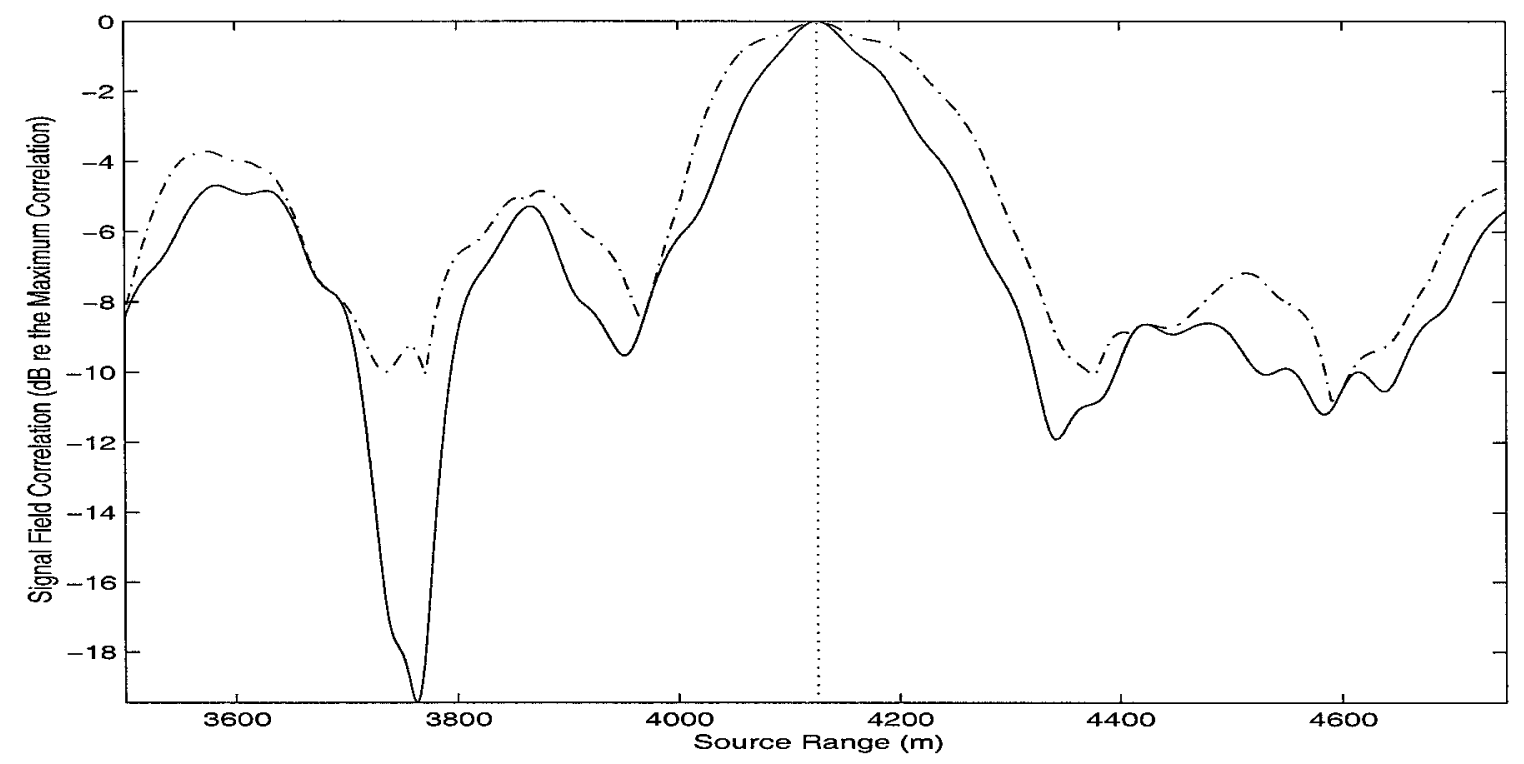

Figure 4-12: Signal ambiguity function for source range estimation without sediment bottom wave-speed uncertainty (solid line) and with sediment bottom wave-speed uncertainty (dashdot line). The dotted line indicates the true source range.

ambiguity behaviors and check the validity of the bounds, we would have estimation error at each scanning parameter point for a given true parameter point; errors at different mainlobe/sidelobe points can then be compared. In this section, we develop such an approach for error analysis in the context of the maximum likelihood estimate.

For the given matched-field data model, the MLE for source/environmental parameter estimation is given by (cf. Appendix B)

$$
\hat{\boldsymbol{\theta}}(\mathbf{R})=\arg \max _{\boldsymbol{\theta}} \sum_{m=1}^{M} \sum_{l=1}^{L}\left|\mathbf{R}_{l}^{\dagger}\left(f_{m}\right) \mathbf{g}\left(f_{m}, \boldsymbol{\theta}\right)\right|^{2} .
$$

Consider a discrete set of parameter values, $\left\{\boldsymbol{\theta}_{i}\right\}$. To analyze the performance of the maximum likelihood estimate, one may want to find the probability that the peak of the MLE output could occur at any particular candidate parameter point, that is, given $\boldsymbol{\theta}$ is the true parameter point,

$$
\operatorname{Pr}\left(\arg \max _{\boldsymbol{\theta}_{s}} \sum_{m=1}^{M} \sum_{l=1}^{L}\left|\mathbf{R}_{l}^{\dagger}\left(f_{m}\right) \mathbf{g}\left(f_{m}, \boldsymbol{\theta}_{s}\right)\right|^{2}=\boldsymbol{\theta}_{i}\right),
$$


where $\boldsymbol{\theta}_{s}$ denotes the scanning parameter point. Then the mean square error can be directly computed by

$$
\Sigma=\sum_{i} \sum_{j}\left(\hat{\boldsymbol{\theta}}_{j}-\boldsymbol{\theta}_{i}\right)\left(\hat{\boldsymbol{\theta}}_{j}-\boldsymbol{\theta}_{i}\right)^{T} p\left(\hat{\boldsymbol{\theta}}_{j} \mid \boldsymbol{\theta}_{i}\right) p\left(\boldsymbol{\theta}_{i}\right)
$$

This is for the random parameter model. If $\boldsymbol{\theta}$ is treated as a deterministic parameter, then the sum over $\boldsymbol{\theta}$ is removed. Unfortunately, it is very difficult, if not impossible, to solve the error probability in (4.26).

An estimation problem with a discrete set of parameter points can be transformed to an M-ary detection problem. The error analysis of an M-ary detection problem is still hard, and a union bound of performance [81] is often used based on a further simplified problem, the two-point estimation problem, as stated below.

Two-Point Parameter Estimation Problem: Given the data model in (3.28) and two possible parameter sets, $\boldsymbol{\theta}_{0}$ and $\boldsymbol{\theta}_{1}$, and given the observations in (3.30), we are to find estimates of the parameter set using the MLE in (4.25).

Under this definition, the error probability in parameter estimation involves the probability that given $\boldsymbol{\theta}_{0}\left(\boldsymbol{\theta}_{1}\right)$ is the true parameter, the MLE output at $\boldsymbol{\theta}_{1}\left(\boldsymbol{\theta}_{0}\right)$ is larger than that at $\boldsymbol{\theta}_{0}\left(\boldsymbol{\theta}_{1}\right)$, that is

$$
P_{e}\left(\boldsymbol{\theta}_{1} \mid \boldsymbol{\theta}_{0}\right)=\operatorname{Pr}\left(\sum_{m=1}^{M} \sum_{l=1}^{L}\left|\mathbf{R}_{l}^{\dagger}\left(f_{m}\right) \mathbf{g}\left(f_{m}, \boldsymbol{\theta}_{1}\right)\right|^{2}>\sum_{m=1}^{M} \sum_{l=1}^{L}\left|\mathbf{R}_{l}^{\dagger}\left(f_{m}\right) \mathbf{g}\left(f_{m}, \boldsymbol{\theta}_{0}\right)\right|^{2}\right)
$$

The two-point estimation error probability in (4.28) has been derived in Appendix $\mathrm{C}$ and summarized in Section 3.2.4. Fig. 4-13 shows examples of $P_{e}\left(\boldsymbol{\theta}_{1} \mid \boldsymbol{\theta}_{0}\right)$ for source range estimation in Section 4.1.1. As discussed in Section 4.3.3, size of the error probability across the parameter interval exactly follows the signal field correlation level (cf. Fig. 4-2(d)). This is more visible at low SNR levels because the relative difference in error probability associated with a high-correlation-level point and a low-correlation-level point is decreased. We also notice that the maximum error probability is 0.5 . This must be the case because, for a perfectly known environment, 

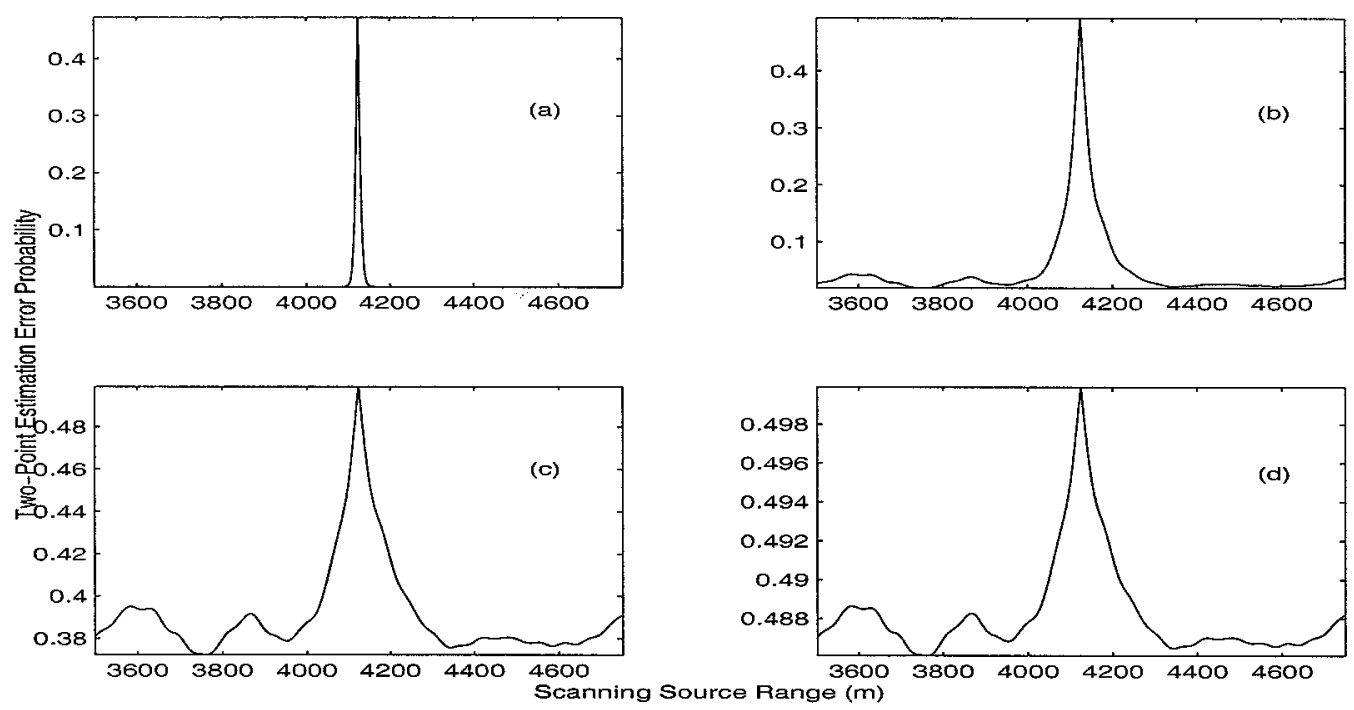

Figure 4-13: Two-point estimation error probability associated with each scanning source range and the true source range: (a) $\mathrm{SNR}=0 \mathrm{~dB}$; (b) SNR $=-10 \mathrm{~dB}$; (c) SNR $=-20 \mathrm{~dB} ;(\mathrm{d}) \mathrm{SNR}=-30 \mathrm{~dB}$. The true source range is at $4125 \mathrm{~m}$.

the signal field correlation achieves the maximum at the true parameter position.

\subsubsection{Estimation Errors due to Different Ambiguity Points}

Consider a set of scanning parameter points, $\left\{\boldsymbol{\theta}_{1}, \boldsymbol{\theta}_{2}, \ldots\right\}$, and suppose the true parameter point is one of them, $\boldsymbol{\theta}_{j}$. Denote $\varepsilon_{j}$ as the event that $\hat{\boldsymbol{\theta}} \neq \boldsymbol{\theta}_{j}$ and $\varepsilon_{k j}$ as the event that the MLE output at $\boldsymbol{\theta}_{k}$ is larger than that at $\boldsymbol{\theta}_{j}$ for $k \neq j$. From (4.25), we see that $\varepsilon_{j}$ is a union, for $k \neq j$, of $\varepsilon_{k j}$. Therefore, the error probability is given by [81, page 104]:

$$
\begin{aligned}
\operatorname{Pr}\left(\varepsilon_{j} \mid \boldsymbol{\theta}_{j}\right)= & \operatorname{Pr}\left(\bigcup_{k \neq j} \varepsilon_{k j} \mid \boldsymbol{\theta}_{j}\right) \\
= & \sum_{k \neq j} \operatorname{Pr}\left(\varepsilon_{k j} \mid \boldsymbol{\theta}_{j}\right) \\
& -\sum_{k \neq j, i \neq j, k \neq i} \operatorname{Pr}\left(\varepsilon_{k j} \bigcap \varepsilon_{i j} \mid \boldsymbol{\theta}_{j}\right) \\
& +\ldots
\end{aligned}
$$


The first term of the second equality is the so-called union bound, which is an upper bound to the desired probability.

Obviously, each $\varepsilon_{k j}$ corresponds to a two-point estimation problem. Because these two-point estimation events are not mutually exclusive, we cannot get an exact error probability for each scanning parameter point. However, given the true parameter point at $\boldsymbol{\theta}_{j}$, the exact error probability at any scanning parameter point $\boldsymbol{\theta}_{k}$ is proportional to the two-point estimation error probability associated with $\boldsymbol{\theta}_{j}$ and $\boldsymbol{\theta}_{k}$, by the first-order approximation of (4.29). This is a good approximation in the intermediate to high SNR region, where the second-order terms in (4.29) can often be ignored.

A quantitative error analysis is then available. We first compute $P_{e}\left(\boldsymbol{\theta}_{k} \mid \boldsymbol{\theta}_{j}\right)$ for each $\boldsymbol{\theta}_{k}, k \neq j$, at different SNR levels, and then multiply them by the corresponding distance square, $\left(\boldsymbol{\theta}_{k}-\boldsymbol{\theta}_{j}\right)^{2}$. So we have the estimation error at each scanning parameter point, that is

$$
\epsilon_{M L E}^{2}\left(\boldsymbol{\theta}_{k} \mid \boldsymbol{\theta}_{j}\right)=P_{e}\left(\boldsymbol{\theta}_{k} \mid \boldsymbol{\theta}_{j}\right) \times\left(\boldsymbol{\theta}_{k}-\boldsymbol{\theta}_{j}\right)^{2}
$$

We define this point estimation error as the probabilistic square error.

Consider again the example of source range estimation. Suppose that the true source range is $4125 \mathrm{~m}$ and the scanning interval is $3500 \mathrm{~m}$ to $4750 \mathrm{~m}$. This interval is discretized by

$$
\boldsymbol{\theta}_{i}=3500+(i-1) \times 1 \mathrm{~m}, \quad i=1, \ldots, 1251
$$

Obviously, the true parameter is $\boldsymbol{\theta}_{626}$. The probabilistic square errors at different scanning parameter points are evaluated, as shown in Fig. 4-14, for which ten independent snapshots are used.

Fig. 4-14 demonstrates several operation regions with respect to SNR. For SNR above $7 \mathrm{~dB}$, the errors around the mainlobe peak dominate. Below SNR $=7 \mathrm{~dB}$, distant mainlobe points introduce larger errors, and the errors at some sidelobe points become visible. The sidelobe errors are comparable to the mainlobe errors at SNR $=-2 \mathrm{~dB}$, and even beyond the mainlobe errors as the SNR further decreases. Below 


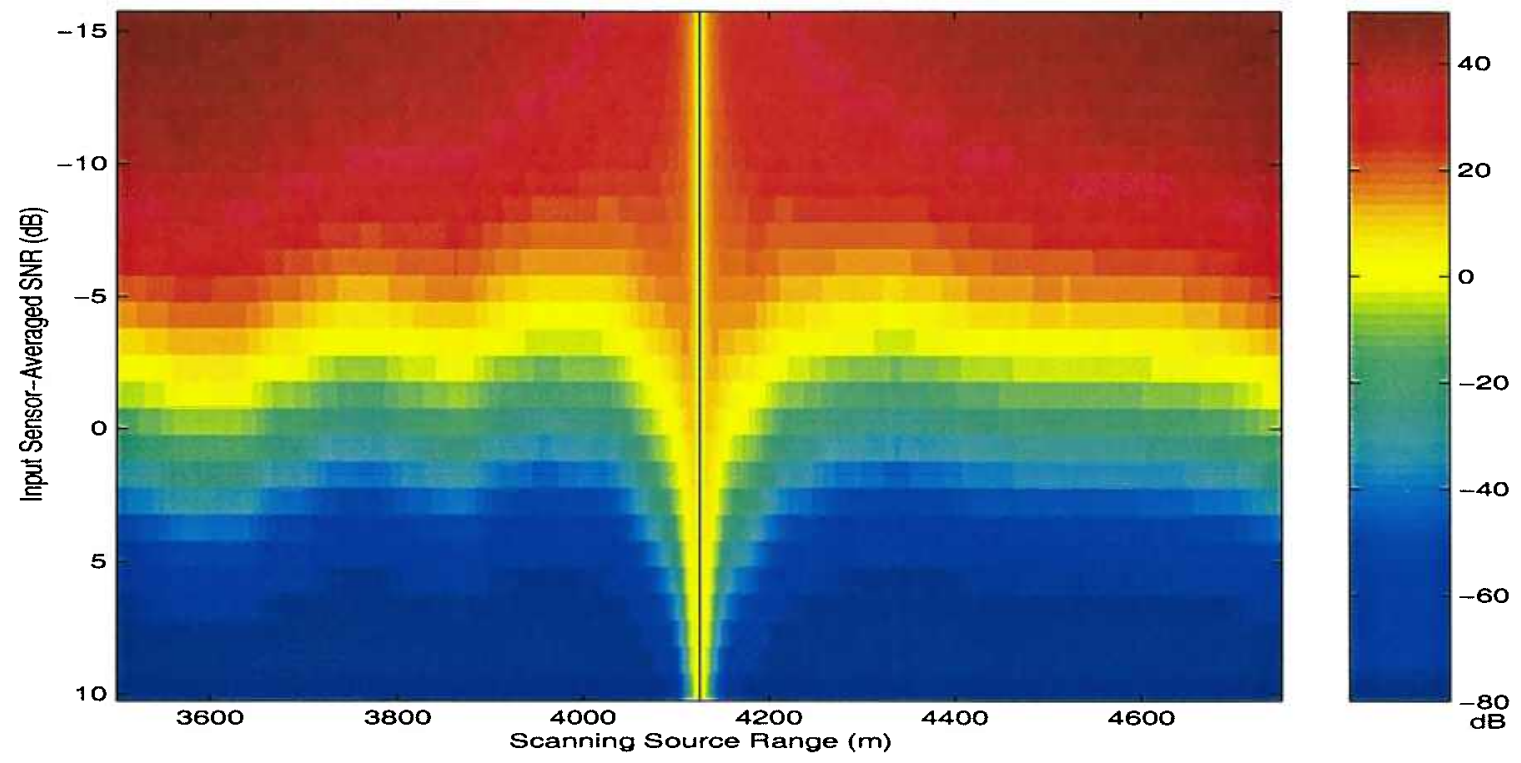

Figure 4-14: Probabilistic square error as a function of SNR at each scanning source range. The vertical line in the middle (with zero error) corresponds to the true source range.

SNR $=-8 \mathrm{~dB}$, the differences in error probability associated with different ambiguity points are so small that the probabilistic square errors are gradually dominated by the distance between the scanning parameter point and the true parameter point. In summary, this plot describes some fundamental behaviors in this parameter estimation problem.

To look at some detailed behaviors, we first plot the probabilistic square errors at different sidelobe points as shown in Fig. 4-15. As expected, the highest sidelobe peak at $3583 \mathrm{~m}$ (cf. Fig 4-2(d)) leads to larger errors, about $10 \mathrm{~dB}$ higher than those at $3867 \mathrm{~m}$ and $4479 \mathrm{~m}$. That the result at $4750 \mathrm{~m}$ is close to (and even a bit beyond at very low SNR) that at $3583 \mathrm{~m}$ is due to a larger distance. Even so, the curve for the sidelobe peak at $3583 \mathrm{~m}$ represents the dominating sidelobe behavior well. This behavior is not very sensitive to the accuracy of the sidelobe peak location. As shown in Fig. 4-16, errors at the sidelobe peak $(3583 \mathrm{~m})$ are very close to those at $\pm 50 \mathrm{~m}$ from the peak particularly in the low to intermediate SNR region. This is the region of interest regarding the sidelobe issue.

For the mainlobe behavior, we first notice that the close neighboring point(s) of the 


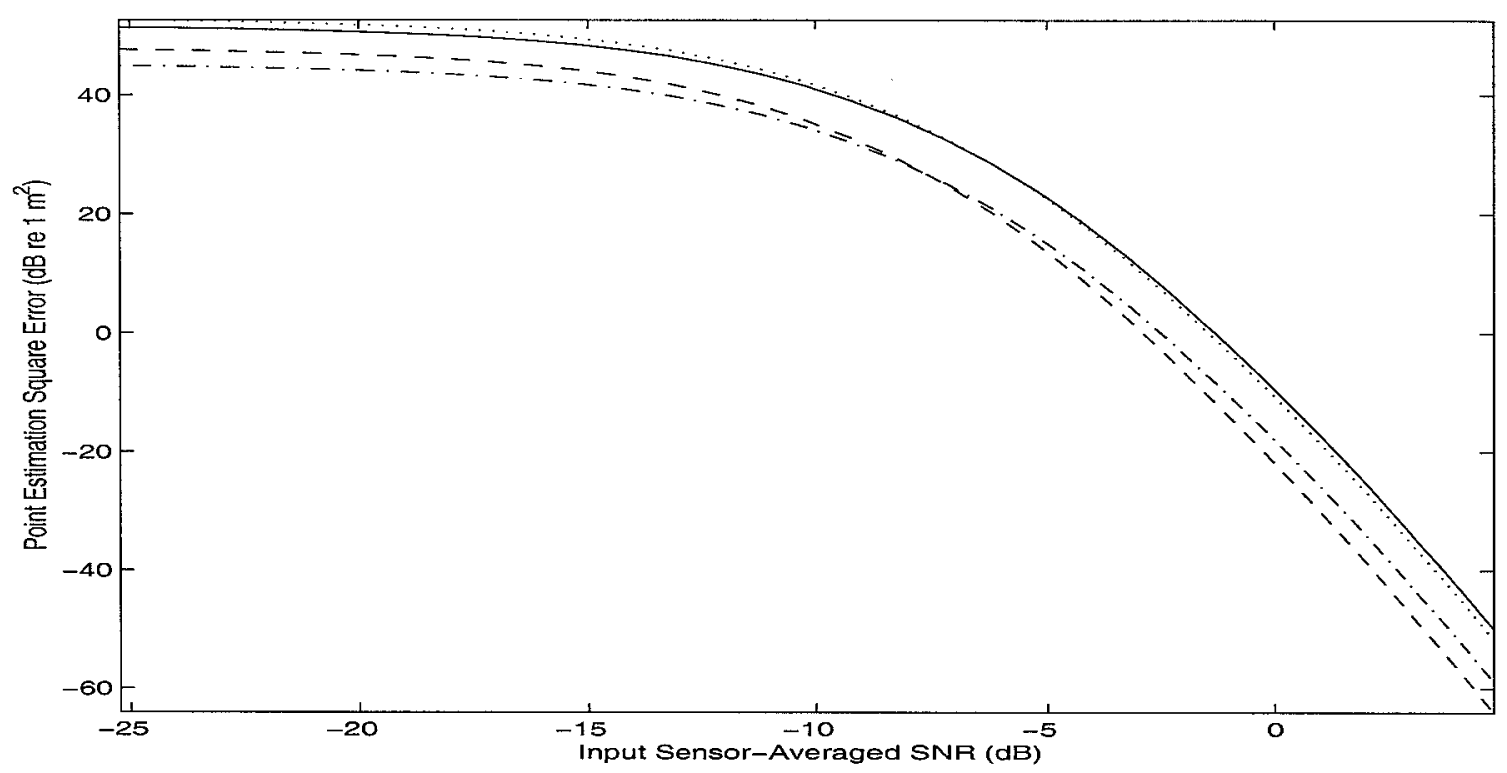

Figure 4-15: Probabilistic square errors as a function of SNR at different sidelobe peak points: $3583 \mathrm{~m}$ (solid line); $3867 \mathrm{~m}$ (dashdot line); $4479 \mathrm{~m}$ (dashed line); and $4750 \mathrm{~m}$ (dotted line).

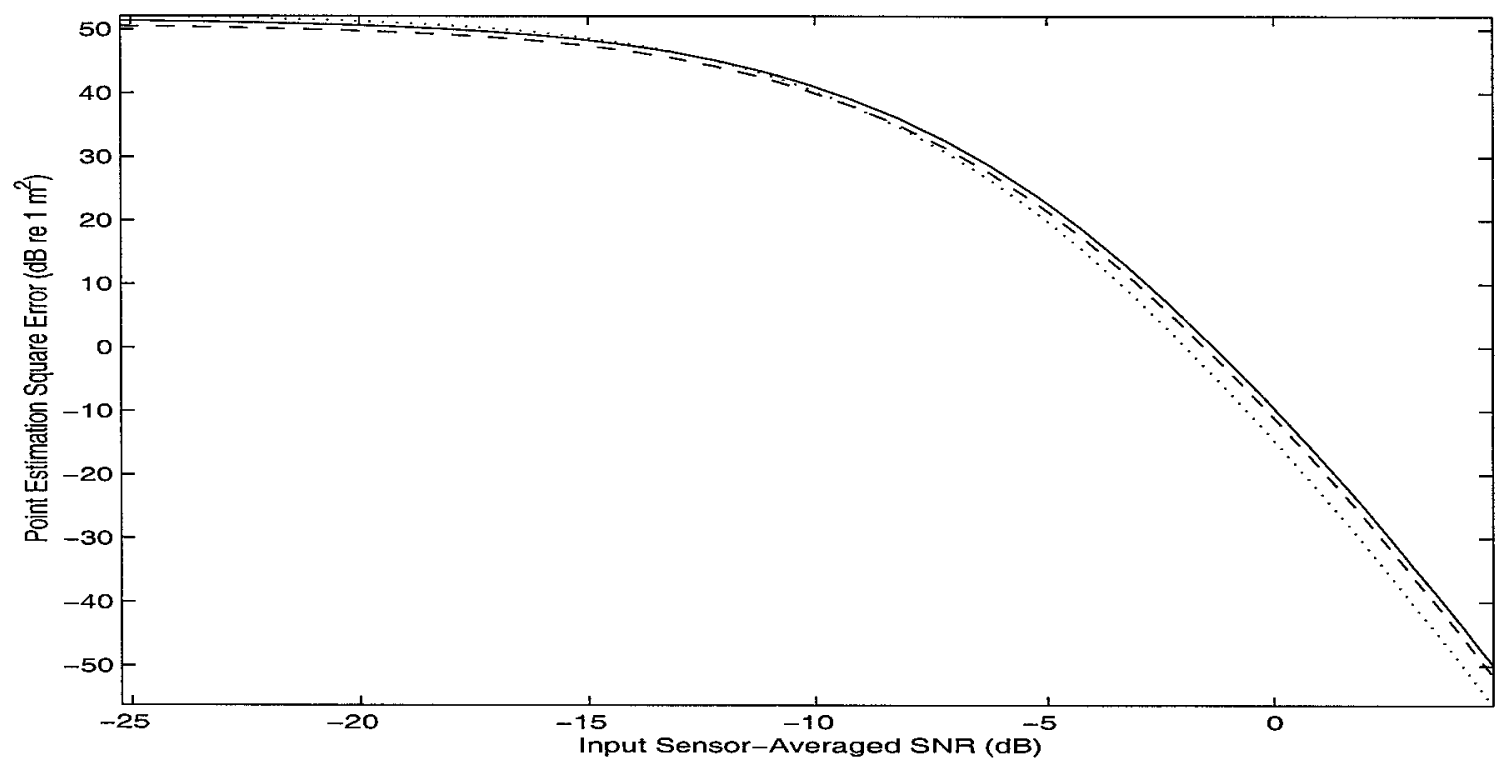

Figure 4-16: Probabilistic square errors as a function of SNR at different sidelobe points around a sidelobe peak: $3583 \mathrm{~m}$ (solid line); $3533 \mathrm{~m}$ (dotted line); and $3633 \mathrm{~m}$ (dashed line). 


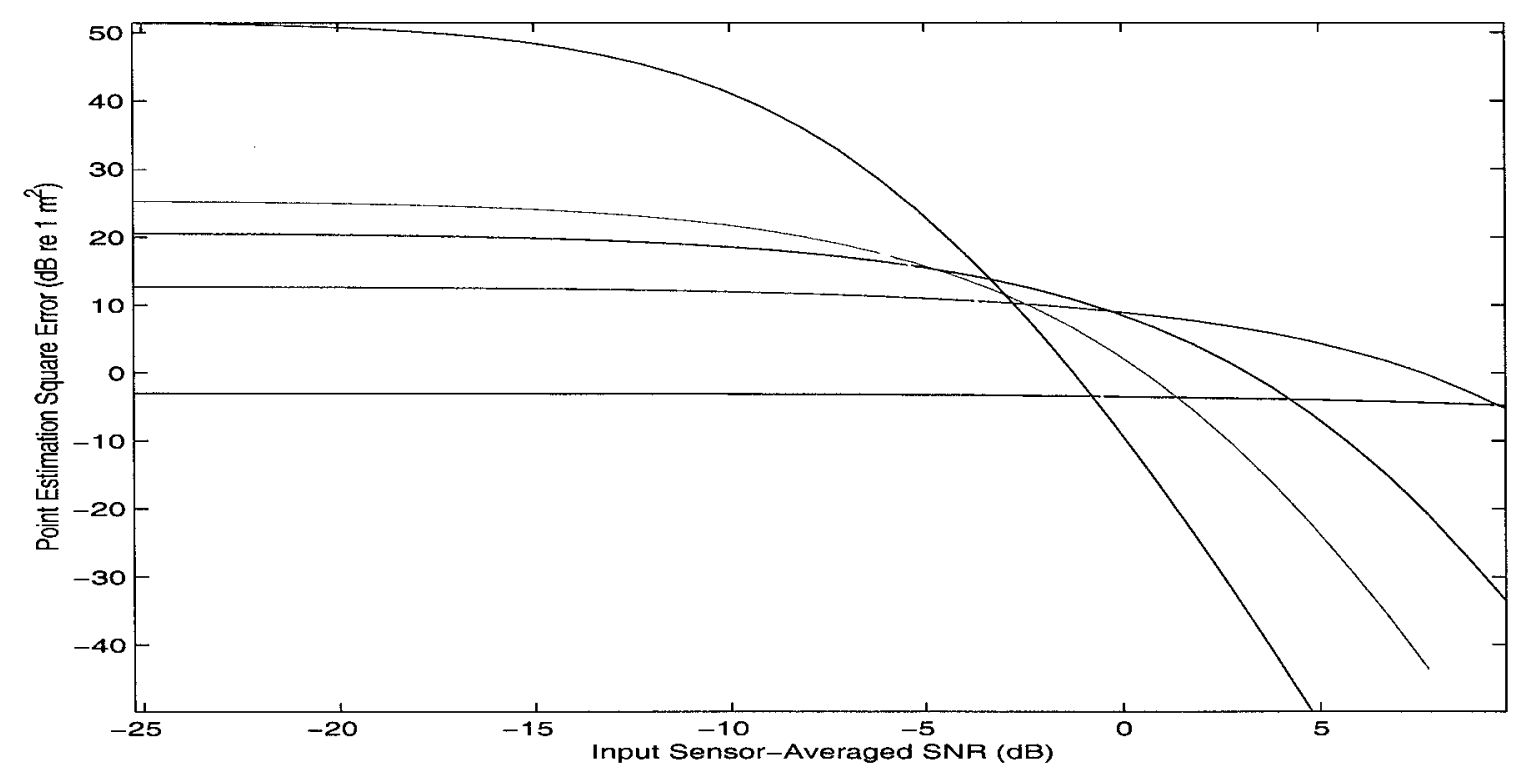

Figure 4-17: Probabilistic square errors as a function of SNR at different mainlobe points: $4124 \mathrm{~m}$ (black line); $4119 \mathrm{~m}$ (magenta line); $4110 \mathrm{~m}$ (blue line); $4099 \mathrm{~m}$ (green line); and $4079 \mathrm{~m}$ (yellow line). The result for the sidelobe peak at $3583 \mathrm{~m}$ is also shown (red line).

true parameter usually plays a key role in the very high SNR region. This is plotted in Fig 4-17 together with other mainlobe points. We see an obvious counter-interaction between the field correlation level and the parameter distance. The probabilistic square error at a close mainlobe point is larger at high SNR, but increases slowly as the SNR decreases and is soon below the error at a distant mainlobe point, which is smaller at high SNR but increases rapidly. For comparison, the result at the representative sidelobe point $(3583 \mathrm{~m})$ is also included. At $\mathrm{SNR} \approx-3 \mathrm{~dB}$, the sidelobe error is over all the mainlobe errors. The intersection point is specified by this sidelobe point and the mainlobe point at $4110 \mathrm{~m}$. We denote this mainlobe point as the representative distant mainlobe point. Apparently, the representative mainlobe point depends on not only the mainlobe shape (correlation level) but also the relative magnitude of the mainlobe and sidelobe levels.

So the representative ambiguity point can be identified at each SNR by locating the peak probabilistic square error. Fig. 4-18 summarizes the results in Figs. 4-15 and $4-17$ by plotting the errors at three representative ambiguity points for different operation regions. Typically, above $\mathrm{SNR}_{1}$, the errors of the mainlobe points dominate; 


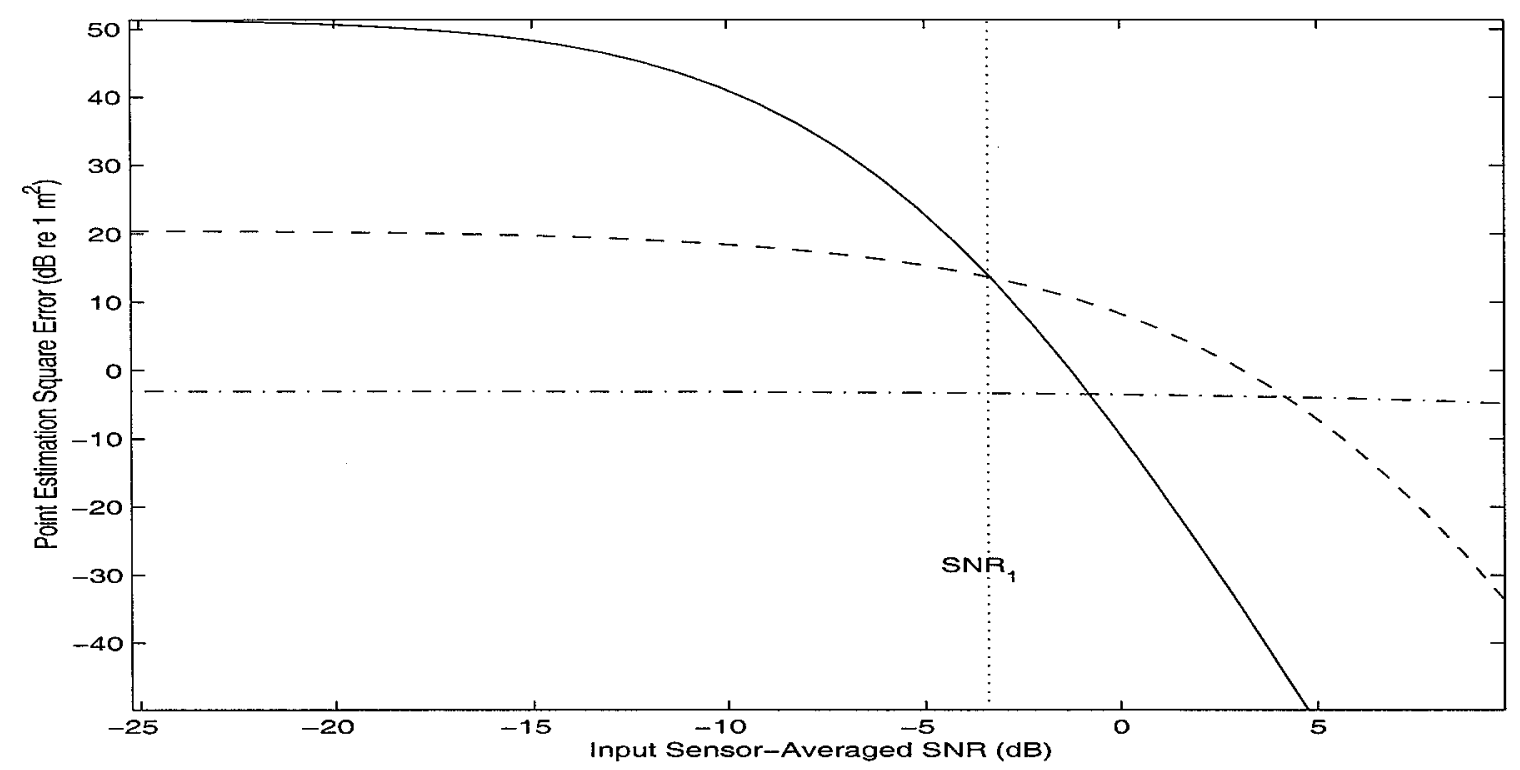

Figure 4-18: Probabilistic square errors as a function of SNR at different ambiguity points: close mainlobe point (4124 m, dashdot line); distant mainlobe point (4110 m, dashed line); and distant sidelobe point (3583 $\mathrm{m}$, solid line).

below $\mathrm{SNR}_{1}$, the sidelobe contribution is the major source of the estimation error. As discussed in Section 4.3.2 and 4.3.3, the larger-error performance bounds demonstrate exactly the same behaviors regarding the error contributions from different ambiguity points.

\subsubsection{Local Performance Approximation}

To check the validity of the developed error analysis, the two-point estimation error probability is used to obtain some approximations to the mean square error of the maximum likelihood estimate for a fixed true parameter. This corresponds to a deterministic (but unknown) parameter model, and the performance measure is the local mean square error. Note that in the high SNR region, the peak of the true parameter protrudes prominently above the noise and can be located accurately. The estimation error is due to slight, noise-induced distortion of the true peak and can be well predicted by the CRB. In the low SNR region, the true peak could be below the noise level and obscured by other ambiguous peaks. The performance is dominated by choosing the interval the true peak lies in, and a large error arises when a wrong 
interval is selected.

Under this understanding, a natural way to approximate the mean square error is [72]:

$$
\begin{aligned}
\varepsilon^{2}(\theta)= & E\left[(\hat{\theta}-\theta)^{2}\right] \\
= & E\left[(\hat{\theta}-\theta)^{2} \mid \text { interval error }\right] \operatorname{Pr}(\text { interval error }) \\
& +E\left[(\hat{\theta}-\theta)^{2} \mid \text { no interval error }\right] \operatorname{Pr}(\text { no interval error }) .
\end{aligned}
$$

In Ref. [72], although the parameter interval was divided into multiple (say, $M$ ) subintervals to form an M-ary detection problem, different sidelobe errors corresponding to different sub-intervals were uniformly treated. As we see in Fig. 4-15, the errors due to different sidelobe points could differ up to $10 \mathrm{~dB}$. To achieve a better approximation, we would resort to a true M-ary detection scheme.

We first divide the parameter interval into multiple sub-intervals so that, except the mainlobe sub-interval, each sub-interval contains an apparent sidelobe structure. For example, for the source range estimation problem in Fig. 4-2, there is one mainlobe sub-interval and four sidelobe sub-intervals as specified in Fig. 4-19. We then denote each sub-interval by the sidelobe peak point, $\theta_{i}, i=1, \ldots, M-1$, and use the two-point estimation error probability, $P_{e}\left(\theta_{i} \mid \theta\right)$ as the probability that an estimate falls into this sub-interval. We also notice that $E\left[(\hat{\theta}-\theta)^{2} \mid\right.$ no interval error $]$ is just the local CRB. Then the mean square error can be approximated by

$$
\begin{aligned}
\varepsilon^{2}(\theta)= & \left(1-\sum_{i=1}^{M-1} P_{e}\left(\theta_{i} \mid \theta\right)\right) \times \mathrm{CRB} \\
& +\sum_{i=1}^{M-1} P_{e}\left(\theta_{i} \mid \theta\right) \times\left(\theta_{i}-\theta\right)^{2} .
\end{aligned}
$$

Fig. 4-20 presents the results for the example in Fig. 4-19. Also plotted are the local CRB in (2.8) and the MLE simulation results using (4.25). The approximation agrees very well with the MLE simulation in the intermediate to high SNR region. For very low SNR, the approximation is bit high because the two-point error probability. 


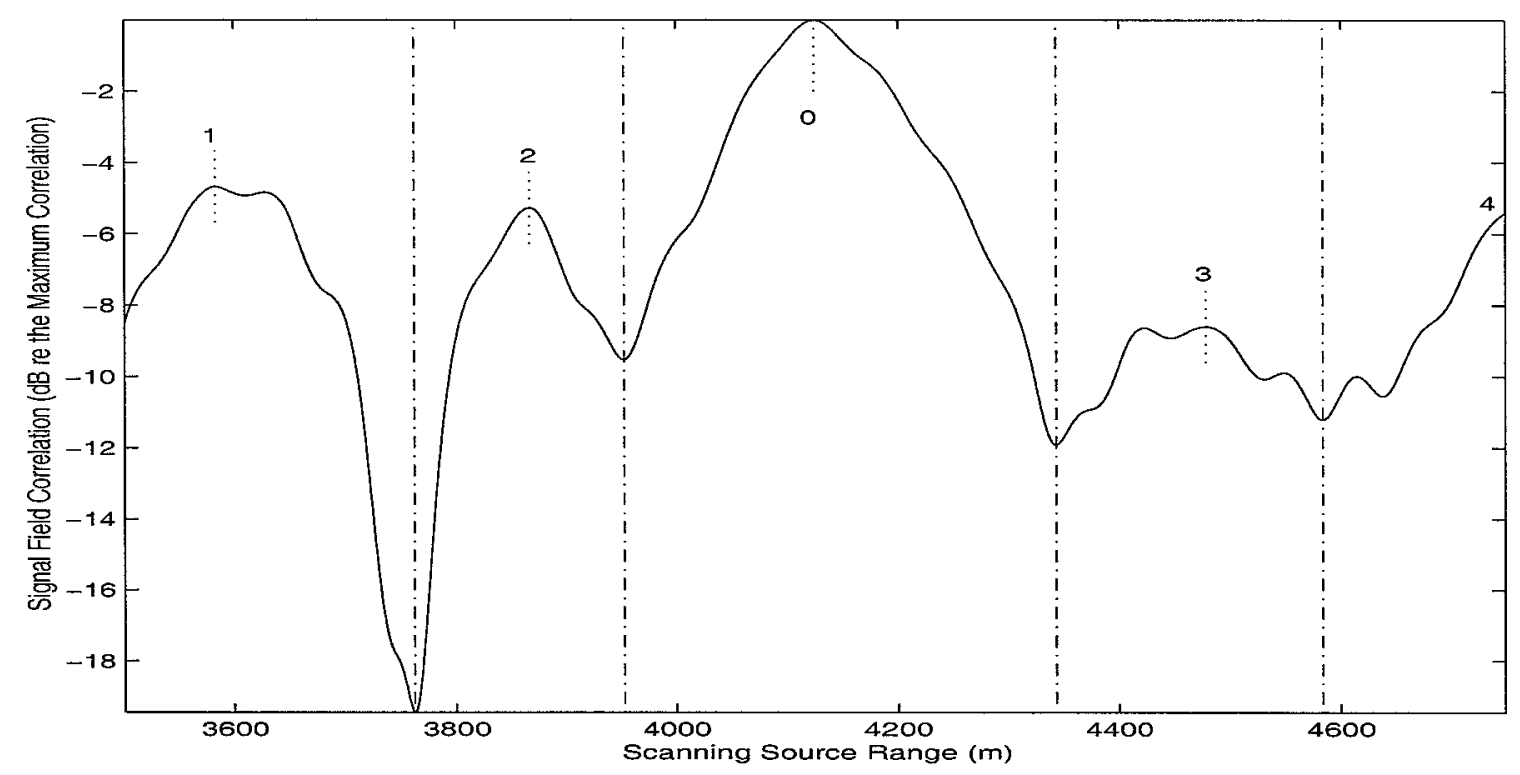

Figure 4-19: Division of the parameter interval for definition of the M-ary detection problem based on signal ambiguity function in source range estimation. The true source range is at $4125 \mathrm{~m}$. The dashdot line indicates the separation between two adjacent sub-intervals. The dotted line indicates the mainlobe/sidelobe peak position.

over-states the exact MLE error probability. In contrast, the local CRB is good only at high SNR.

To summarize this section, we establish a connection between the errors in parameter estimation and the field ambiguities through the two-point estimation error probability. The proposed approach makes it possible to quantitatively analyze the ambiguities in the matched-field problem and obtain a close approximation to the estimation error. Discussions in this section are for single parameter estimation. For vector parameter estimation, the mainlobe/sidelobe properties (for example, size, shape and position) are addressed in a multi-dimensional space. However, generalization of the above analysis to multiple parameter estimation is straightforward, although complicated (cf. Sections 6.4 and 6.5 for some examples). The proposed approach can also be applied to random parameter estimation by averaging over individual parameter points. The next section gives such an example for determining the threshold SNR. 


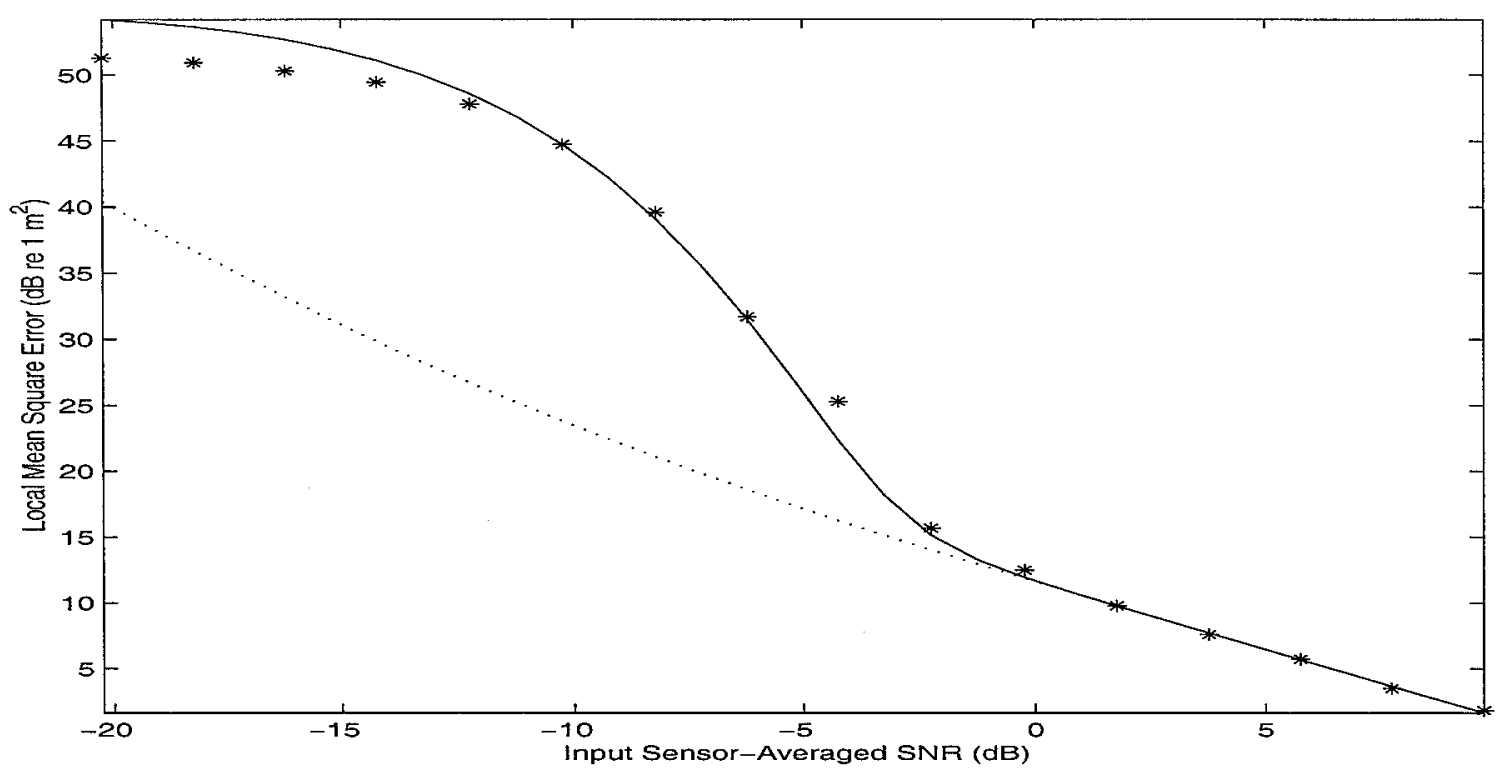

Figure 4-20: Mean square error as a function of SNR for source range estimation given by the local CRB (dotted line), the local approximation in (4.33) (solid line), and the MLE simulations $\left(^{*}\right)$. The true source range is at $4125 \mathrm{~m}$.

\subsection{Threshold Signal-to-Noise Ratio}

In the last section, we have seen that the ambiguity behavior shows a strong SNRdependence. As a result, the performance often demonstrates several distinct regions of operation in terms of the SNR, as shown in Fig. 4-20. To describe such a performance, we need to specify the threshold SNR's between different operation regions. This can be done by evaluating a large-error performance bound, because, as discussed in Section 4.3, such bounds capture the parameter estimation ambiguities at different SNR levels, leading to tighter performance predictions in all SNR regions.

The canonical behavior of a large-error performance bound is described in Fig. 421. There are three distinct regions of operation: (1) the asymptotic region at high SNR determined by the mainlobe ambiguity; (2) the no-information region at low SNR due to the noise domination; and (3) the transition region in the middle attributed to the sidelobe ambiguity.

In the asymptotic region, the mean square error $\left(\epsilon^{2}\right.$, expressed in $\left.\mathrm{dB}\right)$ is linear with respect to the SNR (also expressed in $\mathrm{dB}$ ). The threshold phenomenon can be described as a departure from the linear SNR-dependence. Thus, one may define the 


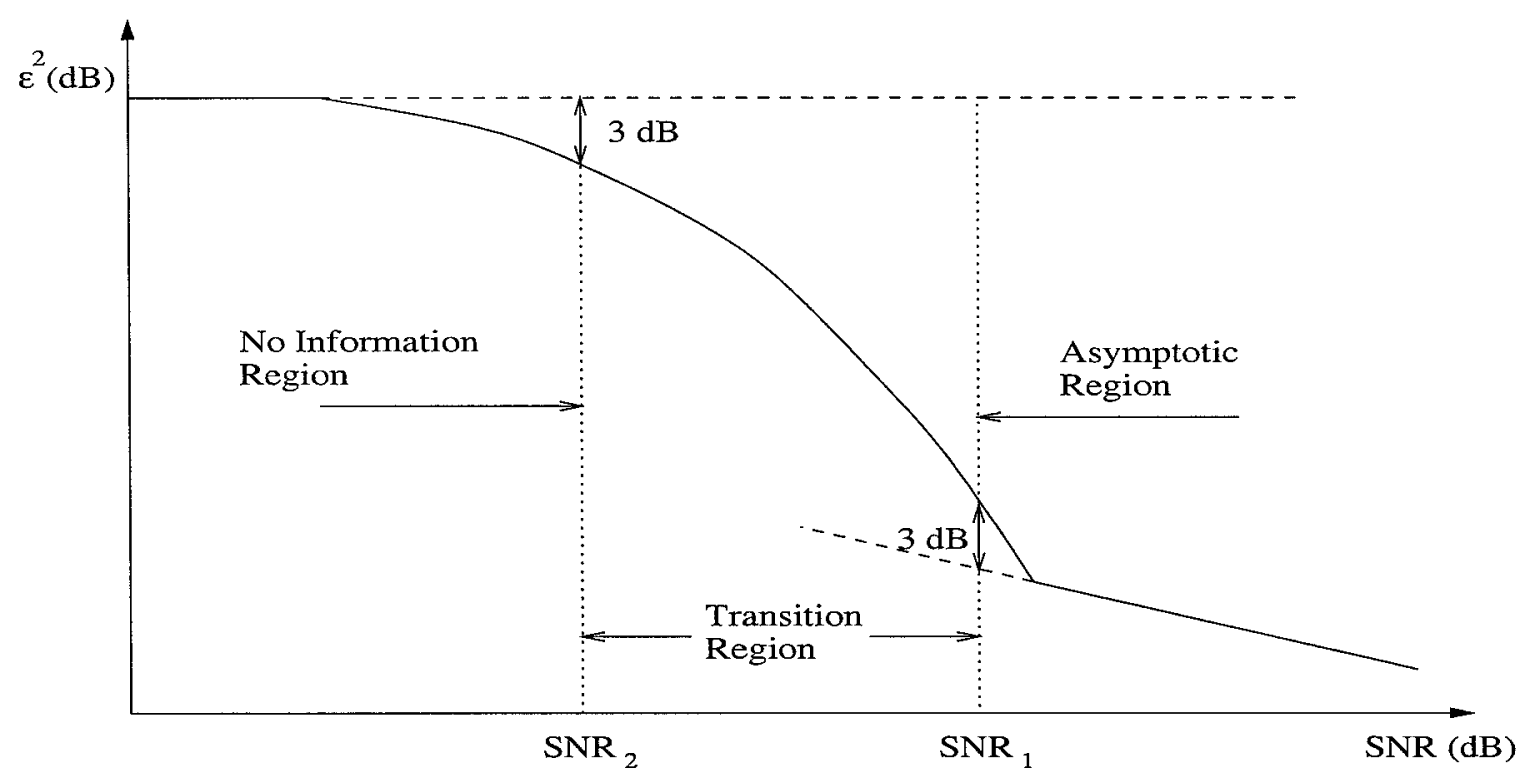

Figure 4-21: Typical larger error performance bound: mean square error versus SNR.

threshold SNR between the asymptotic region and the transition region, $\mathrm{SNR}_{1}$, as the point at which the bound is $3 \mathrm{~dB}$ higher than the linear prediction. Let us denote the linear-dependence of the asymptotic bound as $b-a \cdot \mathrm{SNR}$, where $b$ is the error prediction at SNR $=0 \mathrm{~dB}$, and $a$ is the slope of this linear-dependence. Obviously, both $a$ and $b$ are positive. Then we have for $\mathrm{SNR}_{1}$

$$
\epsilon^{2}\left(\mathrm{SNR}_{1}\right)=b-a \cdot \mathrm{SNR}_{1}+3 .
$$

Because of the complicated parameter-dependence of the signal field, we don't have a simple closed-form expression for either WWB or ZZB. (4.34) has to be solved numerically. One may first evaluate the bound at several (three or more) high SNR points, based on which values of $a$ and $b$ are estimated using linear fitting. Then the bound is evaluated at gradually decreased SNR levels until (4.34) is satisfied.

The threshold SNR between the transition region and the no-information region, $\mathrm{SNR}_{2}$, can be similarly defined. Note that in the no-information region, the mean square error is almost a constant close to the variance of the random parameter distribution. So $\mathrm{SNR}_{2}$ can be defined as the point at which the bound is $3 \mathrm{~dB}$ lower than the a priori parameter variance. For a uniform parameter distribution over 
$[-\tau, \tau]$, we have

$$
\epsilon^{2}\left(\mathrm{SNR}_{2}\right)=10 \cdot \log _{10}\left(\frac{\tau^{2}}{3}\right)-3
$$

which is again solved in a numerical way.

The optimum test points of the WWB can be used to aid the search for $\mathrm{SNR}_{1}$ and $\mathrm{SNR}_{2}$. As mentioned in Section 4.3.2, the optimum test point switches from a mainlobe point to a sidelobe point around the threshold SNR. Hence, we may pre-select a set of representative mainlobe points and sidelobe points and evaluate the WWB at these points only. When the bound at any sidelobe point is beyond those at all mainlobe points at some SNR level, we may use that SNR point as the approximation to $\mathrm{SNR}_{1}$ and then a further refinement can be easily followed. When the bound at one-third of the parameter interval (cf. Section 4.3.2) is the highest at a low SNR level, we may use that $\mathrm{SNR}$ as an approximation to $\mathrm{SNR}_{2}$.

In evaluation of the ZZB, the behavior of the integrand in (3.23) as a function of the parameter perturbation is similar to the WWB as a function of the test point. So we may evaluate the integrand for a pre-selected set of parameter perturbations

representing main mainlobe and sidelobe points. Approximations to $\mathrm{SNR}_{1}$ and $\mathrm{SNR}_{2}$ are obtained in a similar way.

The above approach requires evaluation of the performance bound at all or part of test points (perturbations). For $\mathrm{SNR}_{1}$, an alternative approach is available by exploiting the ambiguity analysis based on the two-point estimation error probability. This approach only requires some coarse information on the signal field correlation and has a simple physical interpretation and simple implementation.

\subsubsection{Estimation of Threshold SNR Based on the Two-Point Estimation Error Analysis}

Recall that in Section 4.4, although not explicitly stated, we have already presented an approach to find the threshold SNR for a local parameter estimation problem. Given the signal field ambiguity function, we first locate the most important sidelobe point 
as well as the representative mainlobe point. Then we plot the probabilistic square errors as a function of the SNR. The sidelobe error is over the mainlobe error at the intersection point, and thus, per our understanding on the threshold phenomenon, this point actually corresponds to the threshold SNR. Indeed, in the example of source range estimation (cf. Fig. 4-18), the intersection between the mainlobe error curve and the sidelobe error curve occurs at $\mathrm{SNR}=-3 \mathrm{~dB}$. This is a quite good indication of the threshold SNR observed from the MLE simulations (Fig. 4-20).

For the random parameter model assumed by Bayesian performance bounds, one may want to repeat those steps for each parameter point within the parameter interval (usually represented by a discrete set). The ambiguity functions within a small sub-interval often demonstrate similar structures regarding the mainlobe shape, and sidelobe levels and positions. Moreover, as observed in Section 4.4, the probabilistic square error is not sensitive to some fine sidelobe structures. As a result, the predicted SNR often shows a small variation across the parameter interval.

Under this consideration, we only need to solve the threshold SNR for a limited set of parameter points. The threshold SNR for the entire parameter space can then be taken as the average of these individual threshold SNR's. However, when the performance is averaged over a parameter interval, it is often strongly influenced by large errors at some parameter points. Therefore, a better threshold prediction may be taken as the $\alpha$-percentile point with $\alpha>50$.

Following the same example of source range estimation, we solve the threshold SNR for 24 uniformly-spaced parameter points covering the entire parameter interval. As given in Table 4.1, the threshold SNR ranges from $-2 \mathrm{~dB}$ to $-4 \mathrm{~dB}$ and the difference between any two adjacent threshold predictions is up to $1 \mathrm{~dB}$. We see the sidelobe ambiguity is quite uniform across the parameter space, only a bit less at long-range. This might be because some lower-order modes are more important at long-range. The mean of 24 threshold predictions is about $-3 \mathrm{~dB}$. If we choose the 83 -percentile point, the threshold SNR is $-2 \mathrm{~dB}$.

It is interesting to note that using a full column spanning array does decrease the threshold SNR. Table 4.2 gives individual threshold predictions for the 26 -sensor 
Table 4.1: Threshold SNR predictions at individual true parameter points in source range estimation.

\begin{tabular}{|c|r||c|r|}
\hline Source Range $(\mathrm{m})$ & $\mathrm{SNR}_{1}(\mathrm{~dB})$ & Source Range $(\mathrm{m})$ & $\mathrm{SNR}_{1}(\mathrm{~dB})$ \\
\hline \hline 3551 & -4 & 4151 & -3 \\
\hline 3601 & -3 & 4201 & -3 \\
\hline 3651 & -3 & 4251 & -3 \\
\hline 3701 & -2 & 4301 & -3 \\
\hline 3751 & -3 & 4351 & -3 \\
\hline 3801 & -3 & 4401 & -3 \\
\hline 3851 & -2 & 4451 & -4 \\
\hline 3901 & -2 & 4501 & -4 \\
\hline 3951 & -2 & 4551 & -3 \\
\hline 4001 & -3 & 4601 & -4 \\
\hline 4051 & -4 & 4651 & -4 \\
\hline 4101 & -3 & 4701 & -4 \\
\hline Mean Point & -3 & 83 -percentile Point & -2 \\
\hline
\end{tabular}

array (cf. Fig. 4-3(a)). The mean point is about $3 \mathrm{~dB}$ lower than the previous one for a short array; removing the added $2 \mathrm{~dB}$ array gain, the threshold SNR decreases by one dB. Similarly, the threshold SNR for the 16-sensor sparse array (corresponding to Fig. 4-3(b)) is also one dB lower (Table 4.3). Nonetheless, this improvement might be insignificant considering the increased deployment difficulty as well as cost.

\subsection{Summary}

The ambiguity analysis in this chapter mainly includes two aspects. First, we study the signal field correlation through the variations of individual source/environmental parameters. This helps to explain which factors cause the ambiguity in the matchedfield problem. Second, the effects of the ambiguity function are investigated first in relation to the behaviors of the performance bound, then in the context of the two-point MLE estimation error analysis. Particularly, introduction of the two-point estimation error probability establishes a direct connection between the errors in parameter estimation and the field ambiguities. These developments are well summarized through the application of locating the threshold SNR. 
Table 4.2: Threshold SNR predictions at individual true parameter points in source range estimation. A 26-sensor column spanning array is used.

\begin{tabular}{|c|r||c|r|}
\hline Source Range $(\mathrm{m})$ & $\mathrm{SNR}_{1}(\mathrm{~dB})$ & Source Range $(\mathrm{m})$ & $\mathrm{SNR}_{1}(\mathrm{~dB})$ \\
\hline \hline 3551 & -5 & 4151 & -6 \\
\hline 3601 & -5 & 4201 & -6 \\
\hline 3651 & -5 & 4251 & -6 \\
\hline 3701 & -5 & 4301 & -6 \\
\hline 3751 & -5 & 4351 & -6 \\
\hline 3801 & -6 & 4401 & -6 \\
\hline 3851 & -6 & 4451 & -6 \\
\hline 3901 & -6 & 4501 & -6 \\
\hline 3951 & -6 & 4551 & -6 \\
\hline 4001 & -6 & 4601 & -6 \\
\hline 4051 & -6 & 4651 & -6 \\
\hline 4101 & -6 & 4701 & -6 \\
\hline \hline Mean Point & -6 & & \\
\hline
\end{tabular}

Table 4.3: Threshold SNR predictions at individual true parameter points in source range estimation. A 16-sensor column spanning array is used.

\begin{tabular}{|c|r||c|r|}
\hline Source Range $(\mathrm{m})$ & $\mathrm{SNR}_{1}(\mathrm{~dB})$ & Source Range $(\mathrm{m})$ & $\mathrm{SNR}_{1}(\mathrm{~dB})$ \\
\hline \hline 3551 & -3 & 4151 & -4 \\
\hline 3601 & -4 & 4201 & -5 \\
\hline 3651 & -3 & 4251 & -4 \\
\hline 3701 & -4 & 4301 & -4 \\
\hline 3751 & -3 & 4351 & -4 \\
\hline 3801 & -3 & 4401 & -4 \\
\hline 3851 & -4 & 4451 & -4 \\
\hline 3901 & -4 & 4501 & -4 \\
\hline 3951 & -4 & 4551 & -4 \\
\hline 4001 & -4 & 4601 & -3 \\
\hline 4051 & -4 & 4651 & -4 \\
\hline 4101 & -4 & 4701 & -4 \\
\hline \hline Mean Point & -4 & & \\
\hline
\end{tabular}




\section{Chapter 5}

\section{Performance Analysis with}

\section{Environmental/System Mismatch}

Matched-field methods are developed to achieve performance improvements over the traditional plane wave beamforming methods by exploiting full field solutions to sound channel propagation. These full field solutions require an accurate environmental model as well as accurate system information, which are often unavailable in many practical applications. Instead, an environmental/system model is often assumed and some of the parameter values are assigned per one's best knowledge on the test site. The prior knowledge may come from on-site measurements, inversions, or derivations using empirical geoacoustic models. In the case with mismatch, i.e., the assumed environmental/system data differ from the actual physical conditions of an experiment, the detected pressure field will differ from the computed pressure field even at the true values of the parameters being estimated. As a result, the performance in parameter estimation is degraded even at high SNR. Sensitivity to mismatch is thus the most important liability with matched-field methods [8].

According to Ref. [8], environmental mismatch refers to uncertainty in the propagation model including sound velocity profile and bottom composition; system mismatch refers to errors in the receiving system including array shape and Doppler. ${ }^{1}$

\footnotetext{
${ }^{1}$ Statistical mismatch has also been discussed in Ref. [8], which refers to the need for covariance matrices in the design of adaptive processors, e.g., issues regarding the number of available snapshots.
} 
There have been numerous studies in analyzing the mismatch effects $[31,70,34,30,65]$ as well as reducing the sensitivity of matched-field methods to mismatch $[46,67,28] .{ }^{2}$ In general, the effects of mismatch include two aspects. First, it causes degradation in the output mainlobe peak. Second, it shifts the peak away from the correct position, leading to biased estimates. Most current research $[31,70,34,30]$ investigates, through simulations or some quantitative measures, how the peak output of a source localization algorithm is deteriorated in the presence of mismatch in individual environmental/system parameters. However, few results are reported regarding how a performance measure in parameter estimation (e.g., bias or mean square error) can be determined given the size of mismatch.

In this chapter, a modified Ziv-Zakai bound is proposed to incorporate mismatch into performance analysis.

\subsection{Modified Ziv-Zakai Bound}

From the view of parameter estimation, the environmental/system mismatch brings mismatch to the underlying data probability model. Let us first state the regular pdf by

$$
p\left(\mathbf{R}\left(\boldsymbol{\theta}, \mathcal{B}_{0}\right) ; \mathcal{M}\left(\boldsymbol{\theta}, \mathcal{B}_{0}\right)\right)
$$

where

$\mathbf{R}$ is any random observation vector which depends on a set of parameters, $\boldsymbol{\theta}$ and $\mathcal{B}_{0}$;

$\boldsymbol{\theta}$ is the parameter vector to be estimated;

$\mathcal{B}_{0}$ is any other parameter vector, deterministic or random, not included in $\boldsymbol{\theta}$ but necessary for specifying the pdf of $\mathbf{R}$; and

\footnotetext{
${ }^{2}$ In the context of source localization, one approach is joint estimation of the source location and the environmental/system parameters. This can be done in a Bayesian framework discussed in Chapter 3 and the Bayesian bounds can be applied. However, this approach soon becomes computationally expensive as the number of unknown parameters increases.
} 
$\mathcal{M}(\cdot)$ is a set of constant scalars, vectors, or matrices, directly defining the pdf. It is a function of the embedded parameters, $\boldsymbol{\theta}$ and $\mathcal{B}_{0}$, and can be constructed from the moments, for example, mean and covariance matrix.

For example, given $R$ is a real Gaussian variable with unknown mean $m_{0}$ but known variance $\sigma_{0}^{2}$, we have

$$
p\left(R\left(m_{0}, \sigma_{0}\right) ; \mathcal{M}\left(m_{0}, \sigma_{0}\right)\right)=\frac{1}{\sqrt{2 \pi} \sigma_{0}} \exp \left(\frac{\left(R-m_{0}\right)^{2}}{2 \sigma_{0}^{2}}\right) .
$$

Then $\theta=m_{0}, \mathcal{B}_{0}=\sigma_{0}$, and $\mathcal{M}(\cdot)=\left\{m_{0}, \sigma_{0}, \sigma_{0}^{2}\right\}$.

To describe a mismatched probability model, we introduce a so-called mismatched probability density function:

$$
p\left(\mathbf{R}\left(\boldsymbol{\theta}, \mathcal{B}_{0}\right) ; \mathcal{M}\left(\boldsymbol{\theta}, \mathcal{B}_{a}\right)\right)
$$

The mismatched pdf has the same form as the regular pdf but now $\mathcal{M}(\cdot)$ is a function of the assumed background parameter set $\mathcal{B}_{a}$, while the observation vector is, as always, a function of the true background parameter set $\mathcal{B}_{0}$. Clearly, $\mathcal{B}_{a}$ includes all the parameters assumed for the background model except those being estimated, and some of parameters in $\mathcal{B}_{a}$ could have the same values as in $\mathcal{B}_{0}$.

Following the example in (5.2), we now assume the variance is mismatched, given by $\sigma_{a}^{2}$. The mismatched pdf is then defined by

$$
p\left(R\left(m_{0}, \sigma_{0}\right) ; \mathcal{M}\left(m_{0}, \sigma_{a}\right)\right)=\frac{1}{\sqrt{2 \pi} \sigma_{a}} \exp \left(\frac{\left(R-m_{0}\right)^{2}}{2 \sigma_{a}^{2}}\right) .
$$

Thus, $\theta=m_{0}, \mathcal{B}_{0}=\sigma_{0}, \mathcal{B}_{a}=\sigma_{a}$, and $\mathcal{M}(\cdot)=\left\{m_{0}, \sigma_{a}, \sigma_{a}^{2}\right\}$.

Eq. (5.3) actually summarizes the data probability model often used in developing a parameter estimation algorithm, although it is often not explicitly specified that the practical observation vector behaves according to a different background parameter set. For example, the maximum likelihood estimator can be expressed as 


$$
\hat{\boldsymbol{\theta}}_{M L}\left(\mathbf{R}, \mathcal{B}_{a}\right)=\arg \max _{\boldsymbol{\theta}} \ln p\left(\mathbf{R}\left(\boldsymbol{\theta}, \mathcal{B}_{0}\right) ; \mathcal{M}\left(\boldsymbol{\theta}, \mathcal{B}_{a}\right)\right)
$$

Under mismatched background, the binary hypotheses test is again stated by

$$
\begin{aligned}
& H_{0}: \quad \boldsymbol{\theta}=\boldsymbol{\theta}_{0}, \\
& H_{1}: \quad \boldsymbol{\theta}=\boldsymbol{\theta}_{1} .
\end{aligned}
$$

The pdf's under the hypotheses $H_{0}$ and $H_{1}$ are

$$
p\left(\mathbf{R}\left(\boldsymbol{\theta}_{0}, \mathcal{B}_{0}\right) ; \mathcal{M}\left(\boldsymbol{\theta}_{0}, \mathcal{B}_{a}\right)\right)
$$

and

$$
p\left(\mathbf{R}\left(\boldsymbol{\theta}_{1}, \mathcal{B}_{0}\right) ; \mathcal{M}\left(\boldsymbol{\theta}_{1}, \mathcal{B}_{a}\right)\right)
$$

respectively.

A key point to help understanding is that one does not know the true background parameter set and uses an assumed background parameter set. Therefore, either the Bayes or Neyman-Pearson criterion [72] again leads us to an optimum test based on the likelihood ratio. To minimize the total probability of error, this likelihood ratio test is stated by

$$
l(\mathbf{R})=\ln \left[\frac{p\left(\mathbf{R}\left(\boldsymbol{\theta}_{1}, \mathcal{B}_{0}\right) ; \mathcal{M}\left(\boldsymbol{\theta}_{1}, \mathcal{B}_{a}\right)\right)}{p\left(\mathbf{R}\left(\boldsymbol{\theta}_{0}, \mathcal{B}_{0}\right) ; \mathcal{M}\left(\boldsymbol{\theta}_{0}, \mathcal{B}_{a}\right)\right)}\right] \underset{H_{0}}{\stackrel{H_{1}}{\gtrless}} 0
$$

Following the same procedure leading to the regular Ziv-Zakai bound [22, 13], we obtain a modified Ziv-Zakai bound, which is

$$
\epsilon^{2} \geq \frac{1}{2 \tau} \int_{0}^{2 \tau} \delta \cdot V\left(\int_{-\tau}^{\tau-\delta} P_{e-m i s}(\theta, \theta+\delta) \cdot d \theta\right) \cdot d \delta
$$


for a scalar parameter uniformly distributed on $[-\tau, \tau]$, and

$$
\mathbf{a}^{T} \Sigma \mathbf{a} \geq \int_{0}^{\infty} \delta \cdot V\left(\max _{\Delta: \mathbf{a}^{T} \Delta=\delta} \int_{\Theta} \min [p(\boldsymbol{\theta}), p(\boldsymbol{\theta}+\boldsymbol{\Delta})] \cdot P_{e-m i s}(\boldsymbol{\theta}, \boldsymbol{\theta}+\boldsymbol{\Delta}) d \boldsymbol{\theta}\right) \cdot d \delta
$$

for a vector parameter with prior distribution of $p(\boldsymbol{\theta})$. $P_{\boldsymbol{e}-m i s}(\boldsymbol{\theta}, \boldsymbol{\theta}+\boldsymbol{\Delta})$ is the minimum achievable probability of error associated with the mismatched likelihood ratio test in (5.9).

In general, when a closed-form error probability for the regular likelihood ratio test is available, the mismatched error probability is solved in a similar way, particularly when the likelihood ratio can be expressed in terms of the correlation between the observation vector and the computed replica (matched-filter). In that case, replacing the matched replica with the mismatched replica does not change the form of the statistics of the likelihood ratio.

Therefore, given the size of mismatch, $\mathcal{B}_{a}-\mathcal{B}_{0}$, the modified Ziv-Zakai bound can be used to evaluate the mean square error in parameter estimation. The concept of the mismatched pdf works well with the ZZB because the likelihood ratio test depends on both the observation and the assumed statistical information about the observation. This enables us to separate the observation and the assumed statistics in evaluating the error probability. In contrast, in deriving the BCRB or WWB, the observation vector is integrated out and the bound can only be expressed in terms of the assumed statistics.

\subsection{Application to the Matched-Field Problem}

Given the data model in (3.28), dependence on environmental/system parameters is embedded in the term of Green's function. ${ }^{3}$ Using the notation in Section 5.1, the

\footnotetext{
${ }^{3}$ The mismatch (including the system mismatch) has no effects to the white noise model, which is used by assuming a weak dependence of the noise upon the channel. However, if the colored (correlated) noise field is considered, the mismatch should be incorporated in the same way for the signal field.
} 
probability density function in (3.32) can be rewritten as

$$
\begin{aligned}
p\left(\mathbf{R}\left(\boldsymbol{\theta}, \mathcal{B}_{0}\right) ; \mathcal{M}\left(\boldsymbol{\theta}, \mathcal{B}_{0}\right)\right)= & \frac{1}{\left|\pi \mathrm{K}_{\mathbf{R}}\left(\boldsymbol{\theta}, \mathcal{B}_{0}\right)\right|} \exp \left(-\mathbf{R}^{\dagger}\left(\boldsymbol{\theta}, \mathcal{B}_{0}\right) \mathrm{K}_{\mathbf{R}}^{-1}\left(\boldsymbol{\theta}, \mathcal{B}_{0}\right) \mathbf{R}\left(\boldsymbol{\theta}, \mathcal{B}_{0}\right)\right) \\
= & \frac{1}{\prod_{m=1}^{M}\left|\pi \mathrm{K}_{\mathbf{R}}\left(f_{m}, \boldsymbol{\theta}, \mathcal{B}_{0}\right)\right|^{L}} \\
& \prod_{m=1}^{M} \prod_{l=1}^{L} \exp \left(-\mathbf{R}_{l}^{\dagger}\left(f_{m}, \boldsymbol{\theta}, \mathcal{B}_{0}\right) \mathrm{K}_{\mathbf{R}}^{-1}\left(f_{m}, \boldsymbol{\theta}, \mathcal{B}_{0}\right) \mathbf{R}_{l}\left(f_{m}, \boldsymbol{\theta}, \mathcal{B}_{0}\right)\right)
\end{aligned}
$$

The log likelihood ratio has the form

$$
l(\mathbf{R})=\sum_{m=1}^{M} \sum_{l=1}^{L}\left|\mathbf{R}_{l}^{\dagger}\left(f_{m}, \mathcal{B}_{0}\right) \mathbf{g}\left(f_{m}, \boldsymbol{\theta}_{1}, \mathcal{B}_{0}\right)\right|^{2}-\sum_{m=1}^{M} \sum_{l=1}^{L}\left|\mathbf{R}_{l}^{\dagger}\left(f_{m}, \mathcal{B}_{0}\right) \mathbf{g}\left(f_{m}, \boldsymbol{\theta}_{0}, \mathcal{B}_{0}\right)\right|^{2}
$$

and the associated error probability

$$
\begin{aligned}
P_{e}\left(\boldsymbol{\theta}_{1} \mid \boldsymbol{\theta}_{0}\right)= & \operatorname{Pr}\left(\sum_{m=1}^{M} \sum_{l=1}^{L}\left|\mathbf{R}_{l}^{\dagger}\left(f_{m}, \boldsymbol{\theta}_{0}, \mathcal{B}_{0}\right) \mathbf{g}\left(f_{m}, \boldsymbol{\theta}_{1}, \mathcal{B}_{0}\right)\right|^{2}\right. \\
& \left.\geq \sum_{m=1}^{M} \sum_{l=1}^{L}\left|\mathbf{R}_{l}^{\dagger}\left(f_{m}, \boldsymbol{\theta}_{0}, \mathcal{B}_{0}\right) \mathbf{g}\left(f_{m}, \boldsymbol{\theta}_{0}, \mathcal{B}_{0}\right)\right|^{2}\right)
\end{aligned}
$$

is solved in Appendix C.

Now we assume some mismatch in background parameters, so that $\mathcal{B}_{a}$ is different from $\mathcal{B}_{0}$. The mismatched pdf is available by modifying the parameter-dependence in (5.12), which is

$$
\begin{aligned}
p\left(\mathbf{R}\left(\boldsymbol{\theta}, \mathcal{B}_{0}\right) ; \mathcal{M}\left(\boldsymbol{\theta}, \mathcal{B}_{a}\right)\right)= & \frac{1}{\left|\pi \mathrm{K}_{\mathbf{R}}\left(\boldsymbol{\theta}, \mathcal{B}_{a}\right)\right|} \exp \left(-\mathbf{R}^{\dagger}\left(\boldsymbol{\theta}, \mathcal{B}_{0}\right) \mathrm{K}_{\mathbf{R}}^{-1}\left(\boldsymbol{\theta}, \mathcal{B}_{a}\right) \mathbf{R}\left(\boldsymbol{\theta}, \mathcal{B}_{0}\right)\right) \\
= & \frac{1}{\prod_{m=1}^{M}\left|\pi \mathrm{K}_{\mathbf{R}}\left(f_{m}, \boldsymbol{\theta}, \mathcal{B}_{a}\right)\right|^{L}} \\
& \prod_{m=1}^{M} \prod_{l=1}^{L} \exp \left(-\mathbf{R}_{l}^{\dagger}\left(f_{m}, \boldsymbol{\theta}, \mathcal{B}_{0}\right) \mathrm{K}_{\mathbf{R}}^{-1}\left(f_{m}, \boldsymbol{\theta}, \mathcal{B}_{a}\right) \mathbf{R}_{l}\left(f_{m}, \boldsymbol{\theta}, \mathcal{B}_{0}\right)\right)
\end{aligned}
$$


Under this pdf, the likelihood ratio test is stated by

$$
l(\mathbf{R})=\sum_{m=1}^{M} \sum_{l=1}^{L}\left|\mathbf{R}_{l}^{\dagger}\left(f_{m}, \mathcal{B}_{0}\right) \mathbf{g}\left(f_{m}, \boldsymbol{\theta}_{1}, \mathcal{B}_{a}\right)\right|^{2}-\sum_{m=1}^{M} \sum_{l=1}^{L}\left|\mathbf{R}_{l}^{\dagger}\left(f_{m}, \mathcal{B}_{0}\right) \mathbf{g}\left(f_{m}, \boldsymbol{\theta}_{0}, \mathcal{B}_{a}\right)\right|^{2} \underset{H_{0}}{\stackrel{H_{1}}{\gtrless}} 0 .
$$

Therefore, the minimum error probability associated with the mismatched likelihood ratio test involves

$$
\begin{aligned}
P_{e-m i s}\left(\boldsymbol{\theta}_{1} \mid \boldsymbol{\theta}_{0}\right)= & \operatorname{Pr}\left(\sum_{m=1}^{M} \sum_{l=1}^{L}\left|\mathbf{R}_{l}^{\dagger}\left(f_{m}, \boldsymbol{\theta}_{0}, \mathcal{B}_{0}\right) \mathbf{g}\left(f_{m}, \boldsymbol{\theta}_{1}, \mathcal{B}_{a}\right)\right|^{2}\right. \\
& \left.\geq \sum_{m=1}^{M} \sum_{l=1}^{L}\left|\mathbf{R}_{l}^{\dagger}\left(f_{m}, \boldsymbol{\theta}_{0}, \mathcal{B}_{0}\right) \mathrm{g}\left(f_{m}, \boldsymbol{\theta}_{0}, \mathcal{B}_{a}\right)\right|^{2}\right) .
\end{aligned}
$$

Substituting (5.17) into (5.10) or (5.11) properly, we are able to evaluate the modified Ziv-Zakai bound under the given background (environmental/system) mismatch, $\mathcal{B}_{a}-$ $\mathcal{B}_{0}$.

\subsubsection{Mismatched Error Probability}

The error probability in (5.17) has the same statistical structure as that in (5.14). They contain the same random components, $\mathbf{R}_{l}\left(f_{m}\right)$, and they both compare two sums of independent degree- $L$ complex Chi-squared variables, although with different weighting. Hence, (5.17) can be solved using the same approaches in Appendix C.

For example, for the single-frequency case, using Lindsey's approach, we only need to redefine $\tilde{y}_{l}$ and $\tilde{x}_{l}$ in (C.10) and (C.11) by

$$
\tilde{y}_{l}=\left[\mathbf{g}^{\dagger}\left(f_{0}, \boldsymbol{\theta}_{1}, \mathcal{B}_{a}\right) \mathbf{G}\left(f_{0}, \boldsymbol{\theta}_{0}, \mathcal{B}_{0}\right) \mathbf{g}^{\dagger}\left(f_{0}, \boldsymbol{\theta}_{1}, \mathcal{B}_{a}\right)\right]\left[\begin{array}{c}
\tilde{b}_{l}\left(f_{0}\right) \\
\mathbf{N}\left(f_{0}\right)
\end{array}\right]
$$

and

$$
\tilde{x}_{l}=\left[\begin{array}{ll}
\mathbf{g}^{\dagger}\left(f_{0}, \boldsymbol{\theta}_{0}, \mathcal{B}_{a}\right) \mathbf{G}\left(f_{0}, \boldsymbol{\theta}_{0}, \mathcal{B}_{0}\right) & \mathbf{g}^{\dagger}\left(f_{0}, \boldsymbol{\theta}_{0}, \mathcal{B}_{a}\right)
\end{array}\right]\left[\begin{array}{c}
\tilde{b}_{l}\left(f_{0}\right) \\
\mathbf{N}\left(f_{0}\right)
\end{array}\right]
$$


Again, both $\tilde{y}_{l}$ and $\tilde{x}_{l}$ are zero-mean complex Gaussian random variables. The difference is the covariance matrix of $\left[\tilde{y}_{l} \tilde{x}_{l}\right]^{T}$, which is now given by

$$
\begin{aligned}
\psi_{11} & =E\left[\tilde{y}_{l} \tilde{y}_{l}^{\dagger}\right] \\
& =\sigma_{b}^{2}\left(f_{0}\right) \cdot\left\|\mathbf{G}\left(f_{0}, \boldsymbol{\theta}_{0}, \mathcal{B}_{0}\right)\right\|^{2} \cdot\left|\mathbf{g}^{\dagger}\left(f_{0}, \boldsymbol{\theta}_{1}, \mathcal{B}_{a}\right) \mathbf{g}\left(f_{0}, \boldsymbol{\theta}_{0}, \mathcal{B}_{0}\right)\right|^{2}+\sigma_{n}^{2}\left(f_{0}\right), \\
\psi_{22} & =E\left[\tilde{x}_{l} \tilde{x}_{l}^{\dagger}\right] \\
& =\sigma_{b}^{2}\left(f_{0}\right) \cdot\left\|\mathbf{G}\left(f_{0}, \boldsymbol{\theta}_{0}, \mathcal{B}_{0}\right)\right\|^{2} \cdot\left|\mathbf{g}^{\dagger}\left(f_{0}, \boldsymbol{\theta}_{0}, \mathcal{B}_{a}\right) \mathbf{g}\left(f_{0}, \boldsymbol{\theta}_{0}, \mathcal{B}_{0}\right)\right|^{2}+\sigma_{n}^{2}\left(f_{0}\right),
\end{aligned}
$$

and

$$
\begin{aligned}
\psi_{12}= & \psi_{21}^{\dagger}=E\left[\tilde{y}_{l} \tilde{x}_{l}^{\dagger}\right] \\
= & \sigma_{b}^{2}\left(f_{0}\right) \cdot\left\|\mathbf{G}\left(f_{0}, \boldsymbol{\theta}_{0}, \mathcal{B}_{0}\right)\right\|^{2} \cdot \mathbf{g}^{\dagger}\left(f_{0}, \boldsymbol{\theta}_{1}, \mathcal{B}_{a}\right) \mathbf{g}\left(f_{0}, \boldsymbol{\theta}_{0}, \mathcal{B}_{0}\right) \mathbf{g}^{\dagger}\left(f_{0}, \boldsymbol{\theta}_{0}, \mathcal{B}_{0}\right) \mathbf{g}\left(f_{0}, \boldsymbol{\theta}_{0}, \mathcal{B}_{a}\right) \\
& +\sigma_{n}^{2}\left(f_{0}\right) \cdot \mathbf{g}^{\dagger}\left(f_{0}, \boldsymbol{\theta}_{1}, \mathcal{B}_{a}\right) \mathbf{g}\left(f_{0}, \boldsymbol{\theta}_{0}, \mathcal{B}_{a}\right) .
\end{aligned}
$$

Then the error probability has exactly the same equation in (C.48) but the related term definitions are based on the newly defined covariance matrix.

Similarly, the error probability can also be solved using Richmond's approach. It is still evaluated based on the statistics of the complex F-distribution (cf. (C.57) and (C.59)), but $\lambda_{1}$ and $\lambda_{2}$ in (C.57) are now the eigenvalues of the matrix

$$
\begin{aligned}
& \left(\mathbf{g}\left(f_{0}, \boldsymbol{\theta}_{1}, \mathcal{B}_{a}\right) \mathbf{g}^{\dagger}\left(f_{0}, \boldsymbol{\theta}_{1}, \mathcal{B}_{a}\right)-\mathbf{g}\left(f_{0}, \boldsymbol{\theta}_{0}, \mathcal{B}_{a}\right) \mathbf{g}^{\dagger}\left(f_{0}, \boldsymbol{\theta}_{0}, \mathcal{B}_{a}\right)\right) \\
& \left(\sigma_{b}^{2}\left(f_{0}\right) \mathbf{G}\left(f_{0}, \boldsymbol{\theta}_{0}, \mathcal{B}_{0}\right) \mathbf{G}^{\dagger}\left(f_{0}, \boldsymbol{\theta}_{0}, \mathcal{B}_{0}\right)+\sigma_{n}^{2}\left(f_{0}\right)\right)
\end{aligned}
$$

which can be solved following the same approach in Appendix C.1.2. Define

$$
\begin{gathered}
C\left(\boldsymbol{\theta}_{0} ; \mathcal{B}_{a}, \mathcal{B}_{0}\right)=\mathbf{g}^{\dagger}\left(f_{0}, \boldsymbol{\theta}_{0}, \mathcal{B}_{a}\right) \mathbf{g}\left(f_{0}, \boldsymbol{\theta}_{0}, \mathcal{B}_{0}\right), \\
C\left(\boldsymbol{\theta}_{0}, \boldsymbol{\theta}_{1} ; \mathcal{B}_{0}, \mathcal{B}_{a}\right)=\mathbf{g}^{\dagger}\left(f_{0}, \boldsymbol{\theta}_{0}, \mathcal{B}_{0}\right) \mathbf{g}\left(f_{0}, \boldsymbol{\theta}_{1}, \mathcal{B}_{a}\right),
\end{gathered}
$$


and

$$
C\left(\boldsymbol{\theta}_{1}, \boldsymbol{\theta}_{0} ; \mathcal{B}_{a}\right)=\mathbf{g}^{\dagger}\left(f_{0}, \boldsymbol{\theta}_{1}, \mathcal{B}_{a}\right) \mathbf{g}\left(f_{0}, \boldsymbol{\theta}_{0}, \mathcal{B}_{a}\right)
$$

Then the resulting quadratic equation of $\lambda$ is

$$
\lambda^{2}+A \cdot \lambda-B=0
$$

where

$$
A=\sigma_{b}^{2}\left(f_{0}\right) \cdot\left\|\mathbf{G}\left(f_{0}, \boldsymbol{\theta}_{0}, \mathcal{B}_{0}\right)\right\|^{2} \cdot\left(\left|C\left(\boldsymbol{\theta}_{0} ; \mathcal{B}_{a}, \mathcal{B}_{0}\right)\right|^{2}-\left|C\left(\boldsymbol{\theta}_{0}, \boldsymbol{\theta}_{1} ; \mathcal{B}_{0}, \mathcal{B}_{a}\right)\right|^{2}\right),
$$

and

$$
\begin{aligned}
B= & \sigma_{n}^{4}\left(f_{0}\right) \cdot\left(1-\left|C\left(\boldsymbol{\theta}_{1}, \boldsymbol{\theta}_{0} ; \mathcal{B}_{a}\right)\right|^{2}\right)+\sigma_{n}^{2}\left(f_{0}\right) \sigma_{b}^{2}\left(f_{0}\right) \cdot\left\|\mathbf{G}\left(f_{0}, \boldsymbol{\theta}_{0}, \mathcal{B}_{0}\right)\right\|^{2} \\
& \left(\left|C\left(\boldsymbol{\theta}_{0}, \boldsymbol{\theta}_{1} ; \mathcal{B}_{0}, \mathcal{B}_{a}\right)\right|^{2}+\left|C\left(\boldsymbol{\theta}_{0} ; \mathcal{B}_{a}, \mathcal{B}_{0}\right)\right|^{2}-\right. \\
& \left.2 \cdot \operatorname{Re}\left\{C\left(\boldsymbol{\theta}_{0} ; \mathcal{B}_{a}, \mathcal{B}_{0}\right) C\left(\boldsymbol{\theta}_{0}, \boldsymbol{\theta}_{1} ; \mathcal{B}_{0}, \mathcal{B}_{a}\right) C\left(\boldsymbol{\theta}_{1}, \boldsymbol{\theta}_{0} ; \mathcal{B}_{a}\right)\right\}\right) .
\end{aligned}
$$

It is easily shown that $B>0$ for $\boldsymbol{\theta}_{0} \neq \boldsymbol{\theta}_{1}$. So we have

$$
\lambda_{1}=\frac{1}{2} \cdot\left(-A-\sqrt{A^{2}+4 B}\right)<0,
$$

and

$$
\lambda_{2}=\frac{1}{2} \cdot\left(-A+\sqrt{A^{2}+4 B}\right)>0 .
$$

When multiple frequency components are used, the exact error probability is available based on the moment generating function of the log likelihood ratio (see Appendix C.2). As given in (C.80), the moment generating function is determined from the eigenvalues of the matrix in (C.75). With mismatched environment/system, this matrix is (5.23) with $f_{0}$ replaced by each $f_{m}$, and thus the eigenvalues at each 
frequency are solved using the procedure described above. However, the small-error approximation in Appendix C.2.2 is no longer useful since now the error probability can be close to one.

\subsubsection{Mismatch Analysis}

Calculations of the mismatched error probability involve three correlation terms of the received signal field in $(5.24),(5.25)$ and (5.26). The first one, $\left|C\left(\boldsymbol{\theta}_{0} ; \mathcal{B}_{a}, \mathcal{B}_{0}\right)\right|^{2}$, is

for the assumed and the true background parameter sets, with the parameter being estimated at the true position. This measures the peak degradation due to mismatch. The second one, $\left|C\left(\boldsymbol{\theta}_{0}, \boldsymbol{\theta}_{1} ; \mathcal{B}_{0}, \mathcal{B}_{a}\right)\right|^{2}$, is for the true, matched parameter set and the scanning, mismatched parameter set. As we later see, this could introduce peak shift. The third one, $\left|C\left(\boldsymbol{\theta}_{1}, \boldsymbol{\theta}_{0} ; \mathcal{B}_{a}\right)\right|^{2}$, corresponds to the true and the scanning parameter set both under mismatched background. This is similar to the regular ambiguity function in (4.21).

Recall from Section 4.3.3 that for perfectly known background, the error probability is inversely related to $\lambda_{R}$ for $\lambda_{R}>0$, and $\lambda_{R}(>1)$ itself is a function of the SNR and the field correlation. For a fixed SNR, the maximum error probability is achieved at the minimum $\lambda_{R}$, which corresponds to the maximum field correlation obtained by the closest mainlobe point. Under mismatched background, the situation is different.

When $\mathcal{B}_{a}-\mathcal{B}_{0}$ is small, although $\left|C\left(\boldsymbol{\theta}_{0} ; \mathcal{B}_{a}, \mathcal{B}_{0}\right)\right|^{2}$ is smaller than one (the peak value without mismatch), it is still the highest correlation value. In this case, the bias in parameter estimation can still be ignored but the mean square error would increase. As $\mathcal{B}_{a}-\mathcal{B}_{0}$ increases, $\left|C\left(\boldsymbol{\theta}_{0} ; \mathcal{B}_{a}, \mathcal{B}_{0}\right)\right|^{2}$ decreases further such that it is no longer the maximum correlation, i.e., at some $\boldsymbol{\theta}_{1}$, we have $\left|C\left(\boldsymbol{\theta}_{0} ; \mathcal{B}_{a}, \mathcal{B}_{0}\right)\right|^{2} \leq\left|C\left(\boldsymbol{\theta}_{0}, \boldsymbol{\theta}_{1} ; \mathcal{B}_{0}, \mathcal{B}_{a}\right)\right|^{2}$. In this case, the mismatched error probability is characterized by two operation regions.

The first region has $\left|C\left(\boldsymbol{\theta}_{0} ; \mathcal{B}_{a}, \mathcal{B}_{0}\right)\right|^{2}>\left|C\left(\boldsymbol{\theta}_{0}, \boldsymbol{\theta}_{1} ; \mathcal{B}_{0}, \mathcal{B}_{a}\right)\right|^{2}$, and thus $A>0$ and $\lambda_{R}>1$. This is similar to the mismatch-free case. For the single-frequency case, we have 


$$
\begin{aligned}
\lambda_{R} & =-\frac{\lambda_{1}}{\lambda_{2}} \\
& =\frac{1+\sqrt{1+\text { term }_{1}+\text { term }_{2}}}{-1+\sqrt{1+\text { term }_{1}+\text { term }_{2}}}
\end{aligned}
$$

where

$$
\begin{aligned}
\operatorname{term}_{1} & =4 \cdot \frac{1-\left|C\left(\boldsymbol{\theta}_{1}, \boldsymbol{\theta}_{0} ; \mathcal{B}_{a}\right)\right|^{2}}{S N R^{2}\left(f_{0}\right)\left(\left|C\left(\boldsymbol{\theta}_{0} ; \mathcal{B}_{a}, \mathcal{B}_{0}\right)\right|^{2}-\left|C\left(\boldsymbol{\theta}_{0}, \boldsymbol{\theta}_{1} ; \mathcal{B}_{0}, \mathcal{B}_{a}\right)\right|^{2}\right)^{2}} \\
\operatorname{term}_{2} & =4 \cdot \frac{\left|C\left(\boldsymbol{\theta}_{0} ; \mathcal{B}_{a}, \mathcal{B}_{0}\right)\right|^{2}+\left|C\left(\boldsymbol{\theta}_{0}, \boldsymbol{\theta}_{1} ; \mathcal{B}_{0}, \mathcal{B}_{a}\right)\right|^{2}-2 \cdot \text { term }_{0}}{S N R\left(f_{0}\right)\left(\left|C\left(\boldsymbol{\theta}_{0} ; \mathcal{B}_{a}, \mathcal{B}_{0}\right)\right|^{2}-\left|C\left(\boldsymbol{\theta}_{0}, \boldsymbol{\theta}_{1} ; \mathcal{B}_{0}, \mathcal{B}_{a}\right)\right|^{2}\right)^{2}}
\end{aligned}
$$

and

$$
\operatorname{term}_{0}=\operatorname{Re}\left[C\left(\boldsymbol{\theta}_{0} ; \mathcal{B}_{a}, \mathcal{B}_{0}\right) C\left(\boldsymbol{\theta}_{0}, \boldsymbol{\theta}_{1} ; \mathcal{B}_{0}, \mathcal{B}_{a}\right) C\left(\boldsymbol{\theta}_{1}, \boldsymbol{\theta}_{0} ; \mathcal{B}_{a}\right)\right]
$$

Obviously, the minimum $\lambda_{R}$ is one, which yields the maximum error probability of $1 / 2$ (cf. (4.22)). $\lambda_{R}=1$ is obtained when $\left|C\left(\boldsymbol{\theta}_{0} ; \mathcal{B}_{a}, \mathcal{B}_{0}\right)\right|^{2}=\left|C\left(\boldsymbol{\theta}_{0}, \boldsymbol{\theta}_{1} ; \mathcal{B}_{0}, \mathcal{B}_{a}\right)\right|^{2}$. This corresponds to another parameter point which cannot be resolved from the true parameter point by looking at correlation between the mismatched field and the true, mismatch-free field.

The second region is specified by $\left|C\left(\boldsymbol{\theta}_{0} ; \mathcal{B}_{a}, \mathcal{B}_{0}\right)\right|^{2}<\left|C\left(\boldsymbol{\theta}_{0}, \boldsymbol{\theta}_{1} ; \mathcal{B}_{0}, \mathcal{B}_{a}\right)\right|^{2}$. In this region, $A<0$ and $\left|\lambda_{1}\right|<\left|\lambda_{2}\right| . \lambda_{R}$ is then given by

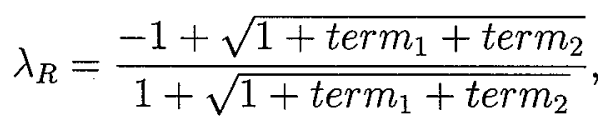

which is now smaller than one. The minimum value of $\lambda_{R}$ is actually zero, which is achieved at high SNR by some close mainlobe points whose mismatched fields are more correlated with the true, mismatch-free field $\left(\left|C\left(\boldsymbol{\theta}_{0}, \boldsymbol{\theta}_{1} ; \mathcal{B}_{0}, \mathcal{B}_{a}\right)\right|^{2}>\left|C\left(\boldsymbol{\theta}_{0} ; \mathcal{B}_{a}, \mathcal{B}_{0}\right)\right|^{2}\right)$ and also highly correlated with the mismatched field at the true parameter position $\left(\left|C\left(\boldsymbol{\theta}_{1}, \boldsymbol{\theta}_{0} ; \mathcal{B}_{a}\right)\right|^{2} \approx 1\right)$. From (4.22), $P_{e-m i s}\left(\boldsymbol{\theta}_{1} \mid \boldsymbol{\theta}_{0}\right)$ is one when $\lambda_{R}$ is zero. Thus the 
error probability falls between $1 / 2$ and 1 .

Fig. 5-1 presents some related field correlations for the range estimation problem in Section 4.1.1. The black line repeats the regular ambiguity function in Fig. 4-2(d). Note that in the original problem, the assumed sediment top wave-speed is 1572 $\mathrm{m} / \mathrm{s}$ and bottom wave-speed is $1593 \mathrm{~m} / \mathrm{s}$. The mismatch is introduced by assuming the true sediment top wave-speed at $1550 \mathrm{~m} / \mathrm{s}$ and bottom wave-speed at $1625 \mathrm{~m} / \mathrm{s}$. The red line is the correlation between the mismatched replica field at each scanning parameter point and the true signal field. A peak degradation (of about $0.4 \mathrm{~dB}$ ) at the true source position $\left(\theta_{0}=4125 \mathrm{~m}\right)$ is observed. Besides, at $\theta_{1} \approx 4135 \mathrm{~m}$, we have $C\left(\boldsymbol{\theta}_{0} ; \mathcal{B}_{a}, \mathcal{B}_{0}\right) \approx C\left(\boldsymbol{\theta}_{0}, \boldsymbol{\theta}_{1} ; \mathcal{B}_{0}, \mathcal{B}_{a}\right)$, and the highest correlation is now at about 4130 m.

The mismatched error probability is calculated at $\mathrm{SNR}=10 \mathrm{~dB}$. The result is shown in Fig. 5-2. Between $\theta_{1}=4135 \mathrm{~m}$ and $4125 \mathrm{~m}, P_{e-m i s}\left(\theta_{1} \mid \theta_{0}\right)$ is larger than one half and actually very close to one between the highest correlation point and $\theta_{0}$. Outside this region, $P_{e-m i s}\left(\theta_{1} \mid \theta_{0}\right)$ goes quickly down to zero, particularly on the left side of $\theta_{0}$.

Although these two operation regions demonstrate two almost opposite behaviors, they are subject to the same physical rule. That is, when the signal field at one parameter point $\theta$ is closer to the true signal field, the MLE outputs at other parameter points can hardly be beyond that at $\theta$, particularly with low-level noise. Between $\theta_{1}$ $=4135 \mathrm{~m}$ and $4125 \mathrm{~m}$, the signal field is alway more correlated with the true field compared to that at the true source range, and the error probability increases as SNR increases; outside this region, the mismatched field at the true source range is always more correlated with the true field compared to those at all other points, and the error probability increases as SNR decreases. This explains why the transition between two regions is so abrupt at high SNR; at low SNR, the transition is much smoother.

Fig. 5-2 tells us that when $\mathcal{B}_{a}-\mathcal{B}_{0}$ is large, errors around the incorrect correlation peak dominate the performance in the high SNR region. This introduces a mismatchdependent bias term in parameter estimation. We would expect a nearly constant 

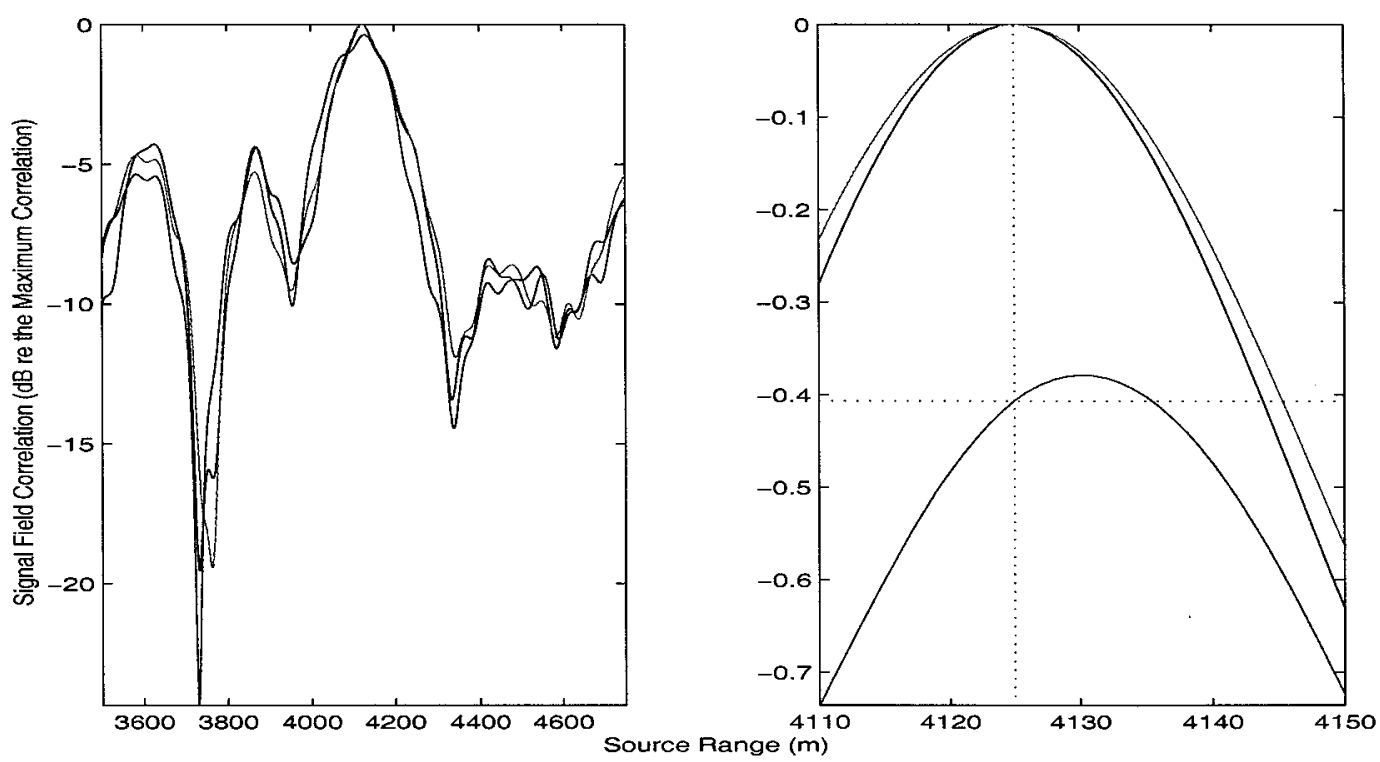

Figure 5-1: Signal ambiguity function for source range estimation: (4.21), mismatchfree (black line); (5.25), mismatched (red line); (5.26), mismatched (green line). The right panel shows the details around the true parameter region in which the vertical dotted line indicates the true source range position; the horizontal dotted line indicates the peak degradation.
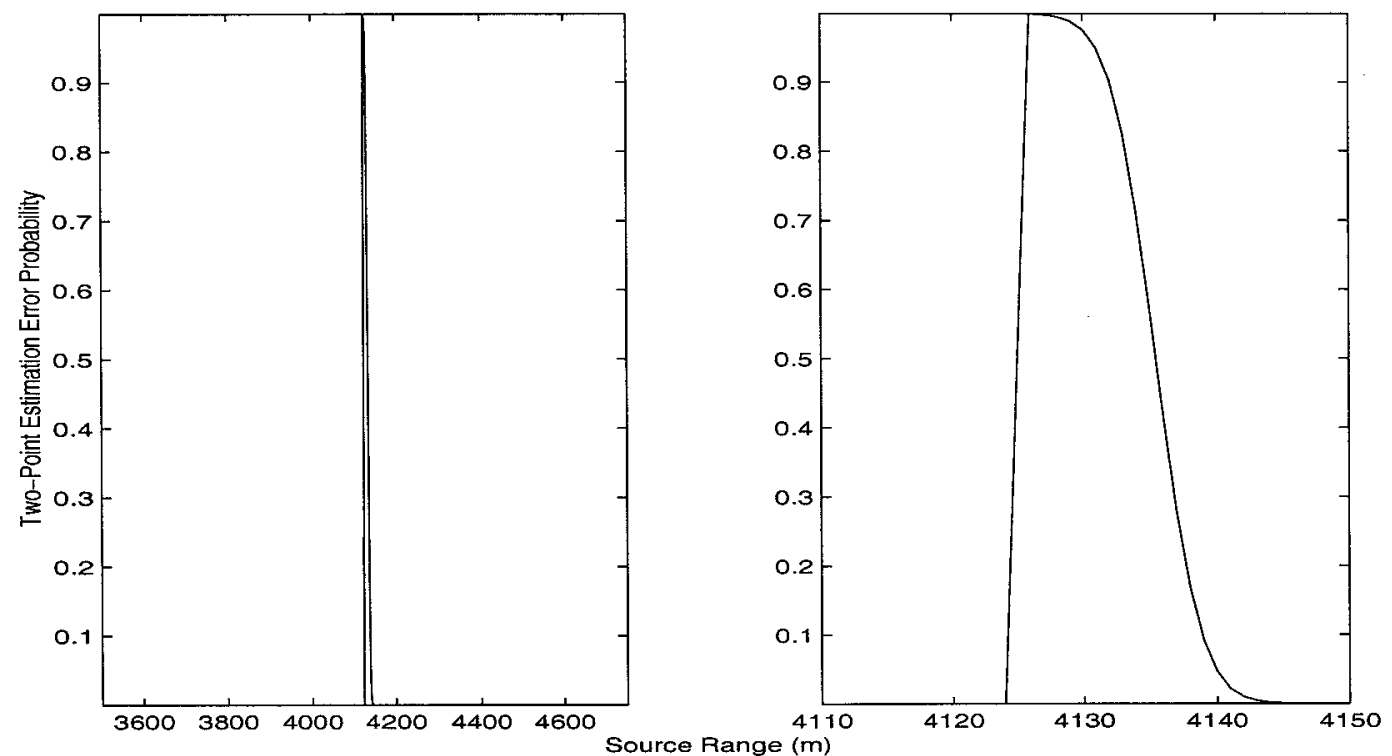

Figure 5-2: Two-point mismatched error probability for source range estimation. The right panel shows the details around the true parameter region. The true source range is at $4125 \mathrm{~m}$; the sensor-averaged SNR is $10 \mathrm{~dB}$. 
mean square error predicted by the modified Ziv-Zakai bound at high SNR, the squareroot of which gives an estimate of the bias. At low SNR, the sidelobe contributions through the terms of $C\left(\boldsymbol{\theta}_{0}, \boldsymbol{\theta}_{1} ; \mathcal{B}_{0}, \mathcal{B}_{a}\right)$ and $C\left(\boldsymbol{\theta}_{1}, \boldsymbol{\theta}_{0} ; \mathcal{B}_{a}\right)$ become important. It is interesting to note that to reduce the estimation error in the high SNR region, one may want to add some noise.

\subsection{Example: Mismatch Analysis in Bearing Esti- mation}

Consider the problem that a plane wave signal impinges on a vertical linear array of $N$ sensors as shown in Fig. 5-3. The sensors are uniformly spaced at $\Delta d$, and $\phi$ is the angle (from the horizontal line) of a plane wave arrival. To study the system mismatch, which is typical for traditional array problems, we choose the array tilt angle $\phi_{T}$ as the background parameter. Under this configuration, the parameter to be estimated is $u=\sin \left(\phi+\phi_{T}\right)$ ( $\phi_{T}$ is assumed known, although might be mismatched). We assume $u$ has a uniform distribution on $[-1,1]:{ }^{4}$

$$
p(u)=\frac{1}{2}, \quad|u|<1
$$

We further assume a narrowband source signal centered about a known frequency $f_{0}$. Similar to (3.28), the data model is stated by

$$
\mathbf{R}_{l}\left(f_{0}, u, \phi_{T}\right)=\tilde{b}_{l}\left(f_{0}\right) \mathbf{G}\left(f_{0}, u, \phi_{T}\right)+\mathbf{N}_{l}\left(f_{0}\right), \quad l=1, \ldots, L,
$$

where the signal field $\mathbf{G}(\cdot)$ is now

$$
\mathbf{G}\left(f_{0}, u, \phi_{T}\right)=\left[\begin{array}{llll}
1 & e^{-j \frac{2 \pi f_{0}}{c} u \Delta d} & \ldots & e^{-j \frac{2 \pi f_{0}}{c} u(N-1) \Delta d}
\end{array}\right]^{T}
$$

\footnotetext{
${ }^{4}$ In many applications, one may want to assume a uniform distribution for $\phi$, which results in a pdf of $1 /\left(\pi \sqrt{1-u^{2}}\right)$ for $u$. Choosing $u$ as the unknown parameter simplifies the performance analysis because the local performance is independent of $u$.
} 


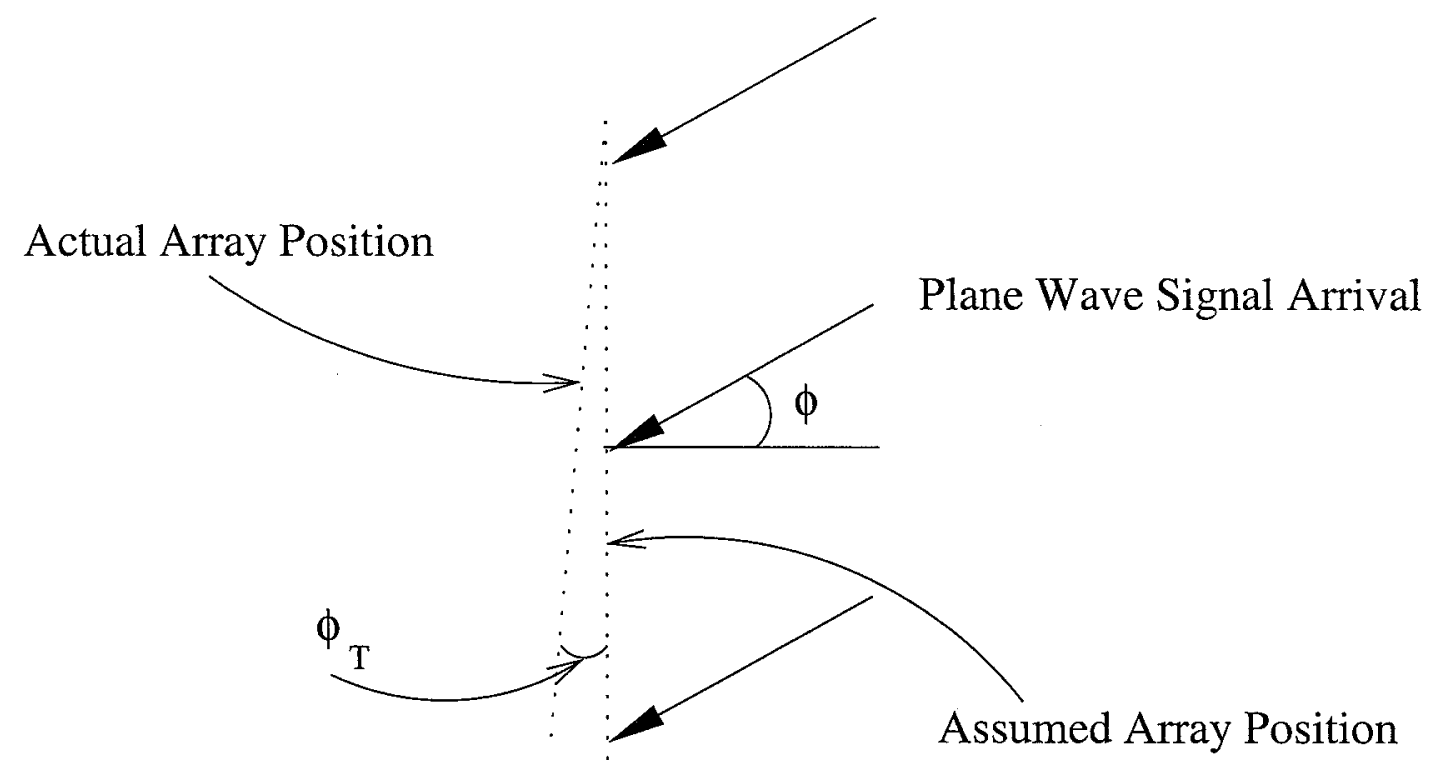

Figure 5-3: Uniform linear array. Actual and assumed array positions are also specified.

and $c$ is the wave propagation speed in the medium. Here we have used the first sensor as the reference sensor. The covariance matrix is again given by

$$
\mathrm{K}_{\mathbf{R}}\left(f_{0}, u, \phi_{T}\right)=\sigma_{b}^{2}\left(f_{0}\right) \mathbf{G}\left(f_{0}, u, \phi_{T}\right) \mathbf{G}^{\dagger}\left(f_{0}, u, \phi_{T}\right)+\sigma_{n}^{2}\left(f_{0}\right) \mathrm{I}
$$

For this plane wave signal model, it is easily shown that the ambiguity function has a nice form:

$$
\begin{aligned}
\Gamma\left(f_{0}, u_{0}, u_{1} ; \phi_{T}\right) & =\left|\left(\frac{\mathbf{G}\left(f_{0}, u_{0}, \phi_{T}\right)}{\left\|\mathbf{G}\left(f_{0}, u_{0}, \phi_{T}\right)\right\|}\right)^{\dagger}\left(\frac{\mathbf{G}\left(f_{0}, u_{1}, \phi_{T}\right)}{\left\|\mathbf{G}\left(f_{0}, u_{1}, \phi_{T}\right)\right\|}\right)\right|^{2} \\
& =\left(\frac{\operatorname{sinc}\left[\frac{2 \pi f_{0}}{c} \cdot \frac{N \Delta d}{2} \cdot\left(u_{1}-u_{0}\right)\right]}{\operatorname{sinc}\left[\frac{2 \pi f_{0}}{c} \cdot \frac{\Delta d}{2} \cdot\left(u_{1}-u_{0}\right)\right]}\right)^{2}
\end{aligned}
$$

To avoid the grating lobes (poles of $(5.41)$ ), one often choose $\Delta d$ smaller than halfwavelength, $\lambda / 2$. So the ambiguity sidelobes occur at $\delta=u_{1}-u_{0}=\frac{2 k-1}{2} \cdot \frac{\lambda}{N \Delta d}$, where $k$ is an integer. Fig. 5-4 displays an example given the true bearing at $30^{\circ}$.

As we see in (5.41), the ambiguity function is independent of $u$. Note that we have $\left\|\mathbf{G}\left(f_{0}, u, \phi_{T}\right)\right\|^{2}=N$, so the signal-to-noise ratio defined by 


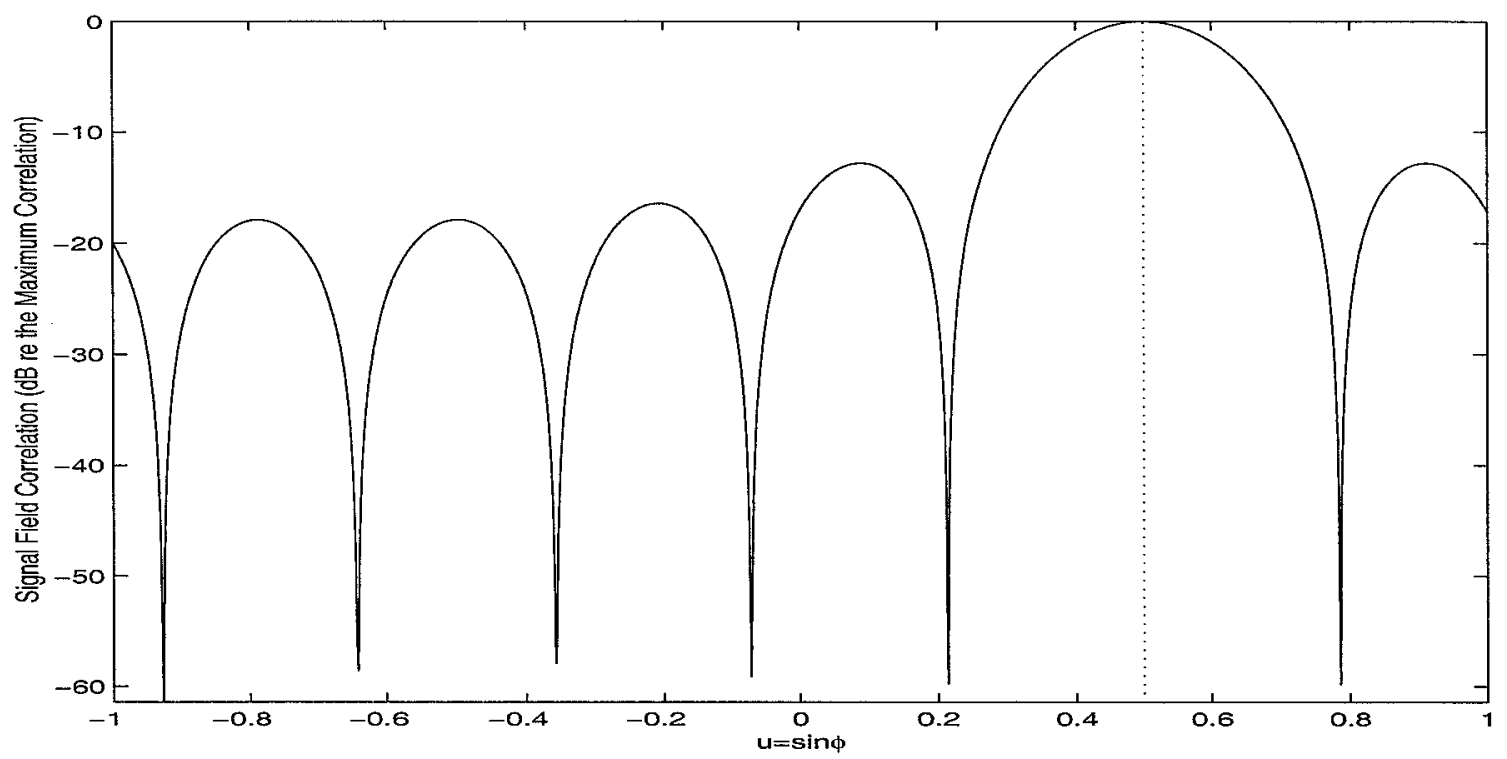

Figure 5-4: Signal ambiguity function for bearing estimation. The vertical line corresponds to the true bearing.

$$
\operatorname{SNR}\left(f_{0}\right)=\frac{N \sigma_{b}^{2}\left(f_{0}\right)}{\sigma_{n}^{2}\left(f_{0}\right)}
$$

is also independent of $u$. This greatly simplifies the evaluation of a global performance bound.

Ref. [14] has applied the ZZB to a similar bearing estimation problem. The approximation in (3.26) is used for the minimum probability of error, which is actually a lower bound for the time-delay/bearing estimation problem [77]. The optimum choice of $s$ is $1 / 2$, so we have

$$
P_{e}(u, u+\delta) \approx e^{\mu\left(\frac{1}{2} ; \delta\right)+\frac{1}{8} \ddot{\mu}\left(\frac{1}{2} ; \delta\right)} \cdot \operatorname{erfc}\left(\frac{1}{2} \sqrt{\ddot{\mu}\left(\frac{1}{2} ; \delta\right)}\right)
$$

where $\mu\left(\frac{1}{2} ; \delta\right)$ and $\ddot{\mu}\left(\frac{1}{2} ; \delta\right)$ are given by $[14]$ :

$$
\mu\left(\frac{1}{2} ; \delta\right)=-L \ln \left(1+\frac{A}{4}(1-C(\delta))\right)
$$


and

$$
\ddot{\mu}\left(\frac{1}{2} ; \delta\right)=L \frac{2 A(1-C(\delta))}{1+\frac{A}{4}(1-C(\delta))},
$$

respectively, with

$$
A=\frac{S N R^{2}\left(f_{0}\right)}{1+S N R\left(f_{0}\right)}
$$

and $C(\delta)=\Gamma\left(f_{0}, u, u+\delta\right) . L$ is the number of snapshots.

The exact minimum error probability is also available based on the results in Appendix C. For example, one can substitute the signal-to-noise ratio in (5.42) and the ambiguity function in (5.41) into (4.23) and then (4.22). To verify the tightness of the error approximation in (5.43), we evaluate the ZZB for the mismatch-free case, i.e., the actual array position is the assumed vertical position ( $\phi_{T}=0$ in Fig. 5-3). For this example, we choose $\Delta d=\frac{7}{16} \lambda, N=8$, and $L=10$.

Fig. 5-5 shows the evaluation results, in which three operation regions described in Fig. 4-21 are observed. The approximation to error probability is very close to the exact one such that the resulting bounds are hardly distinguishable. They both follow the mean square errors computed from the MLE simulations well, and the predicted threshold SNR is about $-3 \mathrm{~dB}$. Ambiguity analysis developed in Section 4.4 can also be applied, and the results for the true bearing at $30^{\circ}$ are shown in Fig. 5-6. The threshold SNR at this local parameter point is about $-2 \mathrm{~dB}$.

We then assume there is some mismatch between the assumed and the actual array positions through the array tilt angle $\phi_{T}$ (cf. Fig. 5-3). In this case, $\mathcal{B}_{0}=\phi_{T}$ and $\mathcal{B}_{a}=0$. The related field correlations are given by

$$
\begin{gathered}
C\left(u_{1}, u_{T 0} ; \mathcal{B}_{a}, \mathcal{B}_{0}\right)=e^{j \frac{2 \pi f_{0}}{c} \cdot \frac{(N-1) \Delta d}{2} \cdot\left(u_{1}-u_{T 0}\right)} \cdot \frac{\operatorname{sinc}\left[\frac{2 \pi f_{0}}{c} \cdot \frac{N \Delta d}{2} \cdot\left(u_{1}-u_{T 0}\right)\right]}{\operatorname{sinc}\left[\frac{2 \pi f_{0}}{c} \cdot \frac{\Delta d}{2} \cdot\left(u_{1}-u_{T 0}\right)\right]}, \\
C\left(u_{1}, u_{0} ; \mathcal{B}_{a}\right)=e^{j \frac{2 \pi f_{0}}{c} \cdot \frac{(N-1) \Delta d}{2} \cdot\left(u_{1}-u_{0}\right)} \cdot \frac{\operatorname{sinc}\left[\frac{2 \pi f_{0}}{c} \cdot \frac{N \Delta d}{2} \cdot\left(u_{1}-u_{0}\right)\right]}{\operatorname{sinc}\left[\frac{2 \pi f_{0}}{c} \cdot \frac{\Delta d}{2} \cdot\left(u_{1}-u_{0}\right)\right]}
\end{gathered}
$$




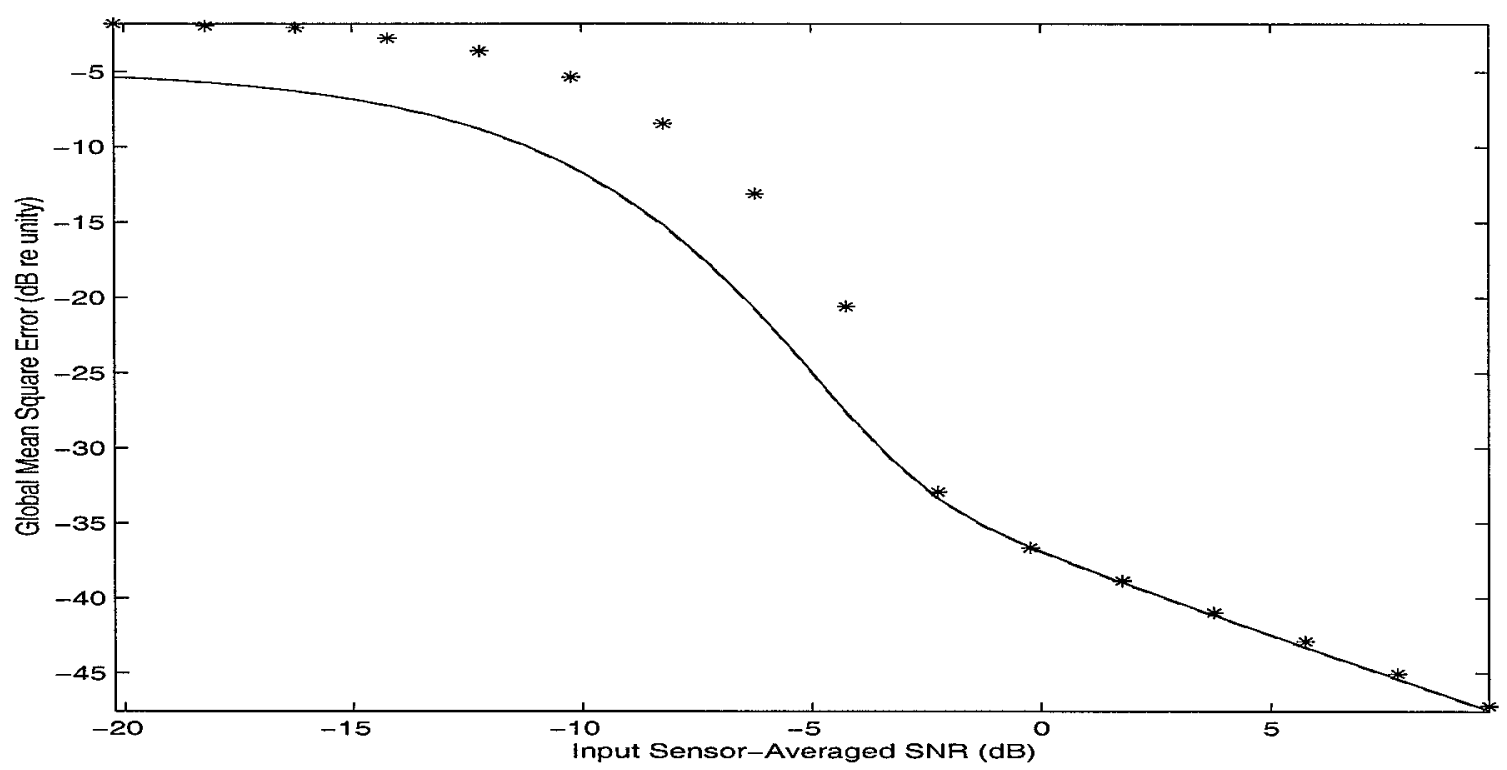

Figure 5-5: Bearing estimation mean square error given by the ZZB with exact (solid line) or lower-bounded (dashed line) minimum error probability, and the MLE simulations $(*)$. No mismatch is assumed.

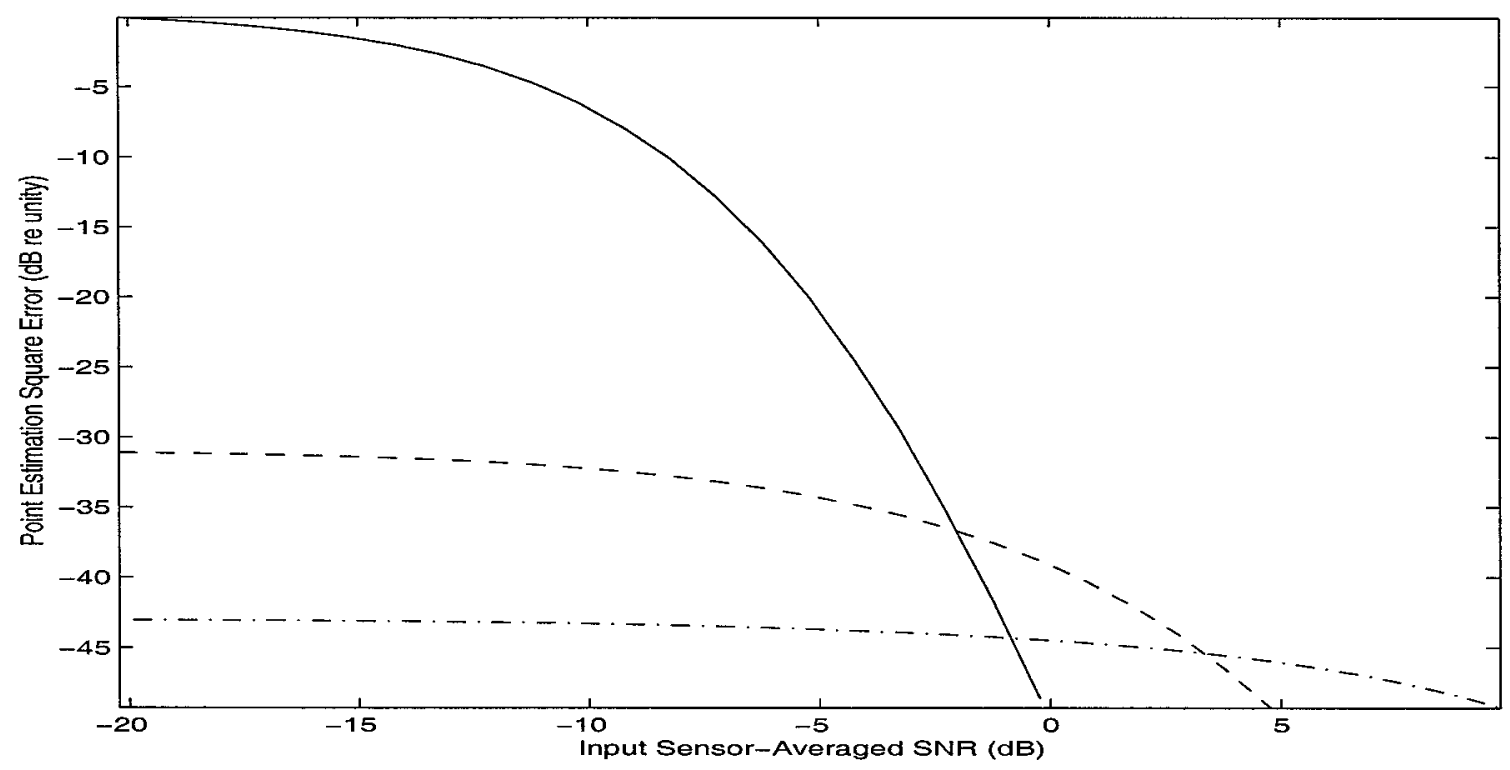

Figure 5-6: Probabilistic square errors as a function of SNR at different ambiguity points: close mainlobe point (0.49, dashdot line); distant mainlobe point (0.54, dashed line); and distant sidelobe point $\left(-1.0\right.$, solid line). The true $u$ is at $0.5\left(\phi=30^{\circ}\right)$. 


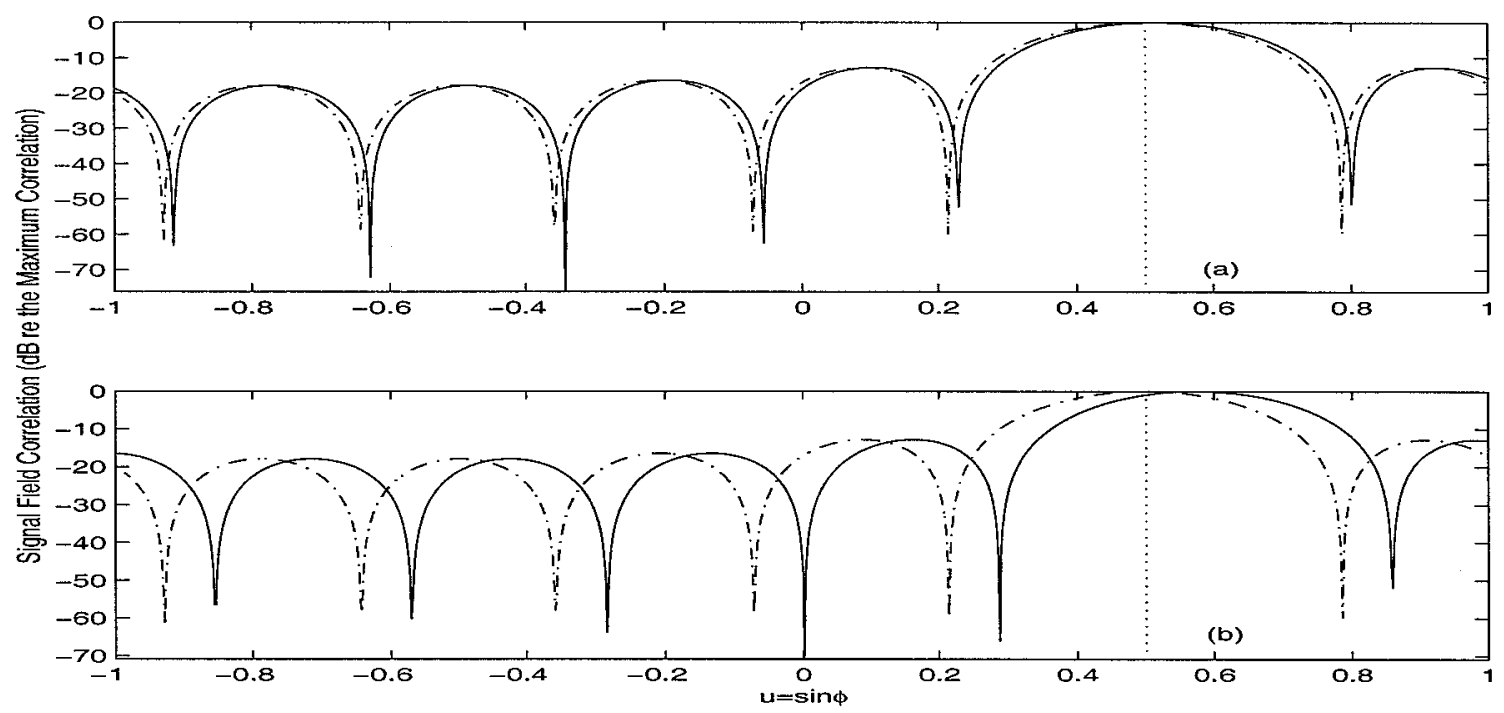

Figure 5-7: Mismatched ambiguity function for bearing estimation: (a) $\phi_{T}=1^{\circ}$; (b) $\phi_{T}=5^{\circ}$. Solid line is for (5.47); dashdot line is for (5.48); the vertical line corresponds to the true bearing.

where $u_{0}=\sin \phi_{0}, u_{1}=\sin \phi_{1}$, and $u_{T 0}=\sin \left(\phi_{0}+\phi_{T}\right)$. Some examples are given in Fig. 5-7 for $\phi_{T}=1^{\circ}$ and $5^{\circ}$, respectively, with the true bearing at $30^{\circ}(u=0.5)$.

The peak degradation, $\left|C\left(u_{0}, u_{T 0} ; \mathcal{B}_{a}, \mathcal{B}_{0}\right)\right|^{2}$, is $(5.47)$ evaluated at $u_{1}=u_{0}$. Obviously, it is no longer the ambiguity mainlobe peak. Instead, the mismatched mainlobe peak occurs at $u_{1}=u_{T 0}$, i.e., $\phi_{1}=\phi_{0}+\phi_{T}$. Substituting these correlation terms to (5.32) or (5.36) and then to (4.22) and (5.10), we can evaluate the modified Ziv-Zakai bound for bearing estimation with system mismatch.

Figs. 5-8 and 5-9 give the results for $\phi_{T}=1^{\circ}$ and $\phi_{T}=5^{\circ}$, respectively. We see that as $\phi_{T}$ increases, the bound is nearly independent of the SNR at high SNR, and is actually dominated by the square of the mismatch-introduced bias. This bias can be approximately computed using the bound. Note that at high SNR the bearing estimation variance in the mismatched case is similar to that without mismatch. Then we have

$$
|\mathrm{Bias}|=\sqrt{\mathrm{MZZB}\left(\mathrm{SNR}_{0}\right)-\mathrm{ZZB}\left(\mathrm{SNR}_{0}\right)},
$$

where $\mathrm{SNR}_{0}$ is in the high SNR region. The sign of the bias can be determined from 


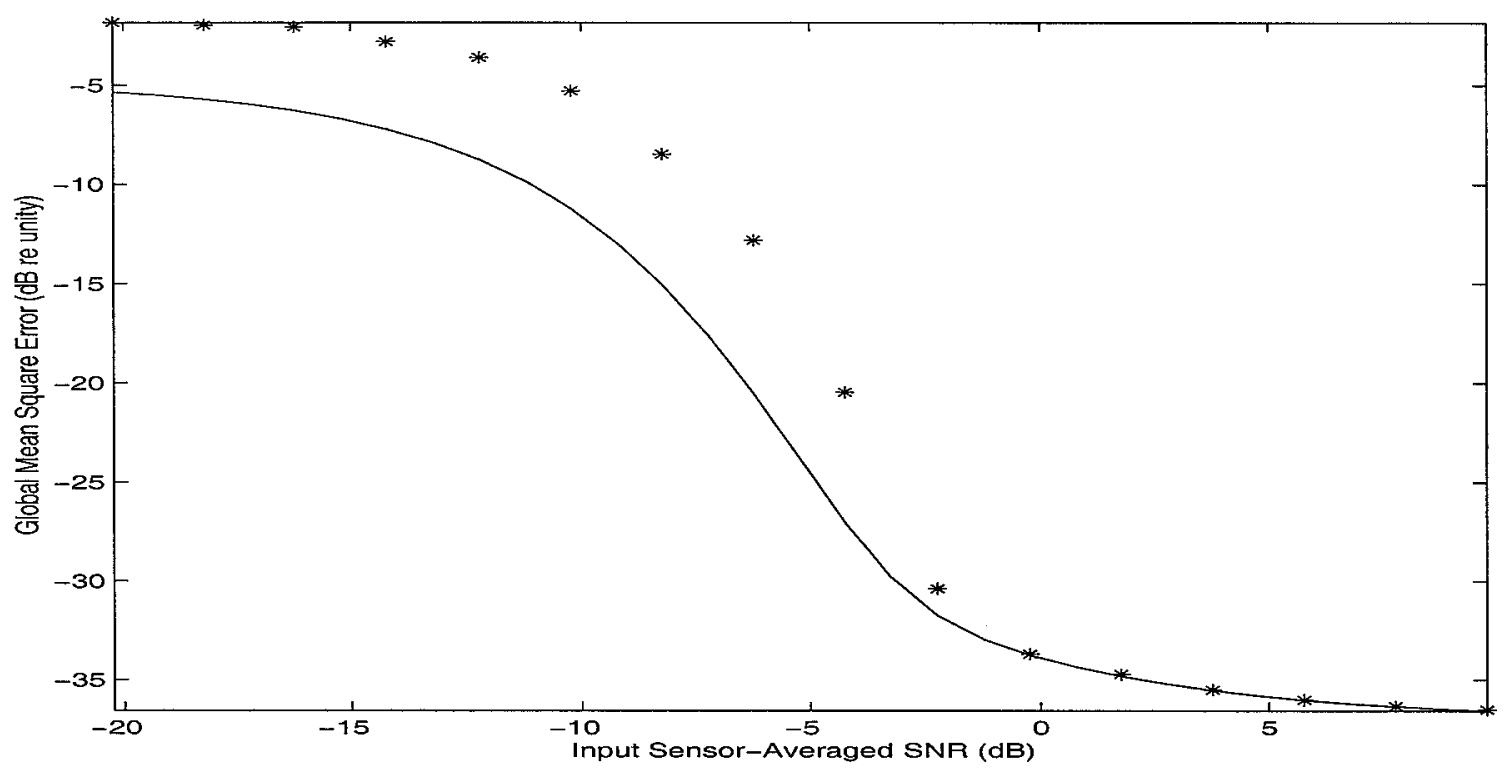

Figure 5-8: Bearing estimation mean square error given by the modified ZZB (solid line), and the MLE simulations $\left({ }^{*}\right)$. The array tilt angle is mismatched by $1^{\circ}$.

the position of the mismatched mainlobe peak. Using this approach, for $\phi_{T}=1^{\circ}$, the predicted bias is about 0.0142 ; for $\phi_{T}=5^{\circ}$, this is 0.0712 . Note that $u$ is a dimensionless quantity.

The above predictions can be verified in this simple problem. Note that at each $\phi$, the bias in estimation of $u$ is given by

$$
\sin \left(\phi+\phi_{T}\right)-\sin (\phi) \approx \phi_{T} \cos (\phi)=\phi_{T} \sqrt{1-u^{2}}
$$

for small $\phi_{T}$. Ignoring the effects around the endfire region, the averaged bias is given by

$$
\operatorname{Bias} \approx \int_{-1}^{1} \phi_{T} \sqrt{1-u^{2}} p(u) d u
$$

In this example, the estimated bias is 0.0137 for $\phi_{T}=1^{\circ}$, or 0.0685 for $\phi_{T}=5^{\circ}$. 


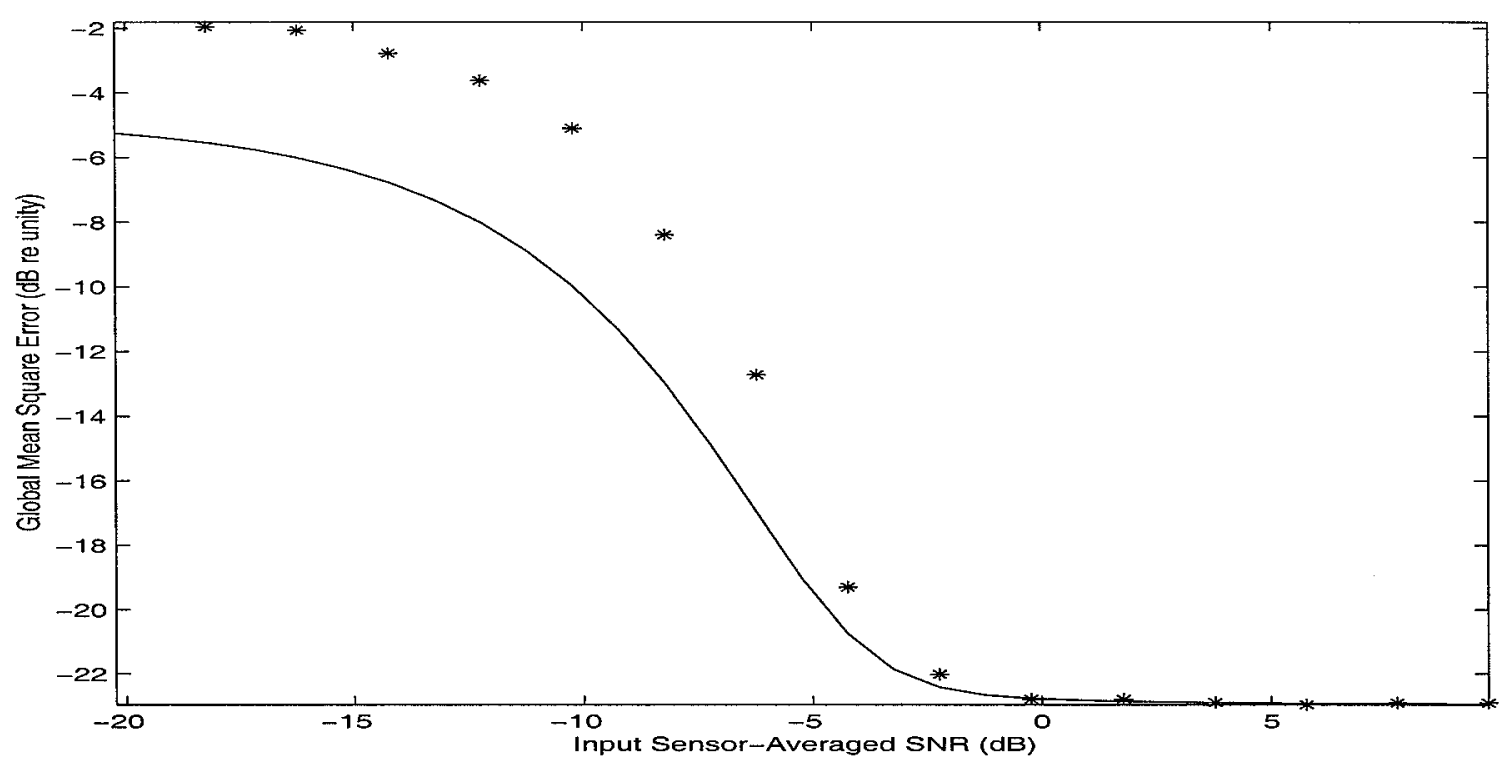

Figure 5-9: Bearing estimation mean square error given by the modified ZZB (solid line), and the MLE simulations $(*)$. The array tilt angle is mismatched by $5^{\circ}$.

\subsection{Summary}

In this chapter, a modified Ziv-Zakai bound is proposed to bound the mean square error of parameter estimation using incorrect environmental/system parameter set. The modified bound can be evaluated using the modified two-point estimation error probability derived under mismatched circumstances. This mismatched error probability can be applied to analyze the ambiguity in the presence of system/environmental mismatch and helps to understand the introduced bias at high SNR levels.

Application to bearing estimation shows that the modified bound gives quite close performance predictions to the mismatched MLE, including the mean square error in all SNR regions and the bias at high SNR. This makes the bound a useful tool for performance analysis in practical sonar/radar applications. For example, given the environmental/system uncertainty, we may evaluate the effects of possible mismatch for an assumed environmental/system parameter set.

We should point out that the mismatch discussed in this section is the deterministic mismatch. Further developments should be possible to include stochastic mismatch. 


\section{Chapter 6}

\section{Examples}

In this chapter, we present some examples to verify the developed theoretical performance predictions and investigate some typical performance behaviors in matchedfield parameter estimation. We start with some discussions on the computational aspects in bound's evaluation, and then present the evaluation results of the BCRB, WWB and ZZB for source localization and estimation of environmental parameters in a shallow water waveguide. These results are analyzed using the ambiguity analysis developed in Chapter 4, and compared to the MLE simulations; some of them are compared to the real data processing.

The environment chosen is one of the range-independent tracks of the SWellEX-3 experiments $[17,58]$ and the geoacoustic model [68] in Fig. 4-1 is used for simulations. Unless otherwise stated, we consider a single frequency component at $101 \mathrm{~Hz}$. The sensor spacing is $7.5 \mathrm{~m}$, equal to the half-wavelength at this frequency; 16 sensors are used spanning the water column from $78 \mathrm{~m}$ to $190 \mathrm{~m}$. To be consistent with the processing described in Ref. [17], except for the single-snapshot example, ten independent snapshots are available for each estimate. Therefore, as given in Appendix D, the output SNR is the input SNR plus $17 \mathrm{~dB}$.

We mainly concern source localization in the presence of environmental uncertainty or mismatch. Some other relevant issues, for example, snapshot-deficiency and incoherent frequency averaging, are addressed as well. In all examples, the KRAKEN normal modes model [56] is used to calculate the replica field for the given environ- 
mental and source-receiver configuration.

\subsection{Computational Issues}

Section 3.2 suggests that a closed-form expression is not available for any of the Bayesian bounds in the matched-field problem. As a result, we have to implement the bound's evaluation numerically and thus the numerical accuracy and the computational load have to be balanced, which is discussed in this section.

\subsubsection{Bayesian Cramer-Rao Bound}

In evaluation of the BCRB, one needs to take the derivative of the Green's function with respect to the parameter studied. This can be done numerically using a finite difference for the parameter perturbation, which for the scalar parameter case is

$$
\frac{\partial}{\partial \theta} \mathbf{G}\left(f_{0}, \theta\right) \approx \frac{\mathbf{G}\left(f_{0}, \theta+\Delta \theta\right)-\mathbf{G}\left(f_{0}, \theta-\Delta \theta\right)}{2 \Delta \theta}
$$

The parameter perturbation $\Delta \theta$ is chosen so that the Green's function is about linear with respect to the perturbation, and thus the derivative is well approximated by this numerical method. The linearity condition can be stated by

$$
T=\frac{\mathbf{G}\left(f_{0}, \theta+\Delta \theta\right)-\mathbf{G}\left(f_{0}, \theta\right)}{\mathbf{G}\left(f_{0}, \theta\right)-\mathbf{G}\left(f_{0}, \theta-\Delta \theta\right)} \approx 1
$$

which means $|T| \approx 1$ and $\angle T \approx 0$.

Since the derivative of $p(\boldsymbol{\theta})$ is involved in (3.12), using a uniform parameter pdf is inappropriate, whose first-order derivative has impulses at both ends. A frequentlyused trick is adding some smoothing function at both ends without changing much 
the original pdf. For example,

$$
p\left(\theta_{i}\right)=\left\{\begin{aligned}
\frac{1}{2 \tau_{i}\left(1+\alpha_{i}\right)}\left(1+\cos \frac{\theta_{i}+\alpha_{i} \tau_{i}}{\tau_{i}\left(1-\alpha_{i}\right)} \pi\right), & -\tau_{i} \leq \theta_{i} \leq-\alpha_{i} \tau_{i}, \\
\frac{1}{\tau_{i}\left(1+\alpha_{i}\right)}, & -\alpha_{i} \tau_{i}<\theta_{i}<\alpha_{i} \tau_{i}, \\
\frac{1}{2 \tau_{i}\left(1+\alpha_{i}\right)}\left(1+\cos \frac{\theta_{i}-\alpha_{i} \tau_{i}}{\tau_{i}\left(1-\alpha_{i}\right)} \pi\right), & \alpha_{i} \tau_{i} \leq \theta_{i} \leq \tau_{i} .
\end{aligned}\right.
$$

For this choice of $p\left(\theta_{i}\right)$, the pdf itself and its first-order derivative are continuous across the end points. Given that the parameters are independent with each other, the Fisher information due to the a priori parameter distribution is a diagonal matrix and the diagonal component corresponds to the contribution from each individual parameter, which is for the pdf in (6.3)

$$
\left[\mathrm{J}_{A}\right]_{i i}=\frac{\pi^{2}}{\tau_{i}^{2}\left(1-\alpha_{i}^{2}\right)}
$$

Unless otherwise stated, evaluation of the BCRB and WWB assumes this modified uniform parameter distribution with $\alpha_{i}=0.95$.

\subsubsection{Weiss-Weinstein Bound}

In evaluating the one-test point WWB, we need to determine a possible set of test points, from which the optimum test point is sought at each SNR. As discussed in Section 4.3.2, the optimum test point best captures the ambiguity behavior at each SNR. Therefore, at high SNR, only the test points close to zero need to be considered. Furthermore, due to the sensitivity of the WWB to small test points, those test points have to be finely sampled. On the contrary, in the transition region, the sidelobe points or distant mainlobe points dominate the bound's evaluation and the test point set can be more coarsely sampled due to the effect of the increasing noise level, as long as it still cover the entire ambiguity space.

In the multiple-parameter case, the choice of the test vectors is more complicated. A general approach is separating a multi-dimensional test vector into multiple onedimensional test points. However, this could lead to a less tight bound if the error coupling between any two parameters is significant. The multi-dimensional ambiguity 
function implies the error coupling in parameter estimation and thus is used to choose the set of test vectors.

It is worth noting that for a multiple-test point WWB or multiple-parameter WWB, maximization over all the test points requires remarkable computational effort even though the matched-field problem doesn't show a uniform, strongly-oscillatory ambiguity structure and thus may only require a small number of test points. An alternative (and more efficient) approach is choosing a reasonably large dense set of test points distributed over the a priori region. Once the number of test point is large enough, it is not necessary to maximize over their positions. Nonetheless, one must be careful in choosing the test point set because the matrix $\mathbf{Q}$ in the bound's evaluation (cf. (3.18)) could be singular when the number of test points is large. This can be mathematically verified based on the property derived in Ref. [29, Appendix A].

\subsubsection{Ziv-Zakai Bound}

To define a sample set of parameter perturbations (and thus a path of integration in (3.23)), the approach for choosing the test point set of the WWB can be applied as well. The valley-filling operation in Fig. 3-1 is shown to increase the bound significantly for time-delay/bearing estimation. This improvement, however, can often be ignored for matched-field parameter estimation because the ambiguity property is not uniform across the parameter interval and some strong periodic structures are averaged out.

For the multiple-parameter ZZB, the constraint in (3.24), $\mathbf{a}^{T} \boldsymbol{\Delta}=\delta$, doesn't uniquely determine $\Delta$ but forces $\Delta$ to have the following form:

$$
\Delta=\frac{\mathbf{a}}{\mathbf{a}^{T} \mathbf{a}} \delta+\mathbf{b}
$$

with $\mathbf{a}^{T} \mathbf{b}=0$. So the maximization in (3.24) is implemented within the $\left(N_{p}-\right.$ 1)-dimensional space. The function to be maximized (error probability associated with a perturbation averaged over the parameter space) can be a complicated (e.g., 
multi-modal) function of the free parameters depending on the ambiguity structure in multi-dimensional space. This is a well-posed optimization problem, so a local (gradient-based) or global (simulated annealing or genetic algorithm) optimization algorithm [23] is desired to improve the computational efficiency.

\subsubsection{Computational Load}

In this section, we give the order of the required computations in evaluation of each bound based on some typical implementations.

Note that a numerical integration over the prior parameter space is required for any of three Bayesian bounds (cf. (3.12), (3.44), (3.23), and (3.24)). In general, the parameter sampling step is chosen so that at most parameter points the field is linear

with respect to the parameter perturbation equal to the sampling step. This sampling step is also the minimum test point for the WWB or the minimum perturbation for the ZZB. To simplify the programming, one would use this sampling step as the numerical integration step. However, this approach soon becomes computationally intolerable as the dimensionality of the parameter space increases. In fact, although the integrand of each integration, as given in (3.33), (3.43), or (3.45), is parameterdependent in the matched-field problem, it is usually weakly parameter-dependent. Accordingly, the numerical integration can be implemented on much coarser grids, e.g., eight times of the sampling step.

Consider a problem with a single frequency component and three parameters. Recall that $L$ is the number of snapshots and $N$ is the number of sensors. Some other related quantities are denoted by

$N P_{1}$ : number of discrete points for Parameter 1

$N P_{2}$ : number of discrete points for Parameter 2;

$N P_{3}$ : number of discrete points for Parameter 3;

$N I_{1}$ : number of integration points for Parameter 1 ;

$N I_{2}$ : number of integration points for Parameter 2; 
Table 6.1: Computational load in evaluation of performance bound at one SNR. Also given are the results in performance simulations of the MLE and MMSE.

\begin{tabular}{|l||r|c|}
\hline Algorithm & Order of the Required Computations & Comments \\
\hline \hline BCRB & $120 \cdot N \cdot N I_{1} \cdot N I_{2} \cdot N I_{3}$ & \\
\hline WWB & $1.5 \cdot N H^{2} \cdot N \cdot N I_{1} \cdot N I_{2} \cdot N I_{3}$ & No optimization \\
\hline ZZB & $15 \cdot N D \cdot L \cdot N \cdot N I_{1} \cdot N I_{2} \cdot N I_{3}$ & Local searching \\
\hline MLE & $10 \cdot N T_{1} \cdot L \cdot N \cdot N P_{1} \cdot N P_{2} \cdot N P_{3}$ & \\
\hline MMSE & $40 \cdot N T_{2} \cdot L \cdot N \cdot N P_{1} \cdot N P_{2} \cdot N P_{3}$ & \\
\hline
\end{tabular}

$\mathrm{NI}_{3}$ : number of integration points for Parameter 3;

$N H:$ number of test points in evaluation of the WWB; and

$N D$ : number of perturbation points in evaluation of the ZZB.

Table 6.1 gives the required computations at one SNR in terms of the number of real addition/multiplication operations. We see that the BCRB is most computationally efficient; the WWB and the ZZB require similar computational effort for typical choices of $N H, N D$ and $L$, which, however, is far significant compared to the BCRB.

The results in performance simulations of the MLE and MMSE (Minimum Mean Square Error Estimator, also called Conditional Mean Estimator [43]) are also listed. We denote $N T_{1}$ as the number of the total Monte Carlo trials in the MLE simulation, and $N T_{2}$ in the MMSE simulation. To obtain robust performance results, $N T_{i}$ should be on the order of $N P_{1} \cdot N P_{2} \cdot N P_{3}$. This makes the simulation computationally prohibitive. One has to choose a small $N T_{i}$, and thus the simulated performance is hardly exact. Even so, both simulations are still more computationally expensive because we often have $N P_{i} \gg N I_{i}$. This may explain why the performance bound is of more interest. Finally, by choosing $N T_{2}=N T_{1}$, the computational load in the MMSE simulation is about four times of that in the MLE simulation.

We should point out that as the number of parameters increases, the required runtime memory and data storage soon also become the limiting factors in computation. 


\subsection{Source Range Estimation with Perfectly Known Environment}

The scenario examined is part of the track A in SWellEX-3 [17], in which the source depth is nearly a constant at $60 \mathrm{~m}$, and the source range varies from $3500 \mathrm{~m}$ to 4750 $\mathrm{m}$. We are to determine the average performance of source range estimation over this interval. For the purposes of demonstration, we first consider a scalar case, i.e., source range estimation with perfectly known environment. In later sections, some environmental uncertainties or mismatches are incorporated as well.

We assume the source range is uniformly distributed over the interval of [3500 $4750] \mathrm{m}$. The source depth is fixed at $60 \mathrm{~m}$ and the environmental parameters are those specified in Fig. 4-1. Results of the linearity test suggest a perturbation of 0.5 $\mathrm{m}$ for source range is small enough to maintain the field linearity. This is also used as the minimum test point of the WWB and the starting point of the integration path of the ZZB. Fig. 6-1 shows the global mean square error specified by the BCRB, WWB and ZZB. Among them, the multiple-test point WWB uses a set of pre-selected test points around the mainlobe and sidelobes without optimization. For comparison, the MLE simulation results are also presented on the basis of 5000 Monte Carlo trials at each SNR.

At high SNR, the WWB (one-test point or multiple-test point) approaches the BCRB, but they both are a bit $(<1 \mathrm{~dB})$ lower than the MLE simulations. This is because the local performance is not uniform across the assumed parameter interval, as shown in Fig. 6-2, although in this case the variation is not significant. In contrast, the ZZB exactly follows the MLE asymptotic performance. It degrades at very high SNR due to the numerical problem (Using a smaller minimum parameter sampling improves the bound).

Below SNR = -1 dB, MLE error increases significantly demonstrating the threshold phenomenon. Obviously, the BCRB is no longer useful in this region, while both the WWB and ZZB predict this threshold behavior. Note that in the asymptotic region, 


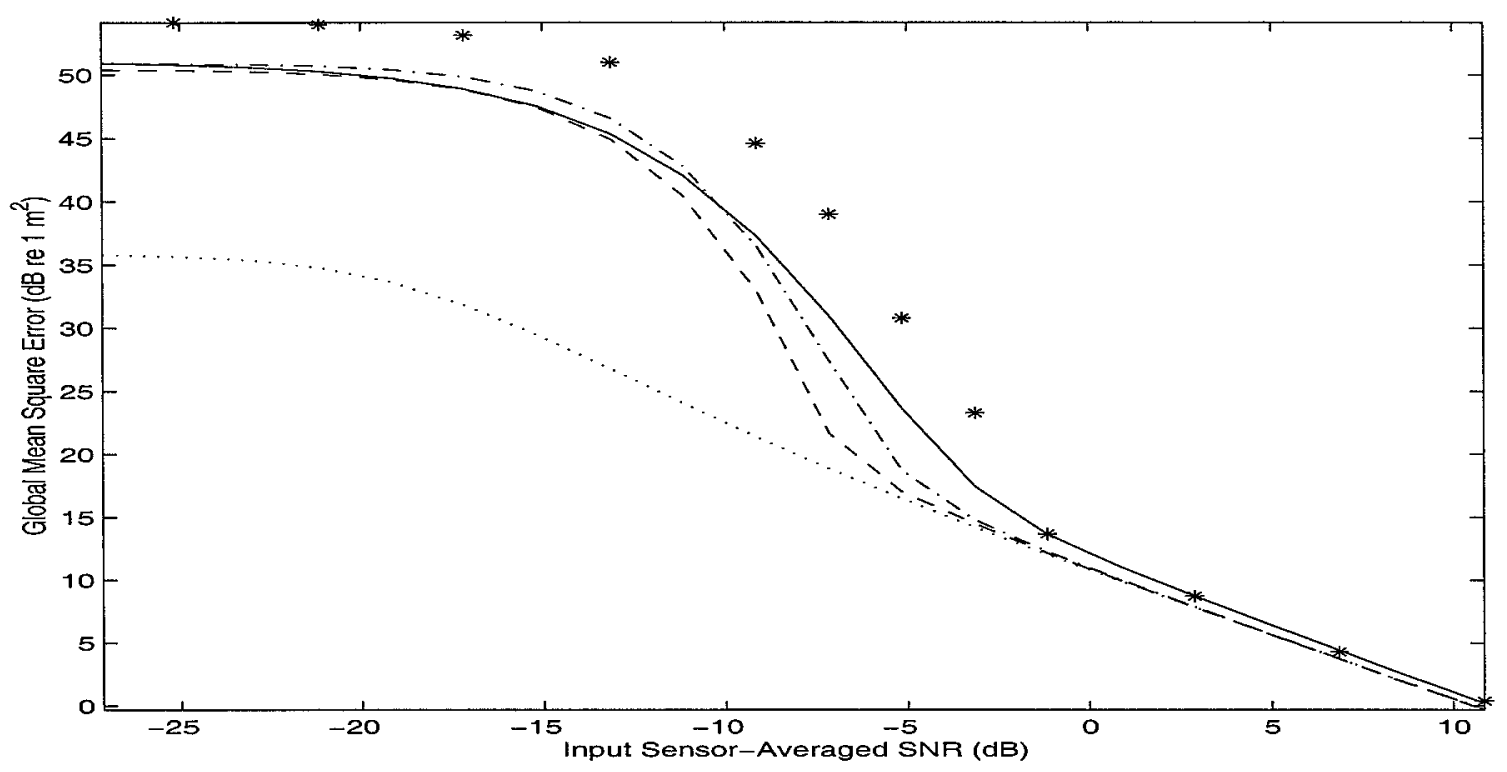

Figure 6-1: Performance bound evaluation for source range estimation. Solid line: ZZB; dashdot line: multiple-test point WWB; dashed line: one-test point WWB; dotted line: BCRB; and *: MLE simulations.

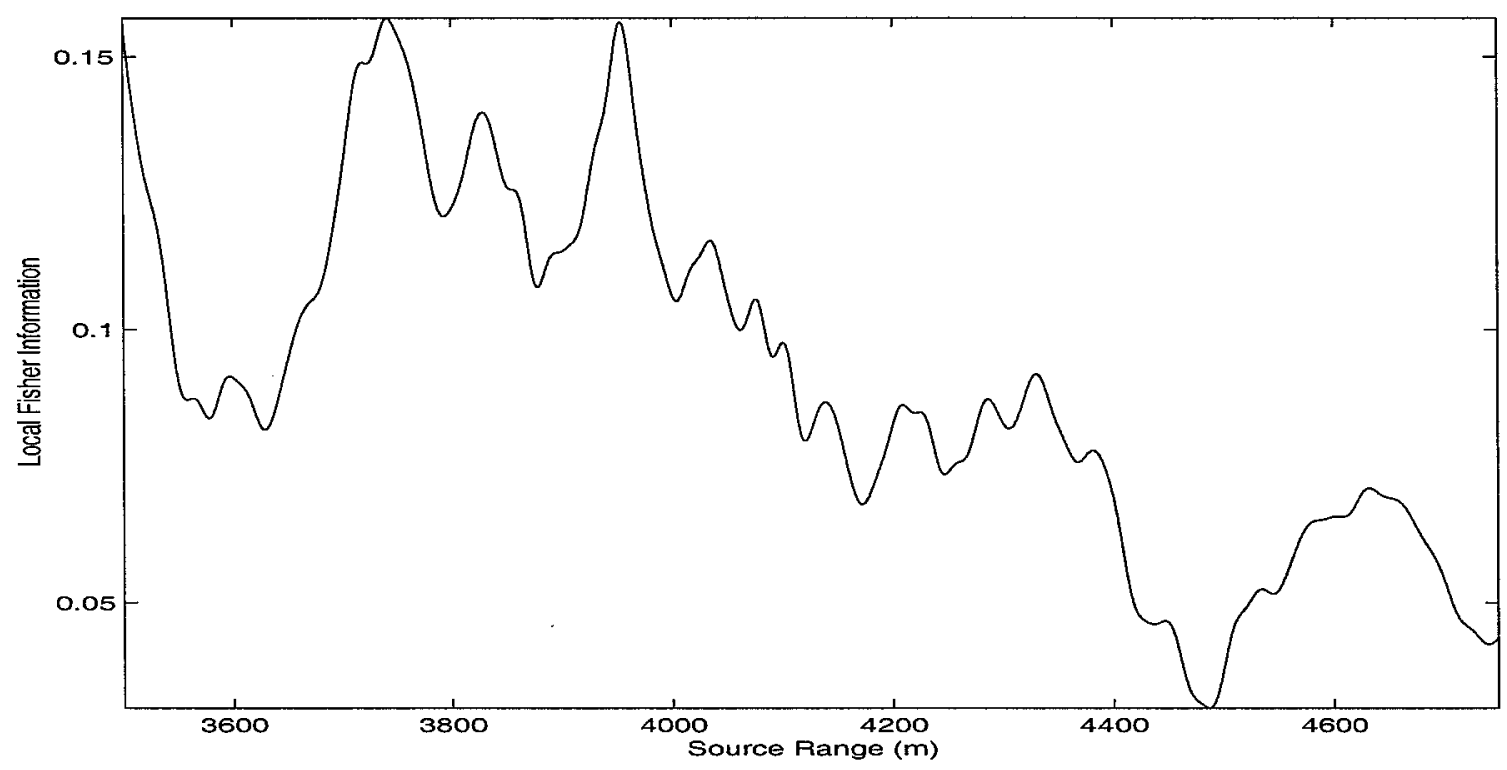

Figure 6-2: Local Fisher information for source range estimation. Sensor-averaged SNR is $10 \mathrm{~dB}$. 
the WWB and the ZZB can be expressed via a linear relation to SNR, i.e.,

$$
\text { WWB }(\mathrm{dB})=10.5-\mathrm{SNR}(\mathrm{dB})
$$

and

$$
\mathrm{ZZB}(\mathrm{dB})=11.2-\mathrm{SNR}(\mathrm{dB})
$$

According to the definition in (4.34), the predicted threshold SNR's are -6 dB (multipletest point $\mathrm{WWB})$ and $-3 \mathrm{~dB}(\mathrm{ZZB})$, respectively. Above $\mathrm{SNR}=-10 \mathrm{~dB}$, the $\mathrm{ZZB}$ is tighter (up to $5 \mathrm{~dB}$ higher) than the WWB. At very low SNR, both the WWB and ZZB are close to the variance of the a priori parameter distribution, which can be achieved by a conditional mean estimator [43]. The threshold SNR from the transition region to the no-information region is $-13 \mathrm{~dB}$ (predicted by the WWB; $-14 \mathrm{~dB}$ by the ZZB). Overall, for most SNR levels of practical interest, the ZZB gives the best performance prediction among three Bayesian bounds. ${ }^{1}$

Below the threshold SNR, the MLE MSE is always (up to $8 \mathrm{~dB}$ ) higher than the best prediction. This discrepancy can be explained by noting that in the low SNR region the optimal estimator is the conditional mean estimator rather than the MLE. For example, at very low SNR, observation is dominated by noise; in this case, the conditional mean estimate is the mean of the a priori parameter distribution, denoted by $\bar{\theta}$. We then have for the mean square error of the MLE

$$
E\left[\left(\hat{\theta}_{M L}-\theta\right)^{2}\right]=E\left[\left(\hat{\theta}_{M L}-\bar{\theta}\right)^{2}\right]+E\left[(\bar{\theta}-\theta)^{2}\right] .
$$

The second term of the right-hand-side is the mean square error of the conditional mean estimate (i.e., the variance of the a priori parameter distribution). The first term of the right-hand-side is always greater than zero. Obviously, in this region, the

\footnotetext{
${ }^{1}$ We also notice that below some SNR level the WWB is tighter than the ZZB. This has been observed in some other applications [14]. To explain this (and then obtain a uniform best performance bound), one may want to establish a connection between the Weiss-Weinstein-type bound and the Ziv-Zakai-type bound. This problem is not solved yet but Ref. [13] has made some trials.
} 


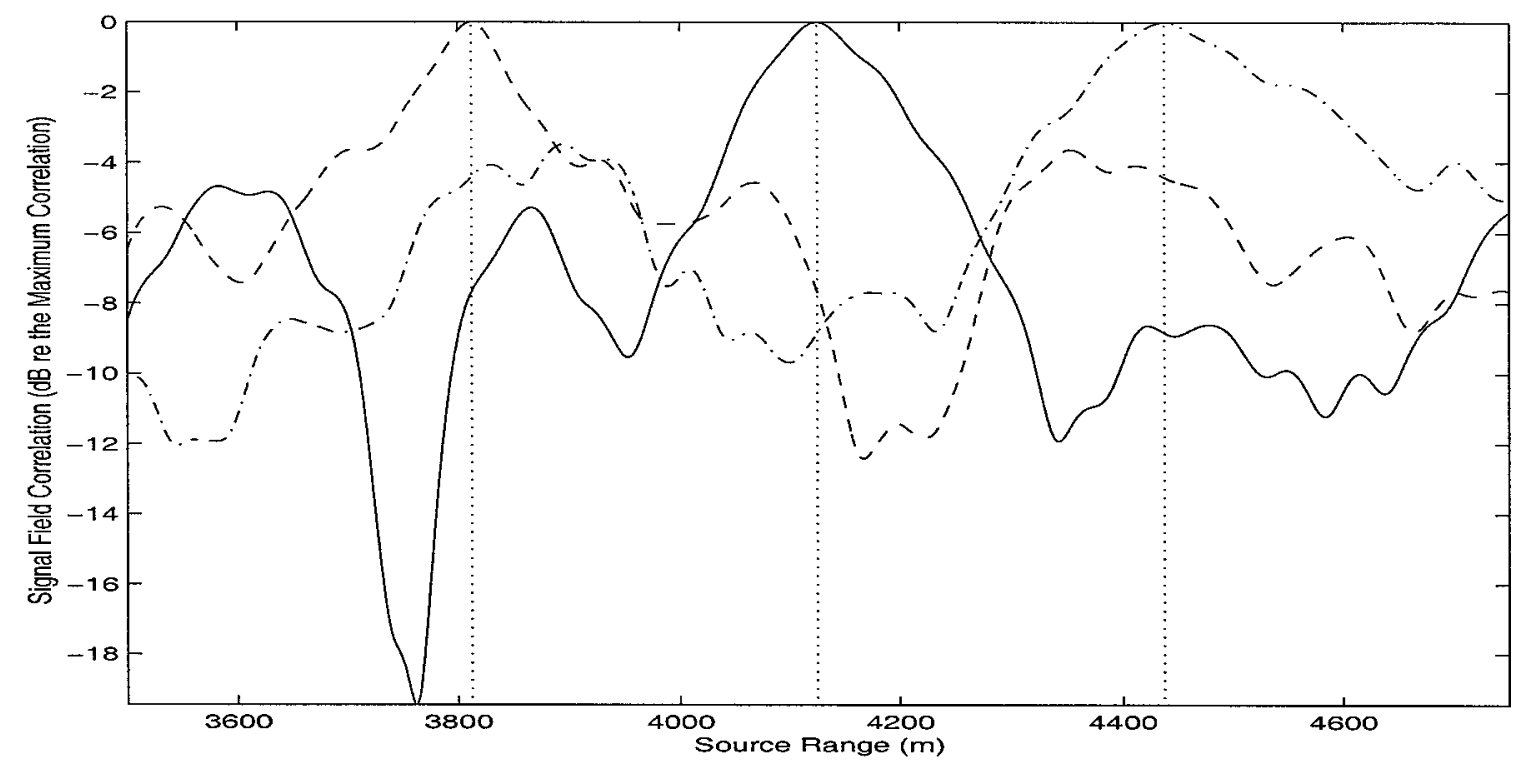

Figure 6-3: Example signal ambiguity functions for source range estimation at different true source range positions: (a) $3812 \mathrm{~m}$ (dashed line); (b) $4125 \mathrm{~m}$ (solid line); and (c) $4438 \mathrm{~m}$ (dashdot line). The dotted lines indicate individual true source range positions.

MLE has a larger mean square error than the conditional mean estimator and is no longer achievable by any fundamental performance bound.

Although the multiple-test point WWB does increase the bound particularly around the threshold region, evaluation of the one-test point WWB indicates the dominating ambiguity behavior through the optimal test point: close to zero at high SNR; average dominating sidelobe point in the transition region. Fig. 6-3 shows some ambiguity functions at different true range positions. Clearly, all those plots are characterized by a multi-modal structure. Accordingly, the optimal test points in the transition region fall between $500 \mathrm{~m}$ and $620 \mathrm{~m}$, which correspond to some high-level, distant sidelobes as seen from Fig. 6-3.

In Section 4.4.1, we have applied the two-point estimation error probability to analyze the effects of different ambiguity components of the surface. Given a true source range, the error probabilities and the probabilistic square errors associated with a close mainlobe point, a distant mainlobe point and a sidelobe point are evaluated using the error expression in (3.45). Comparing Fig. 4-18 to Fig. 6-1, we find that the results of error analysis are consistent with the bound's behavior. Indeed, as 
mentioned in Section 4.5.1, the predicted SNR is $-3 \mathrm{~dB}$ using the mean point of threshold predictions at 24 uniformly spaced parameter points or $-2 \mathrm{~dB}$ using the 83-percentile point. The latter one better describes the MLE simulation results.

\subsubsection{Number of Snapshots}

Number of independent snapshots plays a similar role as the signal-to-noise ratio. To obtain an asymptotic performance for parameter estimation, one may increase either the signal-to-noise ratio or the number of snapshots. As mentioned in Section 3.2.1, number of snapshots is determined by the observation time and the correlation time of the signal process [50]. This is true under the condition that the signal process is stationary during the entire observation period. Therefore, for a short-term stationary process, number of snapshots is quite limited.

The short-term stationarity assumption is often true for the problem of passive source localization, and there are some practical concerns regarding the number of snapshots. ${ }^{2}$ In this section, we want to apply some Bayesian performance bounds for two fundamental issues. First, given the SNR, how many snapshots are required to achieve the asymptotic performance. Second, how to predict the performance in the snapshot-deficient case.

We have already seen that the snapshot number $(L)$ is explicitly included in performance bounds through (3.33), (3.35) and (3.45). We evaluate the Bayesian CRB and ZZB for the above source range estimation problem. The number of snapshots varies from 1 to 48 , and the SNR is fixed at $-3 \mathrm{~dB}$. The results are shown in Fig. 6-4, and the MLE simulations are also plotted. A similar threshold behavior is observed in regard to the number of snapshots. While the CRB is almost a linear function of the snapshot number (in logarithmic scale), the ZZB does follow the MLE simulations. To operate in the asymptotic region, at this SNR, we should use 14 or more

\footnotetext{
${ }^{2}$ For example, Ref. [7] has discussed the snapshot deficiency due to the stationarity duration limits imposed by source transiting the resolution cell, and the bandwidth limits to avoid the phase distortion in estimation of the covariance matrix. As mentioned there, most adaptive algorithms rely on estimation of a sample covariance matrix; without enough snapshots, this matrix is rank-deficient or poorly conditioned.
} 


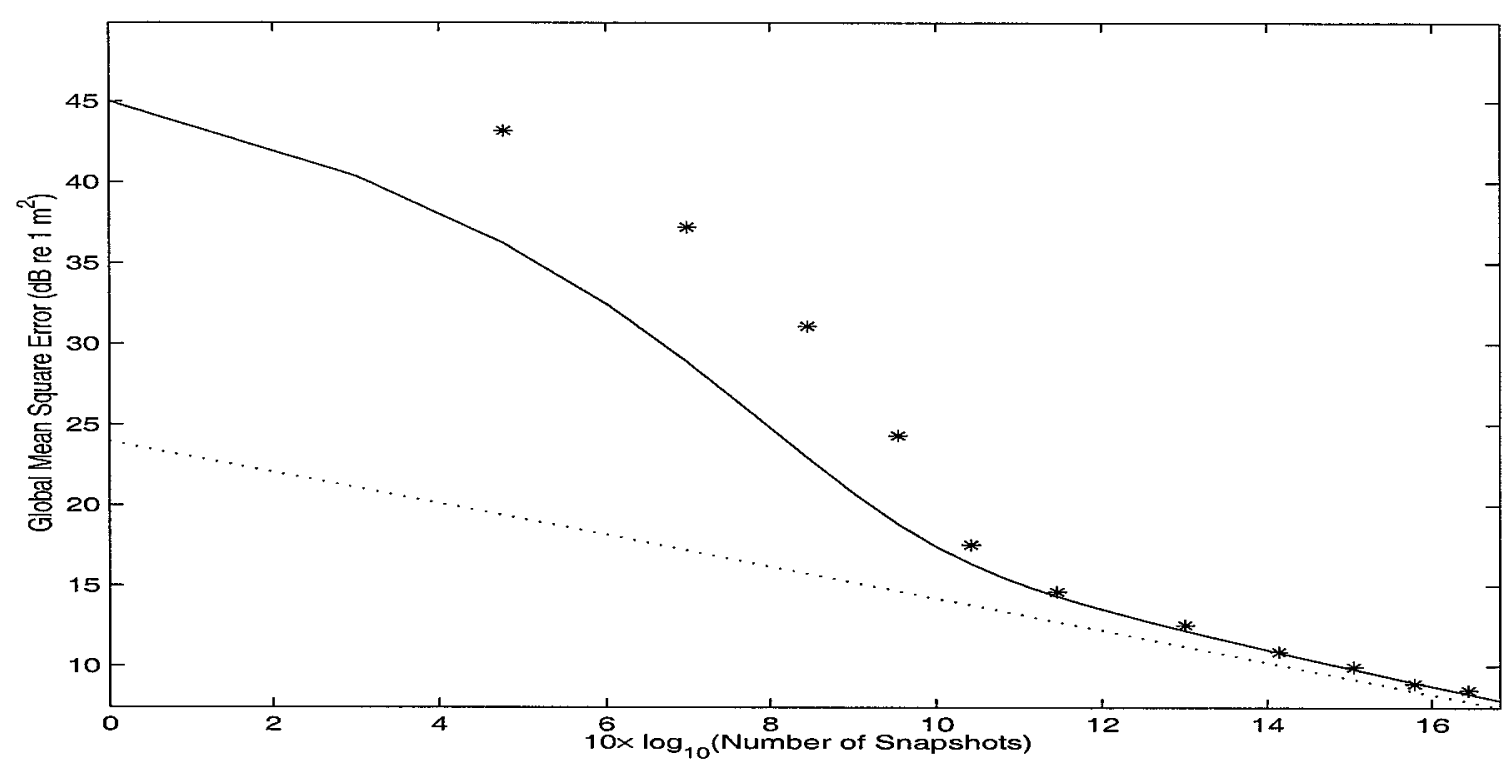

Figure 6-4: Performance bound as a function of the snapshot number for source range estimation. Solid line: ZZB; dotted line: BCRB; and *: MLE simulations. Sensor-averaged SNR is $-3 \mathrm{~dB}$.

independent snapshots. Considering the sensor number is 16 in this example, 16 or more snapshots would be preferred.

Fig. 6-5 shows the two-point estimation errors at different ambiguity points. We can clearly see the sidelobe contributions when the snapshot number varies from 5 to 25 , and the sidelobe errors dominate when the number of snapshots is smaller than 10.

In Fig. 6-5(b), we also notice that at $\mathrm{SNR}=-3 \mathrm{~dB}$, even with 25 snapshots the distant mainlobe point contributes more to the estimation error than the close mainlobe point. Accordingly, the CRB is not closely achieved. The opposite is also true: with a few snapshots, the CRB can not be closely achieved even at very high SNR. Let us look at an extreme case with only one snapshot available, as given in Fig. 6-6. Among three Bayesian bounds, only the ZZB is within $5 \mathrm{~dB}$ from the MLE simulations for SNR up to $20 \mathrm{~dB}$. This is again explained by noting that the sidelobe contribution is always high (Fig. 6-7). We would expect some intersections in error curves associated with the sidelobe points and the mainlobe points, but at a practically impossible high SNR. This is understandable because a few (even three 


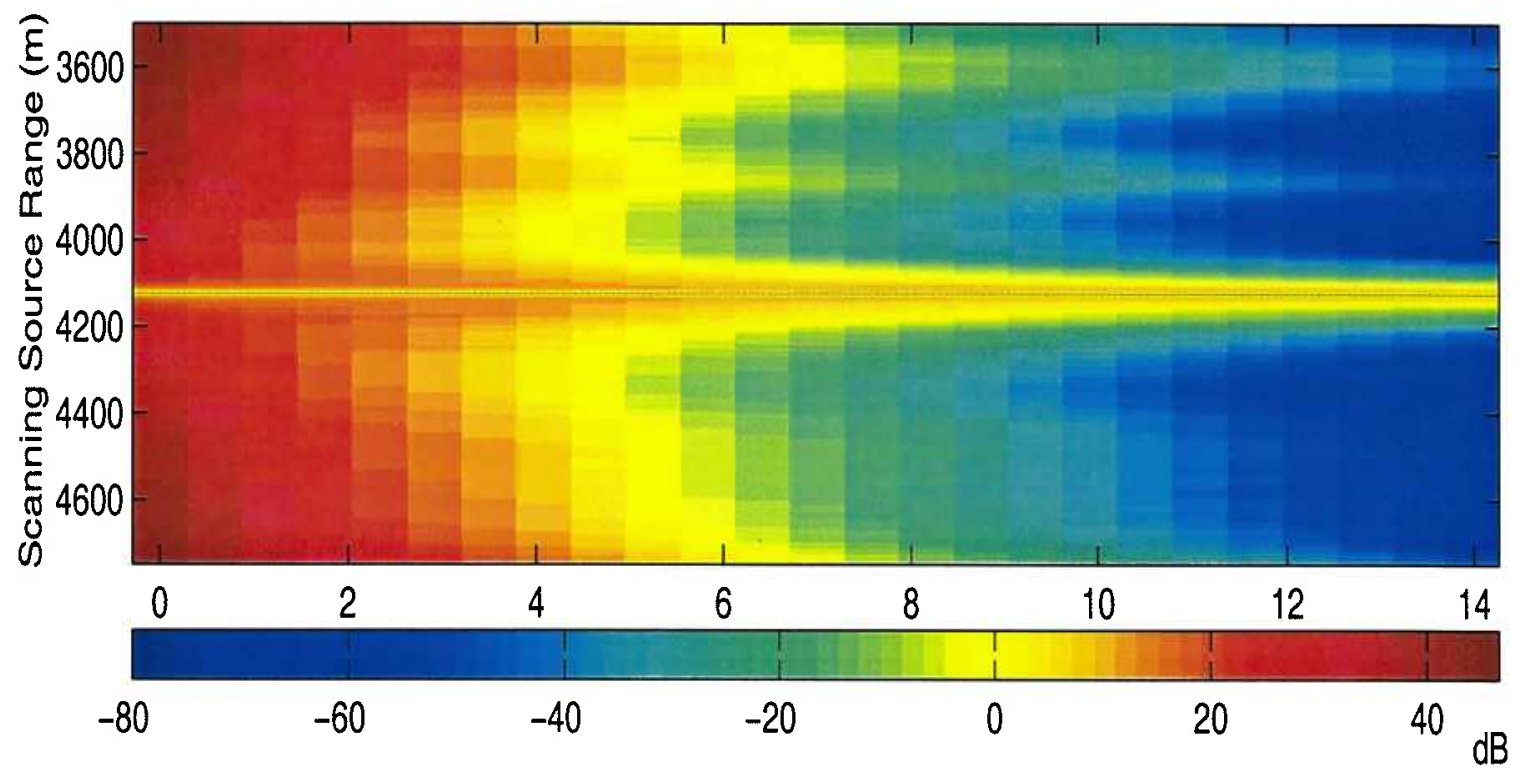

(a)

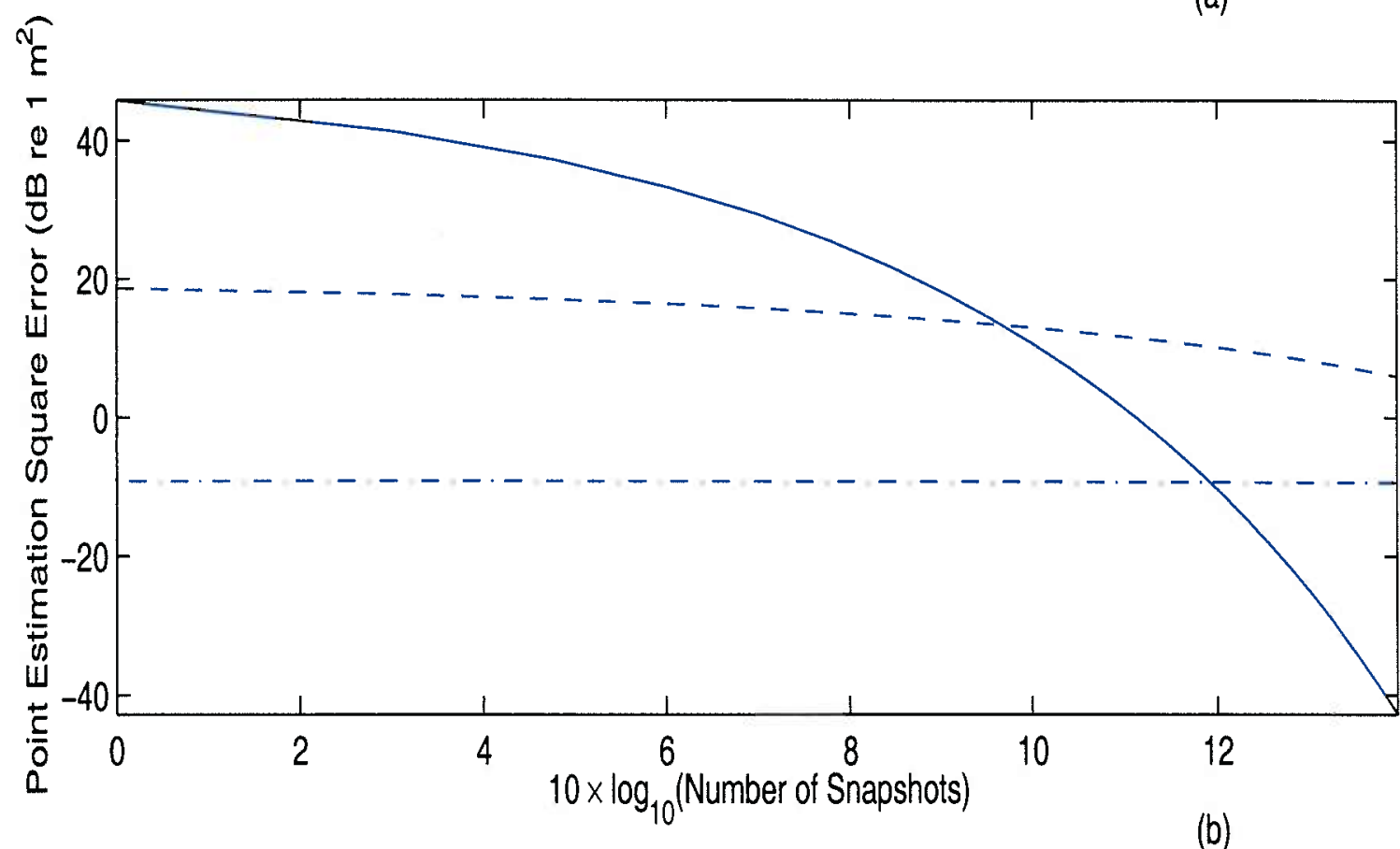

Figure 6-5: Probabilistic square errors as a function of the snapshot number: (a) at each scanning source range (The horizontal line in the middle corresponds to the true source range); (b) at representative ambiguity points - close mainlobe point (4124.5 $\mathrm{m}$, dashdot line), distant mainlobe point ( $4110.5 \mathrm{~m}$, dashed line), and distant sidelobe point (3580.5 m, solid line). 


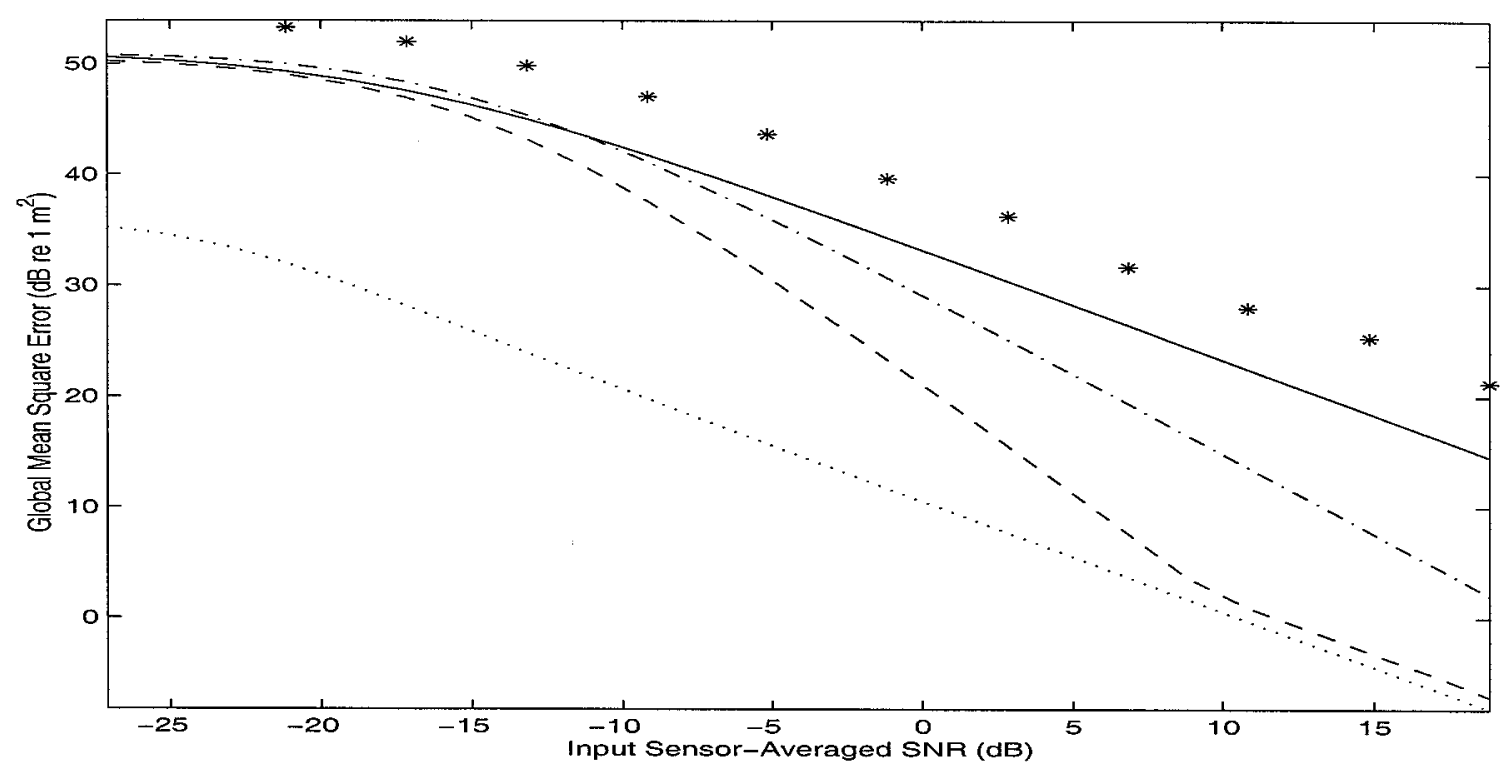

Figure 6-6: Performance bound evaluation for source range estimation with a single snapshot. Solid line: ZZB; dashdot line: multiple-test point WWB; dashed line: one-test point WWB; dotted line: BCRB; and *: MLE simulations.

or four) snapshots would be needed to tell the statistics of a random process.

Therefore, in terms of the effect on performance, the number of snapshots and the signal-to-noise ratio are not necessarily coupled according to an inverse-linear relation. In any case, the Ziv-Zakai bound gives the closest performance prediction.

\subsubsection{Incoherent Frequency Averaging}

Broadband processing has been investigated in the application of matched-field methods, aiming at exploiting the source frequency structure. For a multi-tone, random stationary source, a typical approach is incoherently averaging the ambiguity outputs across frequencies $[9,17,51]$. Because the sidelobe positions are frequency-dependent, in this way one would expect to average out the sidelobes and thus improve the peakto-sidelobe ratio. A rigorous analysis of incoherent frequency averaging is difficult because first, the frequency-dependence of the sidelobe position is complicated (cf. Section 4.1.1), and second, how the sidelobe structure (level and position) determines the estimation performance needs to be quantitatively analyzed. In this section, we would look at the second issue using the Bayesian performance bounds and error 


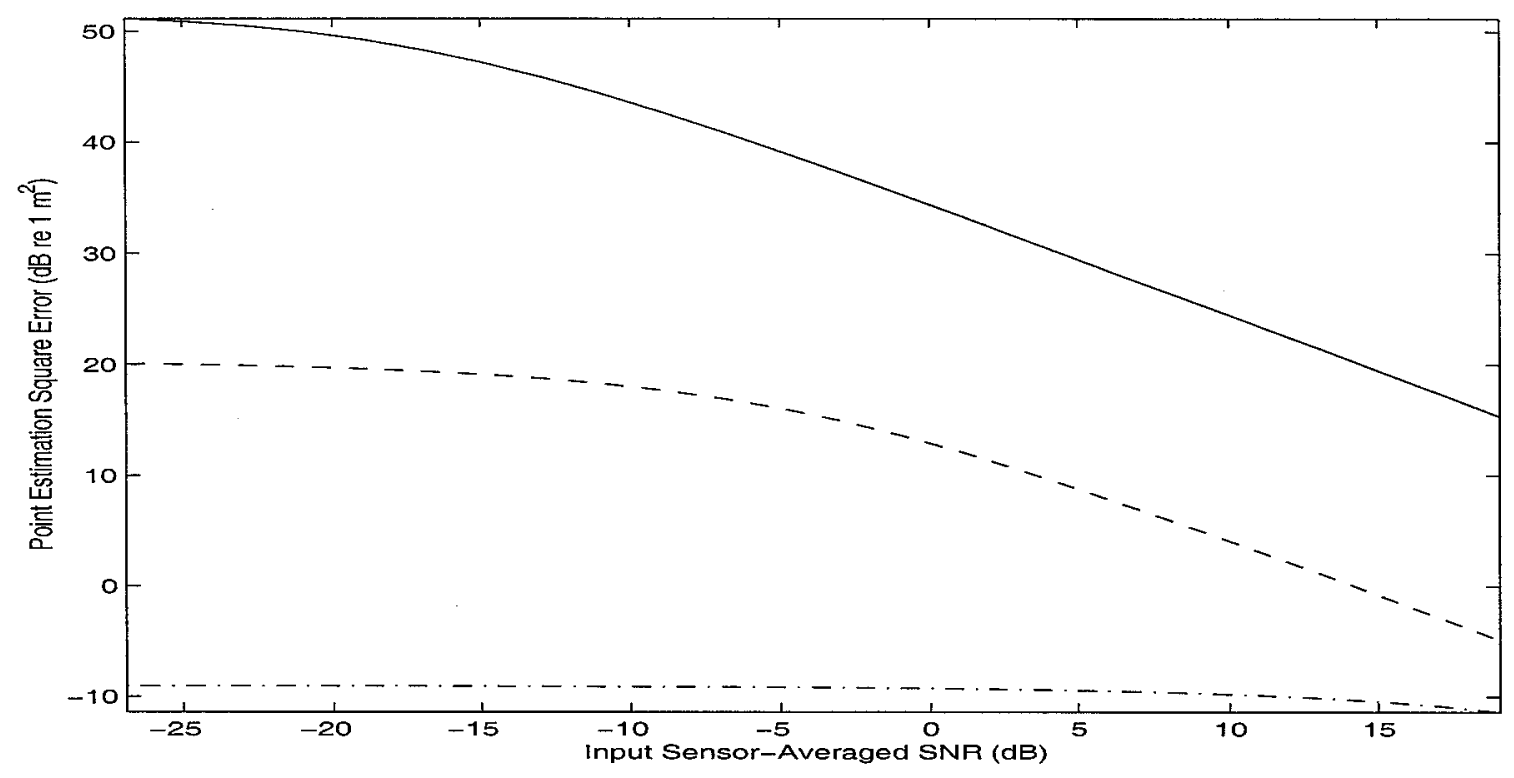

Figure 6-7: Probabilistic square errors as a function of SNR at different ambiguity points: close mainlobe point (4124.5 m, dashdot line); distant mainlobe point (4110.5 $\mathrm{m}$, dashed line); and distant sidelobe point ( $3580.5 \mathrm{~m}$, solid line). Only one snapshot is used.

analysis we have developed.

Back to the source range estimation problem, we now use ten pilot tones uniformly spread from $53 \mathrm{~Hz}$ to $197 \mathrm{~Hz}$. To incorporate more ambiguity points, we assume the source range varies from $3000 \mathrm{~m}$ to $6000 \mathrm{~m}$. For each pilot tone, we assume ten snapshots available. As derived in Appendix B, the maximum likelihood estimate is given by

$$
\hat{r}_{M L}=\arg \max _{r} \sum_{m=1}^{M} \sum_{l=1}^{L}\left|\mathbf{R}_{l}\left(f_{m}\right)^{\dagger} \mathbf{g}\left(f_{m}, r\right)\right|^{2} .
$$

This is a linear summation across frequencies.

Fig. 6-8 presents the MLE simulation results as well as the performance bounds. For the multi-frequency ZZB, the error approximation developed in Appendix C.2.2 is used. This error approximation works well as seen from the ZZB (black line). Compared to the single-frequency case (Fig. 6-1), the multi-frequency bounds (as well as the MLE simulations) are nearly shifted versions toward low SNR. The threshold SNR predicted by the ZZB is now about $-12 \mathrm{~dB}$. 


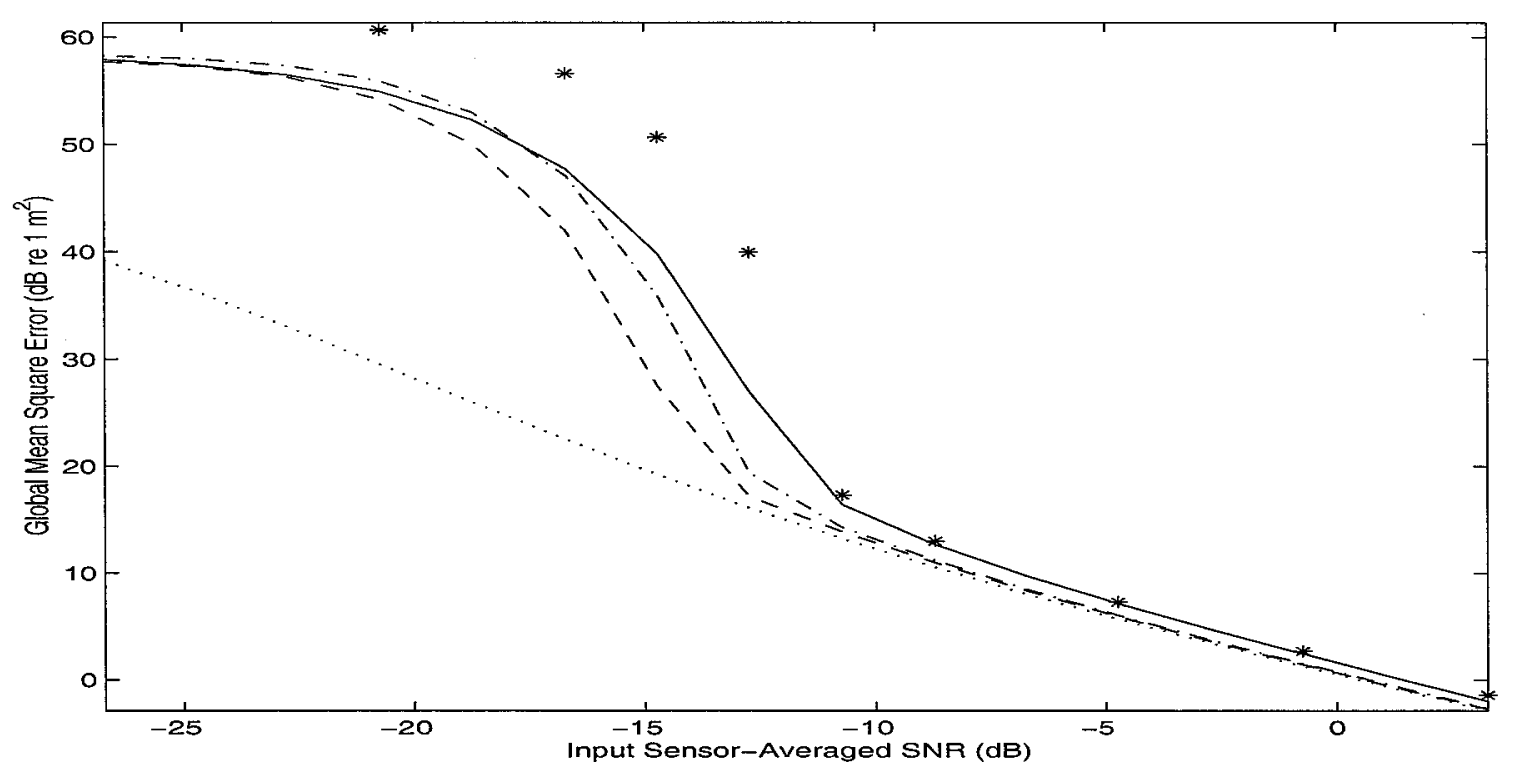

Figure 6-8: Performance bound evaluation for source range estimation with ten frequency components and ten snapshots for each frequency. Solid line: ZZB; dashdot line: multiple-test point WWB; dashed line: one-test point WWB; dotted line: $\mathrm{BCRB}$; and *: MLE simulations.

The above results do not justify the underlying reasoning for incoherent frequency averaging. Note that we now have 10 (frequencies) $\times 10$ (snapshots/frequency) independent measurements, ten times of that for the single-frequency case. This contributes to about 5 to $10 \mathrm{~dB}$ SNR gain. To look at the sidelobe effect after incoherent frequency averaging, we now use a single snapshot for each frequency so the number of independent measurements is the same as the single-frequency case. The results (Fig. 6-9) are similar to those for single-frequency case (Fig. 6-1). To better view the difference, we plot the Ziv-Zakai bound evaluated for single-frequency component at $53 \mathrm{~Hz}, 101 \mathrm{~Hz}$ and $197 \mathrm{~Hz}$, individually, as well as ten frequency components by incoherent averaging. As shown in Fig. 6-10, the bound at $101 \mathrm{~Hz}$ is a little higher in the asymptotic region, while the $53 \mathrm{~Hz}$ one enters the transition region a bit earlier. However, the ten-frequency one is hardly distinguishable from that at the highest frequency, only a bit smaller in the transition region.

This doesn't mean the incoherent frequency averaging cannot reduce the sidelobe effect. Fig. 6-11 shows some ambiguity functions given the true source range at $4500 \mathrm{~m}$. As expected, the signal field is more ambiguous at low frequency and 


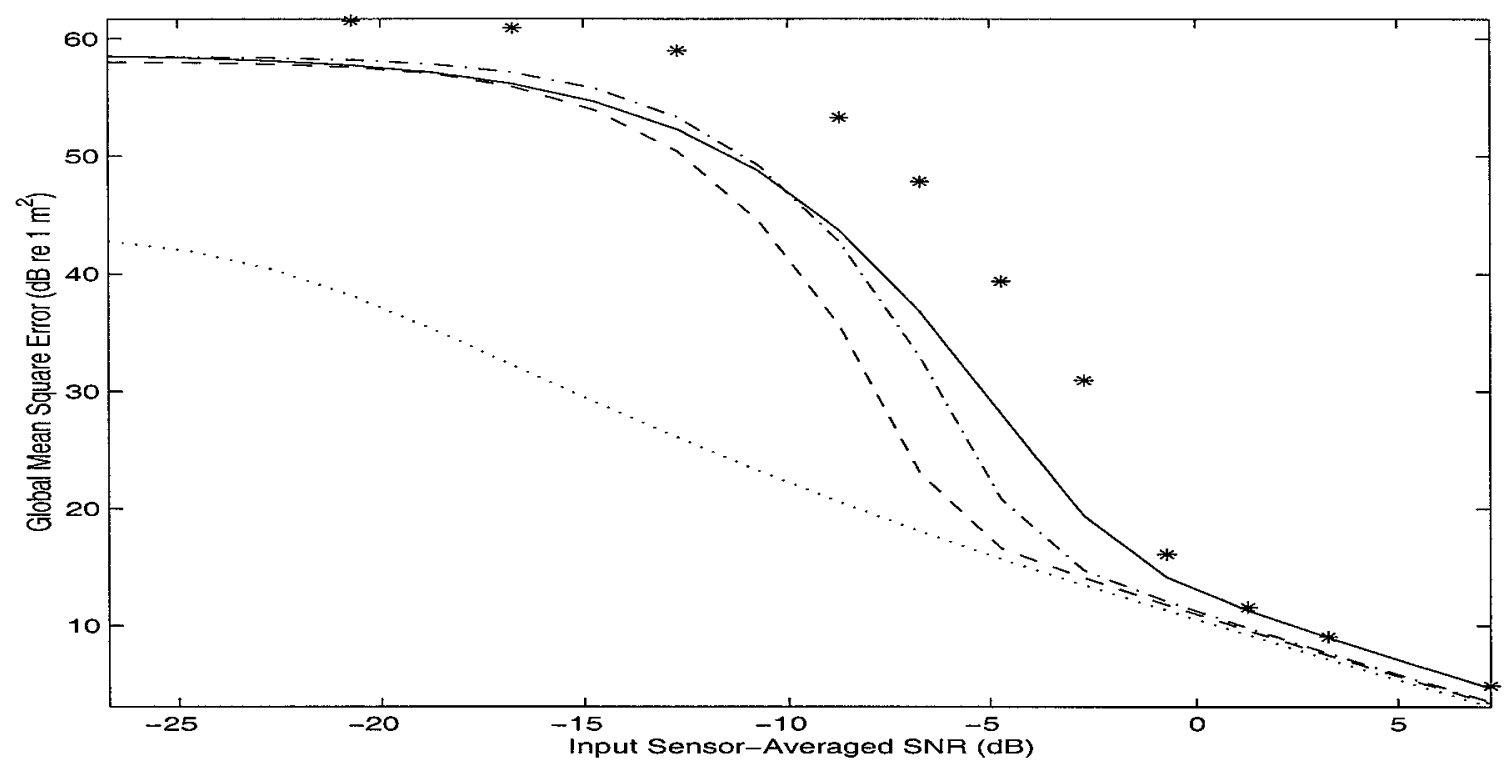

Figure 6-9: Performance bound evaluation for source range estimation with ten frequency components and single snapshot for each frequency. Solid line: ZZB; dashdot line: multiple-test point WWB; dashed line: one-test point WWB; dotted line: BCRB; and *: MLE simulations.

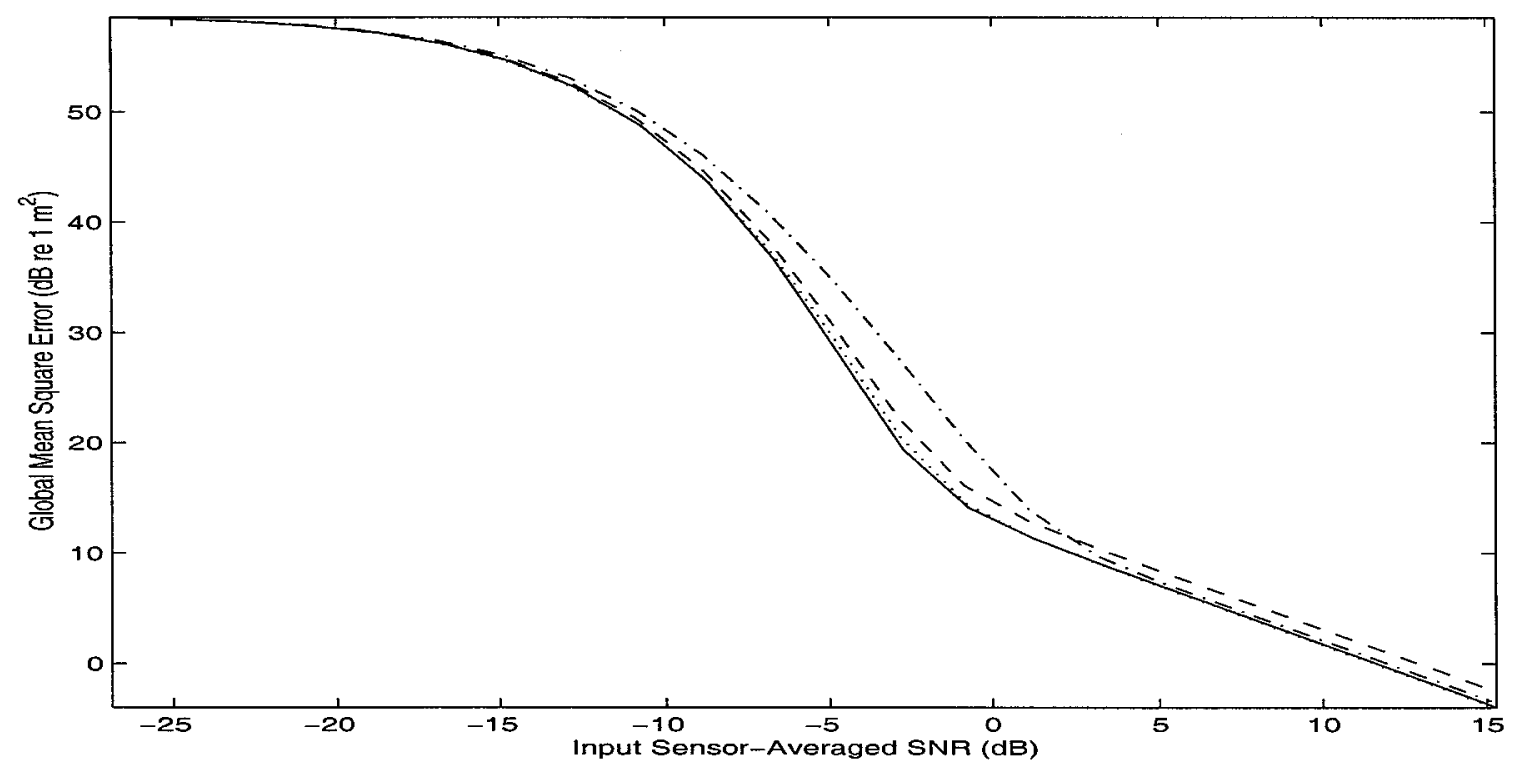

Figure 6-10: Ziv-Zakai bound for source range estimation with single frequency component and ten snapshots: $53 \mathrm{~Hz}$ (Dashdot line), $101 \mathrm{~Hz}$ (Dashed line), and $197 \mathrm{~Hz}$ (Dotted line); with ten frequency components and single snapshot for each frequency (Solid line). 


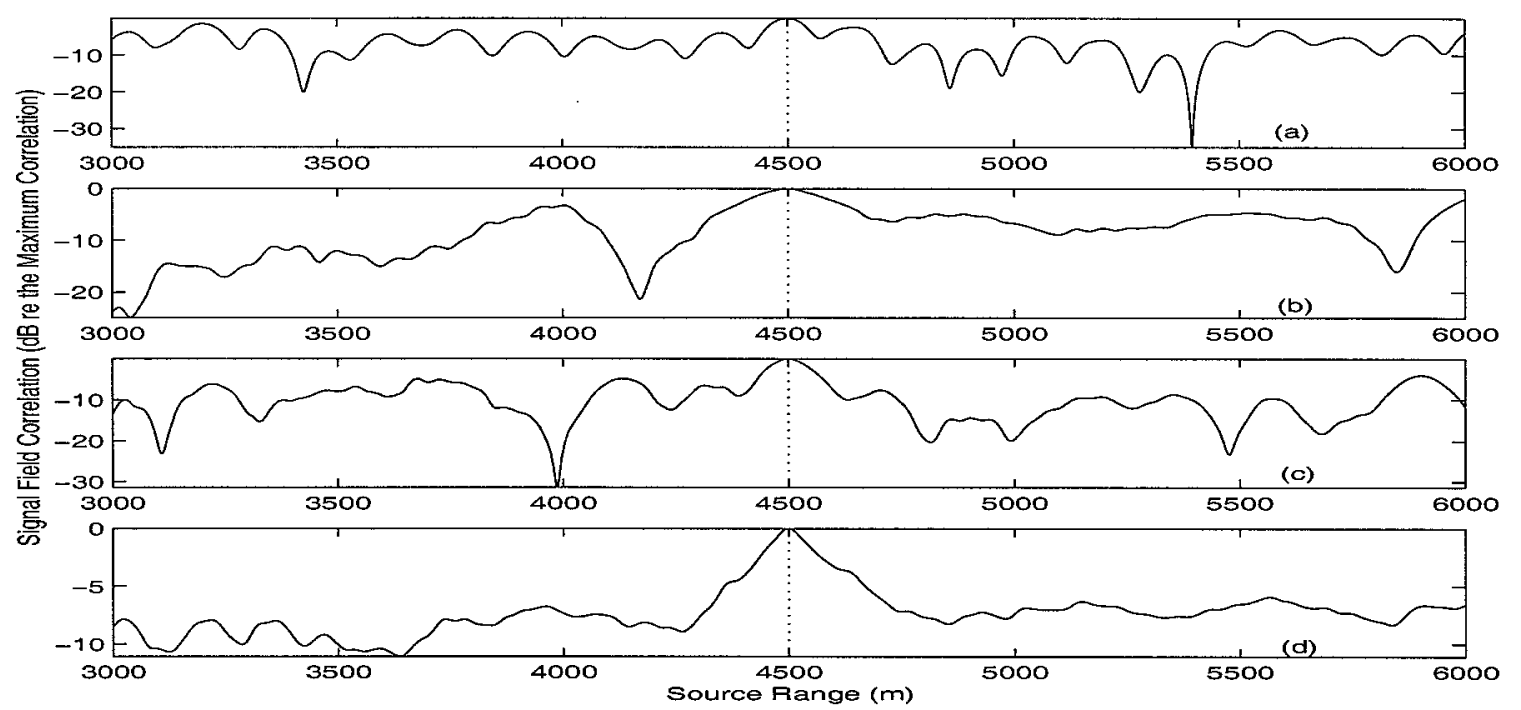

Figure 6-11: Example signal ambiguity functions for source range estimation at different frequencies: (a) $53 \mathrm{~Hz}$; (b) $101 \mathrm{~Hz}$; (c) $197 \mathrm{~Hz}$; and (d) incoherently averaged over ten frequencies. The dotted line indicates the true source range.

less ambiguous at high frequency. The frequency-averaged ambiguity function does have level-reduced sidelobes. This can also be observed from the point estimation errors associated with different representative ambiguity points (Fig. 6-12). With single-frequency component $(53 \mathrm{~Hz}, 101 \mathrm{~Hz}$ and $197 \mathrm{~Hz})$, the estimation error by a representative sidelobe point is larger than that by a representative mainlobe point at $\mathrm{SNR} \approx 3 \mathrm{~dB}, 1 \mathrm{~dB}$, and $-1 \mathrm{~dB}$, individually. These are about consistent with the bound's evaluations. However, with ten frequency components, the sidelobe error is larger than the mainlobe error at $\mathrm{SNR} \approx-2 \mathrm{~dB}$. This is attributed to reduced sidelobe levels.

On the other hand, at $\mathrm{SNR}=-1 \mathrm{~dB}$, although error at any single sidelobe point is smaller than that at the representative mainlobe point, there are many points making comparable contributions (Fig. 6-13). This is consistent with the frequency-averaged ambiguity function, which, compared to the single-frequency ones, maintains a higherlevel baseline across the parameter interval. As a result, the overall mean square error is close to that of $197 \mathrm{~Hz}$ and demonstrates a threshold behavior at this SNR.

To conclude this section, the incoherent frequency averaging does reduce the side- 

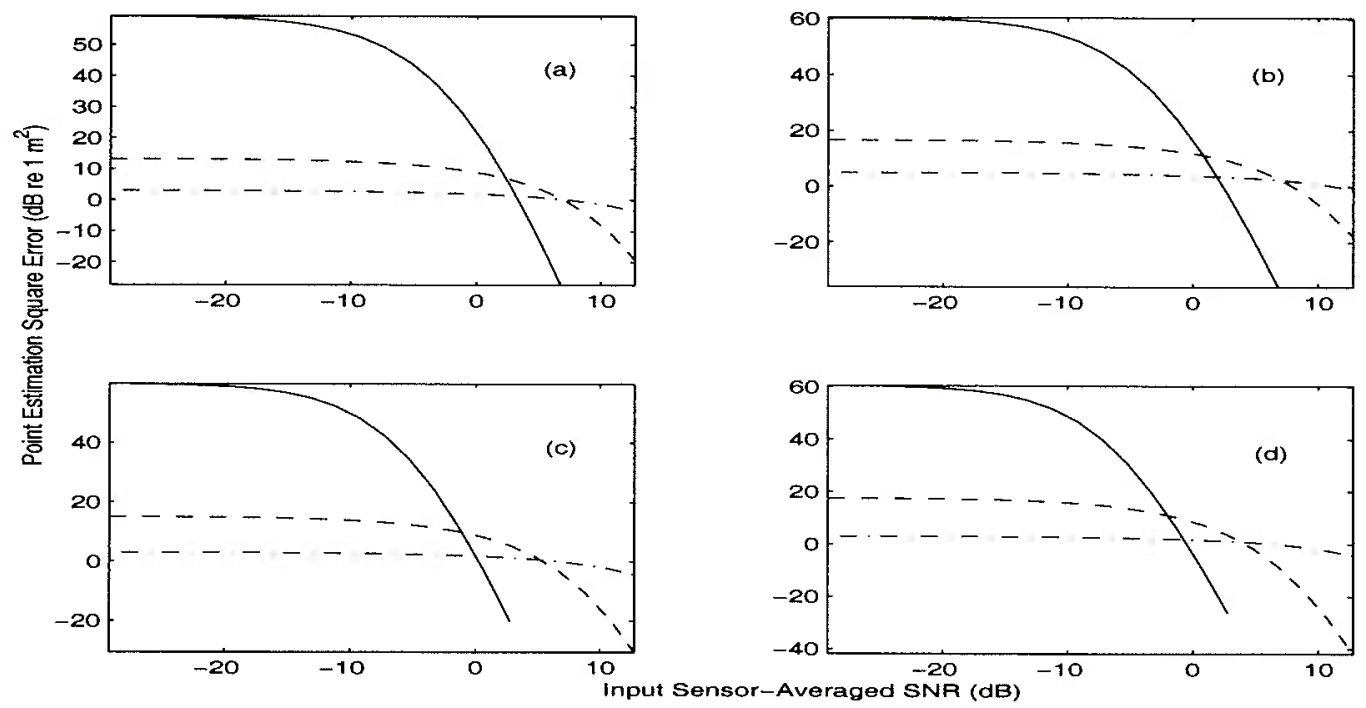

Figure 6-12: Probabilistic square errors as a function of SNR at a close mainlobe point (dashdot line), a distant mainlobe point (dashed line), and a distant sidelobe point (solid line): (a) $53 \mathrm{~Hz}$; (b) $101 \mathrm{~Hz}$; (c) $197 \mathrm{~Hz}$; and (d) incoherently averaged over ten frequencies.

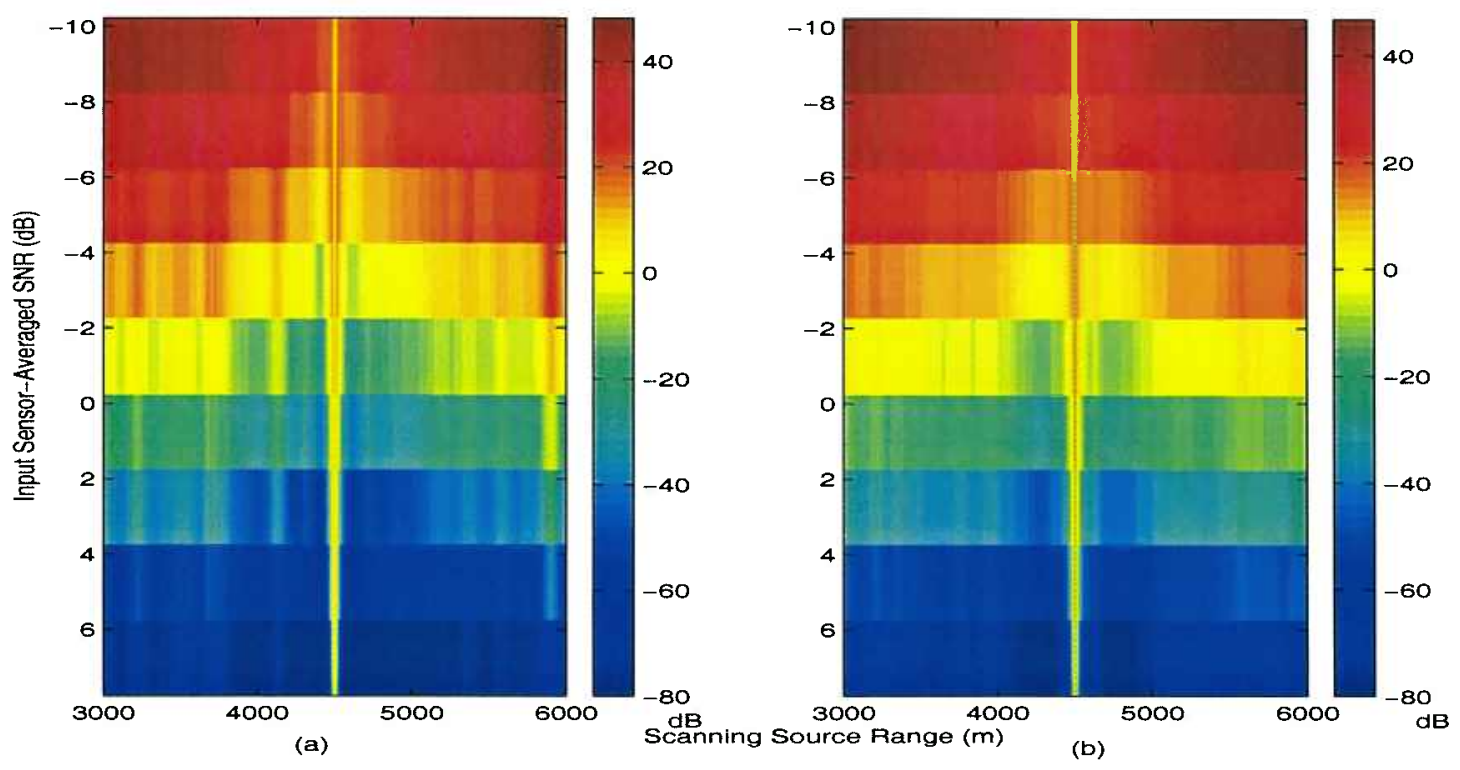

Figure 6-13: Probabilistic square error as a function of SNR at each scanning source range: (a) $197 \mathrm{~Hz}$; (b) incoherently averaged over ten frequencies. The vertical line in the middle corresponds to the true source range. 
lobe level but increase the overall ambiguity background as well. In this example, the resulted performance is similar to that achieved by a high frequency component. Even in this case, the frequency-averaging may still be preferred because the ambiguity peak at high frequency is often more sensitive to environmental perturbations [51]. To improve the overall performance, one needs to judiciously choose a set of frequency components. In addition, for such an averaged ambiguity function, the threshold SNR predicted by the two-point estimation error analysis can be a bit lower.

\subsection{Environmental Parameter Estimation}

Before we go to more complicated source localization problems, such as those including environmental uncertainty/mismatch, let us look at several examples of environmental parameter estimation.

As mentioned in Section 4.1.3, for most bottom properties (e.g., wave-speed, attenuation and density), the associated signal field is much less ambiguous, compared to the source location estimation case. This is because a bottom parameter enters the signal field through some high-order modes, which are often less significant at long-range. Accordingly, some ambiguity points on a wide mainlobe are the major factors in determining the estimation performance, and we can hardly see any threshold behavior due to sidelobes. For example, in estimation of sediment wave-speeds (top and bottom) under the example SWellEX-3 environment, the asymptotic region (linear SNR-dependence) almost extends to the no-information region at very low SNR (Figs. 6-14 and 6-15). At input SNR =-2 dB (output SNR $\approx 15 \mathrm{~dB}$ ), the resolution is about $6 \mathrm{~m} / \mathrm{s}$ for both top and bottom wave-speeds.

In fact, to estimate those ambiguity-free parameters, a local algorithm often works well, which iteratively improves a starting environmental model through a gradientdirected searching scheme [57]. For a multiple-parameter estimation problem, the ambiguity surface often shows a multi-modal structure, and a local algorithm can be trapped on a local optimum model, particularly when some geometry parameters are included. As discussed in Section 4.1.3, for the given SWellEX-3 environment, the 


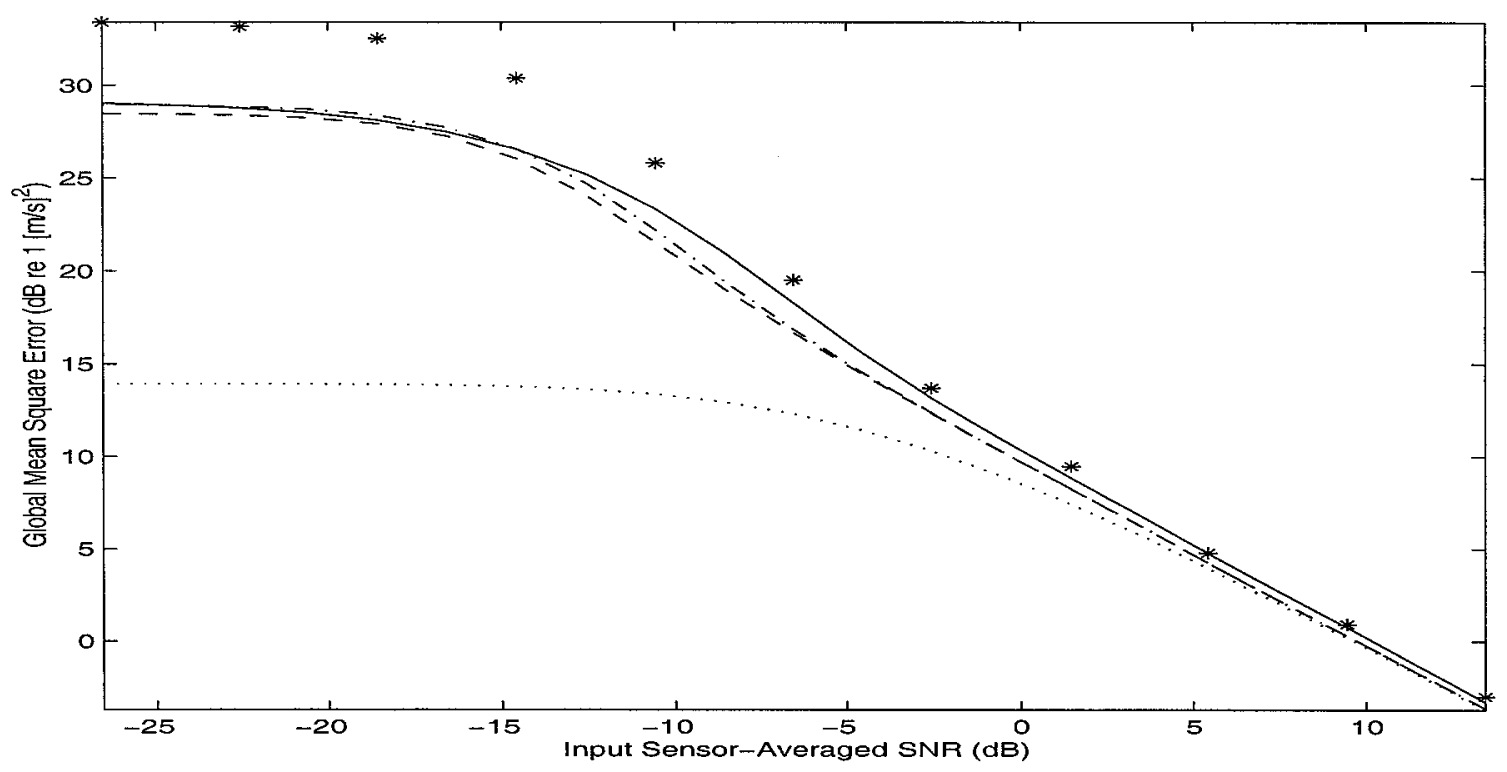

Figure 6-14: Performance bound evaluation for sediment top wave-speed estimation. Solid line: ZZB; dashdot line: multiple-test point WWB; dashed line: one-test point WWB; dotted line: BCRB; and *: MLE simulations.

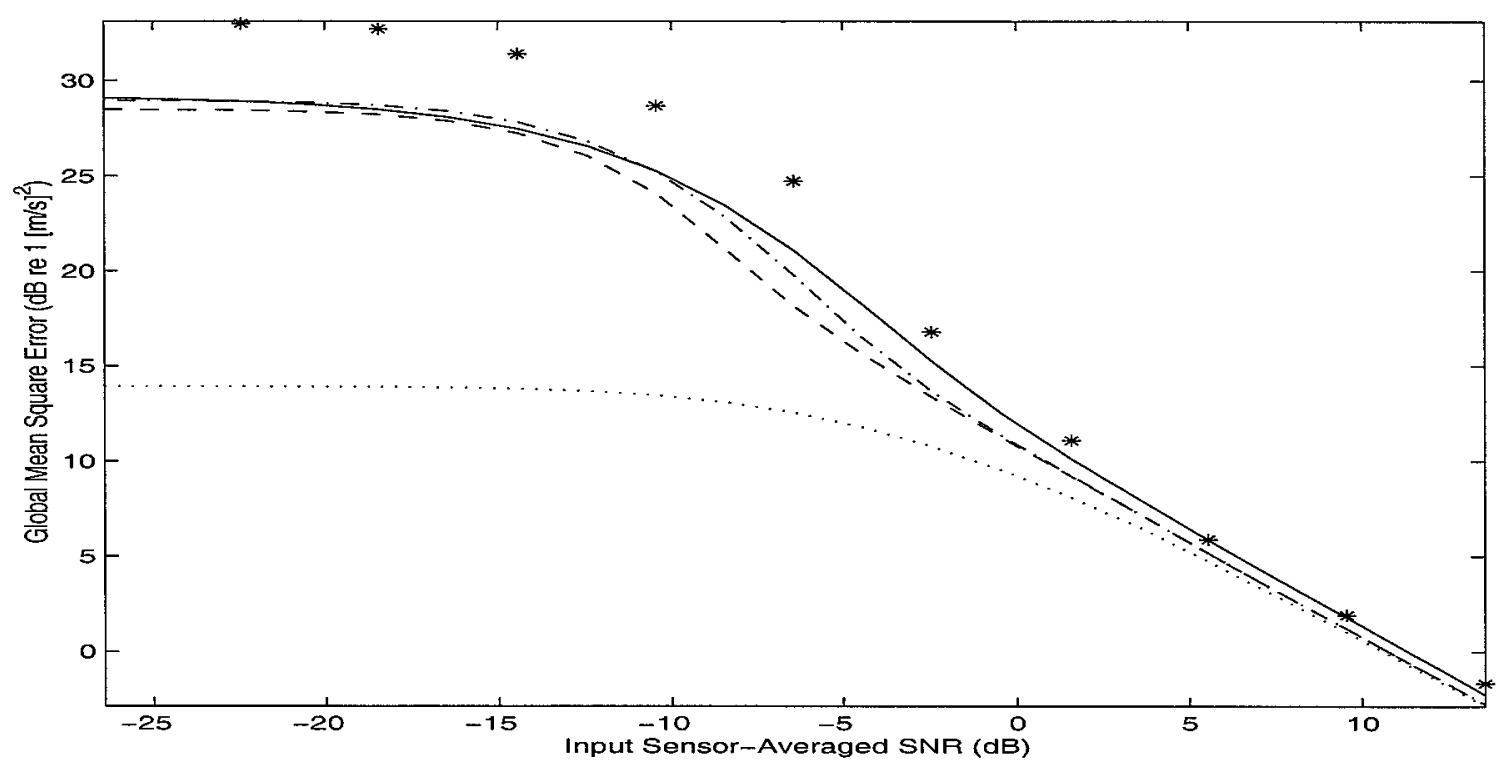

Figure 6-15: Performance bound evaluation for sediment bottom wave-speed estimation. Solid line: ZZB; dashdot line: multiple-test point WWB; dashed line: one-test point WWB; dotted line: BCRB; and *: MLE simulations. 
sediment layer plays a significant role in determining the signal field. Thus even for a single geometry parameter (sediment thickness), the associated signal field demonstrates a strong ambiguity (cf. Fig. 4-6).

We evaluate three Bayesian bounds for this sediment thickness estimation problem and the results are given in Fig. 6-16. Also plotted is the MLE performance simulation. The chosen parameter sampling step is $0.01 \mathrm{~m}$ to maintain a good field linearity. Some interesting behaviors are observed. First, the Bayesian CRB is about $5 \mathrm{~dB}$ below the MLE mean square error even in the high SNR region. This is because the local Fisher information shows a strong inhomogeneity across the parameter interval. Second, the one-test point WWB doesn't converge to the CRB. As seen in Fig. 6-17, the estimation error due to the second point from the true parameter point is always higher than that due to the first point, and thus the optimum test point of the WWB doesn't go to zero. Besides, the WWB uses a different approach to combine the local performance at each individual parameter point. Third, the multiple-test point WWB doesn't converge to the one-test point one. This says even at high SNR the estimation error is not determined by a single close mainlobe point.

In any case, the ZZB gives consistent tight performance predictions. The predicted threshold SNR is about $8 \mathrm{~dB}$. The threshold SNR is also estimated using the approach described in Section 4.5.1 and the results are given in Table 6.2. Each prediction at a given true parameter point is obtained as described in Fig. 6-17. The mean point of 19 such predictions is $8 \mathrm{~dB}$, and the 68-percentile point is $10 \mathrm{~dB}$, which is a good indication of the threshold in MLE simulations (Fig. 6-16).

Compared to the range estimation case, both the mainlobe and sidelobe behaviors in thickness estimation are highly inhomogeneous: less ambiguous at shallower depths; more ambiguous at deeper depths. Indeed, at deeper depths, the nearby correlation levels are pretty high, and some small sidelobe peaks overlie the high-level mainlobe. This could be attributed to the attenuation factor, which makes the rather ambiguous field smoother as the sediment depth increases. 


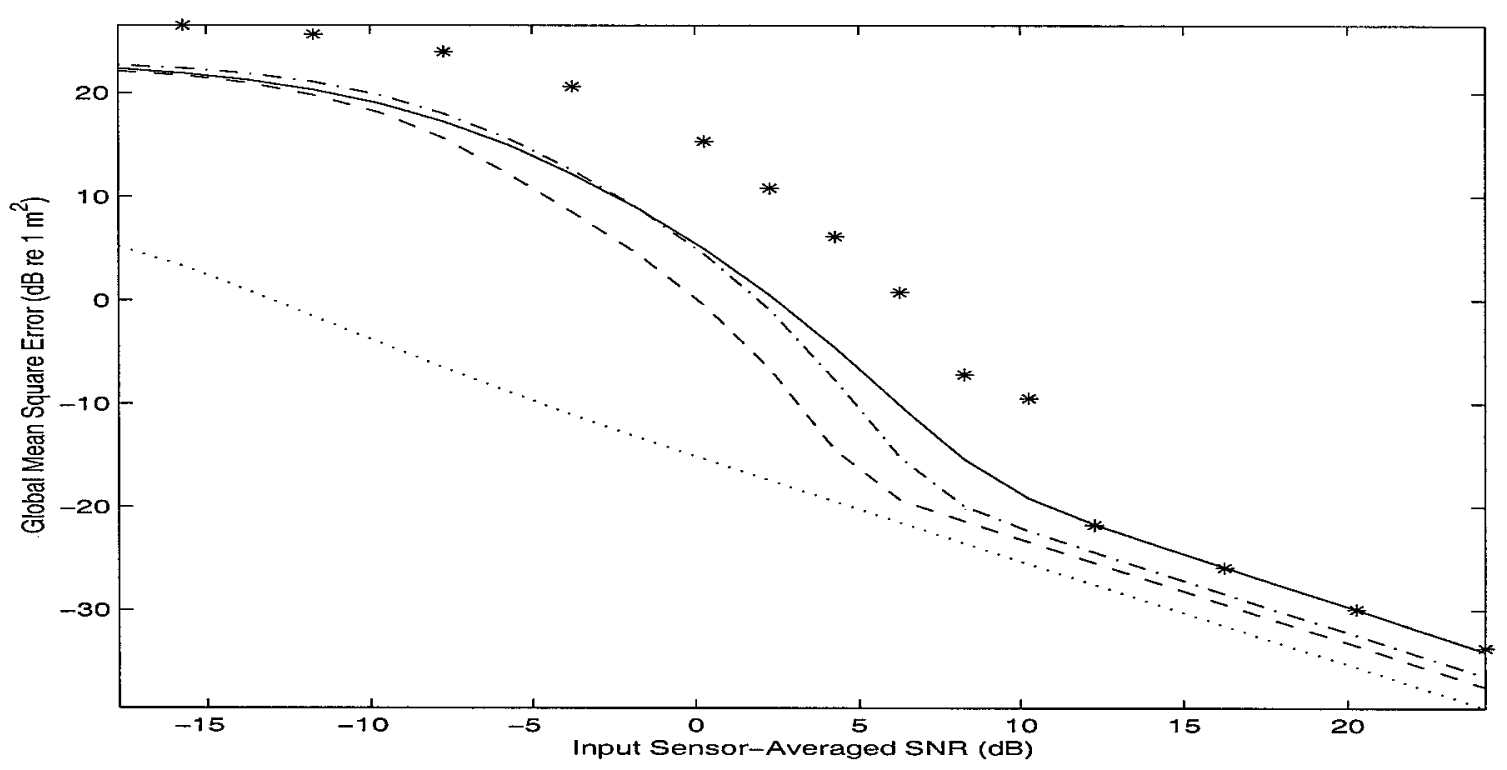

Figure 6-16: Performance bound evaluation for sediment thickness estimation with uniform prior distribution. Solid line: ZZB; dashdot line: multiple-test point WWB; dashed line: one-test point WWB; dotted line: BCRB; and *: MLE simulations.

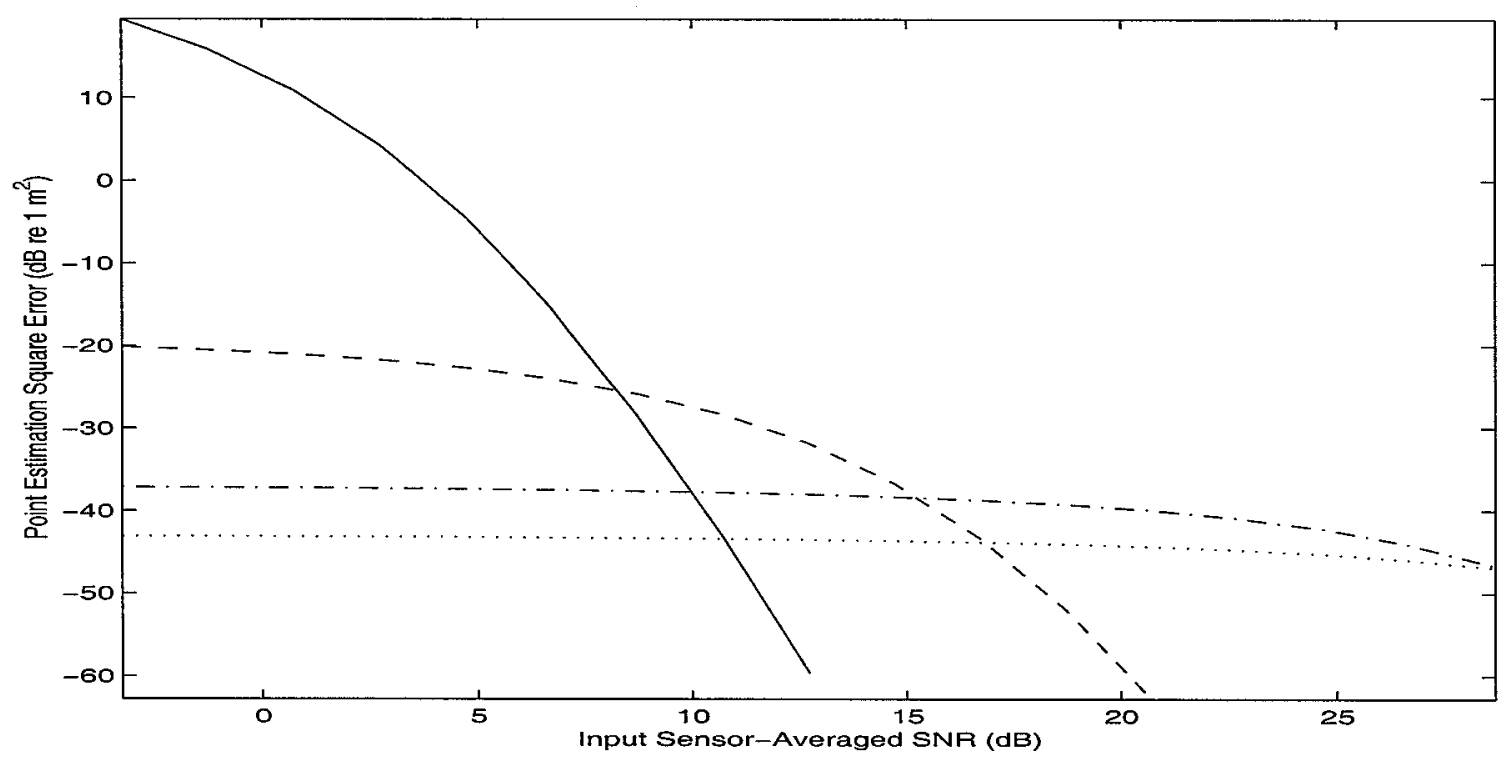

Figure 6-17: Probabilistic square errors for sediment thickness estimation as a function of SNR at different ambiguity points: close mainlobe point $(24.49 \mathrm{~m}$, dotted line); close mainlobe point ( $24.48 \mathrm{~m}$, dashdot line); distant mainlobe point ( $24.34 \mathrm{~m}$, dashed line); and distant sidelobe point (60.32 m, solid line). The true thickness is at $24.50 \mathrm{~m}$. 
Table 6.2: Threshold SNR predictions at individual true parameter points in sediment thickness estimation.

\begin{tabular}{|c|r||c|r|}
\hline Sediment Thickness $(\mathrm{m})$ & $\mathrm{SNR}_{1}(\mathrm{~dB})$ & Sediment Thickness $(\mathrm{m})$ & $\mathrm{SNR}_{1}(\mathrm{~dB})$ \\
\hline \hline 14.5 & 5 & 39.5 & 8 \\
\hline 17.0 & 4 & 42.0 & 10 \\
\hline 19.5 & 6 & 44.5 & 11 \\
\hline 22.0 & 6 & 47.0 & 12 \\
\hline 24.5 & 8 & 49.5 & 9 \\
\hline 27.0 & 7 & 52.0 & 10 \\
\hline 29.5 & 6 & 54.5 & 10 \\
\hline 32.0 & 6 & 57.0 & 10 \\
\hline 34.5 & 7 & 59.5 & 9 \\
\hline 37.0 & 8 & & 10 \\
\hline \hline Mean Point & 8 & 68 -percentile Point & \\
\hline
\end{tabular}

\subsubsection{Gaussian Parameter Model}

The Gaussian distribution often makes more sense in describing the random model for an environmental parameter. This might be because it better describes one's confidence about that the parameter falls into gradually widened intervals. Given the mean $m_{\theta}$, and the standard deviation $\sigma_{\theta}$, the parameter distribution can be stated by

$$
p(\theta)=\frac{1}{\sqrt{2 \pi} \sigma_{\theta}} \exp \left(\frac{\left(\theta-m_{\theta}\right)^{2}}{2 \sigma_{\theta}^{2}}\right)
$$

Hence the Fisher information due to the a priori parameter distribution (cf. (3.12)) is now

$$
J_{A}=\frac{1}{\sigma_{\theta}^{2}}
$$

For general Bayesian estimation problem, the maximum a posteriori estimator (MAP) is shown to minimize the Bayes risk for a "hit-or-miss" cost function [43]. In previous examples, the usage of the maximum likelihood estimator is justified by 
assuming a uniform parameter distribution. Note that

$$
p(\theta \mid \mathbf{R})=\frac{p(\mathbf{R} \mid \theta) p(\theta)}{p(\mathbf{R})}
$$

When the prior parameter distribution does not favor one parameter over the others, the MLE is equivalent to the MAP. However, for a Gaussian prior distribution, we should use the true MAP algorithm, which for the given data model is stated by

$$
\hat{\theta}_{M A P}(\mathbf{R})=\arg \max _{\theta}\left[Q_{1}\left(f_{0}, \theta\right)+Q_{2}\left(f_{0}, \theta\right) \cdot \sum_{l=1}^{L}\left|\mathbf{R}_{l}^{\dagger}\left(f_{0}\right) \mathbf{g}\left(f_{0}, \theta\right)\right|^{2}+\ln p(\theta)\right]
$$

and $Q_{1}$ and $Q_{2}$ are defined in (B.6) and (B.7), respectively.

We now assume a Gaussian distribution for sediment thickness with $m_{\theta}=37 \mathrm{~m}$ and $\sigma_{\theta}=7.5 \mathrm{~m}$. For numerical evaluation, this distribution is cut off at $12 \mathrm{~m}$ and $62 \mathrm{~m}$. The results are shown in Fig. 6-18. Because the effective parameter interval is narrowed, the local performance variation is reduced such that the CRB, WWB and ZZB converge better at high SNR. The threshold SNR is about the same due to some significant close sidelobe points overlying the mainlobe. It is interesting to note that the MAP simulation has a peak at about $\mathrm{SNR}=-11 \mathrm{~dB}$. This is actually determined by the relative size of the a priori information term and the likelihood function term (see (6.13)). At high SNR, the likelihood function term dominates and the performance is similar to that of the MLE; as the SNR decreases, the a priori information term becomes important and drags the overall mean square error down to the variance of the a priori parameter distribution.

Although no example is presented for multi-dimensional environmental parameter estimation, discussions on some fundamental issues can be found in the sections followed, which consider multi-dimensional source and source/environmental parameter estimation. 


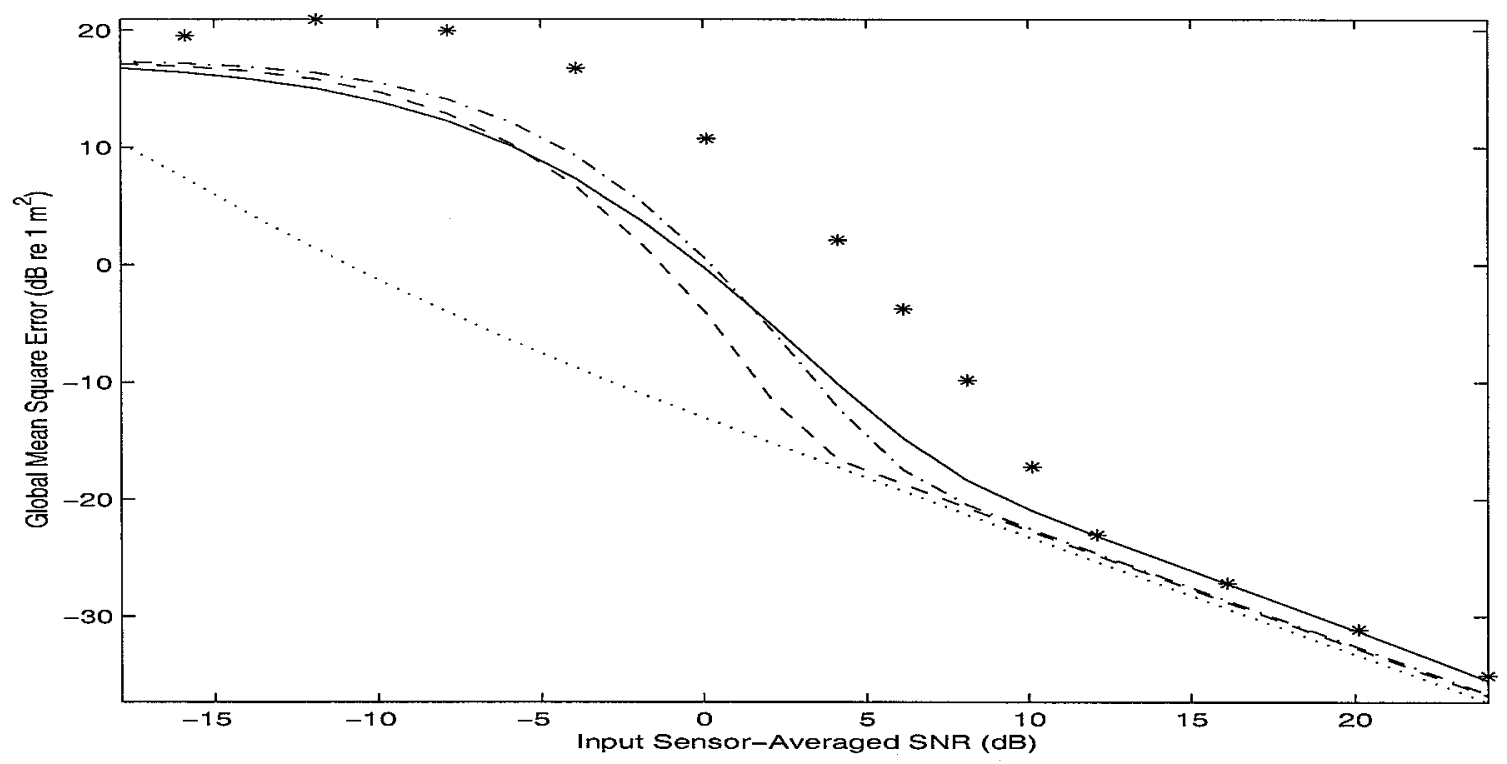

Figure 6-18: Performance bound evaluation for sediment thickness estimation with Gaussian prior distribution. Solid line: ZZB; dashdot line: multiple-test point WWB; dashed line: one-test point WWB; dotted line: BCRB; and *: MAP simulations.

\subsection{Source Range Estimation with Unknown Depth}

In previous examples, we have investigated the capabilities of three Bayesian performance bounds to capture the ambiguity in estimation of a single parameter. Generalization to estimation of two or more parameters is straightforward, and most of bound's properties still hold. The problem, however, is more complicated due to a number of factors.

First, the ambiguity function in multi-dimensional parameter space often shows strong multi-modal behavior. Given the true parameter position, one needs to find a set of important sidelobe points, which involves a global optimization problem. Second, there can be strong interactions (coupling) among parameters. This must be taken into account in understanding the resulted performance. Third, the signal field and the associated local property can be highly inhomogeneous across the parameter space. A Bayesian bound measures the averaged performance and thus can be misleading under strong local performance inhomogeneity. Finally, as a result, the computational effort can be significant.

Let us first consider a typical source localization problem, i.e., simultaneous 
range/depth estimation. Compared to the single range or depth estimation case, the associated signal field often shows a stronger ambiguity as well as inhomogeneity in a large scanning region of source location. Using the SWellEX-3 example, we assume an interval of [3500 4750] $\mathrm{m}$ for source range, [30 150] $\mathrm{m}$ for source depth. Again, we use ten snapshots of a single frequency component at $101 \mathrm{~Hz}$.

Fig. 6-19 displays the Ziv-Zakai bound as well as the MLE simulation results in range estimation. For comparison, the previous results for a fixed source depth are also plotted. The MLE simulation with unknown depth enters the transition region a bit earlier by $2 \mathrm{~dB}$. This is captured by the ZZB by observing the departure from the linear region, and the predicted resolution is about $10 \mathrm{~m}$ at input $\mathrm{SNR}=-3$ $\mathrm{dB}(1 \mathrm{~m}$ at input $\mathrm{SNR}=12 \mathrm{~dB})$. However, both cases surprisingly agree well in the asymptotic region.

Recall that in Section 4.3.3 we have discussed the effect on estimation of one parameter by introducing uncertainties in other parameters. This effect is incorporated using a projection of the multi-dimensional ambiguity function, which, in this example, is obtained by choosing the maximum of the range-depth ambiguity surface for each range point. Fig. 6-20 displays the eigenvectors of the high-SNR resolution matrix specified by the CRB; they are all almost parallel to either of the parameter axes, indicating a uniform weak coupling between range and depth. Thus, the ambiguity mainlobe shape is maintained with/without depth uncertainty (cf. Fig. 4-11). This explains the performance consistency in the asymptotic region (cf. Section 4.3.1).

The threshold behavior can then be analyzed based on the projected ambiguity function (see the dashdot line in Fig. 4-11). Given the true source range at $4125 \mathrm{~m}$, the resulted point estimation errors are plotted in Fig. 6-21. Compared to Fig. 4-18, the representative mainlobe points are about the same but the sidelobe point is now at $4724 \mathrm{~m}$ (cf. Fig. 4-11), and the threshold SNR is about $-2 \mathrm{~dB}$, one $\mathrm{dB}$ higher. Repeat this analysis for a preselected set of true parameter points, so we have a contour plot of the predicted threshold SNR within the entire parameter interval. As seen from Fig. 6-22, the signal field is inhomogeneous in regard to the sidelobe behavior: more ambiguous at mid-depth particularly for short- and long-ranges; and 


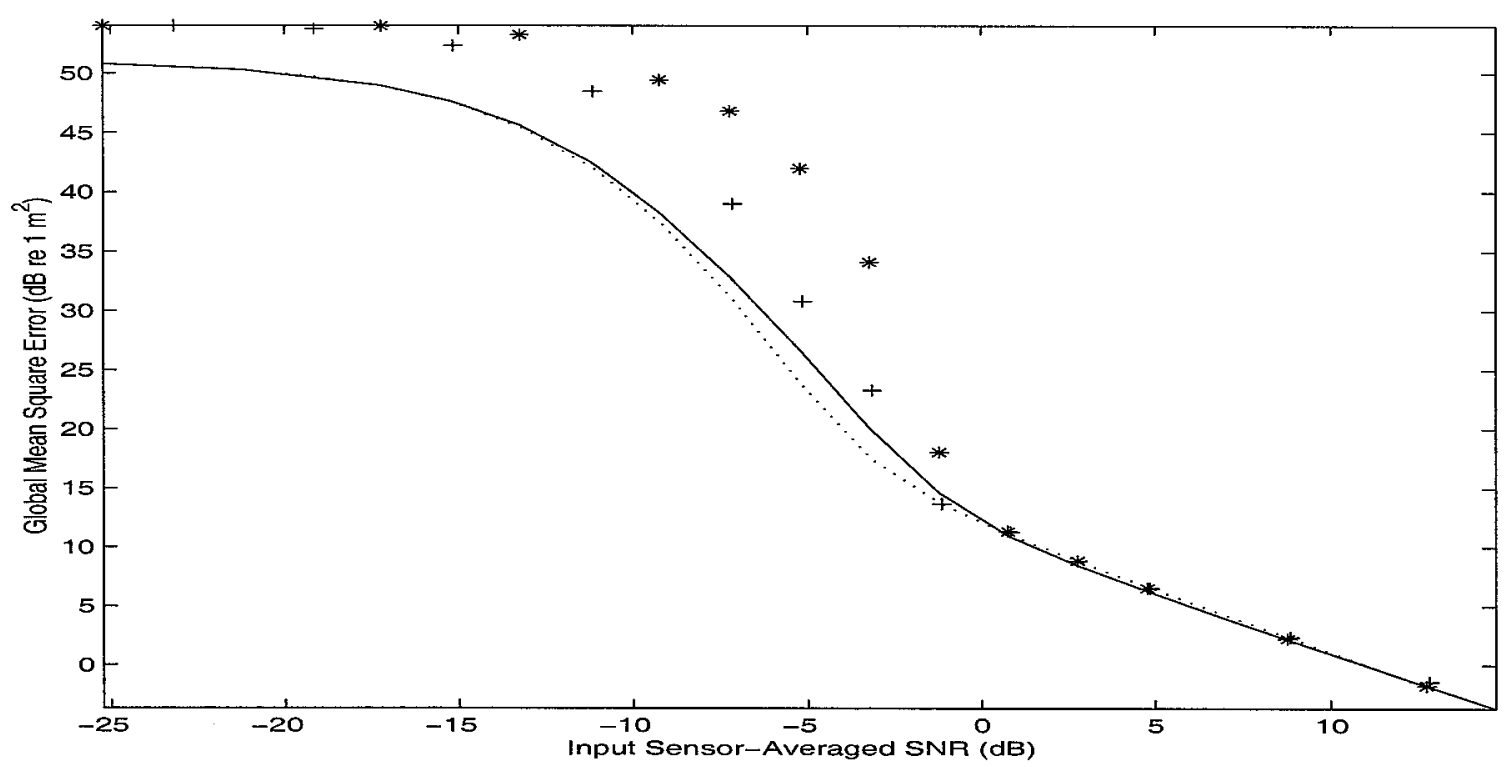

Figure 6-19: Performance bound evaluation for source range estimation. Solid line: ZZB; *: MLE simulations, both with unknown depth; dotted line: ZZB; +: MLE simulations, both with known depth.

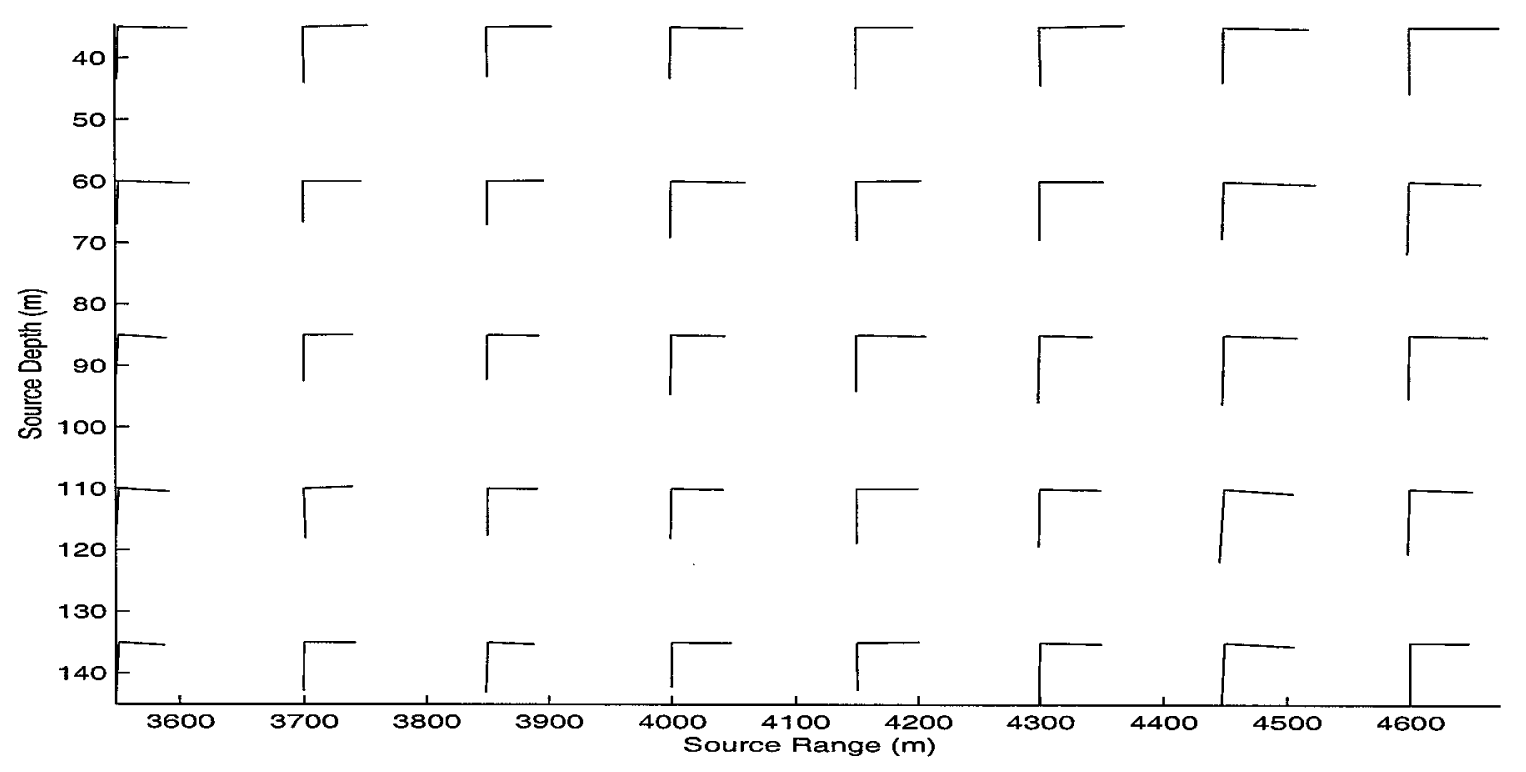

Figure 6-20: Error coupling between source range and depth. The vector pair at each source location denotes the eigenvector set of the resolution matrix specified by the $\mathrm{CRB}$ at $\mathrm{SNR}=15 \mathrm{~dB}$. The length of the eigenvector is the individual resolution amplified by 80 times. 


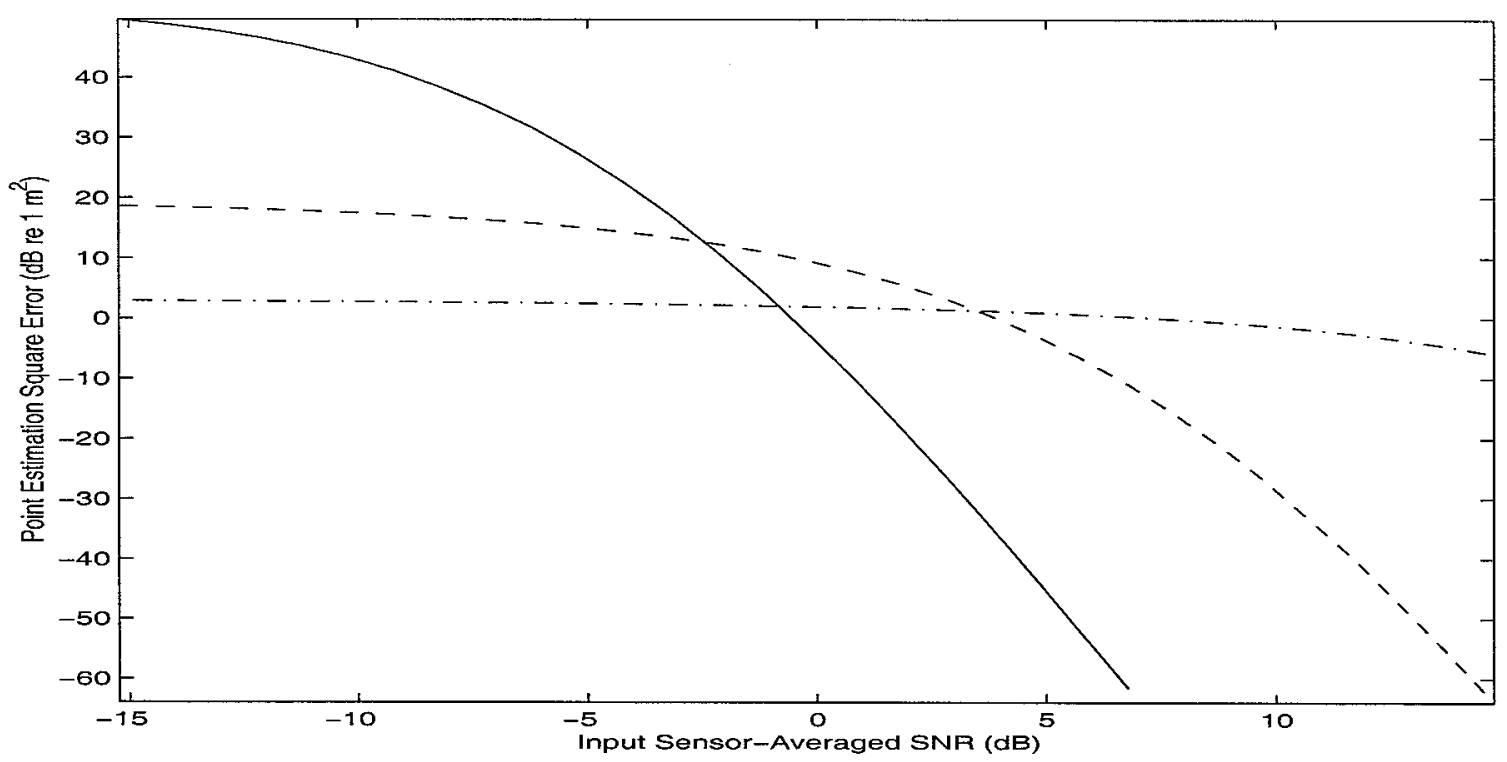

Figure 6-21: Probabilistic square errors as a function of SNR at different projected ambiguity points: close mainlobe point (4123 $\mathrm{m}$, dashdot line); distant mainlobe point (4112 m, dashed line); and distant sidelobe point (4724 m, solid line). The true source location is at $(4125 \mathrm{~m}, 60 \mathrm{~m})$.

in general, deeper (or shallower) the source, less the field ambiguity. This could be attributed to the sound velocity profile in the water column, which switches from downward refracting to isovelocity at the mid-depth. Fig. 6-23 gives the histogram of the threshold SNR predictions. The mean point is $-1 \mathrm{~dB}$ and the 75 -percentile point falls between $0 \mathrm{~dB}$ and $1 \mathrm{~dB}$. These are consistent with the MLE simulations.

Some similar observations are also available for depth estimation with/without range uncertainty, which are not presented.

\subsubsection{Comments on Numerical Computation}

In this example, the parameter sampling step is $0.5 \mathrm{~m}$ for source range, and $0.1 \mathrm{~m}$ for source depth. These are chosen based on the linearity test. Accordingly, we have 2501 (range) $\times 1201$ (depth) parameter points. Even for this two-dimensional problem, direct calculation of the ZZB is rather time-consuming. Some approximations are used per discussions in Section 6.1.

First, the parameter perturbation, $\delta$ in (3.24), is sparsely sampled at the ambiguity 


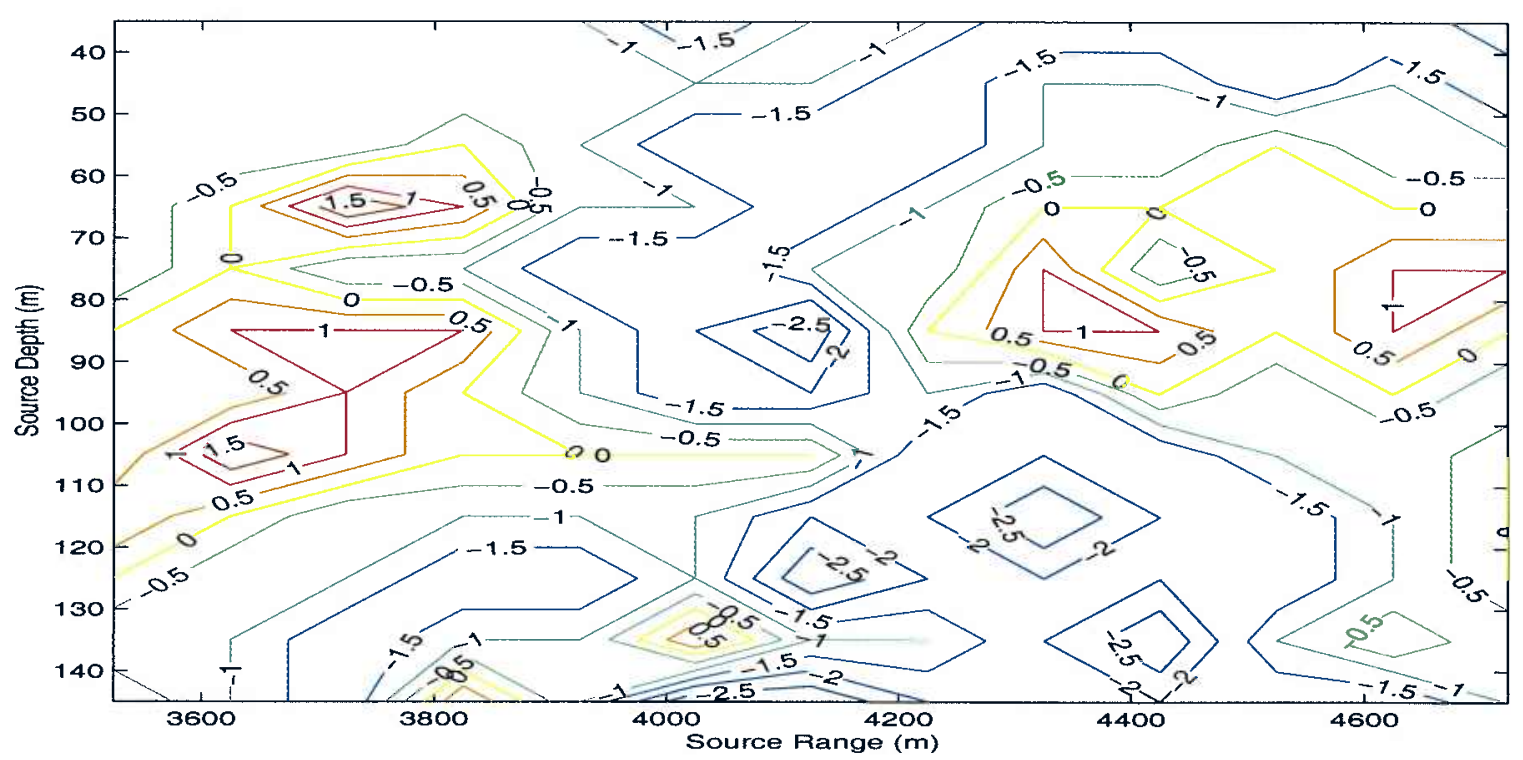

Figure 6-22: Contour of threshold SNR in $\mathrm{dB}$ for range estimation predicted at individual source locations.

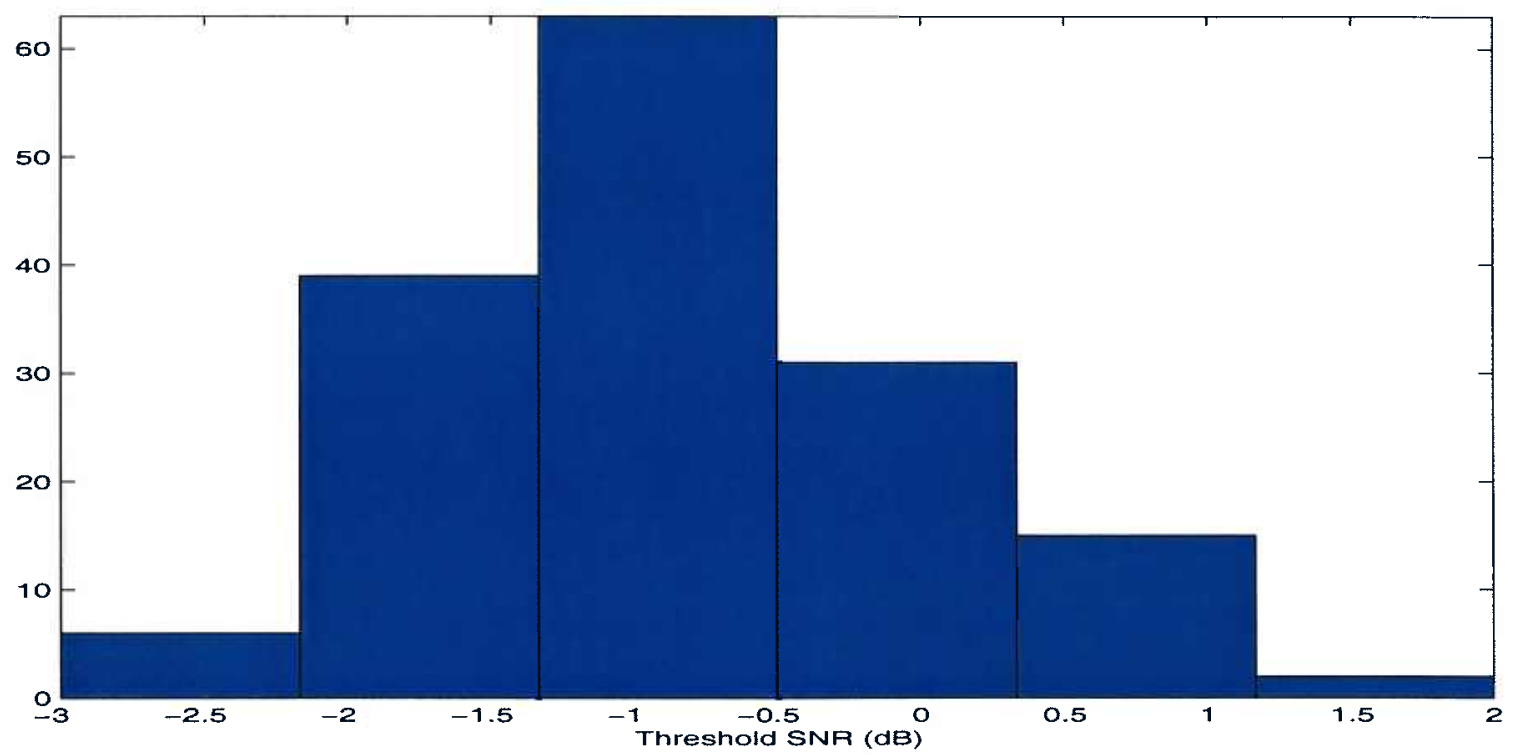

Figure 6-23: Histogram of threshold SNR for range estimation predicted at individual source locations. 
valleys and densely sampled around the mainlobe and sidelobes. As a result, we have 316 perturbation points for source range.

Second, the averaged error probability to be maximized in (3.24)

$$
\int_{\Theta} \min [p(\boldsymbol{\theta}), p(\boldsymbol{\theta}+\boldsymbol{\Delta})] \cdot P_{e}(\boldsymbol{\theta}, \boldsymbol{\theta}+\boldsymbol{\Delta}) d \boldsymbol{\theta}
$$

involves an integration over the parameter space. The numerical integration step is eight times of the parameter sampling step for both range and depth.

Third, Eq. (6.14) under constraint $\mathbf{a}^{T} \Delta=\delta$ (cf. (3.24)) can be a multi-modal function of $\Delta$. In this example, $\Delta$ includes range perturbation and depth perturbation, and we need to find the depth perturbation maximizing (6.14) for each range perturbation $\delta$. Some typical behaviors of this function are plotted in Fig. 6-24. To find the peak of each plot, a local searching algorithm is used. We also notice that for two consecutive range perturbations, peak of (6.14) only shifts slightly. Hence, the local algorithm initially starts from the origin (zero perturbation) and then for the consequent range perturbations, starts from the peak for the last range perturbation. This algorithm works well except for the case of (d). Case (d) corresponds to a large perturbation at high SNR, which obviously can be ignored. At some SNR's around the threshold, the exhausting searching is also implemented, but the improvement is insignificant.

\subsection{Source Range Estimation with Environmental Uncertainty}

In this section, environmental uncertainties are added to the previous source range estimation problem. The uncertain parameters considered are the compressional wavespeeds at top and bottom of the sediment layer, which determine the number of propagation modes in the water column and the fraction of sound penetration reflected back from the sediment. Therefore, these quantities are of the most sensitive parameters for sound waveguide propagation. We assume the sediment top wave-speed is 

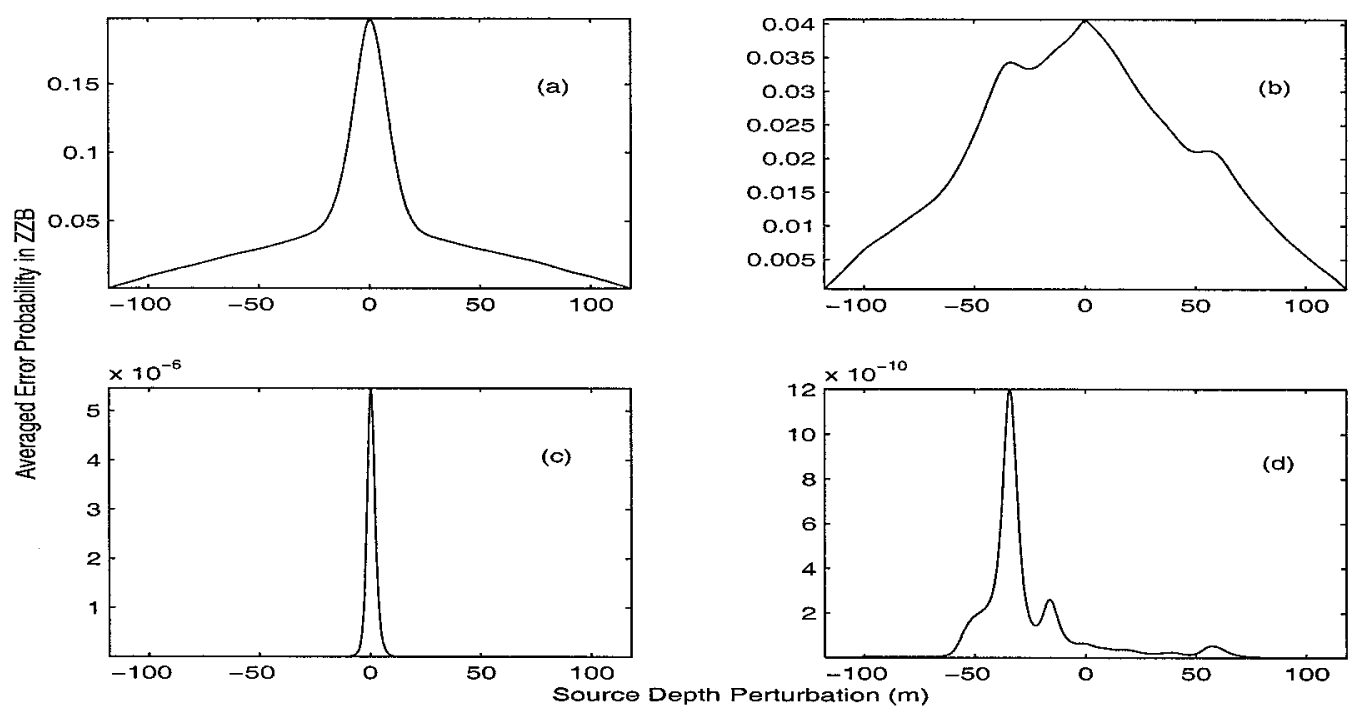

Figure 6-24: Averaged error probability to be maximized in evaluation of the ZZB for source range perturbation of: (a) $36 \mathrm{~m}$; (b) $500 \mathrm{~m}, \mathrm{SNR}=-11 \mathrm{~dB}$; (c) $36 \mathrm{~m}$; (d) $500 \mathrm{~m}, \mathrm{SNR}=5 \mathrm{~dB}$.

uniformly distributed over an interval of $[15501575] \mathrm{m} / \mathrm{s}$ and the sediment bottom wave-speed is uniformly distributed over an interval of $\left[\begin{array}{lll}1575 & 1625\end{array}\right] \mathrm{m} / \mathrm{s}$. Again, the source range is uniformly distributed from $3500 \mathrm{~m}$ to $4750 \mathrm{~m}$.

Similar to the two-dimensional case, evaluation of the bound in a three-dimensional parameter space is computationally intensive and the numerical performance needs to be carefully balanced with the computations. Based on the results of the linearity test, the parameter sampling steps used are $0.5 \mathrm{~m}$ for source range and $1 \mathrm{~m} / \mathrm{s}$ for both sediment wave-speeds. These are also used as the minimum test points of the WWB. Up to 120 test vectors pre-selected around the mainlobe and sidelobes are used in evaluation of the WWB, each of which has a non-zero perturbation only along one of dimensions. At high SNR, only a small close-to-zero subset of test vectors is used to avoid the singularity of the matrix $\mathbf{Q}$ in (3.18). The integration path of the ZZB for source range estimation consists of 316 sampling points within the perturbation interval of $[01250] \mathrm{m}$. This path starts from the sampling step, $0.5 \mathrm{~m}$, and is densely sampled at the mainlobe and sidelobe positions. The integration steps for three parameters are two, four and three times of the corresponding sampling steps, 


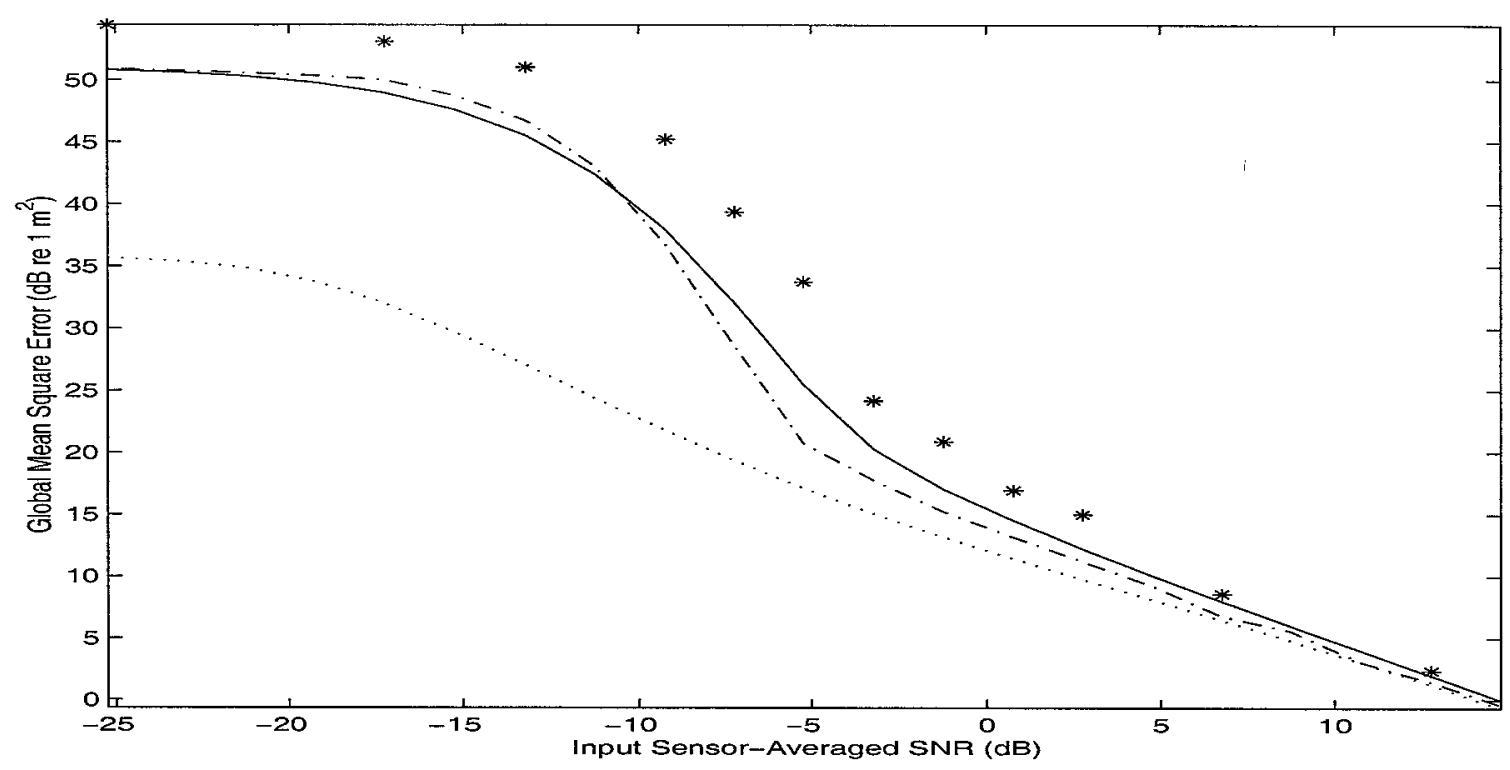

Figure 6-25: Performance bound evaluation for source range estimation with environmental uncertainty. Solid line: ZZB; dashdot line: multiple-test point WWB; dotted line: BCRB; and *: MLE simulations.

respectively. Under these configurations, the evaluation time is several minutes for the BCRB, 10 hours for the ZZB (of source range estimation only) with local searching algorithm, and 24 hours for the WWB without optimization, all using a $600-\mathrm{MHz}$ PC.

Fig. 6-25 gives the evaluation results of the BCRB, WWB and ZZB as well as the MLE simulations for source range estimation. Compared to Fig. 6-1, some similar behaviors are observed. At high SNR, all the three bounds agree with the MLE simulations. Below SNR $=-3 \mathrm{~dB}$, the MLE performance diverges significantly, which is predicted by the WWB and ZZB. At low SNR, both the WWB and ZZB converge to the variance of the a priori parameter distribution. Again, the ZZB is the best bound for most (higher) SNR levels.

Despite those similarities, source localization with environmental uncertainty shows worse performance than that with perfectly known environment, particularly in the intermediate to high SNR region, as shown in Fig. 6-26. For example, at input SNR $=7 \mathrm{~dB}$, the root-mean-square error is about $2.7 \mathrm{~m}$ for the former case versus $1.6 \mathrm{~m}$ for the latter case. At input $\mathrm{SNR}=-1 \mathrm{~dB}$, this is $11 \mathrm{~m}$ versus $4.8 \mathrm{~m}$. 


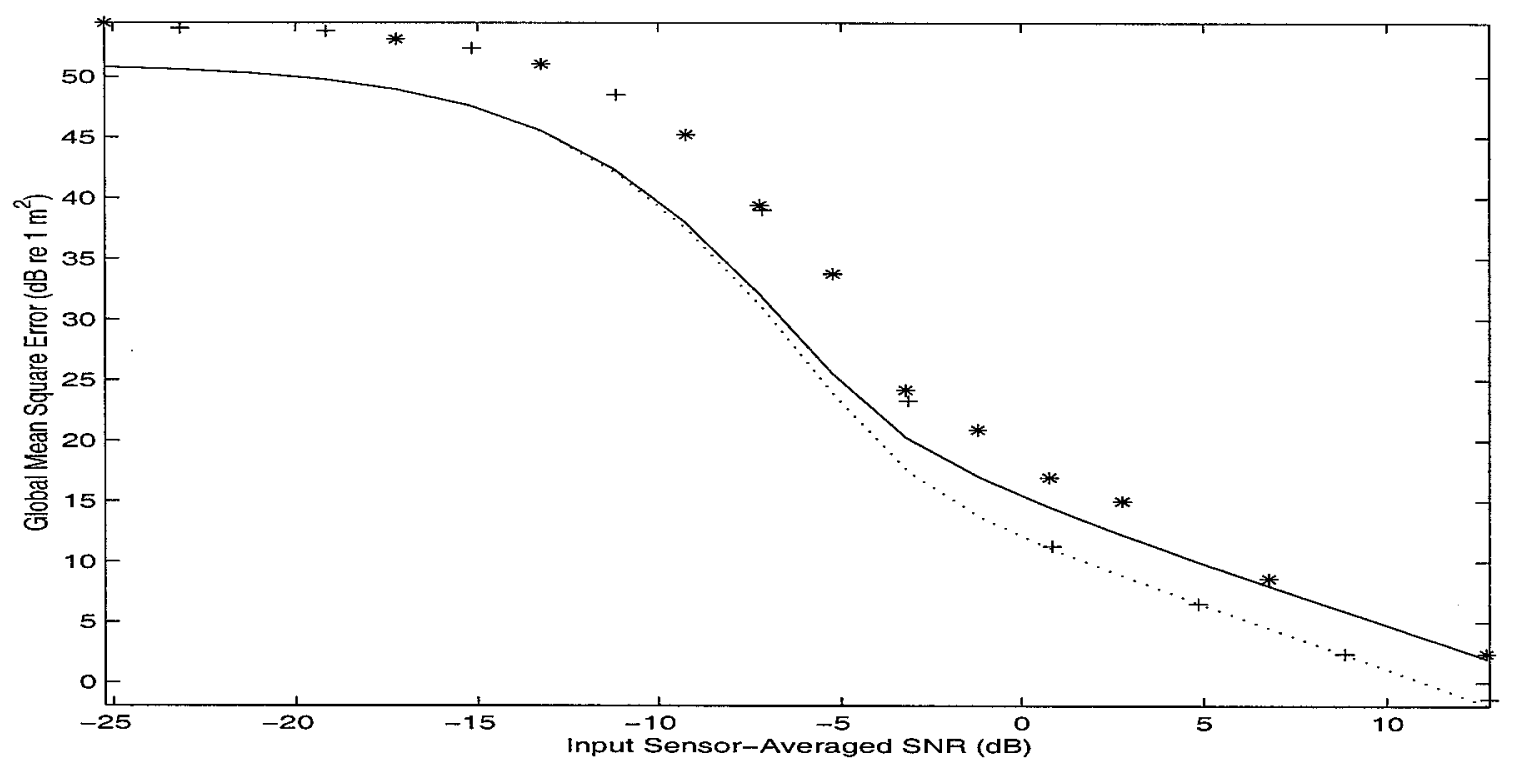

Figure 6-26: Performance bound evaluation for source range estimation. Solid line: ZZB; *: MLE simulations, both with unknown sediment wave-speeds; dotted line: ZZB; +: MLE simulations, both with known sediment wave-speeds.

To explain the difference, let us first look at the ambiguity function in the problem. Fig. 6-27 gives such an example for the given source range of $4125 \mathrm{~m}$, sediment top wave-speed of $1572 \mathrm{~m} / \mathrm{s}$, and bottom wave-speed of $1593 \mathrm{~m} / \mathrm{s}$. The dotted line is the projection onto the range dimension, which, at each scanning range point, is the peak value of the top-bottom wave-speed ambiguity surface. As discussed in Section 4.2 , the source range is strongly coupled to the sediment wave-speeds. This can be seen from the mainlobe shape of the projected ambiguity function, which becomes wider and has increased levels. This strong coupling exists across the entire three-dimensional parameter space (cf. Fig. 6-28). Consequently, we have increased mean square errors at high SNR levels.

The ambiguity behavior can be further analyzed using the two-point estimation error probability. For the given projected ambiguity function, the probabilistic square error at each scanning source range is evaluated and shown in Fig. 6-29(a). The peak value at each SNR specifies the representative ambiguity point, and shifting of such points at different SNR's delimits different operation regions in parameter estimation. Usually, we have three types of representative points: in addition to 


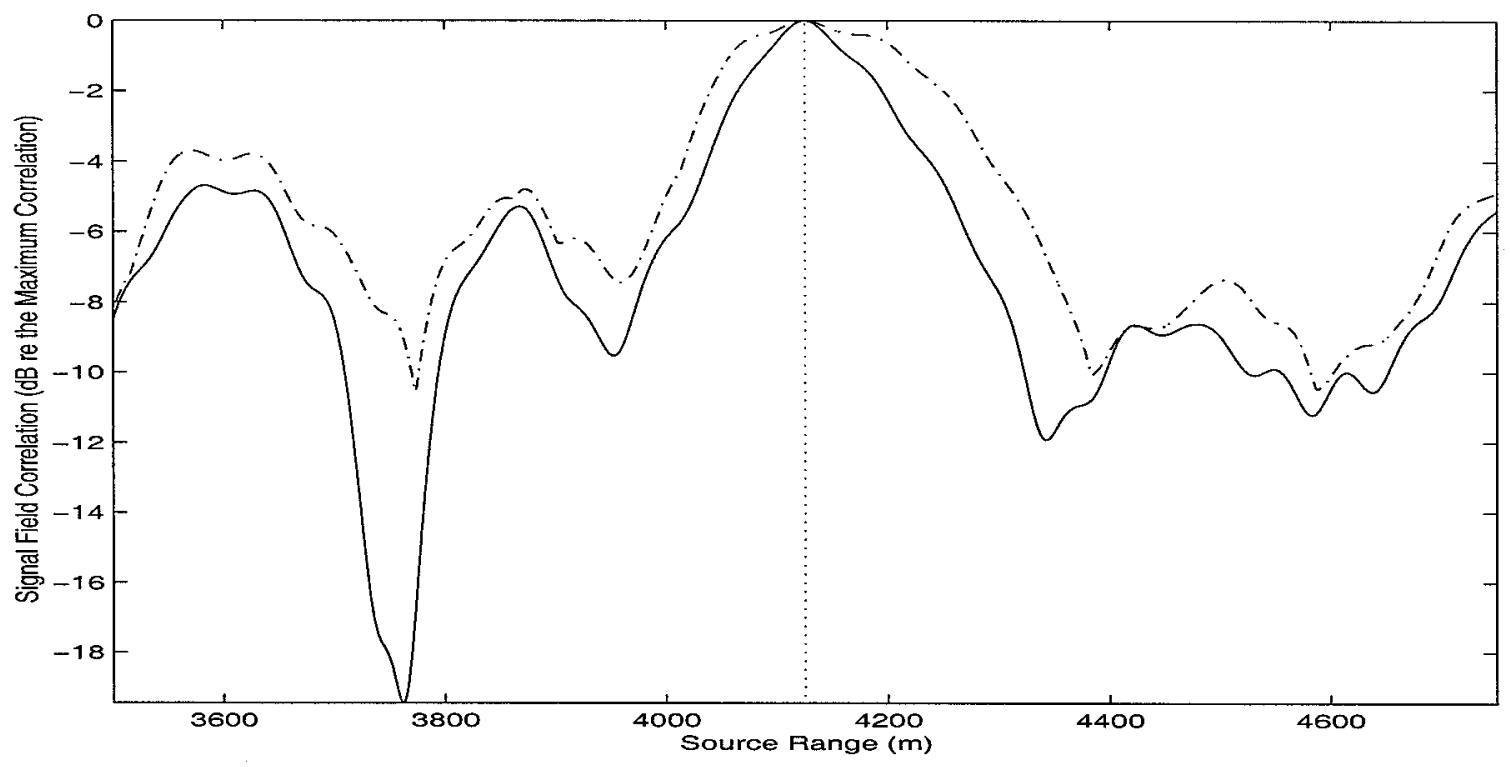

Figure 6-27: Signal ambiguity function for source range estimation with known sediment wave-speeds (solid line) and with unknown sediment wave-speeds (dashdot line). The dotted line indicates the true source range.

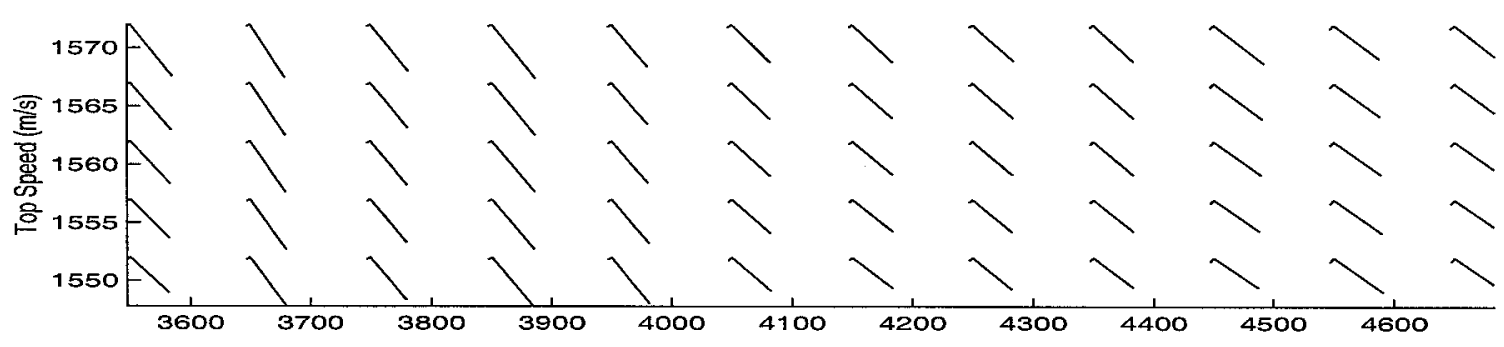

(a)

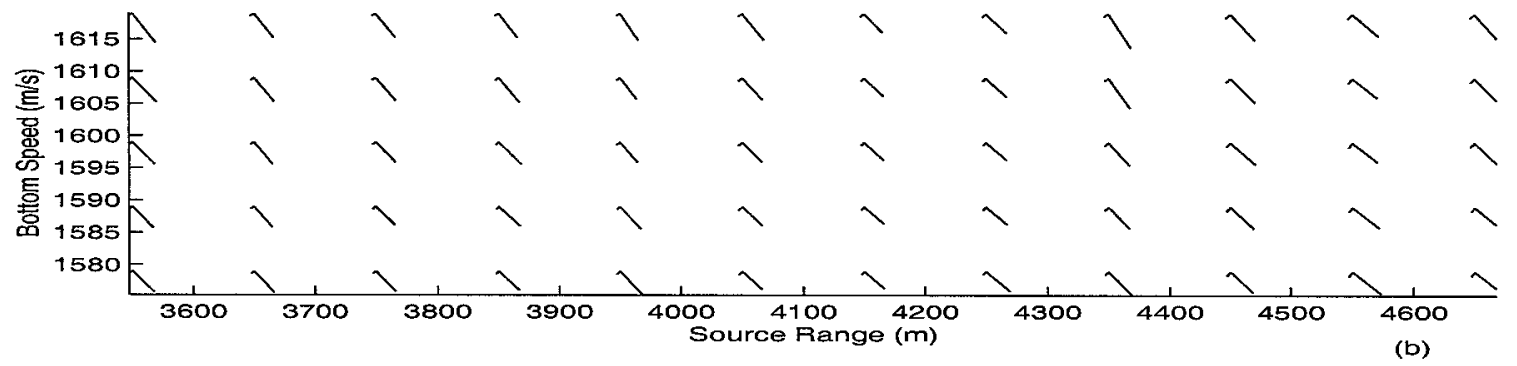

Figure 6-28: Error coupling between source range and sediment wave-speeds: (a) top wave-speed; (b) bottom wave-speed. The vector pair at each parameter location denotes the eigenvector set of the resolution matrix specified by the CRB at SNR = $15 \mathrm{~dB}$. The length of the eigenvector is the individual resolution amplified by 4 times. 
the close-mainlobe points describing the asymptotic behavior and the sidelobe points determining the large performance departure, there are some distant mainlobe points in the middle and the one just before the first dominating sidelobe point is denoted as the representative one. In this example, however, the mainlobe behavior is a little more complicated.

As shown in Fig. 6-29(b), in addition to the regular distant mainlobe point (dashed line), below $\mathrm{SNR}=1 \mathrm{~dB}$, another cluster of mainlobe points, about $42 \mathrm{~m}$ away from the mainlobe peak, contributes most to the mean square error. This actually corresponds to the mainlobe protrusion as seen in Fig. 6-27. Due to relatively increased mainlobe contributions, the predicted threshold SNR is now at about $-5 \mathrm{~dB}$, a bit lower compared to the case with perfectly known environment.

Fig. 6-30 displays the threshold SNR's predicted at a 13 (range) $\times 3$ (sediment top wave-speed) $\times 5$ (sediment bottom wave-speed) subset of parameter points. Each two-dimensional contour is obtained by fixing one parameter and varying the other two. Clearly, the field sidelobe ambiguity is quite uniform at different sediment wavespeeds, particularly for the top one, showing that within the chosen parameter intervals, variations of sediment wave-speeds do not change much the relative mainlobe and sidelobe structure in source range estimation. It is interesting to note that variation of the sidelobe ambiguity follows a coupled way between both sediment wave-speeds. For bottom wave-speed above $1599 \mathrm{~m} / \mathrm{s}$, the coupling is similar to that of the mainlobe case, but below $1599 \mathrm{~m} / \mathrm{s}$, it is opposite. This has not been explained yet.

The mean threshold prediction is $-4 \mathrm{~dB}$ and the 75 -percentile point lies between $-4 \mathrm{~dB}$ and $-3 \mathrm{~dB}$ (Fig. 6-31). These describe the MLE simulations well. The effect of the mainlobe protrusion is also seen from that between $\mathrm{SNR}=-3 \mathrm{~dB}$ and $3 \mathrm{~dB}$, the MLE simulation is 2 to $3 \mathrm{~dB}$ higher than the ZZB, which itself is about 1 to 2 $\mathrm{dB}$ higher than the WWB. This slight threshold behavior is also predicted using the same approach described in Fig. 6-29, and the results are shown in Fig. 6-32. The mean point is $0 \mathrm{~dB}$ and the 75 -percentile point is about $2 \mathrm{~dB}$. Compared to prediction of the sidelobe threshold, the mainlobe one is a bit difficult because it is harder to locate the mainlobe protrusion at some parameter points. 


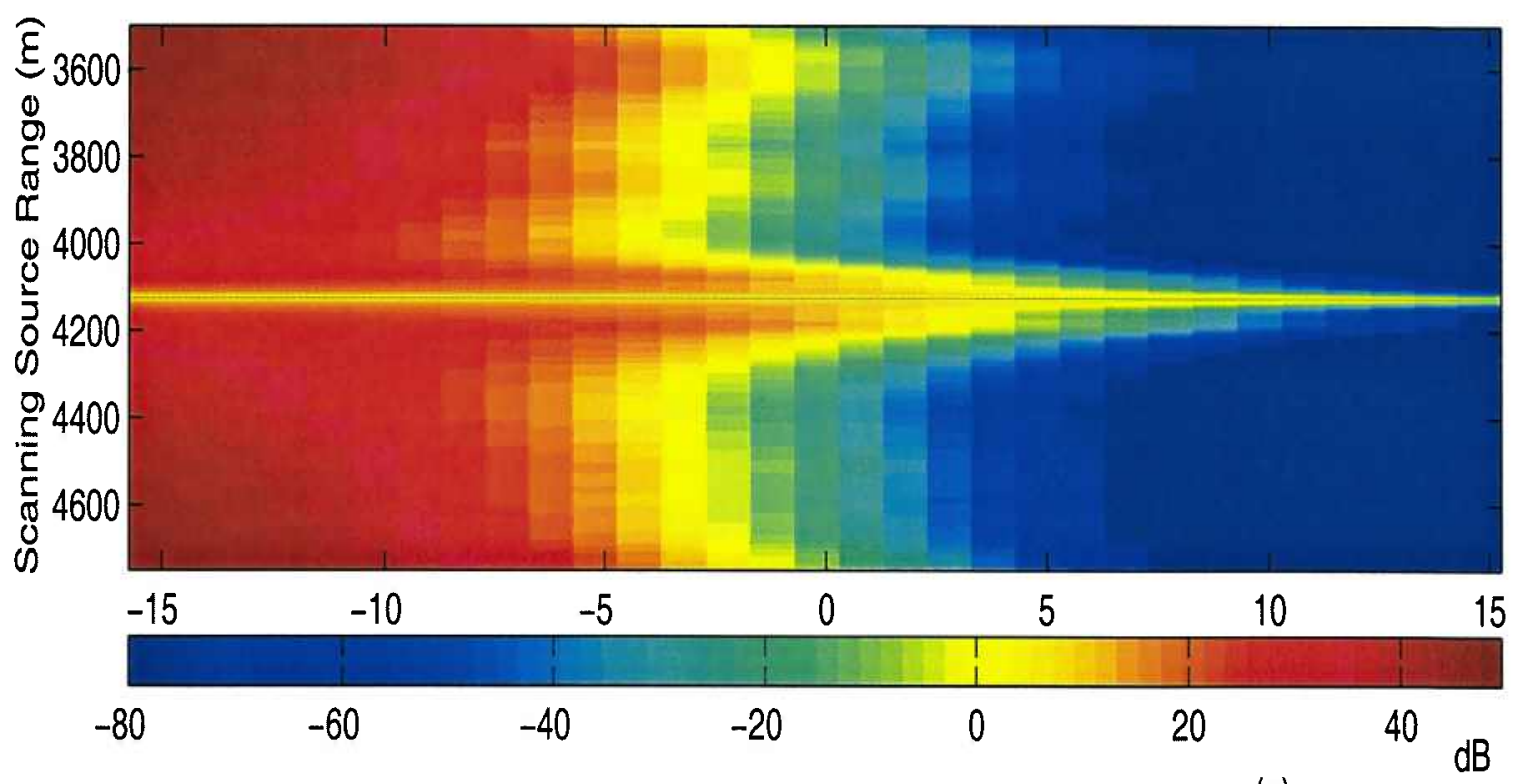

(a)

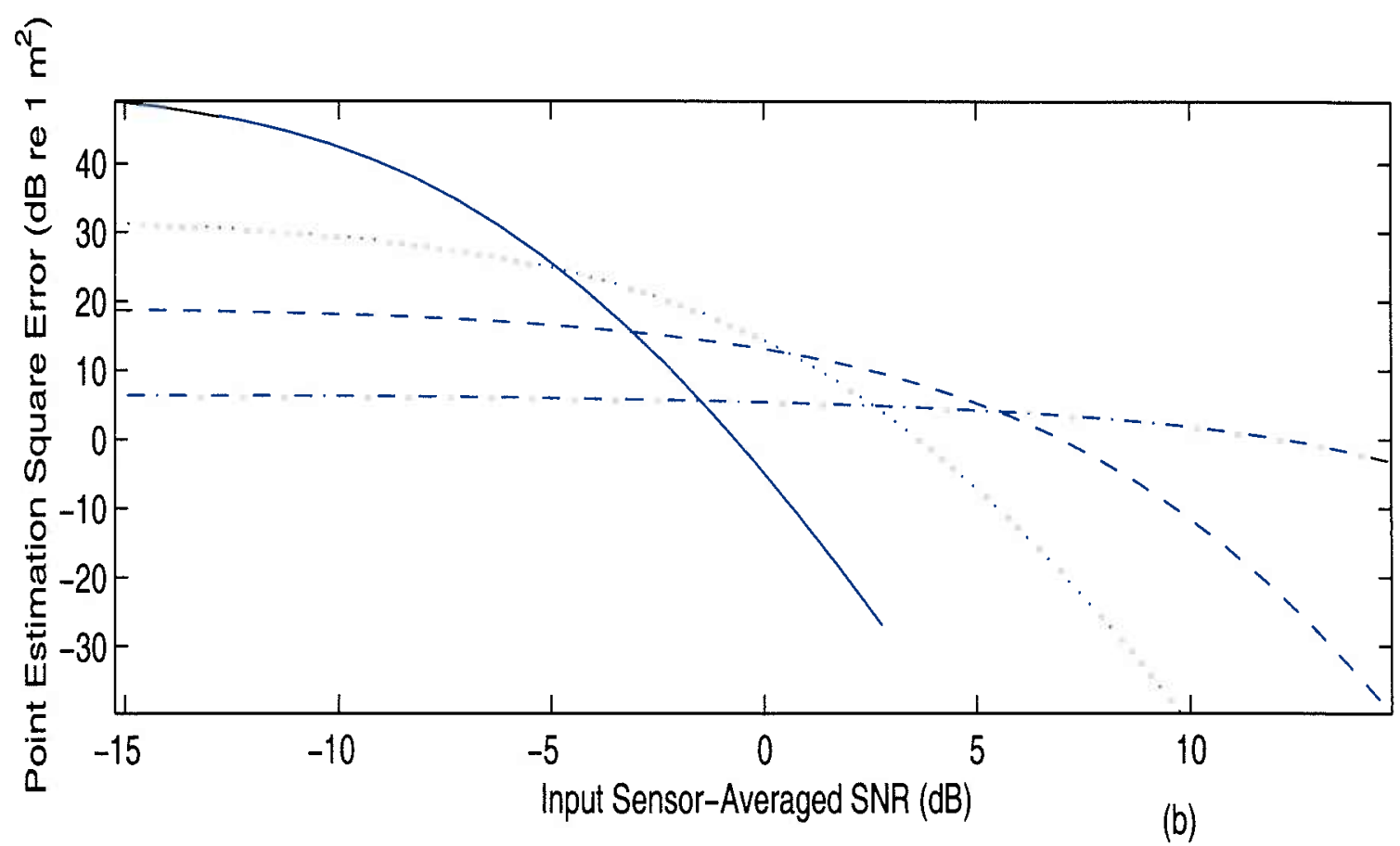

Figure 6-29: Probabilistic square errors as a function of SNR: (a) at each scanning source range (The horizontal line in the middle corresponds to the true source range); (b) at different ambiguity points - close mainlobe point (4122 m, dashdot line), distant mainlobe point (4112 $\mathrm{m}$, dashed line), mainlobe protrusion point $(4184 \mathrm{~m}$, dotted line), and distant sidelobe point (3568 $\mathrm{m}$, solid line). The sediment wave-speeds are assumed unknown. 

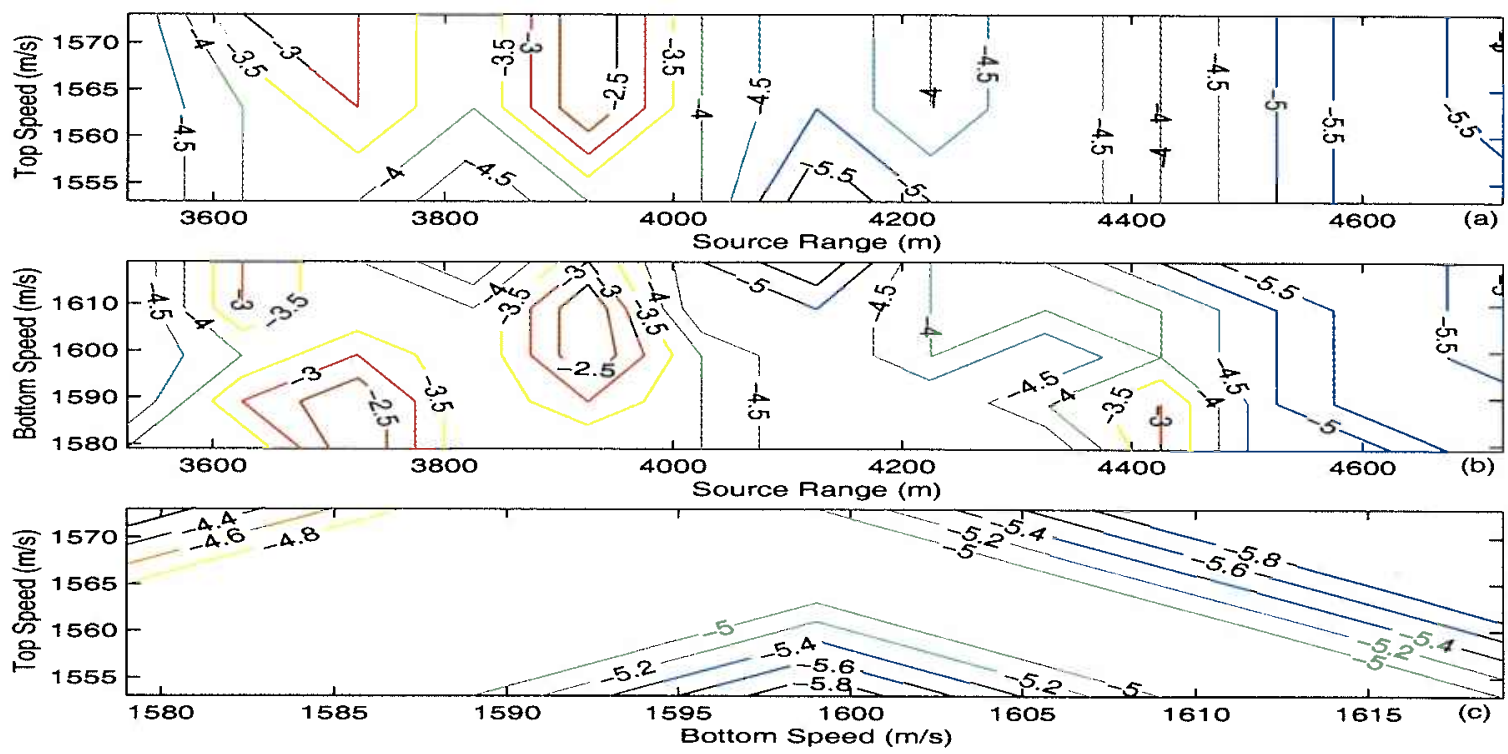

Figure 6-30: Contour of threshold SNR in $\mathrm{dB}$ for range estimation predicted at individual parameter points: (a) sediment bottom wave-speed at $1599 \mathrm{~m} / \mathrm{s}$; (b) sediment top wave-speed at $1563 \mathrm{~m} / \mathrm{s}$; (c) source range at $4125 \mathrm{~m}$.

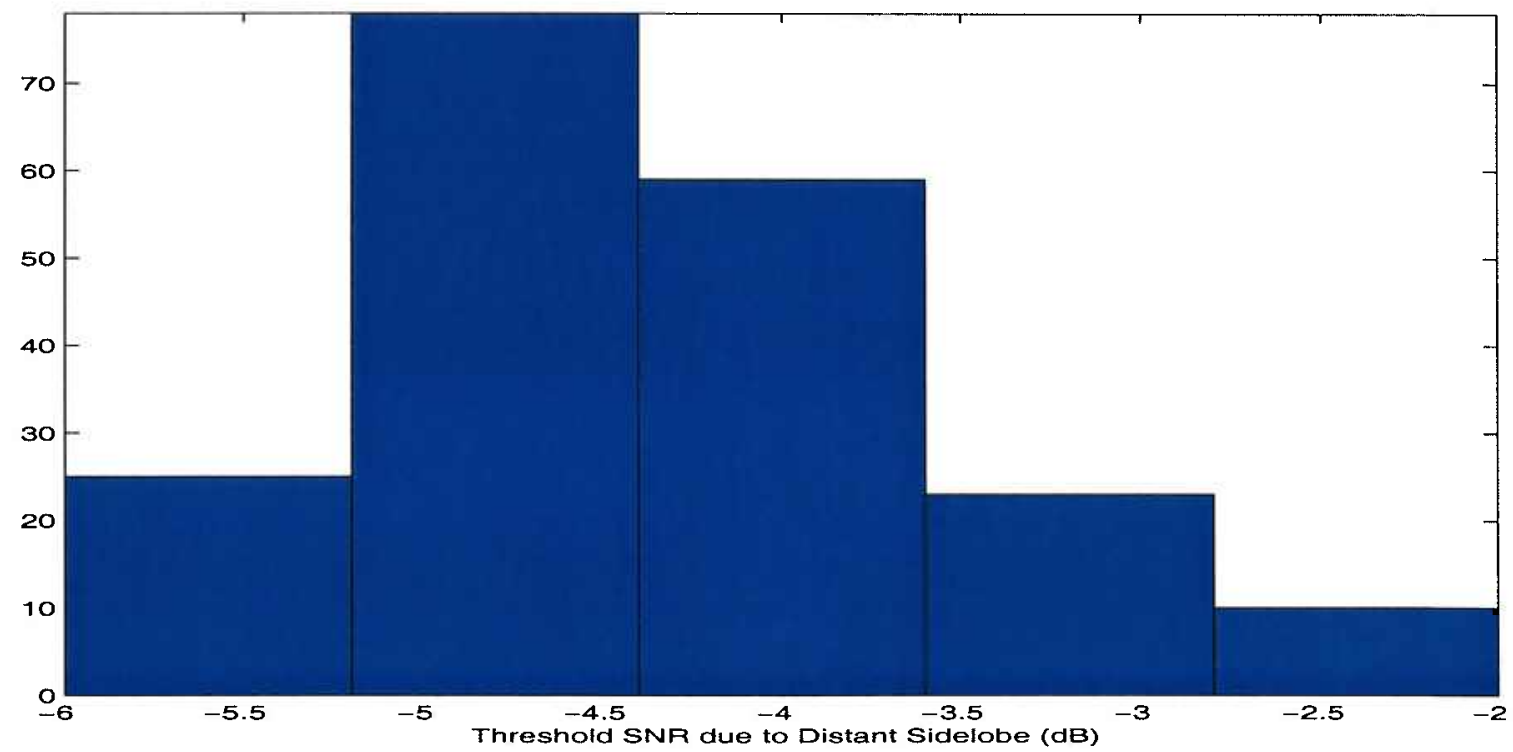

Figure 6-31: Histogram of threshold SNR for range estimation predicted at individual parameter points: sidelobe issue. 


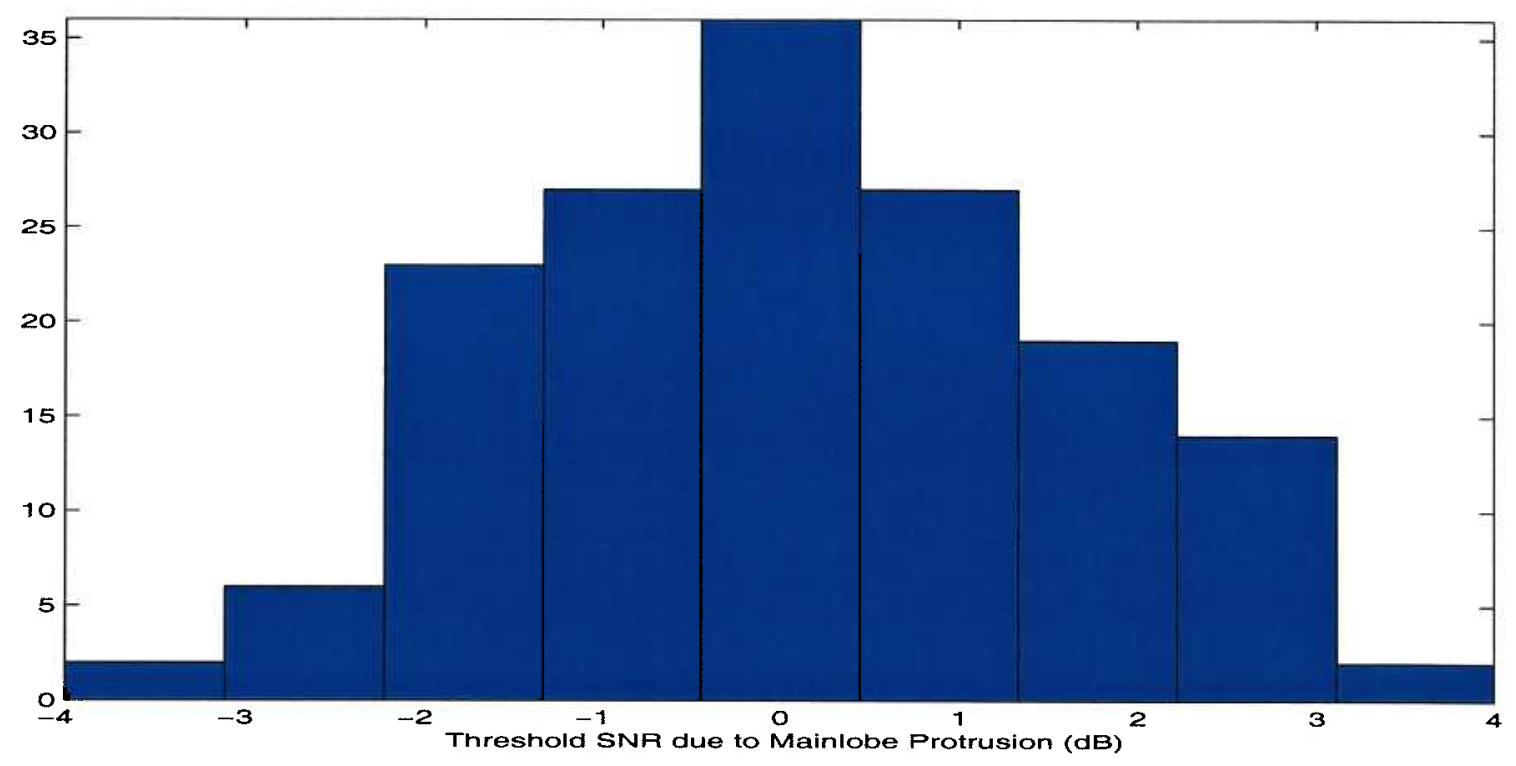

Figure 6-32: Histogram of threshold SNR for range estimation predicted at individual parameter points: mainlobe issue.

Finally, we should point out that the effect of the ambiguity projection is better understood when plotting the average error probability to be maximized in evaluation of the ZZB (cf. (6.14)). Fig. 6-33 shows this averaged error probability as a function of the sediment top and bottom wave-speed perturbations for each chosen source range perturbation ( $\delta$ in (3.24)) at $\mathrm{SNR}=5 \mathrm{~dB}$. Clearly, errors by small range perturbations dominate at this SNR, and for this small range perturbation, the peak averaged error probability is obtained by small sediment top and bottom wave-speed perturbations.

The strong coupling between the top and bottom wave-speeds is also observed in Fig. 6-33. It is often desired by some adaptive searching algorithms to establish a decoupled parameter set. For example, in evaluation of the ZZB, we need to find the maximum for each of plots in Fig. 6-33. Fortunately, they are unimodal, and a gradient-based algorithm works well. Nonetheless, if these plots are multi-modal and the parameters are strongly coupled (as they are in this problem), an adaptive algorithm may converge on a wrong local maximum. In this case, a global optimization algorithm may be appropriate. 


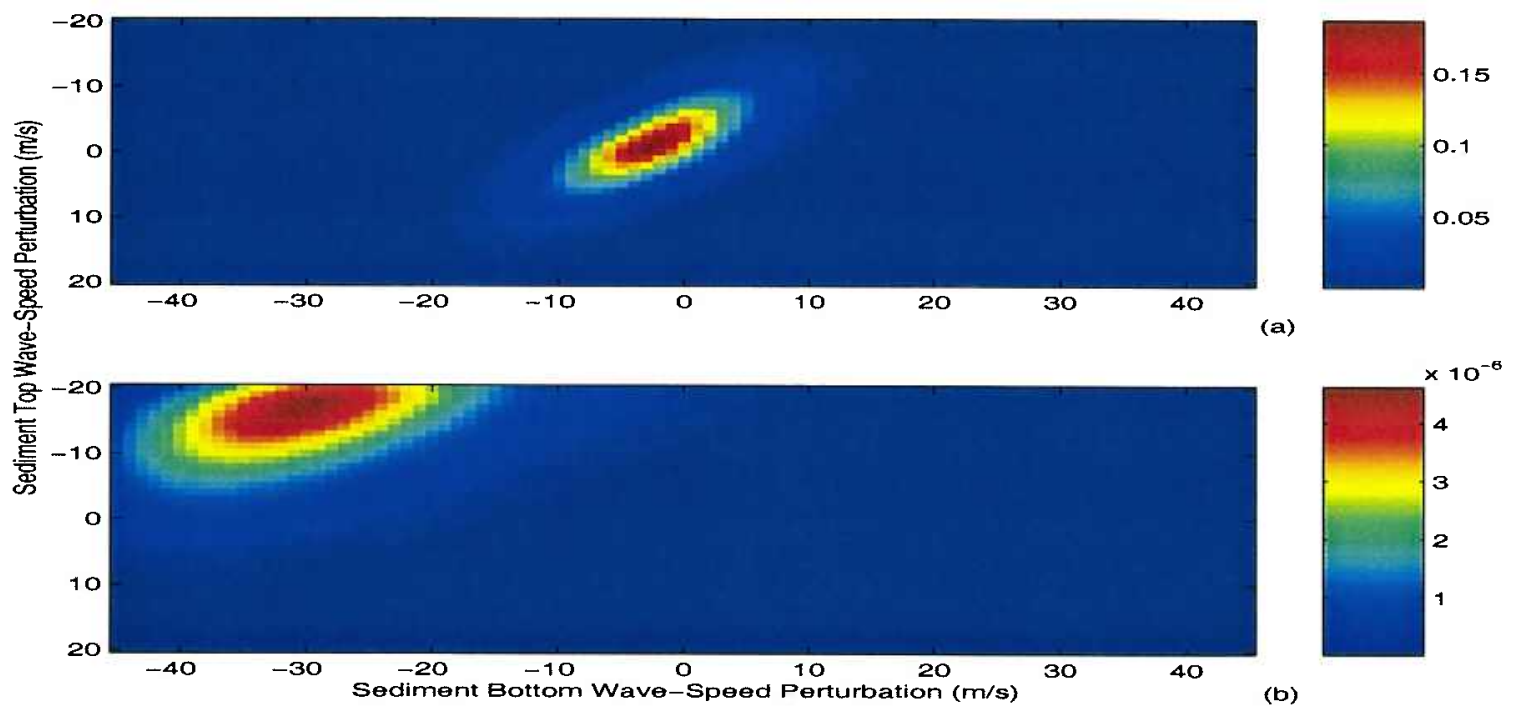

Figure 6-33: Averaged error probability to be maximized in evaluation of the ZZB for source range perturbation of: (a) $5 \mathrm{~m}$; (b) $116 \mathrm{~m}$, both at $\mathrm{SNR}=5 \mathrm{~dB}$.

\subsection{Source Range Estimation with Environmental}

\section{Mismatch}

In Section 5.3, we have presented an example of mismatch analysis in bearing estimation using incorrect array tilt angle. Mismatch analysis in the matched-field problem can be more complicated, particularly when involving many parameters. In that case, behaviors (individual and interactive) of the related ambiguity functions in (5.24), (5.25) and (5.26) can be hard to predict. However, as long as the size of mismatch is within a reasonable range (for example, parameter interval assumed for a regular inversion algorithm) so that the relative mainlobe and sidelobe ambiguity structure is maintained, the performance results are well understandable.

Fig. 6-34 shows such an example for source range estimation under the studied SWellEX-3 environment, in which the assumed sediment wave-speeds are $1572 \mathrm{~m} / \mathrm{s}$ on the top and $1593 \mathrm{~m} / \mathrm{s}$ on the bottom. The top panel is for true wave-speeds of $1565 \mathrm{~m} / \mathrm{s}$ (top) and $1600 \mathrm{~m} / \mathrm{s}$ (bottom), and the bottom panel is for $1550 \mathrm{~m} / \mathrm{s}$ (top) and $1625 \mathrm{~m} / \mathrm{s}$ (bottom). Comparing Fig. 6-34 to Fig. 6-1, we see that with a slight mismatch, the mean square error at high SNR shows a slight increase, for example, 


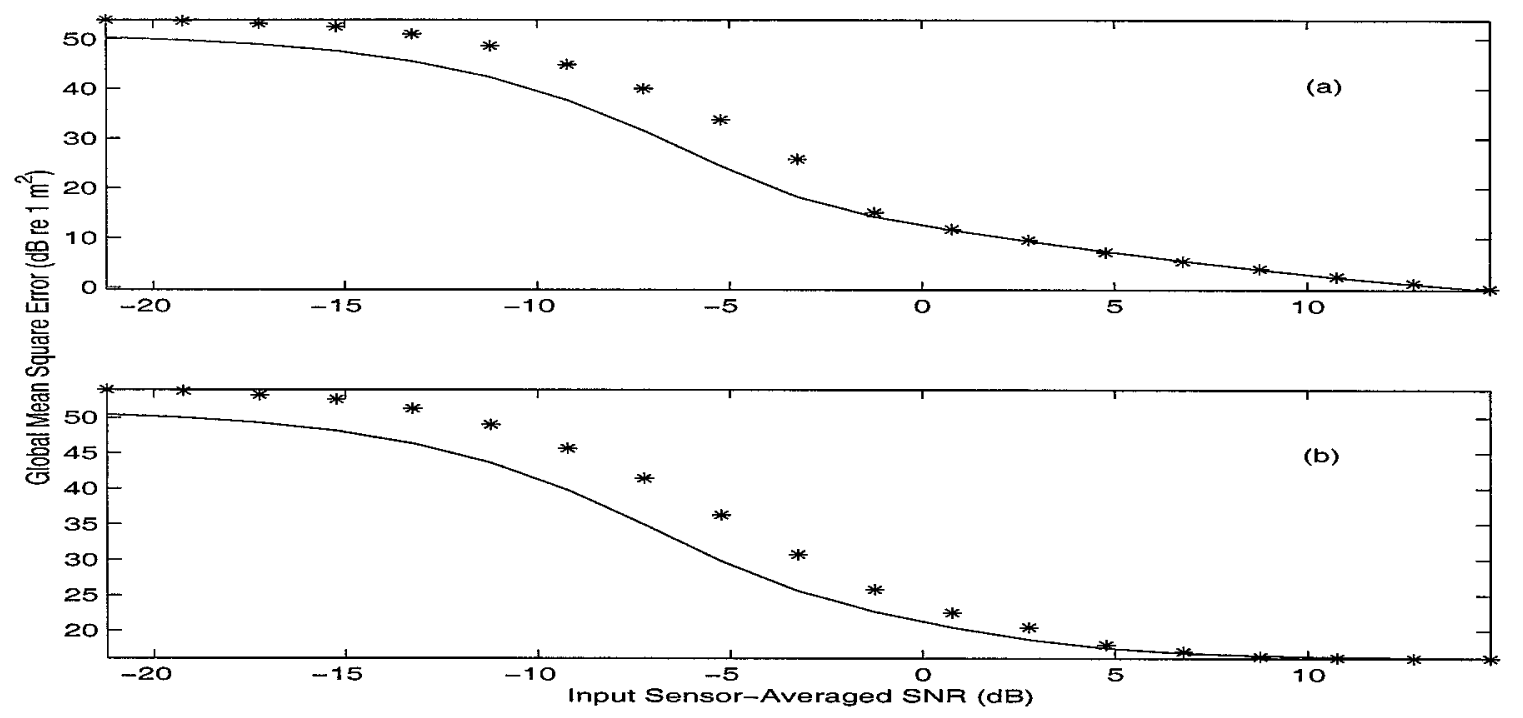

Figure 6-34: Performance bound evaluation for source range estimation with environmental mismatch: ZZB (solid line) versus MLE $\left({ }^{*}\right)$. True sediment wave-speeds are (a) $1565 \mathrm{~m} / \mathrm{s}$ (top) and $1600 \mathrm{~m} / \mathrm{s}$ (bottom); (b) $1550 \mathrm{~m} / \mathrm{s} \mathrm{(top)} \mathrm{and} 1625 \mathrm{~m} / \mathrm{s}$ (bottom). The assumed wave-speeds are $1572 \mathrm{~m} / \mathrm{s}$ (top) and $1593 \mathrm{~m} / \mathrm{s}$ (bottom).

from $1.6^{2} \mathrm{~m}^{2}$ to $1.9^{2} \mathrm{~m}^{2}$ at $\mathrm{SNR}=7 \mathrm{~dB}$; with further increased mismatch, the mean square error is almost independent of the SNR in the high SNR region.

The probabilistic square errors at each scanning source range point as well as representative ambiguity range points given the true point at $4125 \mathrm{~m}$ are displayed in Figs. 6-35 and 6-36. We can clearly see the appearance of a mainlobe protrusion as well as sidelobes. As shown in Fig. 5-1, the major sidelobe level is a little lower, which, combined with the increased mainlobe level, results in a lower sidelobe threshold ($5 \mathrm{~dB}$ versus $-3 \mathrm{~dB}$ in Fig. 4-18). Nonetheless, the mainlobe protrusion makes the performance enter the threshold region earlier.

An observation different from the previous cases is that there is a narrow strip of strong error right on the right side of the true point. This actually corresponds to the region described in Section 5.2.2, in which the mismatched field is more correlated with the true field in contrast to the mismatched field at the true source position. Indeed, range points in this region dominate the error above $\mathrm{SNR}_{M}$ and lead to a bias term in range estimation. The existence of the bias term is verified from the MLE simulations, as shown in Fig. 6-37. Above $\mathrm{SNR} \approx 5 \mathrm{~dB}$, there is nearly a constant 


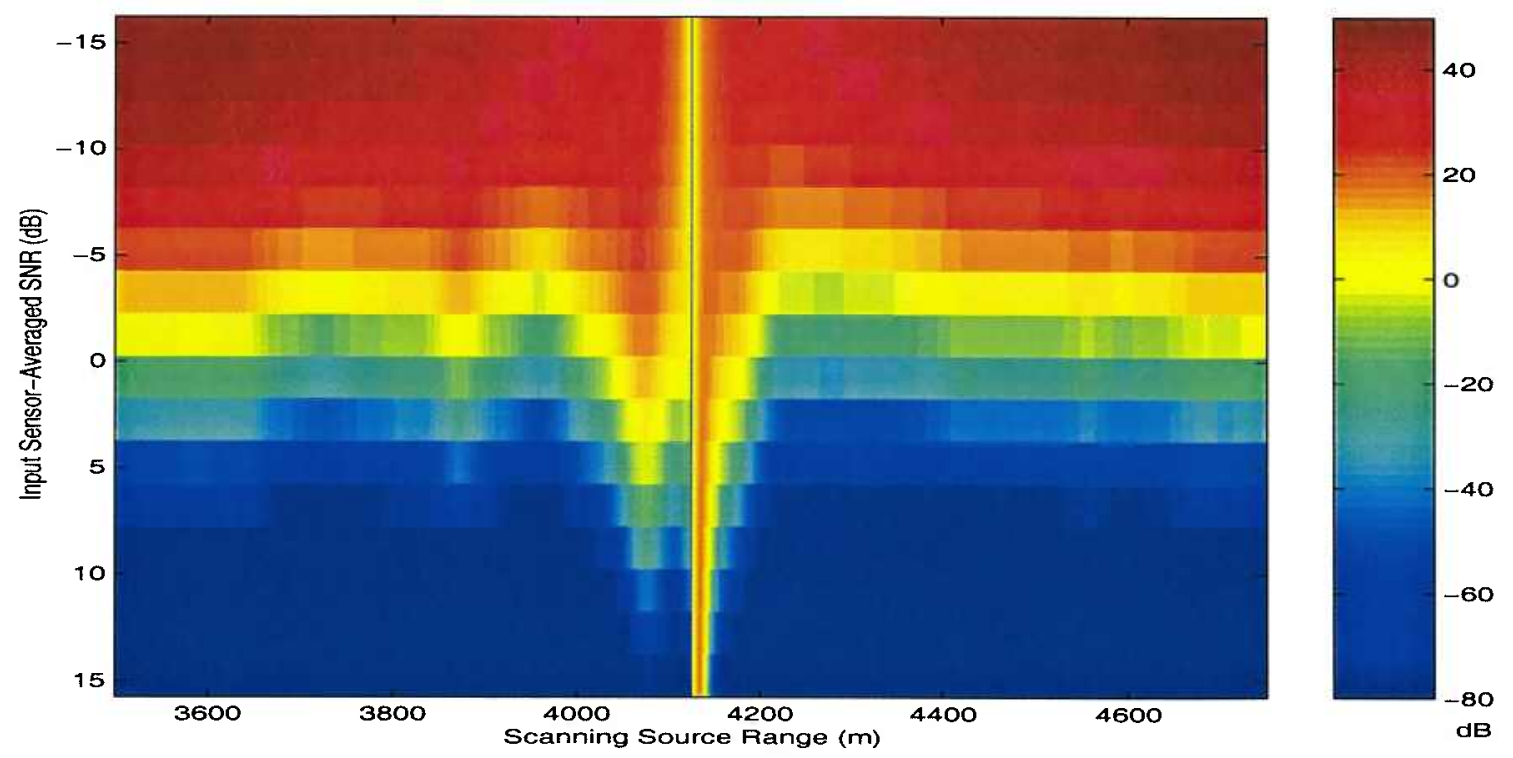

Figure 6-35: Probabilistic square error as a function of SNR at each scanning source range. The sediment wave-speeds are mismatched as given in Fig. 6-34(b). The vertical line in the middle corresponds to the true source range.

bias of $5.7 \mathrm{~m}$.

As mentioned in Section 5.3, the mismatch-introduced bias can be estimated using the MZZB (cf. (5.49)). The square-root of the MZZB at SNR $=15 \mathrm{~dB}$ gives an estimate of $6.4 \mathrm{~m}$. When evaluated at higher SNR's, this estimate should be more accurate since the variance is further decreased. The bias term can also be evaluated by implementing the perturbation integration of the ZZB (cf. (5.10)) over the mismatch-dominated region only.

There are many other applications using the developed mismatch analysis. For example, one may concern, for a given parameter, how the bias term varies in the presence of different sizes of mismatch. Back to the previous range estimation problem, we now assume $0.5 \%, 1 \%, 2 \%, 3 \%, 4 \%$, and $5 \%$ mismatch for the sediment top wave-speed, respectively. At SNR $=11 \mathrm{~dB}$, the resolution goes from $1 \mathrm{~m}$ at the no-mismatch case to about $192 \mathrm{~m}$ with $5 \%$ mismatch, as shown in Fig. 6-38. When generalized to include multiple parameters, the mismatch is not simply accumulated. Indeed, as discussed before, some parameters can be strongly coupled; accordingly, their mismatch effects are coupled as well. Fig. 6-39 shows a contour of bias when 


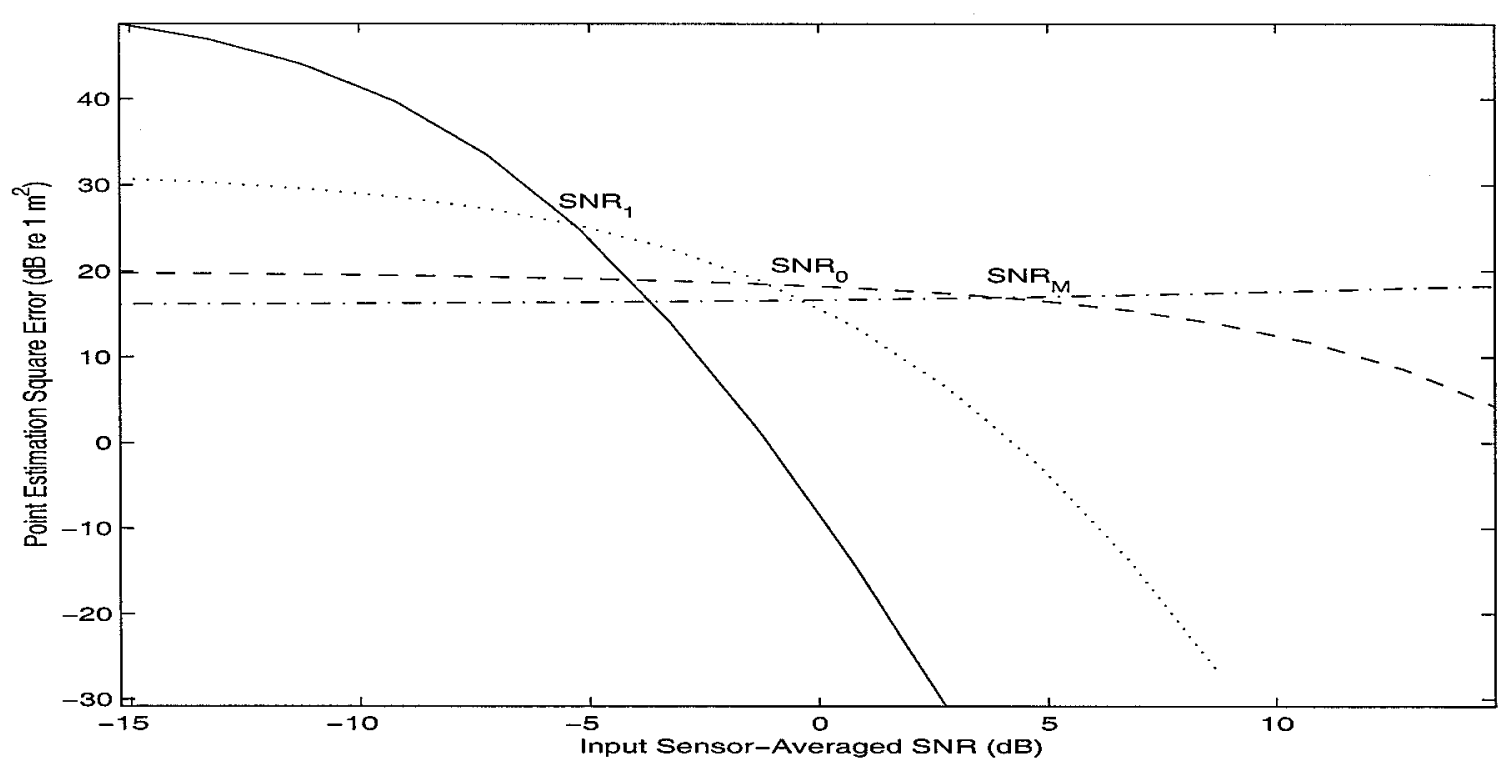

Figure 6-36: Probabilistic square errors as a function of SNR at different ambiguity points: close mainlobe point ( $4134 \mathrm{~m}$, dashdot line); distant mainlobe point (4139 m, dashed line); mainlobe protrusion point (4071 m, dotted line); and distant sidelobe point ( $3579.5 \mathrm{~m}$, solid line). The sediment wave-speeds are mismatched as given in Fig. 6-34(b). The true source range is at $4125 \mathrm{~m}$.

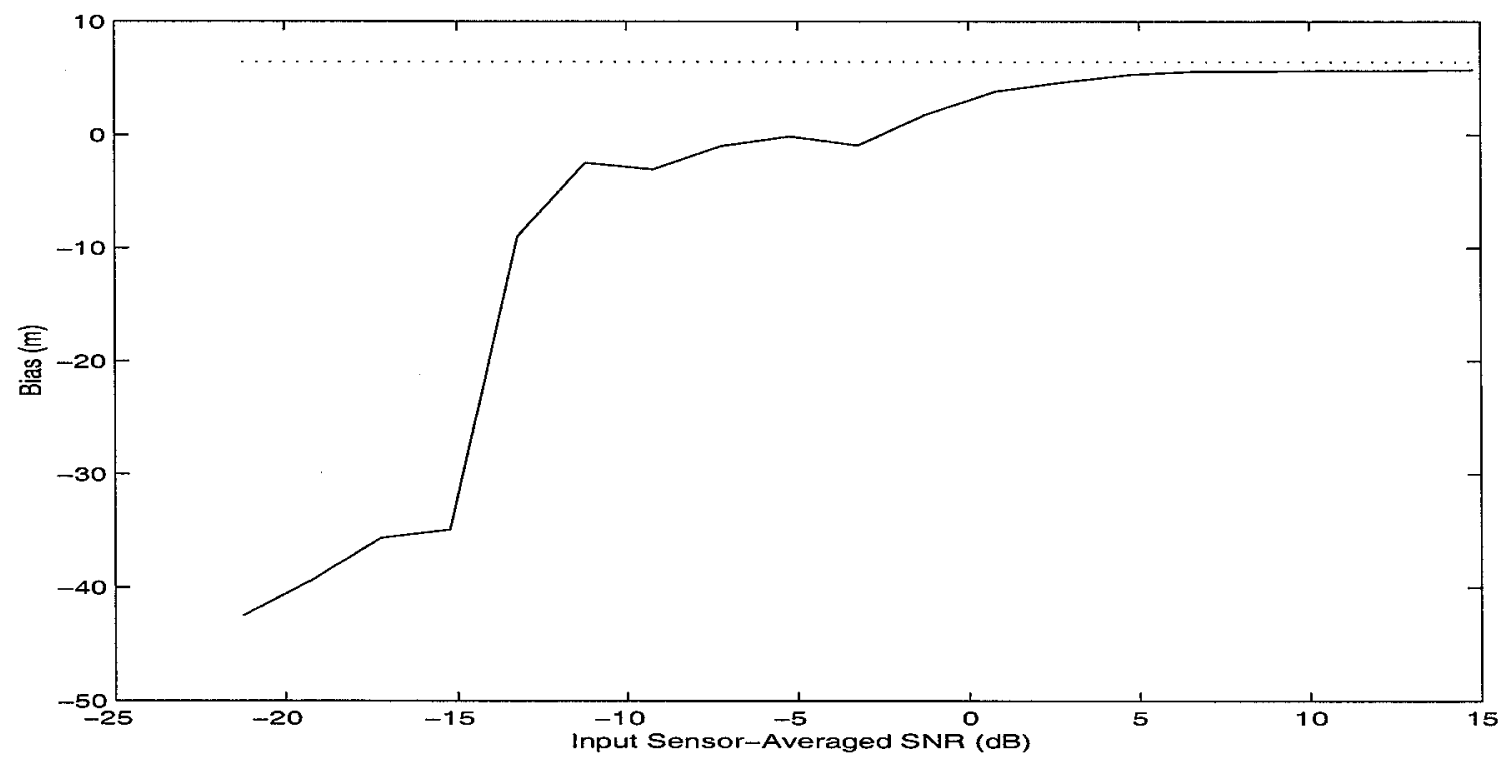

Figure 6-37: Bias in source range estimation. The sediment wave-speeds are mismatched as given in Fig. 6-34(b). The dotted line is the square-root of the ZZB at $\mathrm{SNR}=15 \mathrm{~dB}$. 


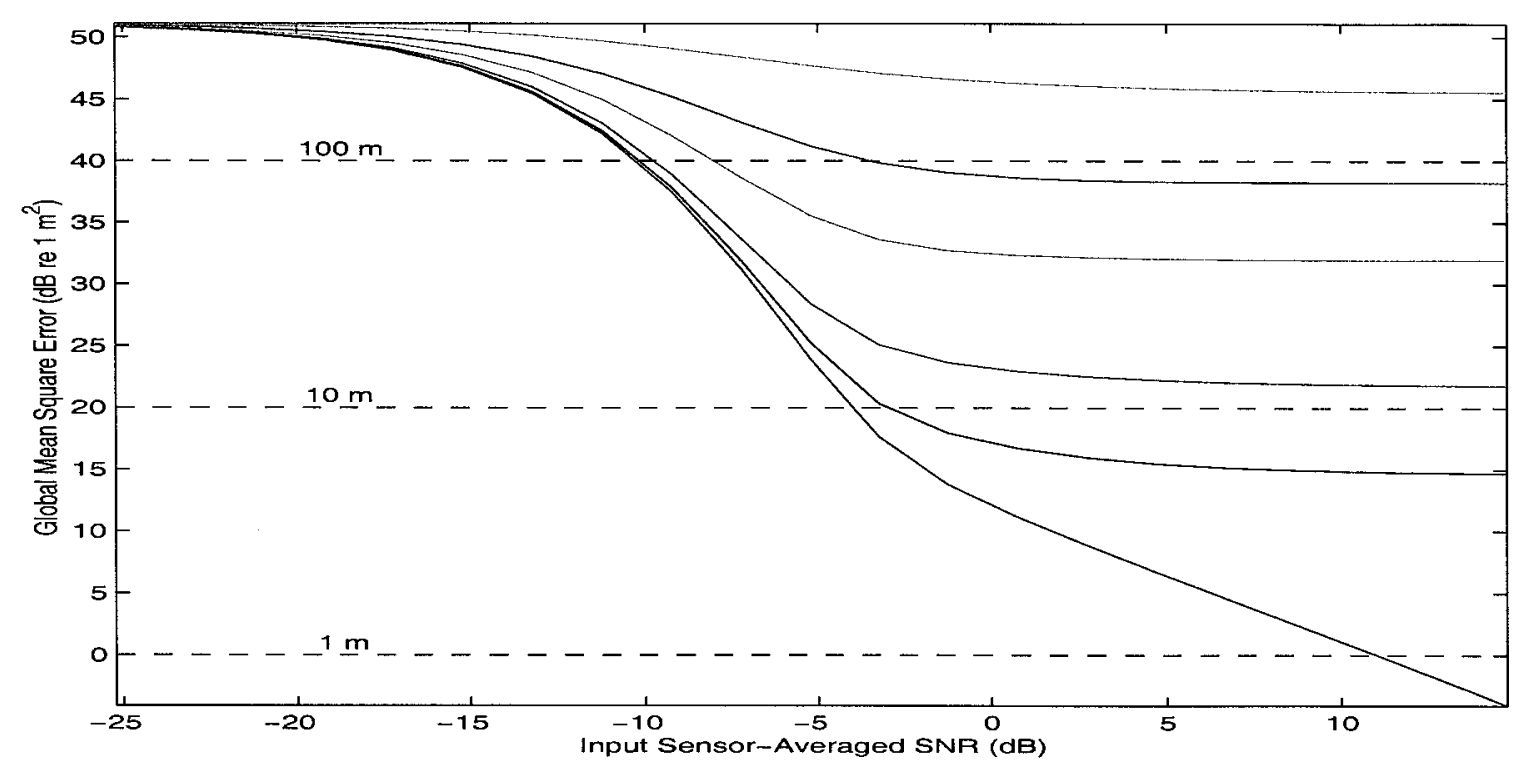

Figure 6-38: Performance bound evaluation for source range estimation at different true sediment top wave-speeds. Bottom to top: $1572 \mathrm{~m} / \mathrm{s} ; 1564 \mathrm{~m} / \mathrm{s} ; 1556 \mathrm{~m} / \mathrm{s} ; 1541$ $\mathrm{m} / \mathrm{s} ; 1526 \mathrm{~m} / \mathrm{s} ; 1512 \mathrm{~m} / \mathrm{s}$; and $1497 \mathrm{~m} / \mathrm{s}$. The assumed top wave-speed is $1572 \mathrm{~m} / \mathrm{s}$.

the true sediment wave-speeds vary from $1550 \mathrm{~m} / \mathrm{s}$ to $1575 \mathrm{~m} / \mathrm{s}$ (top) and from 1575 $\mathrm{m} / \mathrm{s}$ to $1625 \mathrm{~m} / \mathrm{s}$ (bottom). For the true top wave-speed at $1550 \mathrm{~m} / \mathrm{s}$, if the true bottom wave-speed goes from the assumed $1593 \mathrm{~m} / \mathrm{s}$ to the low end, i.e., $1575 \mathrm{~m} / \mathrm{s}$, the overall bias is further increased; if, however, the true bottom wave-speed goes to the high end, say $1620 \mathrm{~m} / \mathrm{s}$, the introduced bias is significantly reduced.

Mismatch analysis can also be used to quantitatively investigate the importance of individual parameters. For example, we assume one percent mismatch for each of the sediment properties, and then evaluate the MZZB for each case. If the bias introduced by one parameter mismatch is large, knowledge about this parameter is important in determining the source location. As shown in Fig. 6-40, it is usually true that deeper the sediment property, less influential to the field. Compared to the no-mismatch case (bottom line in Fig. 6-40), we can hardly tell the effects of the density and attenuation in each layer and the wave-speed in the basement. One exception is the top wave-speed of the mudstone layer, which introduces a larger bias than that by the thickness of the sediment layer. Indeed, this top wave-speed is critical in determining the sound penetration reflected back to the water column. 


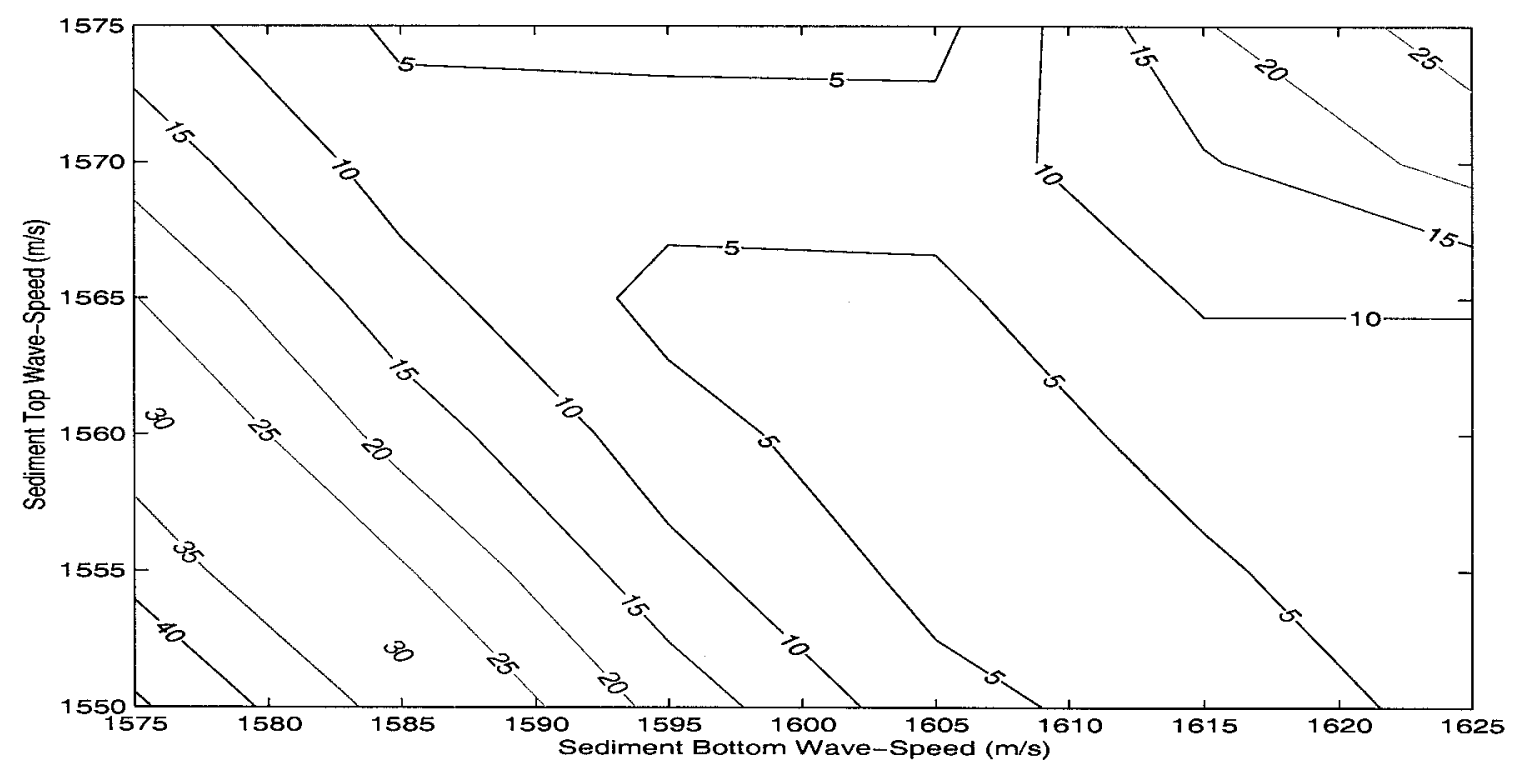

Figure 6-39: Contour of bias in meter in source range estimation. True sediment wave-speeds range from $1550 \mathrm{~m} / \mathrm{s}$ to $1575 \mathrm{~m} / \mathrm{s}$ (top) and from $1575 \mathrm{~m} / \mathrm{s}$ to $1625 \mathrm{~m} / \mathrm{s}$ (bottom). The assumed wave-speeds are $1572 \mathrm{~m} / \mathrm{s}$ (top) and $1593 \mathrm{~m} / \mathrm{s}$ (bottom).

Both sediment wave-speeds are of the most important parameters in this SWellEX3 bottom model. Note that if all the parameters are simultaneously mismatched by one percent, the introduced bias is about $34 \mathrm{~m}$. From Fig. 6-39, the bias given by onepercent mismatch for both wave-speeds (corresponding to the true top wave-speed at $1556 \mathrm{~m} / \mathrm{s}$ and the true bottom wave-speed at $1577 \mathrm{~m} / \mathrm{s}$ ) is about the same. The above results support our simulations in Section 6.5, in which only these two wave-speeds are included to describe the environmental uncertainty.

Finally, we should point out that the above mismatch analysis is quite efficient in computation. Only the true field and the assumed field need to be calculated. Given the environmental uncertainty, we may evaluate the effects of possible mismatch for an assumed environmental parameter set. The system mismatch, for example, with incorrect array shape parameters, can be analyzed in the same manner.

\subsection{Comparison to SWellEX-3 Data Processing}

The SWellEX-3 experiment was conducted off the coast of San Diego in 1994. Fig. 641 [17] gives the location and bathymetry of the test site. An acoustic source was 


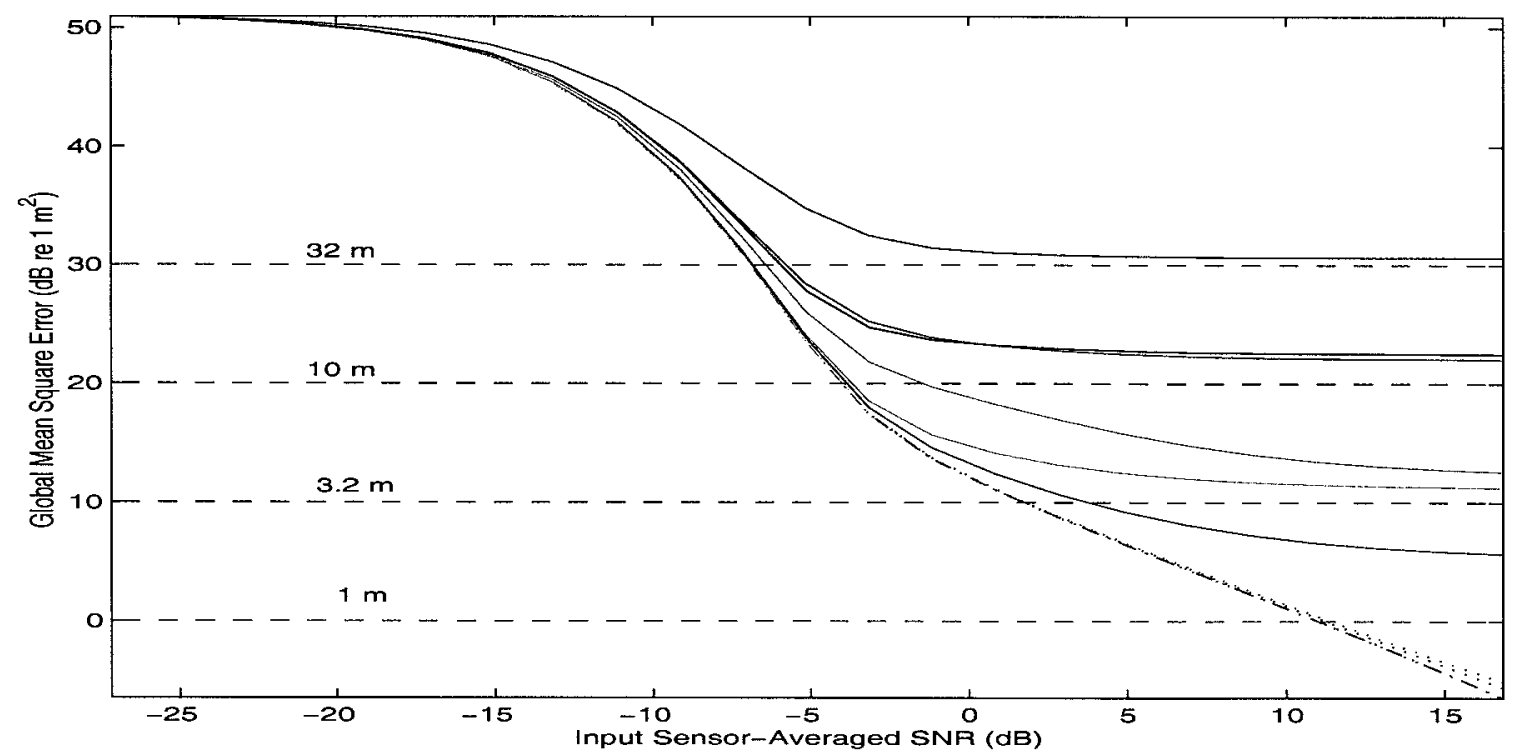

Figure 6-40: Performance bound evaluation for source range estimation with environmental mismatch. The top blue line corresponds to mismatch at all parameters; the bottom dashdot line corresponds to mismatch at no parameters. Specifications for mismatch at each individual parameter are given in Table. 6.3.

Table 6.3: True and assumed environmental models in Fig. 6-40. Except otherwise stated in Fig. 6-40, each time only one parameter is mismatched by one percent.

\begin{tabular}{|c|r||r|r||r|}
\hline \multicolumn{2}{|c|}{$\begin{array}{c}\text { Bottom } \\
\text { Property }\end{array}$} & $\begin{array}{r}\text { Assumed } \\
\text { Value }\end{array}$ & $\begin{array}{r}\text { True } \\
\text { Value }\end{array}$ & $\begin{array}{r}\text { Representation } \\
\text { in Fig. 6-40 }\end{array}$ \\
\hline \hline \multirow{3}{*}{$\begin{array}{c}\text { Sediment } \\
\text { Layer }\end{array}$} & Top Wave-Speed $(\mathrm{m} / \mathrm{s})$ & 1572 & 1556 & Red Solid \\
\cline { 2 - 5 } & Bottom Wave-Speed $(\mathrm{m} / \mathrm{s})$ & 1593 & 1577 & Black Solid \\
\cline { 2 - 5 } & Thickness $(\mathrm{m})$ & 26 & 25.74 & Cyan Solid \\
\cline { 2 - 5 } & Density $\left(\mathrm{g} / \mathrm{cm}^{3}\right)$ & 1.76 & 1.74 & Blue Dotted \\
\cline { 2 - 5 } Mudstone & Attenuation $(\mathrm{dB} / \mathrm{kmHz})$ & 0.20 & 0.198 & Red Dotted \\
\cline { 2 - 5 } Layer & Top Wave-Speed $(\mathrm{m} / \mathrm{s})$ & 1881 & 1862 & Green Solid \\
\cline { 2 - 5 } & Bottom Wave-Speed $(\mathrm{m} / \mathrm{s})$ & 3246 & 3214 & Magenta Solid \\
\cline { 2 - 5 } & Thickness $(\mathrm{m})$ & 800 & 792 & Yellow Solid \\
\cline { 2 - 5 } & Density $\left(\mathrm{g} / \mathrm{cm}^{3}\right)$ & 2.06 & 2.04 & Black Dotted \\
\cline { 2 - 5 } Basement & Attenuation $(\mathrm{dB} / \mathrm{kmHz})$ & 0.06 & 0.0594 & Green Dotted \\
\cline { 2 - 5 } & Wave-Speed $(\mathrm{m} / \mathrm{s})$ & 5200 & 5148.5 & Cyan Dotted \\
\cline { 2 - 5 } & Density $\left(\mathrm{g} / \mathrm{cm}^{3}\right)$ & 2.66 & 2.63 & Magenta Dotted \\
\cline { 2 - 5 } & Attenuation $(\mathrm{dB} / \mathrm{kmHz})$ & 0.02 & 0.0198 & Yellow Dotted \\
\hline
\end{tabular}


towed at various depths along Track $\mathrm{A}$, which was considered as a range-independent track with water depth of $198 \mathrm{~m}$. A 64-sensor vertical linear array was deployed, spanning the bottom half of the water column.

The transmitted signal included 10 pilot tones uniformly spread from $53 \mathrm{~Hz}$ to $197 \mathrm{~Hz}$. Each pilot tone was accompanied by four other low-level tones at frequencies increased by $2,4,6$, and $8 \mathrm{~Hz}$, respectively. Denote $f_{i}, i=1, \ldots, 10$, as the pilot tone frequencies. Then the input SNR's averaged across sensors and frequencies are 10, 0 , $-4,-8$, and $-12 \mathrm{~dB}$ for tone sets at $f_{i}, f_{i}+2, f_{i}+4, f_{i}+6$, and $f_{i}+8$, respectively.

On July 30 between 18:28 and 18:35 GMT, the source was held at almost a constant depth of $60 \mathrm{~m}$, and moved from $3500 \mathrm{~m}$ to about $4750 \mathrm{~m}$. Our data processing is done for this 7-minute period. To compute the tone signal, each six seconds of time series (8192 sampling points) is Fourier analyzed using a 50\% overlapped KaiserBessel window. Accordingly, there are 154 snapshots for each tone in this period. All 50 tone signals are used, which are provided by Phil Schey at SPAWAR San Diego.

16 of the original 64 sensors are used with spacing of $5.625 \mathrm{~m}$, equal to the halfwavelength at $133 \mathrm{~Hz}$. The bottom sensor is at $192 \mathrm{~m}$. To reduce the effects of source motion, only a single snapshot is used for each tone but all 10 tones at about the same level are used simultaneously. To calculate the replica field, an optimized bottom model [12] is used as shown in Fig. 6-42, which is slightly different from the model in Fig. 4-1.

Figs. 6-43 through 6-46 give the source localization results. The search interval is $3500 \mathrm{~m}$ to $4750 \mathrm{~m}$ with $10 \mathrm{~m}$ increment for source range, $50 \mathrm{~m}$ to $70 \mathrm{~m}$ with 1 $\mathrm{m}$ increment for source depth, and $8^{\circ}$ to $12^{\circ}$ with $0.5^{\circ}$ increment for array tilt. The maximum likelihood estimator in (4.25) is used to find estimates of the range, depth and array tilt. At input SNR $=10 \mathrm{~dB}$, we see that all the range estimates are around the mainlobe and follow the source track well. As SNR decreases, some estimates occur at sidelobe points. At $\mathrm{SNR}=-4 \mathrm{~dB}$, the number of sidelobe points is over one-third of the total estimates. At SNR $=-8 \mathrm{~dB}$, no useful information regarding the source location can be found.

To compare with the bound's prediction, we now fix the source depth at the opti- 


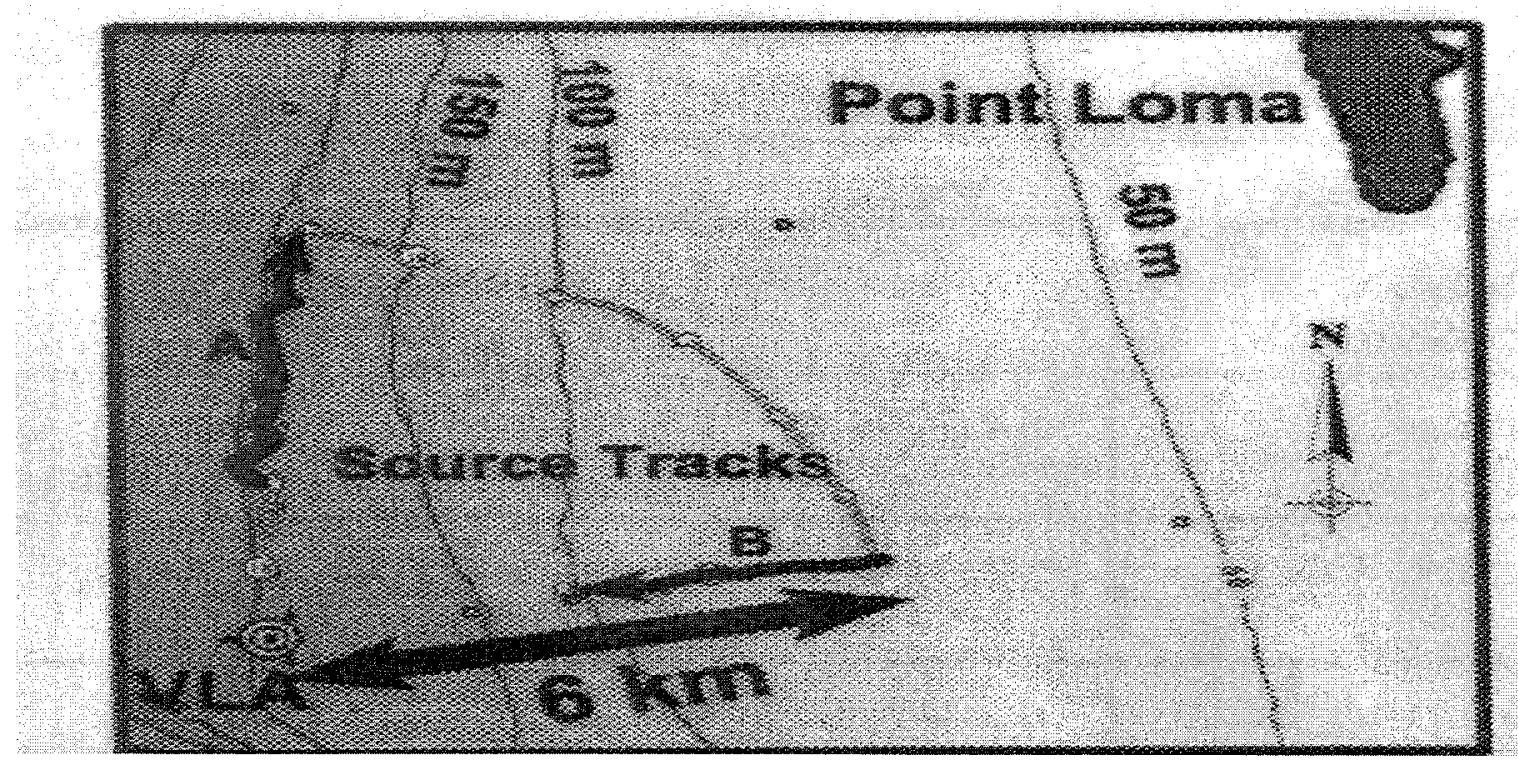

Figure 6-41: SWellEX-3 experiment area (from Ref. [17]).

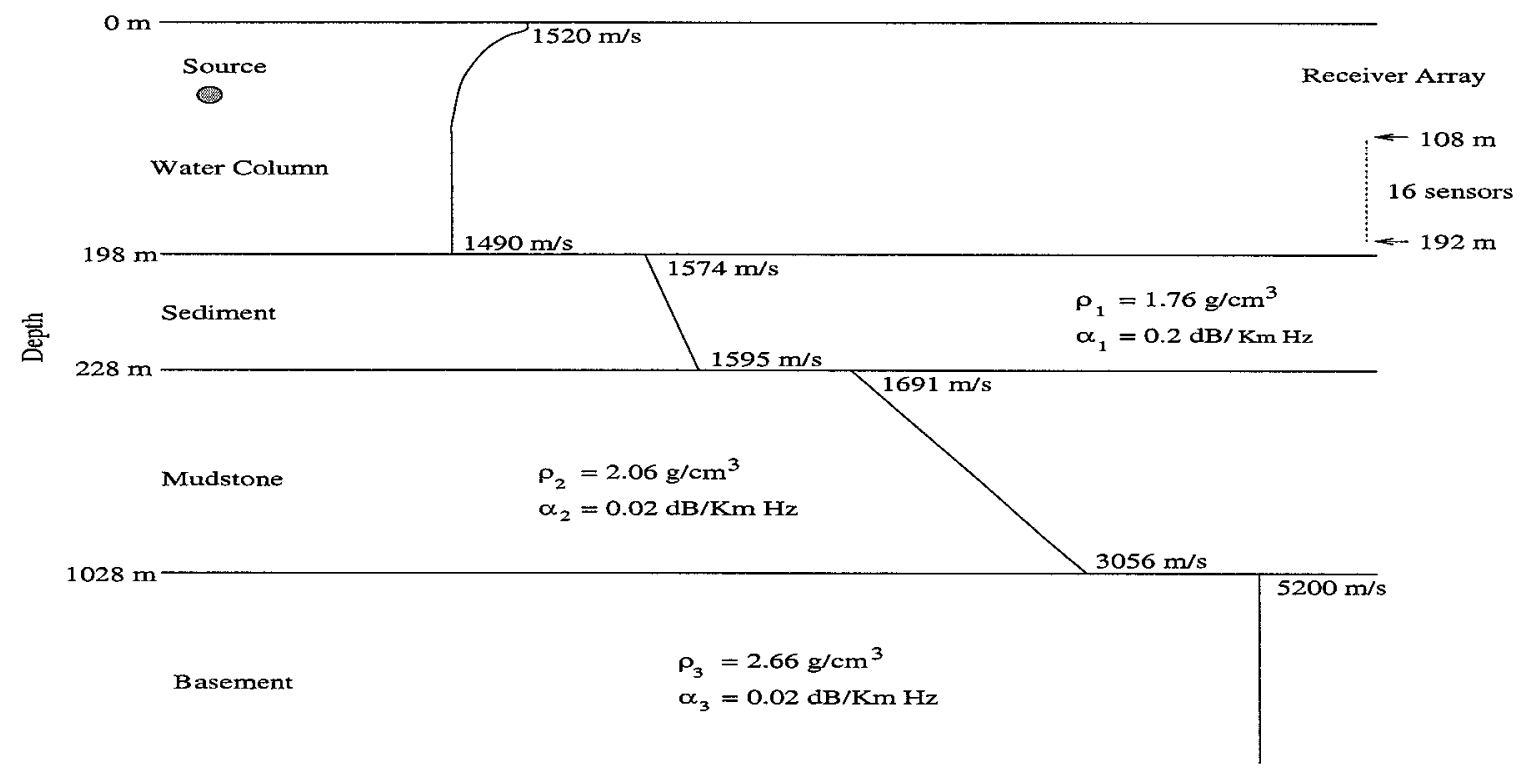

Figure 6-42: Optimized SWellEX-3 environment. Source and receiver configuration is also shown. 

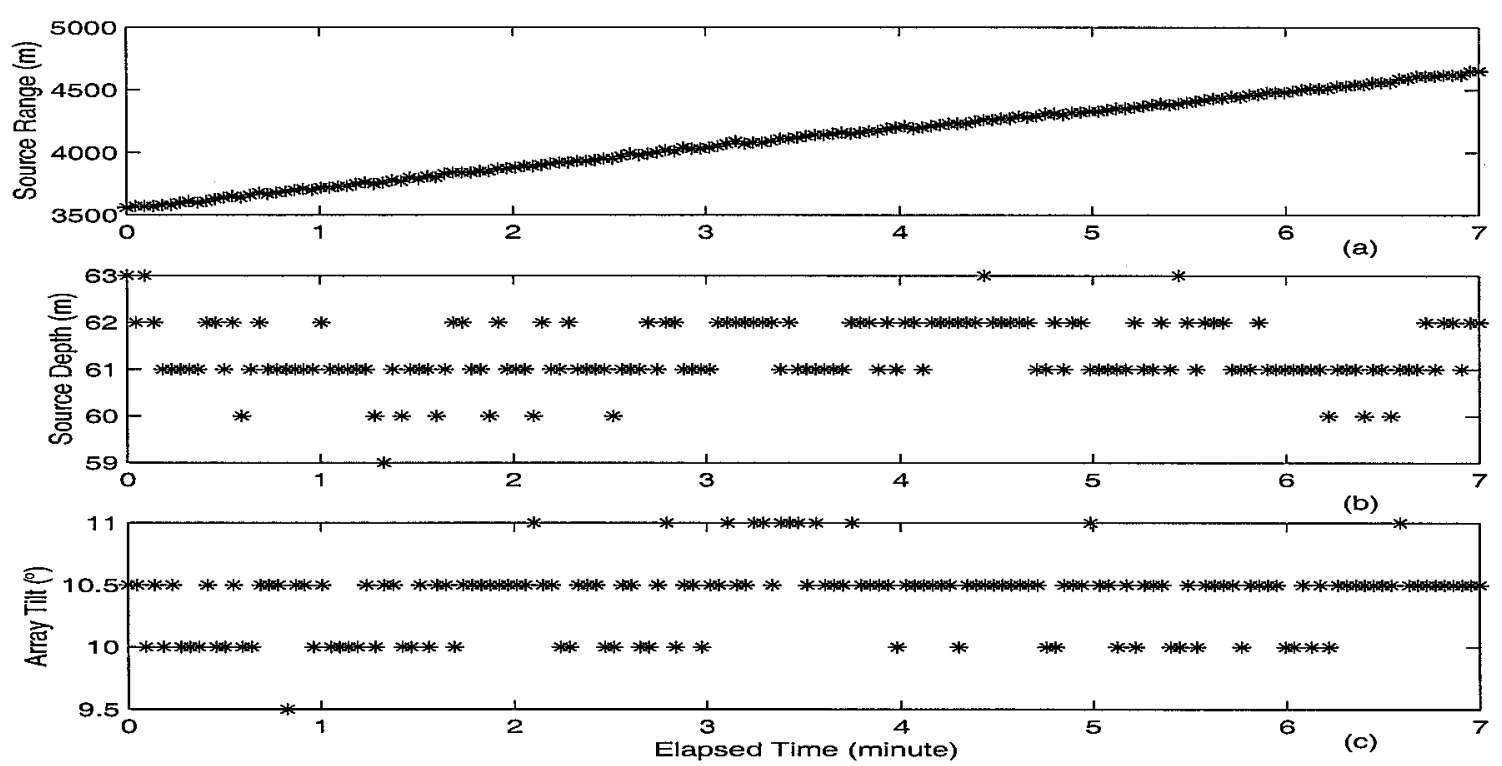

Figure 6-43: SWellEX-3 source localization: (a) source range; (b) source depth; and (c) array tilt angle. The input sensor-averaged SNR is $10 \mathrm{~dB}$.
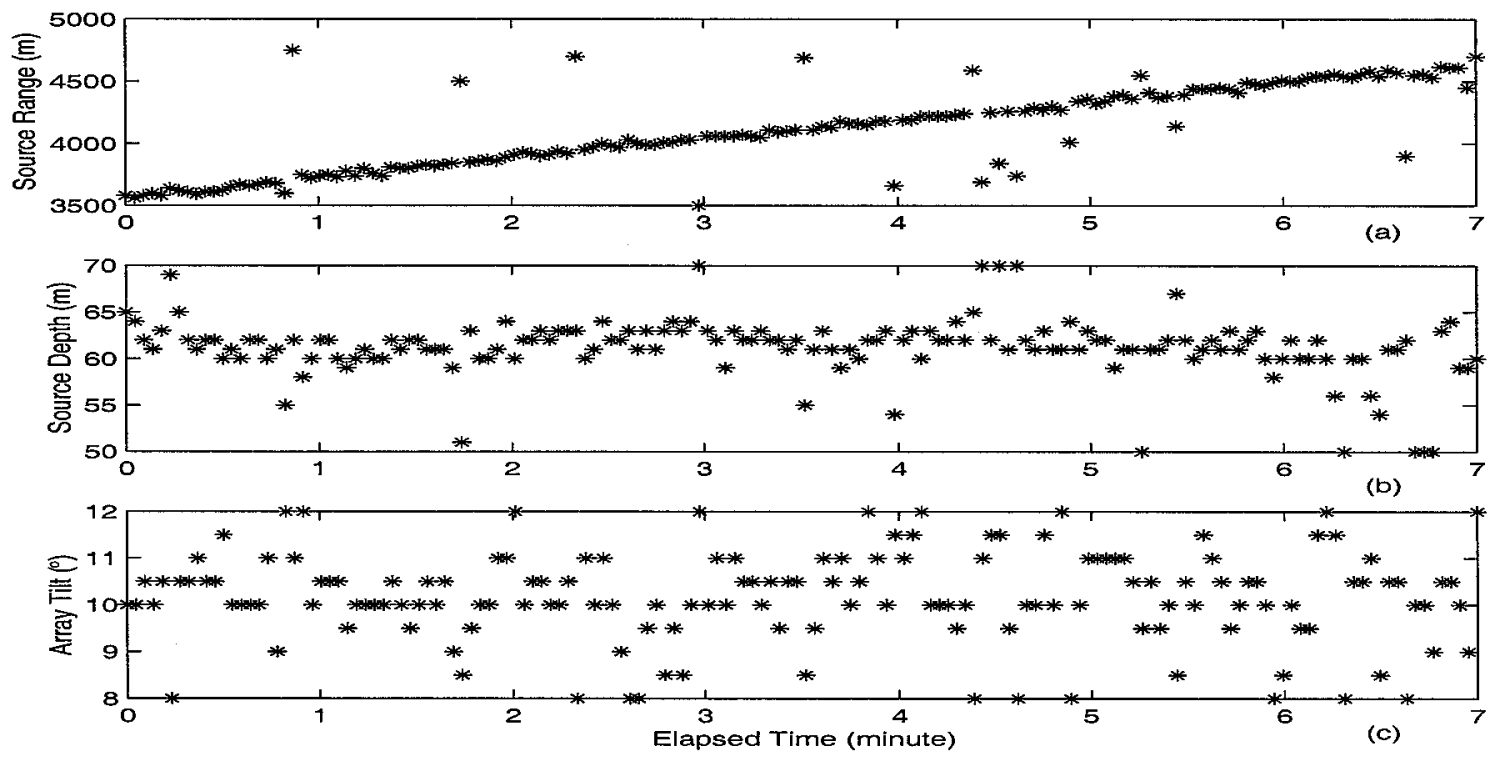

Figure 6-44: SWellEX-3 source localization: (a) source range; (b) source depth; and (c) array tilt angle. The input sensor-averaged SNR is $0 \mathrm{~dB}$. 

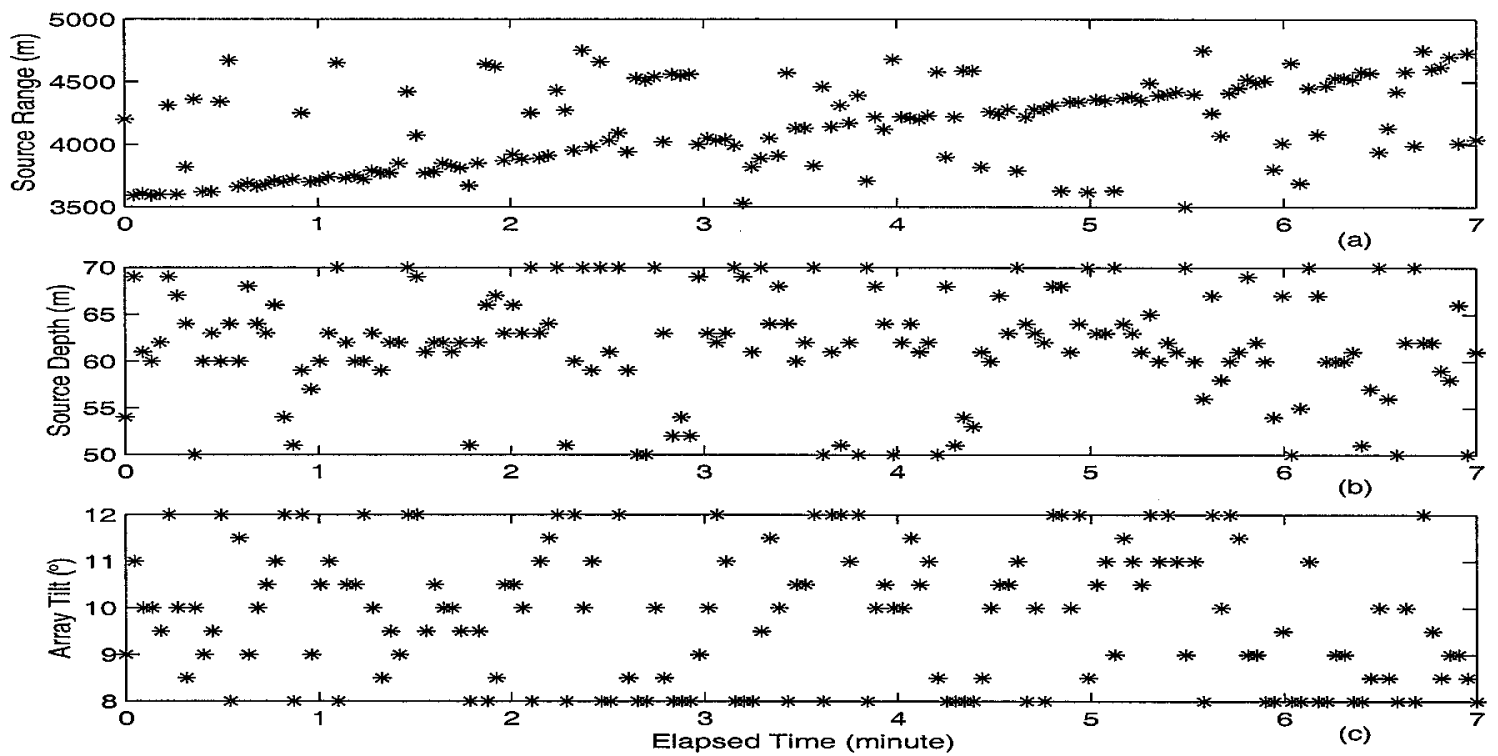

Figure 6-45: SWellEX-3 source localization: (a) source range; (b) source depth; and (c) array tilt angle. The input sensor-averaged SNR is $-4 \mathrm{~dB}$.
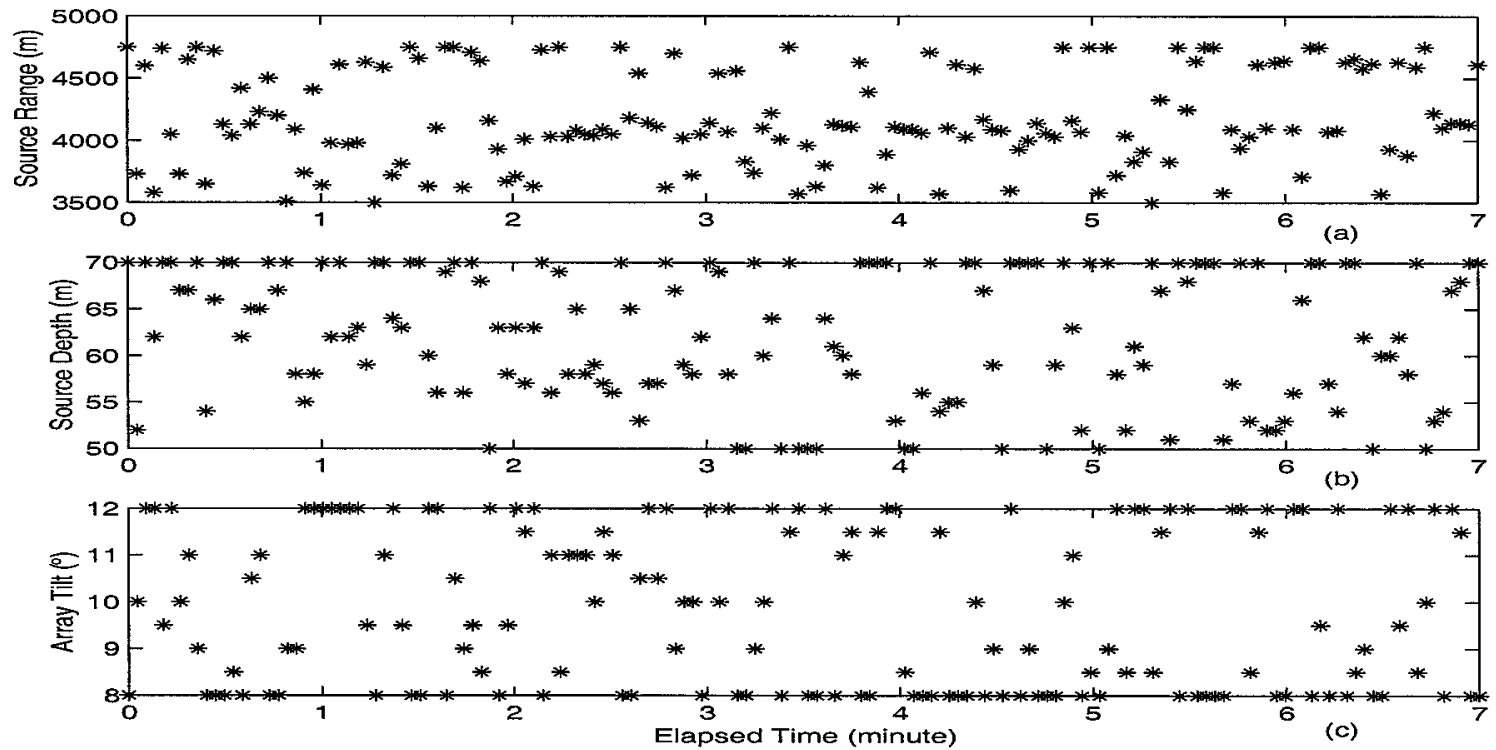

Figure 6-46: SWellEX-3 source localization: (a) source range; (b) source depth; and (c) array tilt angle. The input sensor-averaged SNR is $-8 \mathrm{~dB}$. 


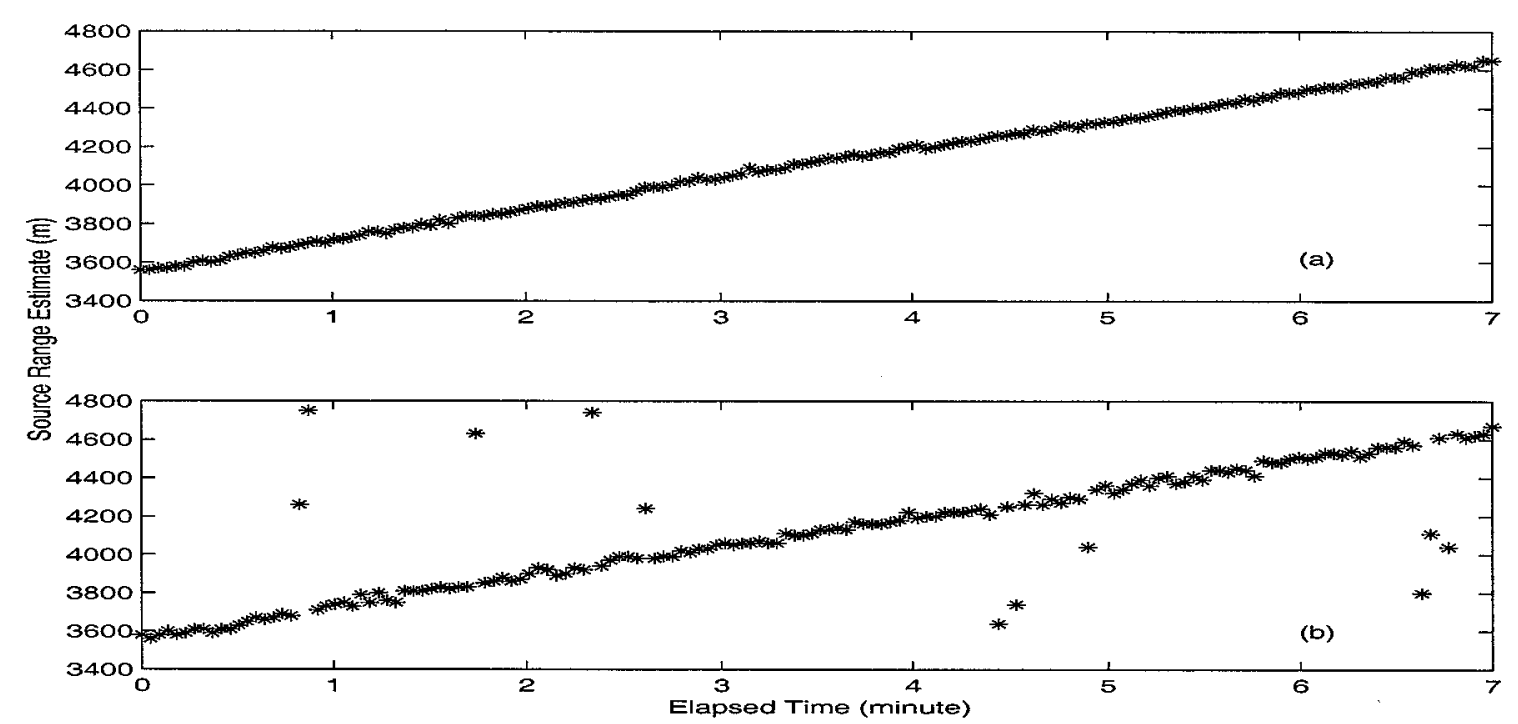

Figure 6-47: SWellEX-3 source range estimation. The input sensor-averaged SNR is (a) $10 \mathrm{~dB}$; (b) $0 \mathrm{~dB}$.

mum value of $61 \mathrm{~m}$ and array tilt at $10.5^{\circ}$, so the source range is the only parameter to be estimated. Figs. 6-47 and 6-48 present the results. Again, at SNR $=10 \mathrm{~dB}$, all the range estimates are around the mainlobe. At SNR $=0 \mathrm{~dB}$, there are 11 estimates at sidelobe points. At SNR $=-4 \mathrm{~dB}$, the number of sidelobe points is 36 , about $25 \%$ of the total estimates. We define this SNR as the threshold SNR. At $\mathrm{SNR}=-8 \mathrm{~dB}$, we can still find some information about the source track although the sidelobe points dominate the estimation. Compared to Fig. 6-49(a), which gives the Ziv-Zakai bound for the same configuration, the threshold SNR is about the same $(-4 \mathrm{~dB}$ versus $-5 \mathrm{~dB})$. The two-point error analysis also predicts a threshold SNR at $-5 \mathrm{~dB}$, as shown in Fig. 6-50(a). For simultaneous range, depth and array tilt estimation (Fig. 6-50(b)), the predicted threshold SNR is $-3 \mathrm{~dB}, 2 \mathrm{~dB}$ higher than the single range estimation case. This also matches the data processing in Figs. 6-43 through 6-46. Note that for input SNR $=-5 \mathrm{~dB}(-3 \mathrm{~dB})$, the corresponding output SNR is $12 \mathrm{~dB}(14 \mathrm{~dB})$.

It is interesting to note that although the source track is well followed at high SNR, the ambiguity mainlobe is quite wide, in the order of tens of meters, as shown in Fig. 6-51. This suggests that a systematic bias could exist. When plotting the 


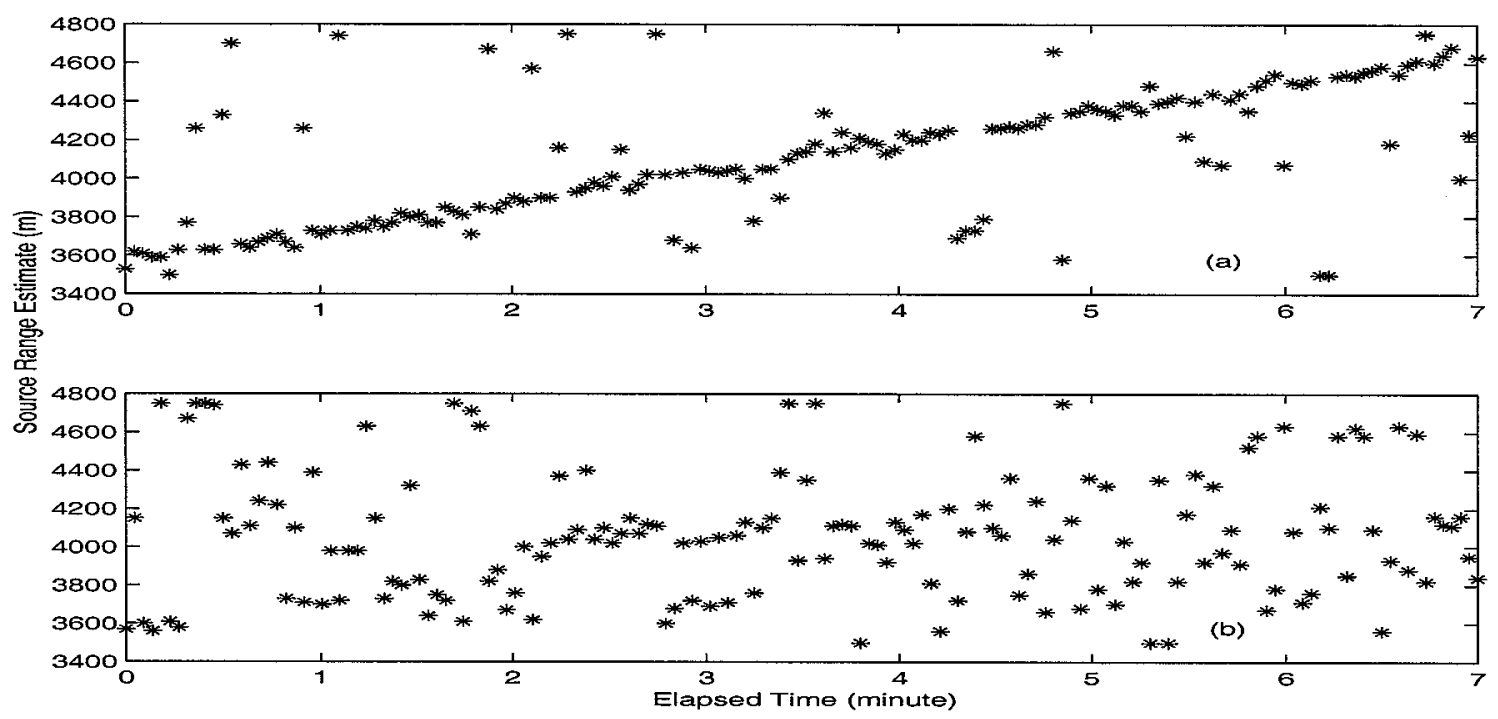

Figure 6-48: SWellEX-3 source range estimation. The input sensor-averaged SNR is (a) $-4 \mathrm{~dB}$; (b) $-8 \mathrm{~dB}$.

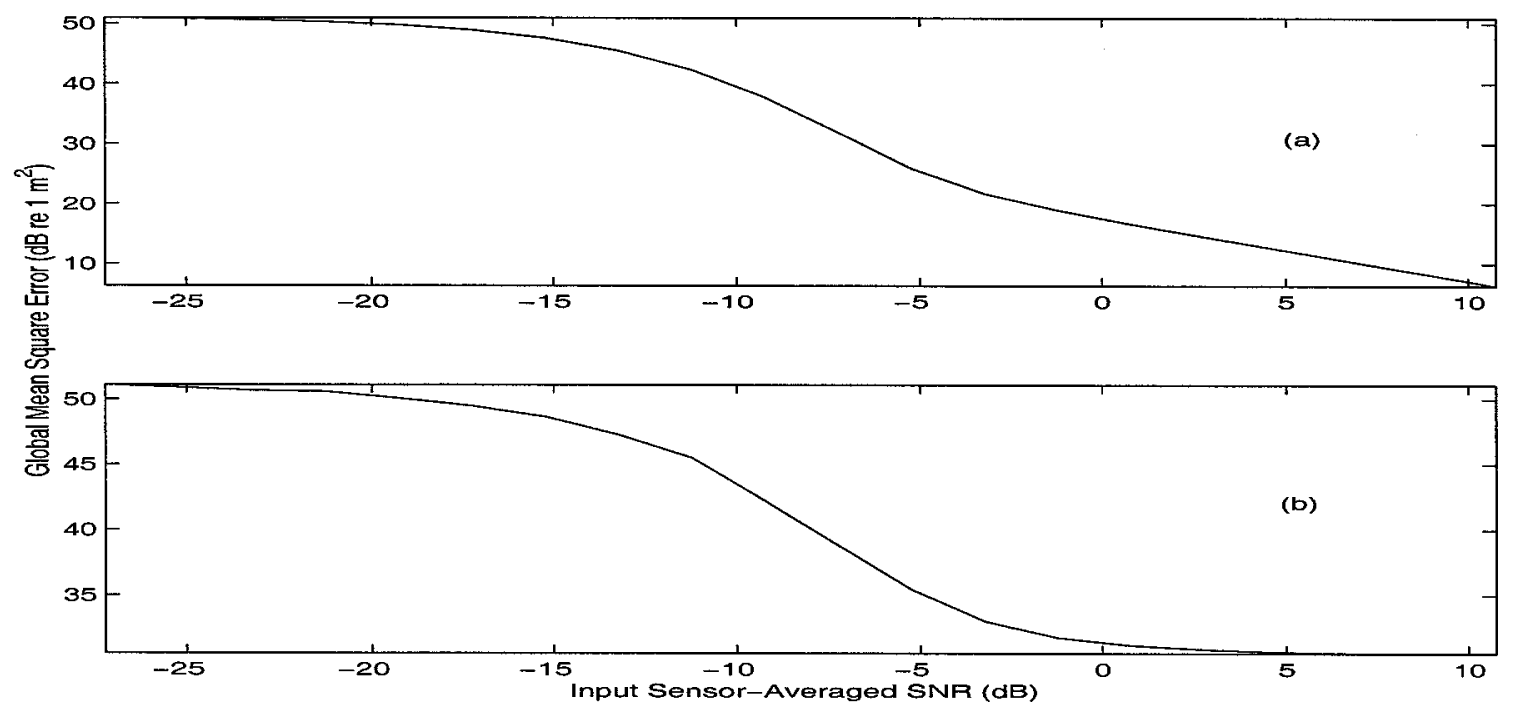

Figure 6-49: Ziv-Zakai bound evaluation for source range estimation: (a) without mismatch; (b) with $1 \mathrm{~dB}$ correlation loss caused by mismatch. 

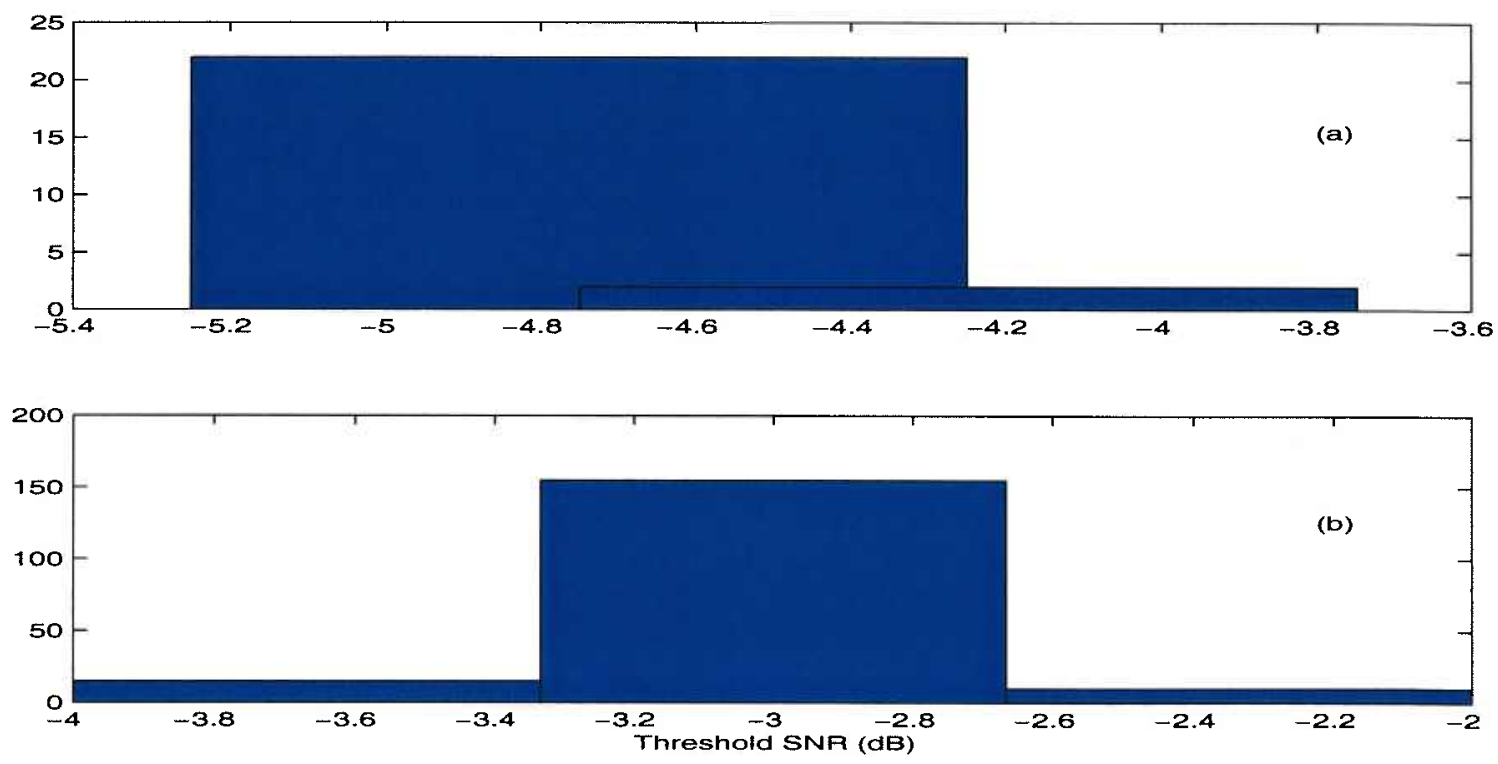

Figure 6-50: Histogram of threshold SNR for source range estimation predicted at individual source/array locations: (a) known source depth and array tilt; (b) unknown source depth and array tilt.

MFP estimates together with the DGPS measurements (Fig. 6-52), we can clearly see some offsets between them. The averaged offset is about $80 \mathrm{~m}$. Some possible factors contributing to the offset include the DGPS accuracy, offset from the DGPS station to the towed source, offset from the DGPS station to the receiver array, and source motion. These could account for $30 \mathrm{~m}$ to $50 \mathrm{~m}$ offset. Another factor is the mismatch-introduced bias. Indeed, there is about one $\mathrm{dB}$ correlation loss due to mismatch as observed in Fig. 6 of Ref. [17]. Given one dB correlation loss, we evaluate the MZZB and the predicted bias is about $37 \mathrm{~m}$ (cf. Fig. 6-49(b)).

Overall, the performance predictions give quite good indications of the threshold SNR as well as the mismatch-introduced bias in SWellEX-3 data processing.

\subsection{Summary and Discussions}

In this chapter, three Bayesian performance bounds are tested for some typical matched-field problems. All three bounds try to capture the ambiguity in parameter estimation but in different ways and thus predict the performance with different tightness. Overall, for most intermediate to high SNR levels, the Ziv-Zakai bound 


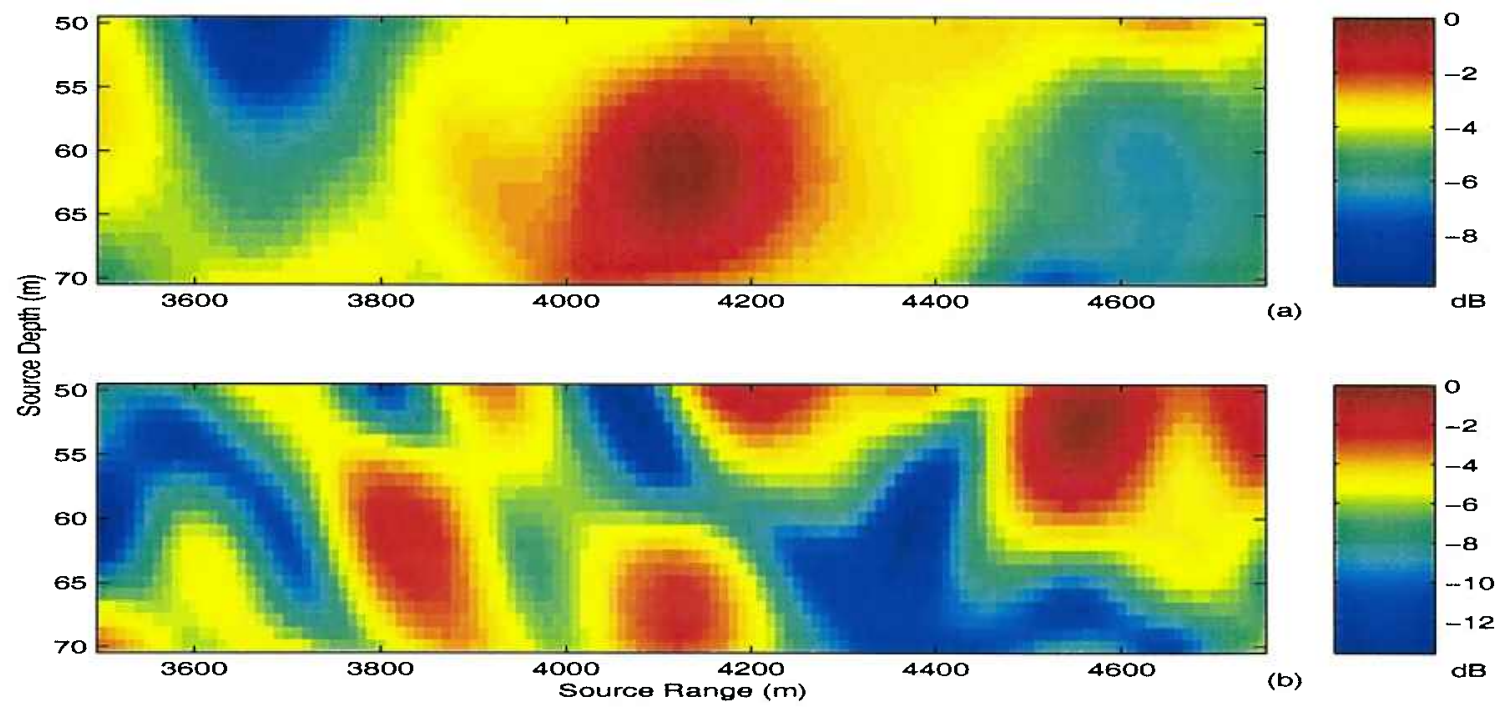

Figure 6-51: Example ambiguity surface: (a) $\mathrm{SNR}=10 \mathrm{~dB}$, peak at the mainlobe; (b) $\mathrm{SNR}=-4 \mathrm{~dB}$, peak at the sidelobe.

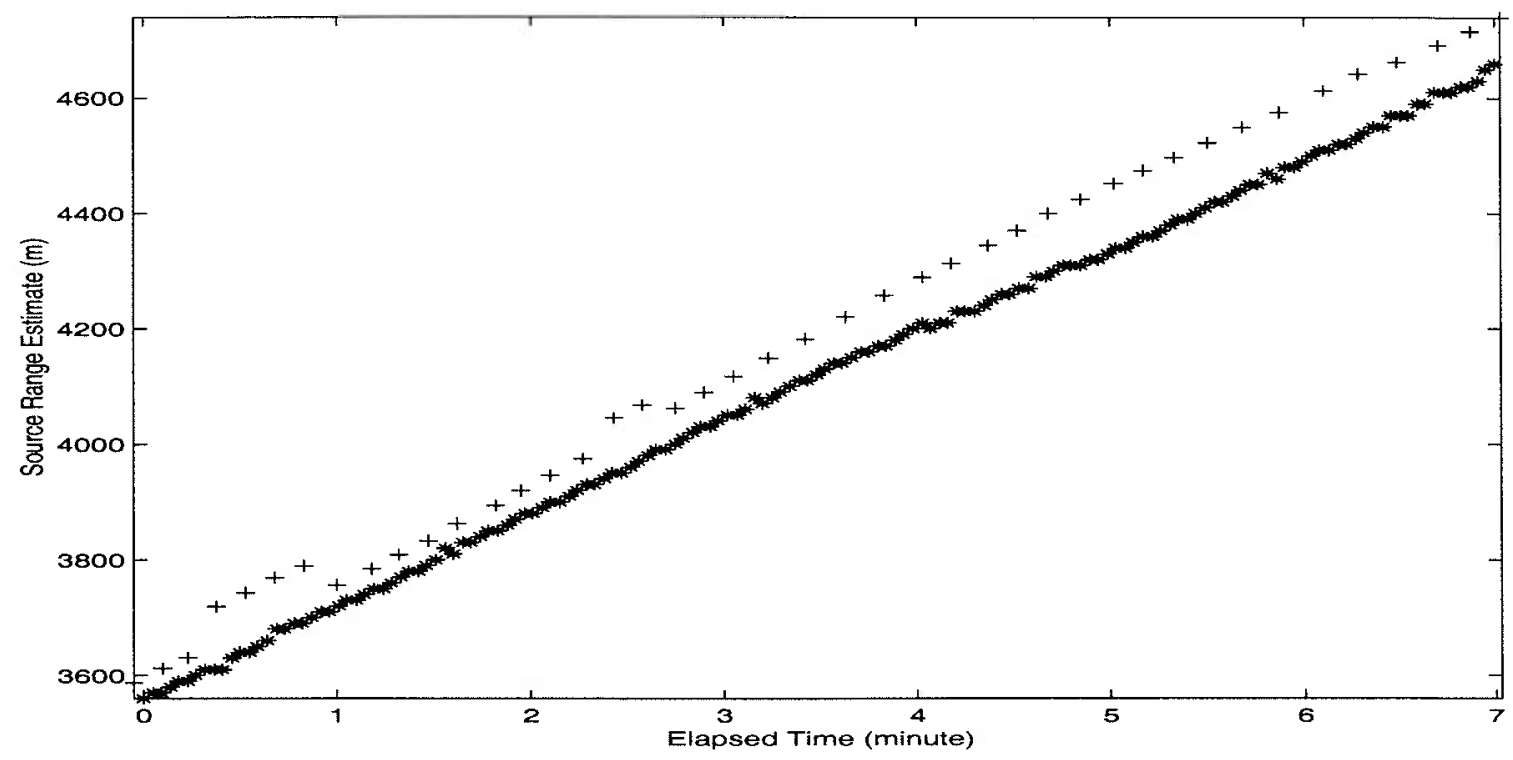

Figure 6-52: SWellEX-3 source range estimation by DGPS $(+)$ and MLE $\left(^{*}\right)$. The input sensor-averaged SNR for MLE is $10 \mathrm{~dB}$. 
gives the best performance predictions.

For the scalar parameter case, the ZZB is applied to determine the necessary number of independent snapshots to achieve the asymptotic performance at a given SNR. It is also used to evaluate a popular broadband (multi-tone) processing approach, incoherent frequency averaging, which reduces the sidelobe level, but may not dramatically improve the overall performance due to the increased ambiguity baseline. For the vector parameter case, we have observed an increased threshold SNR but similar asymptotic performance by adding a weakly-coupled parameter, or higher asymptotic mean square errors by adding a strongly-coupled parameter. Finally, the proposed modified ZZB is tested under different mismatch scenarios.

Parallel to the development of the performance bound, error analyses using the two-point estimation error probability have been developed. This approach is used to explain different ambiguity phenomena and thus estimate the threshold SNR in MLE simulations. The error analysis results can be summarized by a plot of probabilistic square error at each scanning parameter point as a function of SNR and a contour plot of threshold SNR predicted within the parameter space. These, combined with the parameter coupling plot given by the local Cramer-Rao bound and the contour plot of bias predicted by the modified ZZB for given mismatches, provide comprehensive images of the field properties.

One may notice that a slight mismatch could degrade the performance significantly, as shown in Figs. 6-38 and 6-40. Indeed, some robust algorithms have been developed. For example, some of the approaches use only a subset of the propagation modes, or a subspace of that spanned by the propagation modes. This reduces the field sensitivity to some environmental parameter perturbations. Accordingly, we can evaluate the bound with the signal field pre-processed in the same way.

The bound's predictions agree well with the real data processing under optimized conditions. This demonstrates the current modeling, including environmental modeling, sound propagation modeling and statistical modeling, has developed to such a level that the optimum theoretical performance can be achieved in a well-controlled experiment. 


\section{Chapter 7}

\section{Concluding Remarks}

\subsection{Conclusion}

In this thesis, the Bayesian Cramer-Rao bound, Weiss-Weinstein bound and Ziv-Zakai bound are introduced for performance analysis in the matched-field problem. Evaluations of the WWB and ZZB are closely related to signal ambiguity function, thus making them important tools for analyzing the threshold phenomenon in matchedfield parameter estimation. It is shown that the Bayesian CRB and WWB may not be achievable even at high SNR, depending on how local performances vary across the parameter interval. On the contrary, the Ziv-Zakai bound gives the best performance prediction for most SNR levels of practical interest.

Fundamentally, ambiguity properties determine the performance in any parameter estimation problem. Some typical ambiguity features include mainlobe and sidelobe. The sidelobe error contribution is determined by its level as well as distance from the true parameter point, while the mainlobe shape determines the mainlobe error contribution, which is large in the presence of mainlobe protrusion. The threshold SNR is determined by the relative size of the mainlobe and sidelobe contributions. All those can be quantitatively analyzed using the two-point estimation error probability.

For a source localization problem, the Bayesian performance bound naturally incorporates environmental uncertainty by assuming a random distribution model for each uncertain parameter. Adding a weakly-coupled parameter does not degrade 
the asymptotic performance but could increase the threshold SNR due to the added sidelobe ambiguity; adding a strongly-coupled parameter degrades the asymptotic performance due to the added mainlobe ambiguity but the sidelobe threshold may not be increased. This can be analyzed based on the one-dimensional projection of a multi-dimensional ambiguity function.

The mismatch problem has also been analyzed using a modified two-point estimation error probability. This is done by identifying different environmental/system parameter sets embedded in the observation and the replica. The mismatched error is determined by the level degradation and location shift of the field correlation peak and thus two mismatched parameters may behave in a coupled way. The resulting modified ZZB well describes the performance in parameter estimation under mismatched conditions. Indeed, the size of bias can be estimated by taking the square-root of the modified ZZB at high SNR.

Finally, the developed performance analyses have been verified against simulations as well as real data processing. A comprehensive description of the matched-field performance is now available by combining the results in this thesis and some previous results for asymptotic performance. This can be summarized by three plots. The first plot describes the local resolution and coupling across the parameter space in the asymptotic region, which characterizes the mainlobe behavior. The second one

gives the contour of threshold SNR predicted over the parameter space, which is a good indication of the sidelobe behavior. The third one provides a contour of bias in estimation of one parameter given a set of possible mismatches in other parameters.

\subsection{Summary of Contributions}

1. A two-point estimation error analysis approach has been developed, which makes the quantitative probability analysis of matched-field ambiguity possible.

2. Large-error Bayesian performance bounds have been developed for source localization and estimation of environmental parameters using matched-field meth- 
ods. They give tight and reliable performance predictions in all SNR regions and thus provide benchmarks to which various ad hoc algorithms can be compared.

3. A large-error performance bound is proposed for parameter estimation in the presence of environmental/system mismatch. This is the first of its type and can be a useful tool for performance analysis in practical sonar/radar applications.

4. Effects of adding additional parameter(s) are quantitatively investigated in terms of the parameter coupling. The results are important for understanding the performance in a multi-dimensional parameter estimation problem.

5. Error analysis for binary hypotheses test has been further developed from the results in Ref. [72]. This can be applied to general stochastic signal detection and estimation problems.

6. The last one, and maybe the most important one, belongs to many individuals, which is demonstrated through this author. Performance predictions are compared to experimental results and some close consistency is observed, indicating that the current theoretical modeling efforts and experimental efforts are well united.

\subsection{Future Work}

The computation required is the main concern in applying a global performance bound to more complicated multi-dimensional problem. In addition to some optimization algorithms, a better understanding and then a better model for the environmental effects would be desired.

Currently, only the effects of white noise are considered. It would be interesting to evaluate the performance under correlated noise fields or in the presence of other interferences. This can be done by adding each individual contribution to the data model. In addition, application to range-dependent environments is a natural step to follow. 
The error analysis in this thesis is developed in the context of the maximum likelihood estimate. This approach can be generalized to include other processors, for example, the minimum variance distortionless response (MVDR) processor. To do so, we need to solve the error probability in a two-point estimation problem using MVDR.

In typical matched-field experiments, the source is often in moving by itself or towed by a surface ship. To better predict the performance for such experiments, one may want to modify the statistical data model to include the effects of source motion, which requires significant extra effort but is practically very important. 


\section{Appendix A}

\section{Weiss-Weinstein Bound \\ Derivations for MFP/MFT}

Calculation of the Weiss-Weinstein bound relies upon evaluating the function $\mu(s, \mathbf{h})$. $\mu(s, \mathbf{h})$ is defined in terms of the probability model by

$$
\mu(s, \mathbf{h})=\ln E\left[\left(\frac{p(\mathbf{R}, \boldsymbol{\theta}+\mathbf{h})}{p(\mathbf{R}, \boldsymbol{\theta})}\right)^{s}\right]
$$

In this appendix we derive $\mu(s, \mathbf{h})$ using the multiple-frequency and multiplesnapshot data model in Section 3.2.1. The data model assumes zero-mean Gaussian distributions for both signal and noise terms, so only the covariance matrix matters. Based on the eigen-structure of the covariance matrix, $\mu(s, \mathbf{h})$ is further simplified for better physical understanding and efficient computation.

\section{A.1 Derivations of $\mu(s, \mathbf{h})$}

First note that

$$
\begin{aligned}
\mu(s, \mathbf{h}) & =\ln \left[\int_{\Theta} d \boldsymbol{\theta} \int_{\Omega} d \mathbf{R} p^{s}(\mathbf{R}, \boldsymbol{\theta}+\mathbf{h}) p^{1-s}(\mathbf{R}, \boldsymbol{\theta})\right] \\
& =\ln \left[\int_{\Theta} d \boldsymbol{\theta} p^{s}(\boldsymbol{\theta}+\mathbf{h}) p^{1-s}(\boldsymbol{\theta}) \int_{\Omega} d \mathbf{R} p^{s}(\mathbf{R} \mid \boldsymbol{\theta}+\mathbf{h}) p^{1-s}(\mathbf{R} \mid \boldsymbol{\theta})\right]
\end{aligned}
$$


where $p(\boldsymbol{\theta})$ is the prior pdf of the parameter set, and the conditional pdf is given by (cf. Section 3.2.1):

$$
\begin{aligned}
p(\mathbf{R} \mid \boldsymbol{\theta}) & =\frac{1}{\left|\pi \mathrm{K}_{\mathbf{R}}(\boldsymbol{\theta})\right|} \exp \left(-\mathbf{R}^{\dagger} \mathrm{K}_{\mathbf{R}}^{-1}(\boldsymbol{\theta}) \mathbf{R}\right) \\
& =\frac{1}{\prod_{m=1}^{M}\left|\pi \mathrm{K}_{\mathbf{R}}\left(f_{m}, \boldsymbol{\theta}\right)\right|^{L}} \prod_{l=1}^{L} \prod_{m=1}^{M} \exp \left(-\mathbf{R}_{l}^{\dagger}\left(f_{m}\right) \mathrm{K}_{\mathbf{R}}^{-1}\left(f_{m}, \boldsymbol{\theta}\right) \mathbf{R}_{l}\left(f_{m}\right)\right)
\end{aligned}
$$

Recall that $L$ is the number of snapshots and $M$ is the number of frequencies.

The second integral in (A.2) can then be evaluated by

$$
\begin{aligned}
& \int_{\Omega} d \mathbf{R} p^{s}(\mathbf{R} \mid \boldsymbol{\theta}+\mathbf{h}) p^{1-s}(\mathbf{R} \mid \boldsymbol{\theta}) \\
= & \int_{\Omega} d \mathbf{R}\left(\frac{1}{\left|\pi \mathrm{K}_{\mathbf{R}}(\boldsymbol{\theta}+\mathbf{h})\right|} \exp \left(-\mathbf{R}^{\dagger} \mathrm{K}_{\mathbf{R}}^{-1}(\boldsymbol{\theta}+\mathbf{h}) \mathbf{R}\right)\right)^{s} \\
& \cdot\left(\frac{1}{\left|\pi \mathrm{K}_{\mathbf{R}}(\boldsymbol{\theta})\right|} \exp \left(-\mathbf{R}^{\dagger} \mathrm{K}_{\mathbf{R}}^{-1}(\boldsymbol{\theta}) \mathbf{R}\right)\right)^{1-s} \\
= & \int_{\Omega} d \mathbf{R} \frac{1}{\left|\pi \mathrm{K}_{\mathbf{R}}(\boldsymbol{\theta}+\mathbf{h})\right|^{s}\left|\pi \mathrm{K}_{\mathbf{R}}(\boldsymbol{\theta})\right|^{1-s}} \exp \left(-\mathbf{R}^{\dagger} \mathrm{K}^{-1}(s, \boldsymbol{\theta}, \mathbf{h}) \mathbf{R}\right),
\end{aligned}
$$

where

$$
\mathrm{K}^{-1}(s, \boldsymbol{\theta}, \mathbf{h})=s \mathrm{~K}_{\mathbf{R}}^{-1}(\boldsymbol{\theta}+\mathbf{h})+(1-s) \mathrm{K}_{\mathbf{R}}^{-1}(\boldsymbol{\theta}) .
$$

$\mathrm{K}^{-1}(s, \boldsymbol{\theta}, \mathrm{h})$ is a Hermitian and positive definite matrix for $0 \leq s \leq 1$ since both $K_{\mathbf{R}}(\boldsymbol{\theta})$ and $\mathrm{K}_{\mathbf{R}}(\boldsymbol{\theta}+\mathbf{h})$ are Hermitian and positive definite matrices. This leads to

$$
\begin{aligned}
& \int_{\Omega} d \mathbf{R} p^{s}(\mathbf{R} \mid \boldsymbol{\theta}+\mathbf{h}) p^{1-s}(\mathbf{R} \mid \boldsymbol{\theta}) \\
= & \frac{|\mathrm{K}(s, \boldsymbol{\theta}, \mathbf{h})|}{\left|\mathrm{K}_{\mathbf{R}}(\boldsymbol{\theta}+\mathbf{h})\right|^{s}\left|\mathrm{~K}_{\mathbf{R}}(\boldsymbol{\theta})\right|^{1-s}} \int_{\Omega} d \mathbf{R} \frac{1}{|\pi \mathrm{K}(s, \boldsymbol{\theta}, \mathbf{h})|} \exp \left(-\mathbf{R}^{\dagger} \mathrm{K}^{-1}(s, \boldsymbol{\theta}, \mathbf{h}) \mathbf{R}\right) \\
= & \frac{|\mathrm{K}(s, \boldsymbol{\theta}, \mathbf{h})|}{\left|\mathrm{K}_{\mathbf{R}}(\boldsymbol{\theta}+\mathbf{h})\right|^{s}\left|\mathrm{~K}_{\mathbf{R}}(\boldsymbol{\theta})\right|^{1-s}} \\
= & \frac{1}{\left|\mathrm{~K}_{\mathbf{R}}(\boldsymbol{\theta}+\mathbf{h})\right|^{s}\left|\mathrm{~K}_{\mathbf{R}}(\boldsymbol{\theta})\right|^{1-s}\left|s \mathrm{~K}_{\mathbf{R}}^{-1}(\boldsymbol{\theta}+\mathbf{h})+(1-s) \mathrm{K}_{\mathbf{R}}^{-1}(\boldsymbol{\theta})\right|}
\end{aligned}
$$


Therefore, we obtain for $\mu(s, \mathbf{h})$

$$
\begin{aligned}
\mu(s, \mathbf{h}) & =\ln \left[\int_{\Theta} d \boldsymbol{\theta} \frac{p^{s}(\boldsymbol{\theta}+\mathbf{h}) p^{1-s}(\boldsymbol{\theta})}{\left|\mathrm{K}_{\mathbf{R}}(\boldsymbol{\theta}+\mathbf{h})\right|^{s}\left|\mathrm{~K}_{\mathbf{R}}(\boldsymbol{\theta})\right|^{1-s}\left|s \mathrm{~K}_{\mathbf{R}}^{-1}(\boldsymbol{\theta}+\mathbf{h})+(1-s) \mathrm{K}_{\mathbf{R}}^{-1}(\boldsymbol{\theta})\right|}\right] \\
& =\ln \left[\int_{\Theta} d \boldsymbol{\theta} \frac{p^{s}(\boldsymbol{\theta}+\mathbf{h}) p^{1-s}(\boldsymbol{\theta})}{\prod_{m=1}^{M} \eta^{L}\left(f_{m}, s, \boldsymbol{\theta}, \mathbf{h}\right)}\right]
\end{aligned}
$$

where $\eta\left(f_{m}, s, \boldsymbol{\theta}, \mathbf{h}\right)$ is defined by

$$
\begin{aligned}
& \eta\left(f_{m}, s, \boldsymbol{\theta}, \mathbf{h}\right)= \\
& \left|\mathrm{K}_{\mathbf{R}}\left(f_{m}, \boldsymbol{\theta}+\mathbf{h}\right)\right|^{s}\left|\mathrm{~K}_{\mathbf{R}}\left(f_{m}, \boldsymbol{\theta}\right)\right|^{1-s}\left|s \mathrm{~K}_{\mathbf{R}}^{-1}\left(f_{m}, \boldsymbol{\theta}+\mathbf{h}\right)+(1-s) \mathrm{K}_{\mathbf{R}}^{-1}\left(f_{m}, \boldsymbol{\theta}\right)\right| .
\end{aligned}
$$

\section{A.2 Derivations of $\eta\left(f_{m}, \boldsymbol{\theta}, \mathbf{h}\right)$}

In evaluation of the WWB, the basic component is $\eta\left(f_{m}, s, \boldsymbol{\theta}, \mathbf{h}\right)$ (cf. (A.7)). As discussed in Section 3.2.3, for the zero-mean Gaussian data model typical of the matched-field problem, we need to set $s$ to $1 / 2$. Let us denote for $s=1 / 2$

$$
\begin{aligned}
& \eta\left(f_{m}, \boldsymbol{\theta}, \mathbf{h}\right)= \\
& \left|\mathrm{K}_{\mathbf{R}}\left(f_{m}, \boldsymbol{\theta}+\mathbf{h}\right)\right|^{1 / 2}\left|\mathrm{~K}_{\mathbf{R}}\left(f_{m}, \boldsymbol{\theta}\right)\right|^{1 / 2}\left|1 / 2 \cdot\left(\mathrm{K}_{\mathbf{R}}^{-1}\left(f_{m}, \boldsymbol{\theta}+\mathbf{h}\right)+\mathrm{K}_{\mathbf{R}}^{-1}\left(f_{m}, \boldsymbol{\theta}\right)\right)\right| .
\end{aligned}
$$

Define the normalized Green's function as

$$
\mathbf{g}\left(f_{m}, \boldsymbol{\theta}\right)=\frac{\mathbf{G}\left(f_{m}, \boldsymbol{\theta}\right)}{\left\|\mathbf{G}\left(f_{m}, \boldsymbol{\theta}\right)\right\|}
$$

The covariance matrices can then be rewritten by

$$
\mathrm{K}_{\mathbf{R}}\left(f_{m}, \boldsymbol{\theta}\right)=\sigma_{b}^{2}\left(f_{m}\right)\left\|\mathbf{G}\left(f_{m}, \boldsymbol{\theta}\right)\right\|^{2} \mathbf{g}\left(f_{m}, \boldsymbol{\theta}\right) \mathbf{g}^{\dagger}\left(f_{m}, \boldsymbol{\theta}\right)+\sigma_{n}^{2}\left(f_{m}\right) \boldsymbol{I}
$$


and

$$
\mathrm{K}_{\mathbf{R}}\left(f_{m}, \boldsymbol{\theta}+\mathbf{h}\right)=\sigma_{b}^{2}\left(f_{m}\right)\left\|\mathbf{G}\left(f_{m}, \boldsymbol{\theta}+\mathbf{h}\right)\right\|^{2} \mathbf{g}\left(f_{m}, \boldsymbol{\theta}+\mathbf{h}\right) \mathbf{g}^{\dagger}\left(f_{m}, \boldsymbol{\theta}+\mathbf{h}\right)+\sigma_{n}^{2}\left(f_{m}\right) \mathrm{I}
$$

Written in the form of (A.11) and (A.12), eigenvalues of these two covariance matrices are easily solved and given by

$$
\left\{\begin{array}{c}
\sigma_{b}^{2}\left(f_{m}\right)\left\|\mathbf{G}\left(f_{m}, \boldsymbol{\theta}\right)\right\|^{2}+\sigma_{n}^{2}\left(f_{m}\right) \\
\sigma_{n}^{2}\left(f_{m}\right) \\
\cdots \\
\sigma_{n}^{2}\left(f_{m}\right)
\end{array}\right\}
$$

and

$$
\left\{\begin{array}{c}
\sigma_{b}^{2}\left(f_{m}\right)\left\|\mathbf{G}\left(f_{m}, \boldsymbol{\theta}+\mathbf{h}\right)\right\|^{2}+\sigma_{n}^{2}\left(f_{m}\right) \\
\sigma_{n}^{2}\left(f_{m}\right) \\
\cdots \\
\sigma_{n}^{2}\left(f_{m}\right)
\end{array}\right\}
$$

respectively. Product of eigenvalues gives the matrix determinant:

$$
\left|\mathrm{K}_{\mathbf{R}}\left(f_{m}, \boldsymbol{\theta}\right)\right|=\left(\sigma_{b}^{2}\left(f_{m}\right)\left\|\mathbf{G}\left(f_{m}, \boldsymbol{\theta}\right)\right\|^{2}+\sigma_{n}^{2}\left(f_{m}\right)\right) \cdot \sigma_{n}^{2(N-1)}\left(f_{m}\right)
$$

and

$$
\left|\mathbf{K}_{\mathbf{R}}\left(f_{m}, \boldsymbol{\theta}+\mathbf{h}\right)\right|=\left(\sigma_{b}^{2}\left(f_{m}\right)\left\|\mathbf{G}\left(f_{m}, \boldsymbol{\theta}+\mathbf{h}\right)\right\|^{2}+\sigma_{n}^{2}\left(f_{m}\right)\right) \cdot \sigma_{n}^{2(N-1)}\left(f_{m}\right)
$$

Now let us look at the third term in (A.9):

$$
1 / 2 \cdot\left(\mathrm{K}_{\mathbf{R}}^{-1}\left(f_{m}, \boldsymbol{\theta}+\mathbf{h}\right)+\mathrm{K}_{\mathbf{R}}^{-1}\left(f_{m}, \boldsymbol{\theta}\right)\right)
$$


Using the Woodbury identity [43], we have

$$
\mathrm{K}_{\mathbf{R}}^{-1}\left(f_{m}, \boldsymbol{\theta}\right)=\frac{1}{\sigma_{n}^{2}\left(f_{m}\right)}\left(1-\frac{\sigma_{b}^{2}\left(f_{m}\right)\left\|\mathbf{G}\left(f_{m}, \boldsymbol{\theta}\right)\right\|^{2}}{\sigma_{b}^{2}\left(f_{m}\right)\left\|\mathbf{G}\left(f_{m}, \boldsymbol{\theta}\right)\right\|^{2}+\sigma_{n}^{2}\left(f_{m}\right)} \mathbf{g}\left(f_{m}, \boldsymbol{\theta}\right) \mathbf{g}^{\dagger}\left(f_{m}, \boldsymbol{\theta}\right)\right)
$$

and

$$
\begin{aligned}
& \mathrm{K}_{\mathbf{R}}^{-1}\left(f_{m}, \boldsymbol{\theta}+\mathbf{h}\right)=\frac{1}{\sigma_{n}^{2}\left(f_{m}\right)} . \\
& \left(\mathrm{I}-\frac{\sigma_{b}^{2}\left(f_{m}\right)\left\|\mathbf{G}\left(f_{m}, \boldsymbol{\theta}+\mathbf{h}\right)\right\|^{2}}{\sigma_{b}^{2}\left(f_{m}\right)\left\|\mathbf{G}\left(f_{m}, \boldsymbol{\theta}+\mathbf{h}\right)\right\|^{2}+\sigma_{n}^{2}\left(f_{m}\right)} \mathbf{g}\left(f_{m}, \boldsymbol{\theta}+\mathbf{h}\right) \mathbf{g}^{\dagger}\left(f_{m}, \boldsymbol{\theta}+\mathbf{h}\right)\right) .
\end{aligned}
$$

Then,

$$
\begin{aligned}
& 1 / 2 \cdot\left(\mathrm{K}_{\mathbf{R}}^{-1}\left(f_{m}, \boldsymbol{\theta}+\mathbf{h}\right)+\mathrm{K}_{\mathbf{R}}^{-1}\left(f_{m}, \boldsymbol{\theta}\right)\right)= \\
& \frac{1}{\sigma_{n}^{2}\left(f_{m}\right)}\left[1-\frac{1}{2} \frac{\sigma_{b}^{2}\left(f_{m}\right)\left\|\mathbf{G}\left(f_{m}, \boldsymbol{\theta}\right)\right\|^{2}}{\sigma_{b}^{2}\left(f_{m}\right)\left\|\mathbf{G}\left(f_{m}, \boldsymbol{\theta}\right)\right\|^{2}+\sigma_{n}^{2}\left(f_{m}\right)} \mathbf{g}\left(f_{m}, \boldsymbol{\theta}\right) \mathbf{g}^{\dagger}\left(f_{m}, \boldsymbol{\theta}\right)\right. \\
& \left.-\frac{1}{2} \frac{\sigma_{b}^{2}\left(f_{m}\right)\left\|\mathbf{G}\left(f_{m}, \boldsymbol{\theta}+\mathbf{h}\right)\right\|^{2}}{\sigma_{b}^{2}\left(f_{m}\right)\left\|\mathbf{G}\left(f_{m}, \boldsymbol{\theta}+\mathbf{h}\right)\right\|^{2}+\sigma_{n}^{2}\left(f_{m}\right)} \mathbf{g}\left(f_{m}, \boldsymbol{\theta}+\mathbf{h}\right) \mathbf{g}^{\dagger}\left(f_{m}, \boldsymbol{\theta}+\mathbf{h}\right)\right] .
\end{aligned}
$$

In general, for $\mathbf{h} \neq 0, \mathbf{g}\left(f_{m}, \boldsymbol{\theta}\right)$ and $\mathbf{g}\left(f_{m}, \boldsymbol{\theta}+\mathbf{h}\right)$ are not co-linear (otherwise they are the same vector), and thus the last two terms in (A.20) expand a two-dimensional space. Hence, Eq. (A.20) has $(N-2)$ equal eigenvalues at $1 / \sigma_{n}^{2}\left(f_{m}\right)$, and for the remaining two, the corresponding eigenvectors are linear combinations of $\mathbf{g}\left(f_{m}, \boldsymbol{\theta}\right)$ and $\mathbf{g}\left(f_{m}, \boldsymbol{\theta}+\mathbf{h}\right)$. Denote

$$
\begin{gathered}
A\left(f_{m}, \boldsymbol{\theta}\right)=\frac{\sigma_{b}^{2}\left(f_{m}\right)\left\|\mathbf{G}\left(f_{m}, \boldsymbol{\theta}\right)\right\|^{2}}{\sigma_{b}^{2}\left(f_{m}\right)\left\|\mathbf{G}\left(f_{m}, \boldsymbol{\theta}\right)\right\|^{2}+\sigma_{n}^{2}\left(f_{m}\right)}, \\
B\left(f_{m}, \boldsymbol{\theta}, \mathbf{h}\right)=\frac{\sigma_{b}^{2}\left(f_{m}\right)\left\|\mathbf{G}\left(f_{m}, \boldsymbol{\theta}+\mathbf{h}\right)\right\|^{2}}{\sigma_{b}^{2}\left(f_{m}\right)\left\|\mathbf{G}\left(f_{m}, \boldsymbol{\theta}+\mathbf{h}\right)\right\|^{2}+\sigma_{n}^{2}\left(f_{m}\right)},
\end{gathered}
$$


and

$$
C\left(f_{m}, \boldsymbol{\theta}, \mathbf{h}\right)=\mathbf{g}^{\dagger}\left(f_{m}, \boldsymbol{\theta}\right) \mathbf{g}\left(f_{m}, \boldsymbol{\theta}+\mathbf{h}\right)
$$

We must have for those two eigenvalues ${ }^{1}$

$$
\begin{aligned}
& \left(\mathrm{I}-\frac{1}{2} A \mathbf{g}\left(f_{m}, \boldsymbol{\theta}\right) \mathbf{g}^{\dagger}\left(f_{m}, \boldsymbol{\theta}\right)-\frac{1}{2} B \mathbf{g}\left(f_{m}, \boldsymbol{\theta}+\mathbf{h}\right) \mathbf{g}^{\dagger}\left(f_{m}, \boldsymbol{\theta}+\mathbf{h}\right)\right) \\
& \cdot\left(\mathbf{g}\left(f_{m}, \boldsymbol{\theta}\right)+a \mathbf{g}\left(f_{m}, \boldsymbol{\theta}+\mathbf{h}\right)\right)=\lambda \cdot\left(\mathbf{g}\left(f_{m}, \boldsymbol{\theta}\right)+a \mathbf{g}\left(f_{m}, \boldsymbol{\theta}+\mathbf{h}\right)\right),
\end{aligned}
$$

which reduces to

$$
\begin{aligned}
& \mathbf{g}\left(f_{m}, \boldsymbol{\theta}\right) \cdot\left[1-\lambda-\frac{1}{2} A-a \frac{1}{2} A C\right]+ \\
& \mathbf{g}\left(f_{m}, \boldsymbol{\theta}+\mathbf{h}\right) \cdot\left[\left(1-\lambda-\frac{1}{2} B\right) a-\frac{1}{2} B C^{*}\right] \\
& =0 .
\end{aligned}
$$

The coefficients of $\mathbf{g}\left(f_{m}, \boldsymbol{\theta}\right)$ and $\mathbf{g}\left(f_{m}, \boldsymbol{\theta}+\mathbf{h}\right)$ must both be zero. Setting the coefficient of $\mathbf{g}\left(f_{m}, \boldsymbol{\theta}\right)$ to zero yields

$$
a=\frac{1-\lambda-\frac{1}{2} A}{\frac{1}{2} A C} \text {. }
$$

Substituting this result into the coefficient of $\mathbf{g}\left(f_{m}, \boldsymbol{\theta}+\mathbf{h}\right)$ leads to a quadratic equation for $1-\lambda$ :

$$
(1-\lambda)^{2}-\frac{A+B}{2}(1-\lambda)+\frac{1}{4} A B\left(1-|C|^{2}\right)=0
$$

Solving this equation gives the solutions for eigenvalues:

$$
\lambda=1-\frac{A+B}{4} \pm \frac{\sqrt{(A-B)^{2}+4 A B|C|^{2}}}{4}
$$

\footnotetext{
${ }^{1}$ Eigenvectors are determined up to a scalar, so only one coefficient, $a$, is sufficient to describe the linear combination.
} 
With all the eigenvalues available, we have

$$
\begin{aligned}
& \left|1 / 2 \cdot\left(\mathrm{K}_{\mathbf{R}}^{-1}\left(f_{m}, \boldsymbol{\theta}+\mathbf{h}\right)+\mathrm{K}_{\mathbf{R}}^{-1}\left(f_{m}, \boldsymbol{\theta}\right)\right)\right|=\frac{1}{\sigma_{n}^{2 N}} \\
& \cdot\left(1-\frac{A+B}{4}+\frac{\sqrt{(A-B)^{2}+4 A B|C|^{2}}}{4}\right) \\
& \cdot\left(1-\frac{A+B}{4}-\frac{\sqrt{(A-B)^{2}+4 A B|C|^{2}}}{4}\right) .
\end{aligned}
$$

Expressing (A.15) and (A.16) using the same notations in (A.21,A.22,A.23), and then combining them with (A.29), we finally obtain

$$
\begin{aligned}
\eta\left(f_{m}, \boldsymbol{\theta}, \mathbf{h}\right)= & \frac{1}{\sqrt{(1-A)(1-B)}} \\
& \cdot\left(1-\frac{A+B}{4}+\frac{\sqrt{(A-B)^{2}+4 A B|C|^{2}}}{4}\right) \\
& \cdot\left(1-\frac{A+B}{4}-\frac{\sqrt{(A-B)^{2}+4 A B|C|^{2}}}{4}\right) \\
= & \frac{1}{\sqrt{(1-A)(1-B)}} \cdot\left(1-\frac{A+B}{2}+\frac{A B}{4}\left(1-|C|^{2}\right)\right) .
\end{aligned}
$$

\section{A.3 Some Properties of $\eta\left(f_{m}, \boldsymbol{\theta}, \mathbf{h}\right)$}

\section{A.3.1 Proof of $\eta\left(f_{m}, \boldsymbol{\theta}, \mathbf{h}\right) \geq 1$ and Conditions for Equality}

We first notice that $|C|^{2} \leq 1$; thus

$$
\eta\left(f_{m}, \boldsymbol{\theta}, \mathbf{h}\right) \geq \frac{1}{\sqrt{(1-A)(1-B)}}\left(1-\frac{A+B}{2}\right),
$$

where equality holds if and only if $|C|^{2}=1$ (Some trivial cases, $A=0$ and/or $B=0$, are ignored, which correspond to the cases with noise only).

After some obvious algebra, we have

$$
\frac{1}{\sqrt{(1-A)(1-B)}}\left(1-\frac{A+B}{2}\right) \geq 1
$$


with equality if and only if $A=B$ (excluding the singular case with $A=B=1$, which correspond to the case with signal only).

(A.31) and (A.32) complete the proof of $\eta\left(f_{m}, \boldsymbol{\theta}, \mathbf{h}\right) \geq 1$, and equality holds if and only if $|C|^{2}=1$ and $A=B$.

\section{A.3.2 Approximations for $\eta\left(f_{m}, \boldsymbol{\theta}, \mathbf{h}\right)$}

1) $|C| \approx 1$

$$
\eta\left(f_{m}, \boldsymbol{\theta}, \mathbf{h}\right) \approx \frac{1}{\sqrt{(1-A)(1-B)}} \cdot\left(1-\frac{A}{2}-\frac{B}{2}\right) .
$$

For $\left\|\mathbf{G}\left(f_{m}, \boldsymbol{\theta}\right)\right\|^{2} \approx\left\|\mathbf{G}\left(f_{m}, \boldsymbol{\theta}+\mathbf{h}\right)\right\|^{2}$, we have $A \approx B$, and thus $\eta\left(f_{m}, \boldsymbol{\theta}, \mathbf{h}\right) \approx 1$. In this case, only one eigenvalue of (A.17) is distinct from the others.

2) $|C| \approx 0$

$$
\eta\left(f_{m}, \boldsymbol{\theta}, \mathbf{h}\right) \approx \frac{1}{\sqrt{(1-A)(1-B)}} \cdot\left(1-\frac{A}{2}\right)\left(1-\frac{B}{2}\right) .
$$

At high SNR levels, we have $A \approx B \approx 1$ and thus

$$
\eta\left(f_{m}, \boldsymbol{\theta}, \mathbf{h}\right) \approx 1+\frac{A^{2}}{4} \cdot \frac{1}{1-A} \gg 1
$$

3) $A \approx B$ (low noise level or weak parameter-dependence of the norm of the Green's function)

$$
\eta\left(f_{m}, \boldsymbol{\theta}, \mathbf{h}\right) \approx \frac{1}{1-A} \cdot\left(\left(1-\frac{A}{2}\right)^{2}-\frac{A^{2} \cdot|C|^{2}}{4}\right)=1+\frac{1}{4} \cdot \frac{A^{2}}{1-A} \cdot\left(1-|C|^{2}\right)
$$

When $A \approx 1, \eta\left(f_{m}, \boldsymbol{\theta}, \mathbf{h}\right)$ is extremely sensitive to the variation of $|C|$. Specifically, a small departure from $|C|=1$ could result in a significant large $\eta$. In this case, two (different) eigenvalues of (A.17) are distinct from the others. 


\section{A.3.3 A Similar Result for the Minimum Variance Distor- tionless Processor (MVDR)}

Consider the single-frequency case. The MVDR output is given by [9]:

$$
S_{M V D R}(\hat{\boldsymbol{\theta}})=\left[\mathbf{g}^{\dagger}(\hat{\boldsymbol{\theta}}) \mathbf{K}_{\mathbf{R}}\left(\boldsymbol{\theta}_{T}\right) \mathbf{g}(\hat{\boldsymbol{\theta}})\right]^{-1}
$$

where $\hat{\boldsymbol{\theta}}$ is the estimated (scanning) parameter and $\boldsymbol{\theta}_{T}$ is the true parameter.

Following a similar procedure in Appendix A.2, we obtain

$$
\begin{aligned}
S_{M V D R}(\hat{\boldsymbol{\theta}}) & =\frac{1}{\frac{1}{\sigma_{n}^{2}} \cdot\left(1-\frac{\sigma_{b}^{2}\left\|\mathbf{G}\left(\boldsymbol{\theta}_{T}\right)\right\|^{2}}{\sigma_{b}^{2}\left\|\mathbf{G}\left(\boldsymbol{\theta}_{T}\right)\right\|^{2}+\sigma_{n}^{2}}|C|^{2}\right)} \\
& =\sigma_{n}^{2} \cdot \frac{1+\frac{\sigma_{b}^{2}\left\|\mathbf{G}\left(\boldsymbol{\theta}_{T}\right)\right\|^{2}}{\sigma_{n}^{2}}}{1+\frac{\sigma_{b}^{2}\left\|\mathbf{G}\left(\boldsymbol{\theta}_{T}\right)\right\|^{2}}{\sigma_{n}^{2}}\left(1-|C|^{2}\right)},
\end{aligned}
$$

where $C=\mathbf{g}^{\dagger}\left(\boldsymbol{\theta}_{T}\right) \mathbf{g}(\hat{\boldsymbol{\theta}})$.

Eq. (A.37) is a special form of (20) in Ref. [9] for the case of white background noise. As discussed in Ref. [9], this "estimate-subtract" structure from the $1-|C|^{2}$ term in (A.38) leads to sidelobe cancellation. Specifically, Eq. (A.38) is very sensitive to the variation of $|C|$ at high SNR, and thus $|C|$ must be very close to unity for the ambiguity outputs $\left(S_{M V D R}(\hat{\boldsymbol{\theta}})\right)$ to exceed a specified level. 


\section{Appendix B}

\section{Maximum Likelihood Estimator of Source/Environmental Parameters}

Consider the data model in Section 3.2.1. The received signal vector snapshots at different frequency bins are aligned to form a column observation vector, $\mathbf{R}$. The vector length is $N \times M \times L$ with $N, M$ and $L$ denoting the numbers of sensors, frequencies and snapshots, respectively. Given the source/environmental parameter set, $\boldsymbol{\theta}$, the conditional pdf of $\mathbf{R}$ is

$$
p(\mathbf{R} \mid \boldsymbol{\theta})=\frac{1}{\prod_{m=1}^{M}\left|\pi \mathrm{K}_{\mathbf{R}}\left(f_{m}, \boldsymbol{\theta}\right)\right|^{L}} \prod_{m=1}^{M} \prod_{l=1}^{L} \exp \left(-\mathbf{R}_{l}^{\dagger}\left(f_{m}\right) \mathrm{K}_{\mathbf{R}}^{-1}\left(f_{m}, \boldsymbol{\theta}\right) \mathbf{R}_{l}\left(f_{m}\right)\right) .
$$

The maximum likelihood estimator (MLE) $[43,72]$ is obtained by maximizing the logarithm of (B.1) with respect to $\boldsymbol{\theta}$. Recall that

$$
\left|\mathrm{K}_{\mathbf{R}}\left(f_{m}, \boldsymbol{\theta}\right)\right|=\left(\sigma_{b}^{2}\left(f_{m}\right)\left\|\mathbf{G}\left(f_{m}, \boldsymbol{\theta}\right)\right\|^{2}+\sigma_{n}^{2}\left(f_{m}\right)\right) \cdot \sigma_{n}^{2(N-1)}\left(f_{m}\right),
$$

and

$$
\mathrm{K}_{\mathbf{R}}^{-1}\left(f_{m}, \boldsymbol{\theta}\right)=\frac{1}{\sigma_{n}^{2}\left(f_{m}\right)}\left(1-\frac{\sigma_{b}^{2}\left(f_{m}\right)\left\|\mathbf{G}\left(f_{m}, \boldsymbol{\theta}\right)\right\|^{2}}{\sigma_{b}^{2}\left(f_{m}\right)\left\|\mathbf{G}\left(f_{m}, \boldsymbol{\theta}\right)\right\|^{2}+\sigma_{n}^{2}\left(f_{m}\right)} \mathbf{g}\left(f_{m}, \boldsymbol{\theta}\right) \mathbf{g}^{\dagger}\left(f_{m}, \boldsymbol{\theta}\right)\right),
$$


where $\mathbf{g}\left(f_{m}, \boldsymbol{\theta}\right)$ is the normalized Green's function defined in (A.10). The log likelihood function is then given by

$$
\begin{aligned}
\ln p(\mathbf{R} \mid \boldsymbol{\theta})= & -N M L \ln \pi-L \sum_{m=1}^{M} \ln \left(\sigma_{b}^{2}\left(f_{m}\right)\left\|\mathbf{G}\left(f_{m}, \boldsymbol{\theta}\right)\right\|^{2}+\sigma_{n}^{2}\left(f_{m}\right)\right) \\
& -(N-1) L \sum_{m=1}^{M} \ln \sigma_{n}^{2}\left(f_{m}\right)-\sum_{m=1}^{M} \sum_{l=1}^{L} \mathbf{R}_{l}^{\dagger}\left(f_{m}\right) \frac{1}{\sigma_{n}^{2}\left(f_{m}\right)} \\
& \left(1-\frac{\sigma_{b}^{2}\left(f_{m}\right)\left\|\mathbf{G}\left(f_{m}, \boldsymbol{\theta}\right)\right\|^{2}}{\sigma_{b}^{2}\left(f_{m}\right)\left\|\mathbf{G}\left(f_{m}, \boldsymbol{\theta}\right)\right\|^{2}+\sigma_{n}^{2}\left(f_{m}\right)} \mathbf{g}\left(f_{m}, \boldsymbol{\theta}\right) \mathbf{g}^{\dagger}\left(f_{m}, \boldsymbol{\theta}\right)\right) \mathbf{R}_{l}\left(f_{m}\right)
\end{aligned}
$$

We assume known $\sigma_{b}^{2}\left(f_{m}\right)$ and $\sigma_{n}^{2}\left(f_{m}\right)$ and thus maximize (B.4) directly. Rejecting constant terms, the maximum likelihood estimate of $\boldsymbol{\theta}$ is given by

$$
\hat{\boldsymbol{\theta}}_{M L}(\mathbf{R})=\arg \max _{\boldsymbol{\theta}}\left[\sum_{m=1}^{M} Q_{1}\left(f_{m}, \boldsymbol{\theta}\right)+\sum_{m=1}^{M} Q_{2}\left(f_{m}, \boldsymbol{\theta}\right) \cdot \sum_{l=1}^{L}\left|\mathbf{R}_{l}^{\dagger}\left(f_{m}\right) \mathbf{g}\left(f_{m}, \boldsymbol{\theta}\right)\right|^{2}\right],
$$

where

$$
\begin{aligned}
& Q_{1}\left(f_{m}, \boldsymbol{\theta}\right)=-L \ln \left(\sigma_{b}^{2}\left(f_{m}\right)\left\|\mathbf{G}\left(f_{m}, \boldsymbol{\theta}\right)\right\|^{2}+\sigma_{n}^{2}\left(f_{m}\right)\right), \\
& Q_{2}\left(f_{m}, \boldsymbol{\theta}\right)=\frac{\sigma_{b}^{2}\left(f_{m}\right)\left\|\mathbf{G}\left(f_{m}, \boldsymbol{\theta}\right)\right\|^{2}}{\sigma_{n}^{2}\left(f_{m}\right)\left(\sigma_{b}^{2}\left(f_{m}\right)\left\|\mathbf{G}\left(f_{m}, \theta\right)\right\|^{2}+\sigma_{n}^{2}\left(f_{m}\right)\right)} .
\end{aligned}
$$

Note that the norm term plays opposite roles in $Q_{1}\left(f_{m}, \boldsymbol{\theta}\right)$ and $Q_{2}\left(f_{m}, \boldsymbol{\theta}\right)$. As $\left\|\mathbf{G}\left(f_{m}, \boldsymbol{\theta}\right)\right\|^{2}$ increases, $Q_{1}\left(f_{m}, \boldsymbol{\theta}\right)$ decreases, while $Q_{2}\left(f_{m}, \boldsymbol{\theta}\right)$ increases. In addition, compared to the interference pattern, $\left\|\mathbf{G}\left(f_{m}, \boldsymbol{\theta}\right)\right\|^{2}$ usually has a much weaker dependence on $\boldsymbol{\theta}$, so the $\boldsymbol{\theta}$-dependences of $Q_{1}\left(f_{m}, \boldsymbol{\theta}\right)$ and $Q_{2}\left(f_{m}, \boldsymbol{\theta}\right)$ can often be ignored. Therefore, the MLE of $\boldsymbol{\theta}$ becomes

$$
\hat{\boldsymbol{\theta}}_{M L}(\mathbf{R})=\arg \max _{\boldsymbol{\theta}} \sum_{m=1}^{M} \sum_{l=1}^{L}\left|\mathbf{R}_{l}^{\dagger}\left(f_{m}\right) \mathbf{g}\left(f_{m}, \boldsymbol{\theta}\right)\right|^{2} .
$$

Under this approximation, (B.8) is actually independent of $\sigma_{b}^{2}\left(f_{m}\right)$ and $\sigma_{n}^{2}\left(f_{m}\right)$. 


\section{Appendix C}

\section{Error Analysis of Random Signal Parameter Estimation through Binary Hypothesis Test}

A parameter estimation problem can often be transformed to a detection problem. For example, for a parameter with $M$ discrete values, one can use an M-ary detection scheme to find its estimate. In this appendix, we study the Binary Hypothesis Tests for a parameter set with two possible (vector) values. Particularly, we are interested in performance associated with the likelihood ratio test, which is often considered as the optimum test. The derived optimum error probability plays a center role in evaluation of the Ziv-Zakai bound as well as ambiguity analysis.

To determine the value of $\boldsymbol{\theta}$, we define a binary hypotheses test by

$$
\begin{array}{ll}
H_{0}: & \boldsymbol{\theta}=\boldsymbol{\theta}_{0}, \\
H_{1}: & \boldsymbol{\theta}=\boldsymbol{\theta}_{1} .
\end{array}
$$

Given the data model in (3.28) and (3.30), the pdf under the hypothesis $H_{0}$ is 


$$
\begin{aligned}
p\left(\mathbf{R} \mid \boldsymbol{\theta}_{0}\right) & =\frac{1}{\left|\pi \mathrm{K}_{\mathbf{R}}\left(\boldsymbol{\theta}_{0}\right)\right|} \exp \left(-\mathbf{R}^{\dagger} \mathrm{K}_{\mathbf{R}}^{-1}\left(\boldsymbol{\theta}_{0}\right) \mathbf{R}\right) \\
& =\frac{1}{\prod_{m=1}^{M}\left|\pi \mathrm{K}_{\mathbf{R}}\left(f_{m}, \boldsymbol{\theta}_{0}\right)\right|^{L}} \prod_{l=1}^{L} \prod_{m=1}^{M} \exp \left(-\mathbf{R}_{l}^{\dagger}\left(f_{m}\right) \mathrm{K}_{\mathbf{R}}^{-1}\left(f_{m}, \boldsymbol{\theta}_{0}\right) \mathbf{R}_{l}\left(f_{m}\right)\right)
\end{aligned}
$$

Under the hypothesis $H_{1}$, it is

$$
\begin{aligned}
p\left(\mathbf{R} \mid \boldsymbol{\theta}_{1}\right) & =\frac{1}{\left|\pi \mathrm{K}_{\mathbf{R}}\left(\boldsymbol{\theta}_{1}\right)\right|} \exp \left(-\mathbf{R}^{\dagger} \mathrm{K}_{\mathbf{R}}^{-1}\left(\boldsymbol{\theta}_{1}\right) \mathbf{R}\right) \\
& =\frac{1}{\prod_{m=1}^{M}\left|\pi \mathrm{K}_{\mathbf{R}}\left(f_{m}, \boldsymbol{\theta}_{1}\right)\right|^{L}} \prod_{l=1}^{L} \prod_{m=1}^{M} \exp \left(-\mathbf{R}_{l}^{\dagger}\left(f_{m}\right) \mathrm{K}_{\mathbf{R}}^{-1}\left(f_{m}, \boldsymbol{\theta}_{1}\right) \mathbf{R}_{l}\left(f_{m}\right)\right)
\end{aligned}
$$

For this binary hypothesis test problem, the likelihood ratio test states [72]:

$$
l(\mathbf{R})=\ln \left[\frac{p\left(\mathbf{R} \mid \boldsymbol{\theta}_{1}\right)}{p\left(\mathbf{R} \mid \boldsymbol{\theta}_{0}\right)}\right] \stackrel{H_{1}}{\underset{H_{0}}{\gtrless}} 0,
$$

where the minimum probability of error criterion is used.

Using (C.2), (C.3), (B.2), and (B.3), we have

$$
\begin{aligned}
l(\mathbf{R})= & \sum_{m=1}^{M} Q_{1}\left(f_{m}, \boldsymbol{\theta}_{1}\right)+\sum_{m=1}^{M} Q_{2}\left(f_{m}, \boldsymbol{\theta}_{1}\right) \cdot \sum_{l=1}^{L}\left|\mathbf{R}_{l}^{\dagger}\left(f_{m}\right) \mathbf{g}\left(f_{m}, \boldsymbol{\theta}_{1}\right)\right|^{2} \\
& -\sum_{m=1}^{M} Q_{1}\left(f_{m}, \boldsymbol{\theta}_{0}\right)-\sum_{m=1}^{M} Q_{2}\left(f_{m}, \boldsymbol{\theta}_{0}\right) \cdot \sum_{l=1}^{L}\left|\mathbf{R}_{l}^{\dagger}\left(f_{m}\right) \mathbf{g}\left(f_{m}, \boldsymbol{\theta}_{0}\right)\right|^{2}
\end{aligned}
$$

where $\mathbf{g}\left(f_{m}, \boldsymbol{\theta}\right)$ is the normalized Green's function, and $Q_{1}\left(f_{m}, \boldsymbol{\theta}\right)$ and $Q_{2}\left(f_{m}, \boldsymbol{\theta}\right)$ are defined in (B.6) and (B.7), respectively.

Ignoring the $\boldsymbol{\theta}$-dependences of $Q_{1}\left(f_{m}, \boldsymbol{\theta}\right)$ and $Q_{2}\left(f_{m}, \boldsymbol{\theta}\right)$, we obtain for the $\log$ likelihood ratio

$$
l(\mathbf{R})=\sum_{m=1}^{M} \sum_{l=1}^{L}\left|\mathbf{R}_{l}^{\dagger}\left(f_{m}\right) \mathbf{g}\left(f_{m}, \boldsymbol{\theta}_{1}\right)\right|^{2}-\sum_{m=1}^{M} \sum_{l=1}^{L}\left|\mathbf{R}_{l}^{\dagger}\left(f_{m}\right) \mathbf{g}\left(f_{m}, \boldsymbol{\theta}_{0}\right)\right|^{2} .
$$

Error occurs when $l(\mathbf{R})>0$ given $H_{0}$ is true or when $l(\mathbf{R})<0$ given $H_{1}$ is true, 
leading to the following error probabilities:

$$
P_{e}\left(\boldsymbol{\theta}_{1} \mid \boldsymbol{\theta}_{0}\right)=\operatorname{Pr}\left(l(\mathbf{R})>0 \mid \boldsymbol{\theta}=\boldsymbol{\theta}_{0}\right)
$$

and

$$
P_{e}\left(\boldsymbol{\theta}_{0} \mid \boldsymbol{\theta}_{1}\right)=\operatorname{Pr}\left(l(\mathbf{R})<0 \mid \boldsymbol{\theta}=\boldsymbol{\theta}_{1}\right)
$$

Definitions are symmetrical for these two probabilities, so only the first one is derived in the sequel.

\section{C.1 Case 1: Single-Frequency Component}

This corresponds to the narrowband signal model. For a single-frequency component with multiple snapshots, the desired error probability is

$$
P_{e}\left(\boldsymbol{\theta}_{1} \mid \boldsymbol{\theta}_{0}\right)=\operatorname{Pr}\left(\sum_{l=1}^{L}\left|\mathbf{R}_{l}^{\dagger}\left(f_{0}\right) \mathbf{g}\left(f_{0}, \boldsymbol{\theta}_{1}\right)\right|^{2} \geq \sum_{l=1}^{L}\left|\mathbf{R}_{l}^{\dagger}\left(f_{0}\right) \mathbf{g}\left(f_{0}, \boldsymbol{\theta}_{0}\right)\right|^{2}\right)
$$

In this case, some closed-form expressions for $P_{e}\left(\boldsymbol{\theta}_{1} \mid \boldsymbol{\theta}_{0}\right)$ are available. We present two approaches to derive $P_{e}\left(\boldsymbol{\theta}_{1} \mid \boldsymbol{\theta}_{0}\right)$. The first one follows that for incoherent diversity reception error analysis developed by Lindsey [49]. The second one is proposed by Richmond [59].

\section{C.1.1 Lindsey's Approach}

We first develop some statistics involved. Define

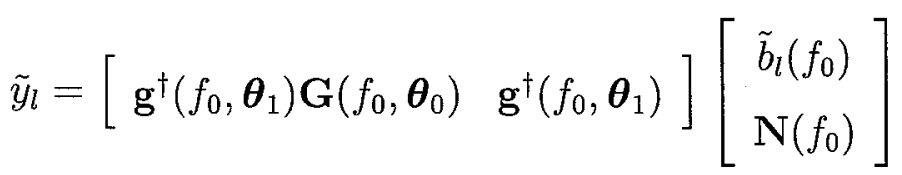

and 


$$
\tilde{x}_{l}=\left[\begin{array}{ll}
\mathbf{g}^{\dagger}\left(f_{0}, \boldsymbol{\theta}_{0}\right) \mathbf{G}\left(f_{0}, \boldsymbol{\theta}_{0}\right) & \mathbf{g}^{\dagger}\left(f_{0}, \boldsymbol{\theta}_{0}\right)
\end{array}\right]\left[\begin{array}{c}
\tilde{b}_{l}\left(f_{0}\right) \\
\mathbf{N}\left(f_{0}\right)
\end{array}\right] .
$$

Obviously, both $\tilde{y}_{l}$ and $\tilde{x}_{l}$ are zero-mean complex Gaussian random variables.

Denote the covariance matrix as follows:

$$
\Psi=\left[\begin{array}{ll}
\psi_{11} & \psi_{12} \\
\psi_{12}^{\dagger} & \psi_{22}
\end{array}\right]=E\left[\left[\begin{array}{c}
\tilde{y}_{l} \\
\tilde{x}_{l}
\end{array}\right]\left[\begin{array}{cc}
\tilde{y}_{l}^{\dagger} & \tilde{x}_{l}^{\dagger}
\end{array}\right]\right]
$$

where

$$
\begin{gathered}
\psi_{11}=E\left[\tilde{y}_{l} \tilde{y}_{l}^{\dagger}\right]=\sigma_{b}^{2}\left(f_{0}\right) \cdot\left\|\mathbf{G}\left(f_{0}, \boldsymbol{\theta}_{0}\right)\right\|^{2} \cdot\left|\mathbf{g}^{\dagger}\left(f_{0}, \boldsymbol{\theta}_{1}\right) \mathbf{g}\left(f_{0}, \boldsymbol{\theta}_{0}\right)\right|^{2}+\sigma_{n}^{2}\left(f_{0}\right), \\
\psi_{22}=E\left[\tilde{x}_{l} \tilde{x}_{l}^{\dagger}\right]=\sigma_{b}^{2}\left(f_{0}\right) \cdot\left\|\mathbf{G}\left(f_{0}, \boldsymbol{\theta}_{0}\right)\right\|^{2}+\sigma_{n}^{2}\left(f_{0}\right), \\
\psi_{12}=\psi_{21}^{\dagger}=E\left[\tilde{y}_{l} \tilde{x}_{l}^{\dagger}\right]=\mathbf{g}^{\dagger}\left(f_{0}, \boldsymbol{\theta}_{1}\right) \mathbf{g}\left(f_{0}, \boldsymbol{\theta}_{0}\right) \cdot\left(\sigma_{b}^{2}\left(f_{0}\right) \cdot\left\|\mathbf{G}\left(f_{0}, \boldsymbol{\theta}_{0}\right)\right\|^{2}+\sigma_{n}^{2}\left(f_{0}\right)\right) .
\end{gathered}
$$

We also denote the inverse of the covariance matrix as

$$
\Phi=\left[\begin{array}{ll}
\phi_{11} & \phi_{12} \\
\phi_{12}^{\dagger} & \phi_{22}
\end{array}\right]=\Psi^{-1}
$$

Obviously, for non-trivial cases, $\phi_{11}>0, \phi_{22}>0$, and $|\Phi|=\phi_{11} \phi_{22}-\left|\phi_{12}\right|^{2}>0$.

Let $y_{l}=\left|\tilde{y}_{l}\right|$ and $x_{l}=\left|\tilde{x}_{l}\right|$, i.e., the amplitude of a complex variable, then the sum terms in (C.9) can be represented as

$$
\begin{aligned}
& Y=\sum_{l=1}^{L} y_{l}^{2}, \\
& X=\sum_{l=1}^{L} x_{l}^{2} .
\end{aligned}
$$


Since $x_{l}^{2}, l=1, \ldots, L$, are statistically independent, Chi-squared distributed with two degrees of freedom, the characteristic function of $X=\sum_{l=1}^{L} x_{l}^{2}$ is the product of the individual ones. Therefore, the pdf of $X$ belongs to the Pearson Type III [3, (26.1.31)], viz.,

$$
p(X)=\left(\frac{|\Phi|}{\phi_{11}}\right)^{L} \frac{1}{(L-1) !} X^{L-1} \exp \left(-\frac{|\Phi|}{\phi_{11}} X\right)
$$

To find the conditional pdf of $Y$ given $X$, we first find $p\left(y_{l} \mid x_{l}\right)$ from $p\left(y_{l}, x_{l}\right)$ using the Bayes rule; then follow a similar procedure for $p(X)$. The resulting $p(Y \mid X)$ is

$$
p(Y \mid X)=\frac{\phi_{11}^{L}}{\left|\phi_{12}\right|^{L-1}} \exp \left(-\frac{\left|\phi_{12}\right|^{2}}{\phi_{11}} X-\phi_{11} Y\right) \cdot\left(\frac{Y}{X}\right)^{\frac{L-1}{2}} \cdot I_{L-1}\left(2\left|\phi_{12}\right| \sqrt{X Y}\right),
$$

where $I_{L-1}(\cdot)$ is the modified Bessel function of the first kind and order $L-1$.

Given $p(X)$ and $p(Y \mid X)$, the error probability in (C.9) can be expressed as

$$
P_{e}=\operatorname{Pr}(Y>X)=\int_{0}^{\infty} d X \cdot p(X) \int_{X}^{\infty} p(Y \mid X) d Y
$$

Substituting (C.20) into (C.21), we have

$$
\begin{aligned}
P_{e}= & \int_{0}^{\infty} d X \cdot p(X) \int_{X}^{\infty} d Y \\
& \cdot \frac{\phi_{11}^{L}}{\left|\phi_{12}\right|^{L-1}} \exp \left(-\frac{\left|\phi_{12}\right|^{2}}{\phi_{11}} X-\phi_{11} Y\right) \cdot\left(\frac{Y}{X}\right)^{\frac{L-1}{2}} I_{L-1}\left(2\left|\phi_{12}\right| \sqrt{X Y}\right) .
\end{aligned}
$$

Introducing a change of variable given by $z=\sqrt{2 \phi_{11} Y}$, the second integration is actually in the form of the generalization of the Marcum Q-function [37, $(2.17,2.18)]$ :

$$
\begin{aligned}
Q_{M}(\alpha, \beta) & =\int_{\beta}^{\infty} x\left(\frac{x}{\alpha}\right)^{M-1} \exp \left(-\frac{x^{2}+\alpha^{2}}{2}\right) I_{M-1}(\alpha x) d x \\
& =Q(\alpha, \beta)+\exp \left(-\frac{\alpha^{2}+\beta^{2}}{2}\right) \sum_{r=1}^{M-1}\left(\frac{\beta}{\alpha}\right)^{r} I_{r}(\alpha \beta),
\end{aligned}
$$


where $Q(\alpha, \beta)$ is known as the "Q-function" [37, (F.1, F.2)]:

$$
Q(\alpha, \beta)=\int_{\beta}^{\infty} x \exp \left(-\frac{x^{2}+\alpha^{2}}{2}\right) I_{0}(\alpha x) d x
$$

In terms of the generalization of the Marcum Q-function, the error probability is

$$
\begin{aligned}
P_{e}= & \int_{0}^{\infty} p(X) \cdot Q_{L}\left(\sqrt{\frac{2\left|\phi_{12}\right|^{2} X}{\phi_{11}}}, \sqrt{2 \phi_{11} X}\right) d X \\
= & \int_{0}^{\infty} d x \cdot p(x) \cdot\left[Q\left(\sqrt{\frac{2\left|\phi_{12}\right|^{2}}{\phi_{11}}} x, \sqrt{2 \phi_{11} x}\right)\right. \\
& \left.+\exp \left(-\left(\frac{\left|\phi_{12}\right|^{2}}{\phi_{11}}+\phi_{11}\right) x^{2}\right) \sum_{n=1}^{L-1}\left(\frac{\phi_{11}}{\left|\phi_{12}\right|}\right)^{n} I_{n}\left(2\left|\phi_{12}\right| x^{2}\right)\right] .
\end{aligned}
$$

For the second equality, we have used (C.24) and made a change of variable of $x=$ $\sqrt{X}$. Now we define $P_{e}=P_{e 1}+P_{e 2}$, and

$$
\begin{aligned}
& P_{e 1}=\int_{0}^{\infty} d x \cdot p(x) \cdot Q\left(\sqrt{\frac{2\left|\phi_{12}\right|^{2}}{\phi_{11}}} x, \sqrt{2 \phi_{11}} x\right) \\
& P_{e 2}=\sum_{n=1}^{L-1}\left(\frac{\phi_{11}}{\left|\phi_{12}\right|}\right)^{n} \int_{0}^{\infty} d x \cdot p(x) \cdot \exp \left(-\left(\frac{\left|\phi_{12}\right|^{2}}{\phi_{11}}+\phi_{11}\right) x^{2}\right) \cdot I_{n}\left(2\left|\phi_{12}\right| x^{2}\right)
\end{aligned}
$$

\section{$P_{e 1}$}

To develop $P_{e 1}$, we use the trigonometric form of $Q(\alpha, \beta)$ given below [37, (F.19)]:

$$
Q(\alpha, \beta)=\exp \left(-\frac{\alpha^{2}+\beta^{2}}{2}\right) \cdot \int_{0}^{2 \pi} \frac{1-\frac{\alpha}{\beta} \cos \theta}{1-2 \frac{\alpha}{\beta} \cos \theta+\left(\frac{\alpha}{\beta}\right)^{2}} \exp (\alpha \beta \cos \theta) \frac{d \theta}{2 \pi} .
$$

Then $P_{e 1}$ can be derived as follows:

$$
\begin{aligned}
P_{e 1}= & \left(\frac{|\Phi|}{\phi_{11}}\right)^{L} \frac{1}{2 \pi \cdot(L-1) !} \int_{0}^{2 \pi} d \theta \cdot \frac{1-\frac{\left|\phi_{12}\right|}{\phi_{11}} \cos \theta}{1-2 \frac{\left|\phi_{12}\right|}{\phi_{11}} \cos \theta+\left(\frac{\left|\phi_{12}\right|}{\phi_{11}}\right)^{2}} . \\
& \int_{0}^{\infty} d X \cdot X^{L-1} \cdot \exp \left(-\left(\phi_{11}+\phi_{22}-2\left|\phi_{12}\right| \cos \theta\right) X\right),
\end{aligned}
$$


where we have made a change of variable given by $X=x^{2}$. Also note that $\phi_{11}+$ $\phi_{22}-2\left|\phi_{12}\right| \cos \theta>0$ except for some trivial cases. Thus, the second integration with respect to $X$ can be carried out using Euler's integral [3, (6.1.1)].

Therefore, we have

$$
\begin{aligned}
P_{e 1}= & \frac{1}{2 \pi}\left(\frac{|\Phi|}{\phi_{11}\left(\phi_{11}+\phi_{22}\right)}\right)^{L} \frac{\phi_{11}^{2}}{\phi_{11}^{2}+\left|\phi_{12}\right|^{2}} \cdot \\
& \int_{0}^{2 \pi} d \theta \cdot \frac{1-\frac{\left|\phi_{12}\right|}{\phi_{11}} \cos \theta}{\left(1-\frac{2\left|\phi_{12}\right| \phi_{11}}{\phi_{11}^{2}+\left|\phi_{12}\right|^{2}} \cos \theta\right)\left(1-\frac{2\left|\phi_{12}\right|}{\phi_{11}+\phi_{22}} \cos \theta\right)^{L}} .
\end{aligned}
$$

Using a partial fraction expansion of the integrand and rearranging the result, we obtain

$$
P_{e 1}=P_{e 11}+P_{e 12}
$$

and

$$
\begin{aligned}
P_{e 11}= & \frac{1}{2} \cdot A^{L-1} \cdot \frac{\phi_{11}^{2}-\left|\phi_{12}\right|^{2}}{\phi_{11}^{2}+\left|\phi_{12}\right|^{2}} \\
& \cdot \frac{1}{2 \pi} \int_{0}^{2 \pi} d \theta \cdot \frac{1}{(1-C \cos \theta)(1-B \cos \theta)^{L-1}}, \\
P_{e 12}= & \frac{1}{2} \cdot A^{L-1} \cdot \frac{\phi_{22}-\phi_{11}}{\phi_{11}+\phi_{22}} \\
& \cdot \frac{1}{2 \pi} \int_{0}^{2 \pi} d \theta \cdot \frac{1}{(1-B \cos \theta)^{L}}
\end{aligned}
$$

where

$$
\begin{aligned}
A & =\frac{|\Phi|}{\phi_{11}\left(\phi_{11}+\phi_{22}\right)} \\
B & =\frac{2\left|\phi_{12}\right|}{\phi_{11}+\phi_{22}} \\
C & =\frac{2\left|\phi_{12}\right| \phi_{11}}{\phi_{11}^{2}+\left|\phi_{12}\right|^{2}}
\end{aligned}
$$


We now introduce a change of variable given by

$$
\cos \theta=\frac{B+\cos \omega}{1+B \cos \omega}
$$

This transformation maps $0 \leq \theta \leq 2 \pi$ one-to-one to $0 \leq \omega \leq 2 \pi$ and its Jacobian gives

$$
d \theta=\frac{\sqrt{1-B^{2}}}{1+B \cos \omega} d \omega
$$

Under this transformation, $P_{e 11}$ and $P_{e 12}$ become

$$
\begin{aligned}
P_{e 11}= & \frac{1}{2} \cdot A^{L-1} \cdot \frac{\phi_{11}^{2}-\left|\phi_{12}\right|^{2}}{\phi_{11}^{2}+\left|\phi_{12}\right|^{2}} \cdot\left(1-B^{2}\right)^{-L+\frac{3}{2}} \cdot \frac{1}{1-B C} \\
& \cdot \frac{1}{2 \pi} \int_{0}^{2 \pi} d \omega \cdot \frac{(1+B \cos \omega)^{L-1}}{1+E \cos \omega}, \\
P_{e 12}= & \frac{1}{2} \cdot A^{L-1} \cdot \frac{\phi_{22}-\phi_{11}}{\phi_{11}+\phi_{22}} \cdot\left(1-B^{2}\right)^{-L+\frac{1}{2}} \\
& \cdot \frac{1}{2 \pi} \int_{0}^{2 \pi} d \omega \cdot(1+B \cos \omega)^{L-1},
\end{aligned}
$$

where

$$
E=\frac{B-C}{1-B C}
$$

Note that $E<0$ and $|E|<1$ except for some trivial cases (e.g., perfect correlation or zero correlation). Hence, we can expand $1 /(1+E \cos \omega)$ using the Fourier series representation:

$$
\frac{1}{1+E \cos \omega}=\frac{1}{\sqrt{1-E^{2}}}+\sum_{n=1}^{\infty} \frac{2\left(\sqrt{1-E^{2}}-1\right)^{n}}{E^{n} \sqrt{1-E^{2}}} \cos n \omega
$$

The coefficients of the series are solved using the integral formula in [35, page 112 , (24)].

Based on this series representation and the results in [35, page 115, (35a)], $P_{e 11}$ is 
solved by

$$
\begin{aligned}
P_{e 11}= & \frac{1}{2} \cdot A^{L-1} \cdot \frac{\phi_{11}^{2}-\left|\phi_{12}\right|^{2}}{\phi_{11}^{2}+\left|\phi_{12}\right|^{2}} \cdot\left(1-B^{2}\right)^{-L+\frac{3}{2}} \cdot \frac{1}{1-B C} \\
& \cdot\left[\frac{1}{\sqrt{1-E^{2}}} \sum_{m=0}^{\left[\frac{L-1}{2}\right]}\left(\begin{array}{c}
L-1 \\
m
\end{array}\right)\left(\begin{array}{c}
L-1-m \\
m
\end{array}\right)\left(\frac{B}{2}\right)^{2 m}+\right. \\
& \left.\sum_{n=1}^{L-1} \frac{2\left(\sqrt{1-E^{2}}-1\right)^{n}}{E^{n} \sqrt{1-E^{2}}}\left(\frac{B}{2}\right)^{n} \sum_{m=0}^{\left[\frac{L-1-n}{2}\right]}\left(\begin{array}{c}
L-1 \\
m
\end{array}\right)\left(\begin{array}{c}
L-1-m \\
n+m
\end{array}\right)\left(\frac{B}{2}\right)^{2 m}\right] .
\end{aligned}
$$

Similarly, $P_{e 12}$ is solved as follows:

$$
\begin{aligned}
P_{e 12}= & \frac{1}{2} \cdot A^{L-1} \cdot \frac{\phi_{22}-\phi_{11}}{\phi_{11}+\phi_{22}} \cdot\left(1-B^{2}\right)^{-L+\frac{1}{2}} \\
& \cdot \sum_{m=0}^{\left[\frac{L-1}{2}\right]}\left(\begin{array}{c}
L-1 \\
m
\end{array}\right)\left(\begin{array}{c}
L-1-m \\
m
\end{array}\right)\left(\frac{B}{2}\right)^{2 m} .
\end{aligned}
$$

$\mathbf{P}_{\mathrm{e} 2}$

Making a change of variable of $X=x^{2}$ in (C.28) and rearranging the result, we obtain

$$
\begin{aligned}
& P_{e 2}= \\
& \left(\frac{|\Phi|}{\phi_{11}}\right)^{L} \frac{1}{(L-1) !} \sum_{n=1}^{L-1}\left(\frac{\phi_{11}}{\left|\phi_{12}\right|}\right)^{n} \int_{0}^{\infty} d X \cdot X^{L-1} \exp \left(-\left(\phi_{11}+\phi_{22}\right) X\right) \cdot I_{n}\left(2\left|\phi_{12}\right| X\right) .
\end{aligned}
$$

Note that for non-trivial cases, $\phi_{11}+\phi_{22}>2\left|\phi_{12}\right|>0$. Hence, $P_{e 2}$ can be further developed using the result in [75, page $385,(3)]$, [76, (4.2-17)] and [35, page 115 , (35a)]. Finally we obtain for $P_{e 2}$

$$
\begin{aligned}
P_{e 2}= & A^{L}\left(1-B^{2}\right)^{-L+\frac{1}{2}} \sum_{n=1}^{L-1}\left(\begin{array}{c}
L+n-1 \\
n
\end{array}\right)\left(\frac{\phi_{11}}{\phi_{11}+\phi_{22}}\right)^{n} \\
& \frac{1}{\left(\begin{array}{c}
L-1 \\
n
\end{array}\right)} \cdot \sum_{m=0}^{\left.\frac{L-1-n}{2}\right]}\left(\begin{array}{c}
L-1 \\
m
\end{array}\right)\left(\begin{array}{c}
L-1-m \\
n+m
\end{array}\right)\left(\frac{B}{2}\right)^{2 m} .
\end{aligned}
$$




\section{Summary}

In summary, the error probability in (C.9) is the sum of $P_{e 11}, P_{e 12}$ and $P_{e 2}$. Combining (C.44), (C.45) and (C.47) gives the following result:

$$
\begin{aligned}
P_{e}= & \left(\frac{A}{1-B^{2}}\right)^{L-1} \cdot\left[\frac{1}{2} \cdot\left(1+\frac{\phi_{22}-\phi_{11}}{\phi_{11}+\phi_{22}} \cdot \frac{1}{\sqrt{1-B^{2}}}\right)\right. \\
& \cdot \sum_{m=0}^{\left[\frac{L-1}{2}\right]}\left(\begin{array}{c}
L-1 \\
m
\end{array}\right)\left(\begin{array}{c}
L-1-m \\
m
\end{array}\right)\left(\frac{B}{2}\right)^{2 m} \\
& +\sum_{n=1}^{L-1}\left(\left(\frac{\sqrt{1-E^{2}}-1}{E} \cdot \frac{B}{2}\right)^{n}+\left(\frac{\phi_{11}}{\phi_{11}+\phi_{22}}\right)^{n} \cdot \frac{A}{\sqrt{1-B^{2}}} \cdot \frac{\left(\begin{array}{c}
L+n-1 \\
n
\end{array}\right)}{\left(\begin{array}{c}
L-1 \\
n
\end{array}\right)}\right) \\
& \cdot\left[\frac{L-1-n}{2}\right] \\
& \left.\sum_{m=0}^{L-1}\left(\begin{array}{c}
L-1 \\
m
\end{array}\right)\left(\begin{array}{c}
L-1-m \\
n+m
\end{array}\right)\left(\frac{B}{2}\right)^{2 m}\right] .
\end{aligned}
$$

Note that any sum equals to zero if the lower limit exceeds the upper limit.

\section{C.1.2 Richmond's Approach}

Lindsey's approach is quite straightforward, but not practical for more complicated problems, like the multiple-frequency problem. Richmond proposed a Chi-squared analysis method [59], which leads to simple derivations.

We now denote data matrix of snapshots by

$$
\mathbf{R}=\left[\begin{array}{llll}
\mathbf{R}_{1}\left(f_{0}\right) & \mathbf{R}_{2}\left(f_{0}\right) & \ldots & \mathbf{R}_{L}\left(f_{0}\right)
\end{array}\right]
$$

where $L$ snapshots are independent, identically distributed according to a complex Gaussian distribution.

Then the error probability in (C.9) can be written as

$$
\begin{aligned}
P_{e}\left(\boldsymbol{\theta}_{1} \mid \boldsymbol{\theta}_{0}\right) & =\operatorname{Pr}\left(\mathbf{g}^{\dagger}\left(f_{0}, \boldsymbol{\theta}_{1}\right) \operatorname{RR}^{\dagger} \mathbf{g}\left(f_{0}, \boldsymbol{\theta}_{1}\right) \geq \mathbf{g}^{\dagger}\left(f_{0}, \boldsymbol{\theta}_{0}\right) \operatorname{RR}^{\dagger} \mathbf{g}\left(f_{0}, \boldsymbol{\theta}_{0}\right)\right) \\
& =\operatorname{Pr}\left(\operatorname{Tr}\left[\operatorname{RR}^{\dagger} \mathbf{g}\left(f_{0}, \boldsymbol{\theta}_{1}\right) \mathbf{g}^{\dagger}\left(f_{0}, \boldsymbol{\theta}_{1}\right)\right] \geq \operatorname{Tr}\left[\operatorname{RR}^{\dagger} \mathbf{g}\left(f_{0}, \boldsymbol{\theta}_{0}\right) \mathbf{g}^{\dagger}\left(f_{0}, \boldsymbol{\theta}_{0}\right)\right]\right) \\
& =\operatorname{Pr}\left(\operatorname{Tr}\left[\operatorname{RR}^{\dagger}\left(\mathbf{g}\left(f_{0}, \boldsymbol{\theta}_{1}\right) \mathbf{g}^{\dagger}\left(f_{0}, \boldsymbol{\theta}_{1}\right)-\mathbf{g}\left(f_{0}, \boldsymbol{\theta}_{0}\right) \mathbf{g}^{\dagger}\left(f_{0}, \boldsymbol{\theta}_{0}\right)\right)\right] \geq 0\right) .
\end{aligned}
$$


We express each $\mathbf{R}_{l}$ as (recall that we are assuming $\boldsymbol{\theta}=\boldsymbol{\theta}_{0}$ )

$$
\mathbf{R}_{l}=\mathrm{K}_{\mathbf{R}}^{1 / 2}\left(f_{0}, \boldsymbol{\theta}_{0}\right) \mathbf{Z}_{l}
$$

This is actually a whitening operation so that each new snapshot, $\mathbf{Z}_{l}$, is complex Gaussian with identity covariance matrix. Hence, $P_{e}\left(\boldsymbol{\theta}_{1} \mid \boldsymbol{\theta}_{0}\right)$ can be expressed in terms of $\mathbf{Z}=\left[\mathbf{Z}_{1}, \ldots, \mathbf{Z}_{L}\right]$ :

$$
\begin{aligned}
P_{e}\left(\boldsymbol{\theta}_{1} \mid \boldsymbol{\theta}_{0}\right) & =\operatorname{Pr}\left(\operatorname{Tr}\left[\mathrm{K}_{\mathbf{R}}^{1 / 2} Z Z^{\dagger} K_{\mathbf{R}}^{1 / 2}\left(\mathbf{g}\left(f_{0}, \boldsymbol{\theta}_{1}\right) \mathbf{g}^{\dagger}\left(f_{0}, \boldsymbol{\theta}_{1}\right)-\mathbf{g}\left(f_{0}, \boldsymbol{\theta}_{0}\right) \mathbf{g}^{\dagger}\left(f_{0}, \boldsymbol{\theta}_{0}\right)\right)\right] \geq 0\right) \\
& =\operatorname{Pr}\left(\operatorname{Tr}\left[Z^{\dagger} K_{\mathbf{R}}^{1 / 2}\left(\mathbf{g}\left(f_{0}, \boldsymbol{\theta}_{1}\right) \mathbf{g}^{\dagger}\left(f_{0}, \boldsymbol{\theta}_{1}\right)-\mathbf{g}\left(f_{0}, \boldsymbol{\theta}_{0}\right) \mathbf{g}^{\dagger}\left(f_{0}, \boldsymbol{\theta}_{0}\right)\right) \mathrm{K}_{\mathbf{R}}^{1 / 2}\right] \geq 0\right) .
\end{aligned}
$$

Note that the above expression is available even for $L<N$, i.e., the number of snapshots is smaller than the number of sensors.

Denote by matrix eigen-decomposition

$$
\mathrm{K}_{\mathbf{R}}^{1 / 2}\left(\mathbf{g}\left(f_{0}, \boldsymbol{\theta}_{1}\right) \mathbf{g}^{\dagger}\left(f_{0}, \boldsymbol{\theta}_{1}\right)-\mathbf{g}\left(f_{0}, \boldsymbol{\theta}_{0}\right) \mathbf{g}^{\dagger}\left(f_{0}, \boldsymbol{\theta}_{0}\right)\right) \mathrm{K}_{\mathbf{R}}^{1 / 2}=\mathrm{U}^{\dagger} \Lambda U
$$

where $\mathbf{U}^{\dagger} \mathbf{U}=\mathbf{U U}^{\dagger}=\mathrm{I}$, and $\Lambda=\operatorname{diag}\left(\lambda_{1}, \lambda_{2}, 0, \ldots, 0\right)$.

Since $\mathrm{U}$ is a unitary matrix, multiplying $\mathrm{Z}$ by $\mathrm{U}$ doesn't alter the covariance and then the distribution for Gaussian model [64]. It follows that

$$
\begin{aligned}
\mathrm{UZ} & \stackrel{d}{=} \mathrm{Z} \\
\mathrm{UZZ}^{\dagger} \mathrm{U} & \stackrel{d}{=} \mathrm{ZZ}^{\dagger}
\end{aligned}
$$

Therefore, the error probability can be further derived as

$$
\begin{aligned}
P_{e}\left(\boldsymbol{\theta}_{1} \mid \boldsymbol{\theta}_{0}\right) & =\operatorname{Pr}\left(\operatorname{Tr}\left[\mathrm{ZZ}^{\dagger} \mathrm{U}^{\dagger} \Lambda \mathrm{U}\right] \geq 0\right) \\
& =\operatorname{Pr}\left(\operatorname{Tr}\left[\mathrm{UZZ}^{\dagger} \mathrm{U}^{\dagger} \Lambda\right] \geq 0\right) \\
& =\operatorname{Pr}\left(\operatorname{Tr}\left[\mathrm{ZZ}^{\dagger} \Lambda\right] \geq 0\right) .
\end{aligned}
$$


Expanding $\operatorname{Tr}\left[Z Z^{\dagger} \Lambda\right]$ explicitly, one can easily find it is a weighted sum of two independent degree- $L$ complex Chi-squared variables. Thus we have

$$
\begin{aligned}
P_{e}\left(\boldsymbol{\theta}_{1} \mid \boldsymbol{\theta}_{0}\right) & =\operatorname{Pr}\left(\lambda_{1} \cdot \chi_{1, L}^{2}+\lambda_{2} \cdot \chi_{2, L}^{2} \geq 0\right) \\
& =\operatorname{Pr}\left(\frac{\chi_{2, L}^{2}}{\chi_{1, L}^{2}} \geq-\frac{\lambda_{1}}{\lambda_{2}}\right) .
\end{aligned}
$$

Note that a positive $\lambda_{2}$ is required in deriving (C.57).

The statistics of the complex F-distribution, $F_{n_{1}, n_{2}}=\frac{\chi_{n_{1}}^{2}}{\chi_{n_{2}}^{2}}$, is well documented in Refs. [44] and [61, Appendix A]. Its cumulative distribution function is given by

$$
\operatorname{Pr}(F \leq x)=\frac{x^{n_{1}}}{(1+x)^{n_{1}+n_{2}-1}} \sum_{k=0}^{n_{2}-1}\left(\begin{array}{c}
n_{1}+n_{2}-1 \\
k+n_{1}
\end{array}\right) \cdot x^{k}
$$

Using (C.58), we finally obtain

$$
P_{e}\left(\boldsymbol{\theta}_{1} \mid \boldsymbol{\theta}_{0}\right)=1-\frac{\left(-\frac{\lambda_{1}}{\lambda_{2}}\right)^{L}}{\left(1-\frac{\lambda_{1}}{\lambda_{2}}\right)^{2 L-1}} \sum_{k=0}^{L-1}\left(\begin{array}{c}
2 L-1 \\
k+L
\end{array}\right) \cdot\left(-\frac{\lambda_{1}}{\lambda_{2}}\right)^{k}
$$

\section{Solutions to eigenvalues}

We first notice for any nonsingular matrix $\mathrm{K}$ and an arbitrary matrix $\mathrm{V}$,

$$
\left\{\lambda_{i}: \mathrm{K}^{1 / 2} \mathrm{VK}^{1 / 2}\right\}=\left\{\lambda_{i}: \mathrm{VK}\right\}
$$

This can be easily proved as follows:

$$
\begin{array}{r}
\left|\mathrm{K}^{1 / 2} \mathrm{VK}^{1 / 2}-\lambda\right| \mid=0 \\
\Longleftrightarrow\left|\mathrm{K}^{1 / 2}\right|\left|\mathrm{VK}^{1 / 2}-\lambda \mathrm{K}^{-1 / 2}\right|=0 \\
\Longleftrightarrow\left|\mathrm{VK}^{1 / 2}-\lambda \mathrm{K}^{-1 / 2}\right|\left|\mathrm{K}^{1 / 2}\right|=0 \\
\Longleftrightarrow|\mathrm{VK}-\lambda|=0 .
\end{array}
$$

Hence, if we denote 


$$
\begin{gathered}
\mathrm{S}_{1}=\mathrm{K}_{\mathbf{R}}^{1 / 2}\left(\mathbf{g}\left(f_{0}, \boldsymbol{\theta}_{1}\right) \mathbf{g}^{\dagger}\left(f_{0}, \boldsymbol{\theta}_{1}\right)-\mathbf{g}\left(f_{0}, \boldsymbol{\theta}_{0}\right) \mathbf{g}^{\dagger}\left(f_{0}, \boldsymbol{\theta}_{0}\right)\right) \mathrm{K}_{\mathbf{R}}^{1 / 2}, \\
\mathrm{~S}_{2}=\left(\mathbf{g}\left(f_{0}, \boldsymbol{\theta}_{1}\right) \mathbf{g}^{\dagger}\left(f_{0}, \boldsymbol{\theta}_{1}\right)-\mathbf{g}\left(f_{0}, \boldsymbol{\theta}_{0}\right) \mathbf{g}^{\dagger}\left(f_{0}, \boldsymbol{\theta}_{0}\right)\right) \mathrm{K}_{\mathbf{R}},
\end{gathered}
$$

then $S_{1}$ and $S_{2}$ share the same eigenvalues.

We know

$$
\begin{aligned}
\mathrm{S}_{2}= & \left(\mathbf{g}\left(f_{0}, \boldsymbol{\theta}_{1}\right) \mathbf{g}^{\dagger}\left(f_{0}, \boldsymbol{\theta}_{1}\right)-\mathbf{g}\left(f_{0}, \boldsymbol{\theta}_{0}\right) \mathbf{g}^{\dagger}\left(f_{0}, \boldsymbol{\theta}_{0}\right)\right) \\
& \left(\sigma_{b}^{2}\left(f_{0}\right) \mathbf{G}\left(f_{0}, \boldsymbol{\theta}_{0}\right) \mathbf{G}^{\dagger}\left(f_{0}, \boldsymbol{\theta}\right)+\sigma_{n}^{2}\left(f_{0}\right)\right) \\
= & \sigma_{b}^{2}\left(f_{0}\right)\left\|\mathbf{G}\left(f_{0}, \boldsymbol{\theta}_{0}\right)\right\|^{2} \mathbf{g}^{\dagger}\left(f_{0}, \boldsymbol{\theta}_{1}\right) \mathbf{g}\left(f_{0}, \boldsymbol{\theta}_{0}\right) \cdot \mathbf{g}\left(f_{0}, \boldsymbol{\theta}_{1}\right) \mathbf{g}^{\dagger}\left(f_{0}, \boldsymbol{\theta}_{0}\right) \\
& +\sigma_{n}^{2}\left(f_{0}\right) \cdot \mathbf{g}\left(f_{0}, \boldsymbol{\theta}_{1}\right) \mathbf{g}^{\dagger}\left(f_{0}, \boldsymbol{\theta}_{1}\right) \\
& -\left(\sigma_{b}^{2}\left(f_{0}\right)\left\|\mathbf{G}\left(f_{0}, \boldsymbol{\theta}_{0}\right)\right\|^{2}+\sigma_{n}^{2}\left(f_{0}\right)\right) \cdot \mathbf{g}\left(f_{0}, \boldsymbol{\theta}_{0}\right) \mathbf{g}^{\dagger}\left(f_{0}, \boldsymbol{\theta}_{0}\right)
\end{aligned}
$$

Obviously, $S_{2}$ is a rank-two matrix, and the eigenvectors corresponding to two nonzero eigenvalues must be linear combinations of $\mathbf{g}\left(f_{0}, \boldsymbol{\theta}_{0}\right)$ and $\mathbf{g}\left(f_{0}, \boldsymbol{\theta}_{1}\right)$. Thus

$$
\begin{aligned}
& \mathrm{S}_{2}\left(\mathbf{g}\left(f_{0}, \boldsymbol{\theta}_{0}\right)+c \mathbf{g}\left(f_{0}, \boldsymbol{\theta}_{1}\right)\right)=\lambda\left(\mathbf{g}\left(f_{0}, \boldsymbol{\theta}_{0}\right)+c \mathbf{g}\left(f_{0}, \boldsymbol{\theta}_{1}\right)\right) \\
& \Longrightarrow \\
& \mathbf{g}\left(f_{0}, \boldsymbol{\theta}_{1}\right) \cdot\left[\sigma_{b}^{2}\left(f_{0}\right)\left\|\mathbf{G}\left(f_{0}, \boldsymbol{\theta}_{0}\right)\right\|^{2} \mathbf{g}^{\dagger}\left(f_{0}, \boldsymbol{\theta}_{1}\right) \mathbf{g}\left(f_{0}, \boldsymbol{\theta}_{0}\right)\right. \\
& \left.\cdot\left(1+c \mathbf{g}^{\dagger}\left(f_{0}, \boldsymbol{\theta}_{0}\right) \mathbf{g}\left(f_{0}, \boldsymbol{\theta}_{1}\right)\right)+\sigma_{n}^{2}\left(f_{0}\right)\left(\mathbf{g}^{\dagger}\left(f_{0}, \boldsymbol{\theta}_{1}\right) \mathbf{g}\left(f_{0}, \boldsymbol{\theta}_{0}\right)+c\right)-\lambda c\right] \\
& \mathbf{g}\left(f_{0}, \boldsymbol{\theta}_{0}\right) \cdot\left[\left(\sigma_{b}^{2}\left(f_{0}\right)\left\|\mathbf{G}\left(f_{0}, \boldsymbol{\theta}_{0}\right)\right\|^{2}+\sigma_{n}^{2}\left(f_{0}\right)\right)\left(1+c \mathbf{g}^{\dagger}\left(f_{0}, \boldsymbol{\theta}_{0}\right) \mathbf{g}\left(f_{0}, \boldsymbol{\theta}_{1}\right)\right)+\lambda\right] \\
& =0 .
\end{aligned}
$$

The coefficients of $\mathbf{g}\left(f_{0}, \boldsymbol{\theta}_{0}\right)$ and $\mathbf{g}\left(f_{0}, \boldsymbol{\theta}_{1}\right)$ must both be zero. The value of $c$ that makes the coefficient of $\mathbf{g}\left(f_{0}, \boldsymbol{\theta}_{0}\right)$ zero is

$$
c=-\frac{1}{\mathbf{g}^{\dagger}\left(f_{0}, \boldsymbol{\theta}_{0}\right) \mathbf{g}\left(f_{0}, \boldsymbol{\theta}_{1}\right)}\left(\frac{\lambda}{\sigma_{b}^{2}\left(f_{0}\right)\left\|\mathbf{G}\left(f_{0}, \boldsymbol{\theta}_{0}\right)\right\|^{2}+\sigma_{n}^{2}\left(f_{0}\right)}+1\right) .
$$


Substitution of the above $c$ into the coefficient of $\mathbf{g}\left(f_{0}, \boldsymbol{\theta}_{1}\right)$ yields a quadratic equation of $\lambda$ :

$$
\begin{aligned}
& \lambda^{2}+\lambda \cdot \sigma_{b}^{2}\left(f_{0}\right)\left\|\mathbf{G}\left(f_{0}, \boldsymbol{\theta}_{0}\right)\right\|^{2}\left(1-\left|\mathbf{g}^{\dagger}\left(f_{0}, \boldsymbol{\theta}_{1}\right) \mathbf{g}\left(f_{0}, \boldsymbol{\theta}_{0}\right)\right|^{2}\right) \\
& -\sigma_{n}^{2}\left(f_{0}\right)\left(\sigma_{b}^{2}\left(f_{0}\right)\left\|\mathbf{G}\left(f_{0}, \boldsymbol{\theta}_{0}\right)\right\|^{2}+\sigma_{n}^{2}\left(f_{0}\right)\right)\left(1-\left|\mathbf{g}^{\dagger}\left(f_{0}, \boldsymbol{\theta}_{1}\right) \mathbf{g}\left(f_{0}, \boldsymbol{\theta}_{0}\right)\right|^{2}\right)=0 .
\end{aligned}
$$

The solution is

$$
\lambda\left(f_{0}, \boldsymbol{\theta}_{0}, \boldsymbol{\theta}_{1}\right)=\frac{1}{2} \cdot\left[-\sigma_{b}^{2}\left(f_{0}\right)\left\|\mathbf{G}\left(f_{0}, \boldsymbol{\theta}_{0}\right)\right\|^{2}\left(1-\left|\mathbf{g}^{\dagger}\left(f_{0}, \boldsymbol{\theta}_{1}\right) \mathbf{g}\left(f_{0}, \boldsymbol{\theta}_{0}\right)\right|^{2}\right) \mp \sqrt{D}\right],
$$

where

$$
\begin{aligned}
D\left(f_{0}, \boldsymbol{\theta}_{0}, \boldsymbol{\theta}_{1}\right)= & \left(\sigma_{b}^{2}\left(f_{0}\right)\left\|\mathbf{G}\left(f_{0}, \boldsymbol{\theta}_{0}\right)\right\|^{2}\left(1-\left|\mathbf{g}^{\dagger}\left(f_{0}, \boldsymbol{\theta}_{1}\right) \mathbf{g}\left(f_{0}, \boldsymbol{\theta}_{0}\right)\right|^{2}\right)+2 \sigma_{n}^{2}\left(f_{0}\right)\right)^{2} \\
& -4 \sigma_{n}^{4}\left(f_{0}\right)\left|\mathbf{g}^{\dagger}\left(f_{0}, \boldsymbol{\theta}_{1}\right) \mathbf{g}\left(f_{0}, \boldsymbol{\theta}_{0}\right)\right|^{2} .
\end{aligned}
$$

\section{C.2 Case 2: Multiple-Frequency Component}

The log likelihood ratio for multiple-frequency component in (C.6) can be rewritten as

$$
l(\mathbf{R})=\sum_{m=1}^{M} \operatorname{Tr}\left[\left(\sum_{l=1}^{L} \mathbf{R}_{l}\left(f_{m}\right) \mathbf{R}_{l}^{\dagger}\left(f_{m}\right)\right) \mathbf{B}_{m}\right]
$$

where

$$
\mathrm{B}_{m}=\mathbf{g}\left(f_{m}, \boldsymbol{\theta}_{1}\right) \mathbf{g}^{\dagger}\left(f_{m}, \boldsymbol{\theta}_{1}\right)-\mathbf{g}\left(f_{m}, \boldsymbol{\theta}_{0}\right) \mathbf{g}^{\dagger}\left(f_{m}, \boldsymbol{\theta}_{0}\right)
$$

Similar to the single-frequency case, we denote data matrix of snapshots by

$$
\mathbf{R}\left(f_{m}\right)=\left[\begin{array}{llll}
\mathbf{R}_{1}\left(f_{m}\right) & \mathbf{R}_{2}\left(f_{m}\right) & \ldots & \mathbf{R}_{L}\left(f_{m}\right)
\end{array}\right]
$$


and express $\mathrm{R}\left(f_{m}\right)$ as

$$
\mathrm{R}\left(f_{m}\right)=\mathrm{K}_{\mathbf{R}}^{1 / 2}\left(f_{m}, \boldsymbol{\theta}_{0}\right) \mathrm{Z}_{m}
$$

so that each snapshot of data matrix $Z_{m}$ is complex Gaussian with identity covariance matrix.

The log likelihood ratio can then be expressed in terms of $Z_{m}$ :

$$
\begin{aligned}
l(\mathbf{R}) & =\sum_{m=1}^{M} \operatorname{Tr}\left[\mathrm{R}\left(f_{m}\right) \mathrm{R}^{\dagger}\left(f_{m}\right) \mathrm{B}_{m}\right] \\
& =\sum_{m=1}^{M} \operatorname{Tr}\left[\mathrm{K}_{\mathbf{R}}^{1 / 2}\left(f_{m}\right) \mathrm{Z}_{m} \mathrm{Z}_{m}^{\dagger} \mathrm{K}_{\mathbf{R}}^{1 / 2}\left(f_{m}\right) \mathrm{B}_{m}\right] \\
& =\sum_{m=1}^{M} \operatorname{Tr}\left[\mathrm{Z}_{m} \mathrm{Z}_{m}^{\dagger} \mathrm{K}_{\mathbf{R}}^{1 / 2}\left(f_{m}\right) \mathrm{B}_{m} \mathrm{~K}_{\mathbf{R}}^{1 / 2}\left(f_{m}\right)\right] .
\end{aligned}
$$

Using the matrix eigen-decomposition

$$
\mathrm{K}_{\mathbf{R}}^{1 / 2}\left(f_{m}\right) \mathrm{B}_{m} \mathrm{~K}_{\mathbf{R}}^{1 / 2}\left(f_{m}\right)=\mathrm{U}_{m}^{\dagger} \Lambda_{m} \mathrm{U}_{m},
$$

where $\mathrm{U}_{m}$ is a unitary matrix, and $\Lambda_{m}=\operatorname{diag}\left(\lambda_{1 m}, \lambda_{2 m}, 0, \ldots, 0\right)$, we then have

$$
\begin{aligned}
l(\mathbf{R}) & =\sum_{m=1}^{M} \operatorname{Tr}\left[\mathrm{Z}_{m} \mathrm{Z}_{m}^{\dagger} \mathrm{U}_{m}^{\dagger} \Lambda_{m} \mathrm{U}_{m}\right] \\
& =\sum_{m=1}^{M} \operatorname{Tr}\left[\mathrm{U}_{m} \mathrm{Z}_{m} \mathrm{Z}_{m}^{\dagger} \mathrm{U}_{m}^{\dagger} \Lambda_{m}\right] .
\end{aligned}
$$

Note that (C.68, C.69) can be used to solve for the eigenvalues at each frequency.

Given the equality in distribution,

$$
\mathrm{U}_{m} \mathrm{Z}_{m} \mathrm{Z}_{m}^{\dagger} \mathrm{U}_{m}^{\dagger} \stackrel{d}{=} \mathrm{Z}_{m} \mathrm{Z}_{m}^{\dagger}
$$

it follows that 


$$
\begin{aligned}
P_{e}\left(\boldsymbol{\theta}_{1} \mid \boldsymbol{\theta}_{0}\right) & =\operatorname{Pr}\left(\sum_{m=1}^{M} \operatorname{Tr}\left[\mathrm{U}_{m} \mathrm{Z}_{m} \mathrm{Z}_{m}^{\dagger} \mathrm{U}_{m}^{\dagger} \Lambda_{m}\right] \geq 0\right) \\
& =\operatorname{Pr}\left(\sum_{m=1}^{M} \operatorname{Tr}\left[\mathrm{Z}_{m} \mathrm{Z}_{m}^{\dagger} \Lambda_{m}\right] \geq 0\right) \\
& =\operatorname{Pr}\left(\sum_{m=1}^{M}\left(\lambda_{1 m} \cdot \chi_{1 m, L}^{2}+\lambda_{2 m} \cdot \chi_{2 m, L}^{2}\right) \geq 0\right) .
\end{aligned}
$$

Now we define

$$
l^{\prime}=\sum_{m=1}^{M}\left(\lambda_{1 m} \cdot \chi_{1 m, L}^{2}+\lambda_{2 m} \cdot \chi_{2 m, L}^{2}\right)
$$

so that $P_{e}\left(\boldsymbol{\theta}_{1} \mid \boldsymbol{\theta}_{0}\right)=\operatorname{Pr}\left(l^{\prime} \geq 0\right)$. To find the pdf of $l^{\prime}$, we first observe that each Chi-squared variable is independent of the others, so the moment generating function is easily solved:

$$
\begin{aligned}
\Phi_{l^{\prime}}(s) & =E\left[\exp \left(s l^{\prime}\right)\right]=\prod_{m=1}^{M} E\left[\exp \left(s \lambda_{1 m} \cdot \chi_{1 m, L}^{2}\right)\right] \cdot E\left[\exp \left(s \lambda_{2 m} \cdot \chi_{2 m, L}^{2}\right)\right] \\
& =\prod_{m=1}^{M} \frac{1}{\left(1-s \lambda_{1 m}\right)^{L}} \cdot \frac{1}{\left(1-s \lambda_{2 m}\right)^{L}}
\end{aligned}
$$

An interesting point is that (C.80) can also be derived using some properties of the covariance matrix distribution [4]. Let us reconsider the log likelihood ratio in (C.70). Since $\mathbf{R}_{1}\left(f_{m}\right), \ldots, \mathbf{R}_{L}\left(f_{m}\right)$, are independently distributed, each according to zero-mean Gaussian with covariance matrix, $\mathrm{K}_{\mathbf{R}}\left(f_{m}\right)$, the sample covariance matrix

$$
\mathrm{A}_{m}=\sum_{l=1}^{L} \mathbf{R}_{l}\left(f_{m}\right) \mathbf{R}_{l}^{\dagger}\left(f_{m}\right)
$$

is distributed according to the Wishart distribution, $W\left(\mathrm{~K}_{\mathbf{R}}\left(f_{m}\right), L-1\right)$. Despite the complicated pdf, the Wishart distribution has a simple form for its moment generating function, that is 


$$
E\left[\exp \left(\operatorname{Tr}\left[\mathrm{A}_{m} \Xi\right]\right]=\left|1-\Xi \mathrm{K}_{\mathbf{R}}\left(f_{m}\right)\right|^{-L} .\right.
$$

Using this property, the moment generating function of $l(\mathbf{R})$ can be directly solved from (C.70) as follows:

$$
\begin{aligned}
\Phi_{l(\mathbf{R})}(s) & =E[\exp (s l(\mathbf{R}))]=E\left[\exp \left(s l\left(\mathrm{~A}_{m}, m=1, \ldots, M\right)\right)\right] \\
& =E\left[\exp \left(s \sum_{m=1}^{M} \operatorname{Tr}\left[\mathrm{A}_{m} \mathrm{~B}_{m}\right]\right)\right] \\
& =E\left[\exp \left(\sum_{m=1}^{M} \operatorname{Tr}\left[\mathrm{A}_{m} s \mathrm{~B}_{m}\right]\right)\right] \\
& =\prod_{m=1}^{M} E\left[\exp \left(\operatorname{Tr}\left[\mathrm{A}_{m} s \mathrm{~B}_{m}\right]\right)\right] \\
& =\prod_{m=1}^{M}\left|1-s \mathrm{~B}_{m} \mathrm{~K}_{\mathbf{R}}\left(f_{m}\right)\right|^{-L} \\
& =\prod_{m=1}^{M}\left[\left(1-s \lambda_{1 m}\right)\left(1-s \lambda_{2 m}\right)\right]^{-L}
\end{aligned}
$$

The fifth equality comes from the independence of $A_{m}, m=1, \ldots, M$. The sixth equality follows the approach leading to (C.82). The seventh equality is because $\mathrm{B}_{m} \mathrm{~K}_{\mathbf{R}}\left(f_{m}\right)$ has only two non-zero eigenvalues at frequency $f_{m}$.

Note that even though for $L<N, \mathrm{~A}_{m}$ does not have a density, its distribution and the moment generating function are well defined. Accordingly, the derivations in (C.83) still hold for $L<N$.

Having obtained the moment generating function, we can either derive the exact form of the desired error probability or develop some approximations for rapid computation.

\section{C.2.1 Exact Error Probability}

We first rewrite (C.80) as 


$$
\Phi_{l^{\prime}}(s)=\prod_{m=1}^{M}\left(\frac{\frac{1}{\lambda_{1 m}}}{s-\frac{1}{\lambda_{1 m}}}\right)^{L}\left(\frac{\frac{1}{\lambda_{2 m}}}{s-\frac{1}{\lambda_{2 m}}}\right)^{L}
$$

$\left\{\lambda_{1 m}, \lambda_{2 m}\right\}, m=1, \ldots, M$, are solved by (C.68, C.69). Clearly (see also (C.57)),

$$
\begin{aligned}
\lambda_{1 m} & <0 \\
\lambda_{2 m} & >0 \\
\left|\lambda_{2 m}\right| & <\left|\lambda_{1 m}\right| .
\end{aligned}
$$

Note that $s$ is a real variable, and the region of convergence [55] for $\Phi_{l^{\prime}}(s)$ is given by

$$
\frac{1}{\min \left(\lambda_{1 m}, m=1, \ldots, M\right)}<s<\frac{1}{\max \left(\lambda_{2 m}, m=1, \ldots, M\right)}
$$

$\Phi_{l^{\prime}}(s)$ has a partial-fraction expansion of the form [60]:

$$
\Phi_{l^{\prime}}(s)=\sum_{m=1}^{M} \sum_{k=1}^{L}\left[\frac{C_{m k+}}{\left(s-\frac{1}{\lambda_{2 m}}\right)^{k}}+\frac{C_{m k-}}{\left(s-\frac{1}{\lambda_{1 m}}\right)^{k}}\right]
$$

where

$$
\begin{aligned}
C_{m k+} & =\left.\frac{1}{(L-k) !}\left[\frac{\partial^{L-k}}{\partial s^{L-k}}\left(\left(s-\frac{1}{\lambda_{2 m}}\right)^{L} \Phi_{l^{\prime}}\right)\right]\right|_{s=1 / \lambda_{2 m}}, \\
C_{m k-} & =\left.\frac{1}{(L-k) !}\left[\frac{\partial^{L-k}}{\partial s^{L-k}}\left(\left(s-\frac{1}{\lambda_{1 m}}\right)^{L} \Phi_{l^{\prime}}\right)\right]\right|_{s=1 / \lambda_{1 m}} .
\end{aligned}
$$

Note that for $y=\lambda \cdot \chi_{k}^{2}$ and $\lambda>0$,

$$
\begin{aligned}
p(y)=\frac{y^{k-1} e^{-y / \lambda}}{\lambda^{k}(k-1) !} \longleftrightarrow \Phi_{y}(s)=\left(\frac{\frac{1}{\lambda}}{\frac{1}{\lambda}-s}\right)^{k} \\
\Longrightarrow(-1)^{k} \frac{y^{k-1} e^{-y / \lambda}}{(k-1) !} \longleftrightarrow\left(\frac{1}{s-\frac{1}{\lambda}}\right)^{k},
\end{aligned}
$$


and for $y=\lambda \cdot \chi_{k}^{2}$ and $\lambda<0$,

$$
\begin{gathered}
p(y)=-\frac{y^{k-1} e^{-y / \lambda}}{\lambda^{k}(k-1) !} \longleftrightarrow \Phi_{y}(s)=\left(\frac{\frac{1}{\lambda}}{\frac{1}{\lambda}-s}\right)^{k} \\
\Longrightarrow \frac{(-y)^{k-1} e^{-y / \lambda}}{(k-1) !} \longleftrightarrow\left(\frac{1}{s-\frac{1}{\lambda}}\right)^{k} .
\end{gathered}
$$

Therefore the pdf of $l^{\prime}$ is given by

$$
\begin{aligned}
& p\left(l^{\prime}\right)=\sum_{m=1}^{M} \sum_{k=1}^{L} \frac{C_{m k+}}{(k-1) !}(-1)^{k} l^{k-1} e^{-l^{\prime} / \lambda_{2 m}}, \\
& \text { for } l^{\prime}>0 \\
&=\sum_{m=1}^{M} \sum_{k=1}^{L} \frac{C_{m k-1}}{(k-1) !}\left(-l^{\prime}\right)^{k-1} e^{-l^{\prime} / \lambda_{1 m}}, \\
& \text { for } l^{\prime}<0 .
\end{aligned}
$$

Finally, the error probability is

$$
\begin{aligned}
P_{e}\left(\boldsymbol{\theta}_{1} \mid \boldsymbol{\theta}_{0}\right) & =\int_{0}^{\infty} p\left(l^{\prime}\right) d l^{\prime} \\
& =\sum_{m=1}^{M} \sum_{k=1}^{L} \frac{C_{m k+}}{(k-1) !}(-1)^{k} \int_{0}^{\infty} l^{k-1} e^{-l^{\prime} / \lambda_{2 m}} d l^{\prime} \\
& =\sum_{m=1}^{M} \sum_{k=1}^{L} C_{m k+}\left(-\lambda_{2 m}\right)^{k}
\end{aligned}
$$

\section{C.2.2 Approximation to Error Probability}

The error probability in (C.93) has a nice closed-form expression, but to evaluate it, one has to find the partial-fraction expansion coefficients, which is often timeconsuming particularly for large $M$ and $L$. In fact, people have developed some approximations for some similar error analysis problems, which we would like to apply to the problem here.

A typical approach is based on the moment generating function as developed in Refs. [24, 73]. The pdf of the log likelihood ratio is expressed in terms of the Edgeworth series with first term being Gaussian, so a hierarchy of approximations 
is obtained by retaining lower to higher-order terms. All of the coefficients of this series can be expressed in terms of a so-called semi-invariant function, $\mu(s)$, and its derivatives. $\mu(s)$ is just the logarithm of the moment generating function. Originally, it is derived using a "tilted density" for optimum likelihood ratio test: the original density is transformed so that the log likelihood ratio is nearly Gaussian. Later $\mu(s)$ is generalized to some sub-optimum test [5] so that this approximation approach can be applied to describe any random variable whose moment generating function is available.

For the problem here, $\mu(s)$ is defined by

$$
\begin{aligned}
\mu(s) & =\ln \Phi_{l}(s) \\
& =-L \sum_{m=1}^{M} \ln \left[\left(1-s \lambda_{1 m}\right)\left(1-s \lambda_{2 m}\right)\right]
\end{aligned}
$$

and we have some nice forms for its derivatives:

$$
\begin{aligned}
\dot{\mu}(s) & =L \sum_{m=1}^{M}\left[\frac{\lambda_{1 m}}{1-s \lambda_{1 m}}+\frac{\lambda_{2 m}}{1-s \lambda_{2 m}}\right], \\
\ddot{\mu}(s) & =L \sum_{m=1}^{M}\left[\left(\frac{\lambda_{1 m}}{1-s \lambda_{1 m}}\right)^{2}+\left(\frac{\lambda_{2 m}}{1-s \lambda_{2 m}}\right)^{2}\right], \\
& \ldots, \\
\mu^{(k)}(s) & =(k-1) ! L \sum_{m=1}^{M}\left[\left(\frac{\lambda_{1 m}}{1-s \lambda_{1 m}}\right)^{k}+\left(\frac{\lambda_{2 m}}{1-s \lambda_{2 m}}\right)^{k}\right] .
\end{aligned}
$$

The error probability, $P_{e}\left(\boldsymbol{\theta}_{1} \mid \boldsymbol{\theta}_{0}\right)=\operatorname{Pr}(l>0)$, can then be expressed in terms of $\mu(s)$ and its derivatives. Detailed derivations are given in Ref. [73] and only the related approximations are stated here.

First, $s$ is solved by letting $\dot{\mu}(s)=0$, that is

$$
\sum_{m=1}^{M}\left[\frac{\lambda_{1 m}}{1-s \lambda_{1 m}}+\frac{\lambda_{2 m}}{1-s \lambda_{2 m}}\right]=0
$$

Except for some extremely simple cases (say, $M=1$ ), the above equation is solved numerically. Note that there are $2 M-1$ roots and $2 M$ poles. When $s$ approaches a 
pole from the negative side (i.e., $s$ is smaller), the function in (C.98) goes to infinity; when $s$ approaches a pole from the positive side, this function goes to minus-infinity. Hence, between any two adjacent poles, the left-hand-side of (C.98) changes its sign once and only once, and there is a zero within each of $2 M-1$ such sub-intervals. To satisfy the convergence condition in (C.86), only the zero falling between

$$
\frac{1}{\min \left(\lambda_{1 m} m=1, \ldots, M\right)}
$$

and

$$
\frac{1}{\max \left(\lambda_{2 m} m=1, \ldots, M\right)}
$$

is the desired solution. We denote this solution as $s_{m}$.

The first term in the series is given by

$$
P_{e}^{[1]}\left(\boldsymbol{\theta}_{1} \mid \boldsymbol{\theta}_{0}\right)=\exp \left(\mu\left(s_{m}\right)+\frac{s_{m}^{2} \ddot{\mu}\left(s_{m}\right)}{2}\right) \operatorname{erfc}\left(s_{m} \sqrt{\ddot{\mu}\left(s_{m}\right)}\right)
$$

where $\operatorname{erfc}(\cdot)$ is the complement error function defined by

$$
\operatorname{erfc}(x) \equiv \int_{x}^{\infty} \frac{1}{\sqrt{2 \pi}} \exp \left(-\frac{x^{2}}{2}\right) d x
$$

The second term in the approximation is given by

$$
P_{e}^{[2]}\left(\boldsymbol{\theta}_{1} \mid \boldsymbol{\theta}_{0}\right)=-\frac{s_{m}^{3} \mu^{(3)}\left(s_{m}\right)}{6} P_{e}^{[1]}\left(\boldsymbol{\theta}_{1} \mid \boldsymbol{\theta}_{0}\right)+\frac{1-s_{m}^{2} \ddot{\mu}\left(s_{m}\right)}{6 \sqrt{2 \pi}} \frac{\mu^{(3)}\left(s_{m}\right)}{\left(\ddot{\mu}\left(s_{m}\right)\right)^{3 / 2}} \exp \left(\mu\left(s_{m}\right)\right)
$$

Note that the log likelihood ratio is often a sum of many independent random variables. In this case, the first-order approximation is good enough.

Finally we should point out that for the case with coherent broadband signal, as long as the frequency coherence is available, the above approach can still be applied to obtain the likelihood function statistics and then the related performance measures. 


\section{Appendix D}

\section{Issues on Signal-to-Noise Ratio}

Consider the passive source localization problem. According to the passive sonar equation [74], the signal-to-noise ratio at each sensor is given by

$$
\mathrm{SNR}=\mathrm{SL}-\mathrm{TL}-\mathrm{NL}
$$

where

$\mathrm{SL}$ is the Source Level, in $\mathrm{dB}$ re $1 \mu \mathrm{Pa}$ at $1 \mathrm{~m}$;

$\mathrm{TL}$ is the Transmission Loss, in $\mathrm{dB}$ re at $1 \mathrm{~m}$; and

NL is the Noise Level, in $\mathrm{dB}$ re $1 \mu \mathrm{Pa}$.

It is often measured on a per-Hertz basis. For example, typical shallow water noise spectrum level ranges from 70 to $90 \mathrm{~dB}$ re $1 \mu \mathrm{Pa}$ per Hertz; for the sound propagation to about 4 kilometers, the transmission loss is about $60 \mathrm{~dB}$ re at $1 \mathrm{~m}$; thus, for a source spectrum level of $140 \mathrm{~dB}$ re $1 \mu \mathrm{Pa}$ per Hertz at $1 \mathrm{~m}$, the SNR varies from -10 to 10 $\mathrm{dB}$.

In the context of this thesis, we need to specify the signal term and the noise term for a given SNR. Note that

$$
\mathrm{SL}=171+10 \log _{10} P_{s}
$$


and

$$
\mathrm{NL}=171+10 \log _{10} P_{n},
$$

where $P_{s}$ and $P_{n}$, both measured in watts, denote the signal power and the noise power, respectively. For a stationary random source process, the ratio of the signal power and the noise power in the same frequency band is concerned [74], and the signal power is given by

$$
P_{s}=\int_{\Delta f} S_{b}(f) d f \approx S_{b}(f) \cdot \Delta f
$$

where $\Delta f$ (in Hertz) is the effective bandwidth of the source process, and the approximation is due to the narrowband assumption. Thus, the signal power spectral density (in watt per Hertz) can be approximated by

$$
S_{b}(f) \approx \frac{10^{(\mathrm{SL}-171) / 10}}{\Delta f}
$$

The noise power spectral density (in watt per Hertz) is approximated in the same way:

$$
S_{n}(f) \approx \frac{10^{(\mathrm{NL}-171) / 10}}{\Delta f}=\frac{10^{(\mathrm{SL}-\mathrm{TL}-\mathrm{SNR}-171) / 10}}{\Delta f}
$$

where the sonar equation in (D.1) is used. Combining (D.5) and (D.6), we have

$$
\frac{S_{n}(f)}{S_{b}(f)}=10^{-\mathrm{TL}-\mathrm{SNR} / 10}
$$

In this thesis, to simplify the calculation, we set $S_{b}(f)=1$, and then solve $S_{n}(f)$ using (D.7) for a chosen set of SNR's. The covariance matrix in (3.29) is then available.

For the continuous tone radiation, the ratio of the signal energy in receiver band and the noise power in $1-\mathrm{Hz}$ band is concerned [74]. $\Delta f$ is now determined from the FFT duration as well as the window function applied, but some similar expression in (D.7) connecting the signal level and the noise level is again available. 
The SNR defined in (D.1) is the input, sensor-averaged SNR. The transmission loss is the mean across the sensor array, i.e.,

$$
\mathrm{TL}=10 \log _{10} \frac{1}{\|\mathbf{G}(f, \boldsymbol{\theta})\|^{2} / N}
$$

where $N$ is the sensor number. For multiple tone signals, the transmission loss varies from frequency to frequency, but this variation is small for typical frequency band used. Instead of computing $S_{n}(f)$ for each tone, we compute an (incoherently) averaged transmission loss across frequencies (as well as sensors), and then use one $S_{n}(f)$ for all frequency components. This is consistent with the definition in SWellEX experimental data processing [17], which computes the input SNR using individual FFT bins for both signal plus noise and noise and then averages across frequencies and sensors. Similarly, for a discrete set of parameters, the transmission loss is further (incoherently) averaged across the parameter interval.

To evaluate the output SNR, we need to include the array gain, which is

$$
\mathrm{AG}=10 \log _{10} N
$$

For most examples in this thesis, $N=16$, and thus $\mathrm{AG} \approx 12 \mathrm{~dB}$. If we use multiple $(M)$ independent frequencies or $(L)$ snapshots, we need to add another $5 \log _{10} M$ or $5 \log _{10} L \mathrm{~dB}$ to the output SNR for typical input SNR levels [74]. For example, given that we have 16 sensors and 10 snapshots centered at a single frequency, for an input SNR of $0 \mathrm{~dB}$, the output SNR is about $(12+5=) 17 \mathrm{~dB}$. 


\section{Bibliography}

[1] C. K. Abbey and J. L. Denny. The Barankin bound: instability in certain estimation problems. SPIE, 2708:53-60, 1996.

[2] J. S. Abel. A bound on mean-square-estimate error. IEEE Trans. Information Theory, pages 1675-1680, May 1993.

[3] M. Abramowitz and I. E. Stegun. Handbook of Mathematical Functions. National Bureau of Standards, Washington, 1972.

[4] T. W. Anderson. An Introduction to Multivariate Statistical Analysis. John Wiley \& Sons, New York, 1984.

[5] A. B. Baggeroer. Confidence interval determination for spectral estimates using "tilted densities". In Proc. ICASSP 83, pages 1454-1457, Boston, 1983.

[6] A. B. Baggeroer. Matched field processing: status at 2000 and where to now. In IEEE Sensor Array and Multichannel Signal Processing Workshop, Cambridge, 2000.

[7] A. B. Baggeroer and H. Cox. Passive sonar limits upon nulling multiple moving ships with large aperture arrays. In Conference Record of the Thirty-Third Asilomar Conference on Signals, Systems, and Computers, pages 103-108, 1999.

[8] A. B. Baggeroer, W. A. Kuperman, and P. N. Mikhalevsky. An overview of matched field methods in ocean acoustics. IEEE J. Oceanic Engineering, 18:401424, October 1993. 
[9] A. B. Baggeroer, W. A. Kuperman, and H. Schmidt. Matched field processing: Source localization in correlated noise as an optimum parameter estimation problem. J. Acoust. Soc. Amer., 83:571-587, February 1988.

[10] A. B. Baggeroer and H. Schmidt. Parameter estimation theory bounds and the accuracy of full field inversions. Full Field Inversion Methods in Ocean and Seismo-Acoustics, pages 79-84, 1995.

[11] E. W. Barankin. Locally best unbiased estimators. Annals of Mathematical Statistics, 20:477-501, 1949.

[12] P. A. Baxley, N. O. Booth, and W. S. Hodgkiss. Matched-field replica model optimization and bottom property inversion in shallow water. J. Acoust. Soc. Amer., 107:1301-1323, March 2000.

[13] K. L. Bell. Performance Bounds in Parameter Estimation with Application to Bearing Estimation. PhD thesis, George Mason University, Fairfax, VA, 1995.

[14] K. L. Bell, Y. Ephraim, and H. L. Van Trees. Explicit Ziv-Zakai lower bound for bearing estimation. IEEE Trans. Signal Processing, 44:2810-2824, November 1996.

[15] K. L. Bell, Y. Steinberg, Y. Ephraim, and H. L. Van Trees. Extended Ziv-Zakai lower bound for vector parameter estimation. IEEE Trans. Information Theory, 43:624-637, March 1997.

[16] S. Bellini and G. Tartara. Bounds on error in signal parameter estimation. IEEE Trans. Communication, 22:340-342, March 1974.

[17] N. O. Booth, P. A. Baxley, J. A. Rice, P. W. Schey, W. S. Hodgkiss, G. L. D'Spain, and J. J. Murray. Source localization with broad-band matched-field processing in shallow water. IEEE J. Oceanic Engineering, 21:402-412, October 1996. 
[18] V. V. Borodin and G. R. Minasian. Statistical approach to ocean acoustic tomography, Cramer-Rao bounds for accuracy of sound-speed field reconstruction. Full Field Inversion Methods in Ocean and Seismo-Acoustics, pages 91-95, 1995.

[19] V. V. Borodin and G. R. Minasian. Statistical formulation of ray, interference and mode acoustic tomography and its potential accuracy evaluation. Full Field Inversion Methods in Ocean and Seismo-Acoustics, pages 97-101, 1995.

[20] L. M. Brekhovskikh. Waves in Layered Media. Academic Press, New York, 1980.

[21] D. G. Chapman. Minimum variance estimation without regularity assumptions. Annals of Mathematical Statistics, 22:581-586, 1951.

[22] D. Chazan, M. Zakai, and J. Ziv. Improved lower bounds on signal parameter estimation. IEEE Trans. Information Theory, 21:90-93, January 1975.

[23] E. K.P. Chong and S. H. Zak. An Introduction to Optimization. New York: Wiley, 1996.

[24] L. D. Collins. Asymptotic Approximation to the Error Probability for Detecting Gaussian Signals. Sc.D. dissertation, Massachusetts Institute of Technology, Cambridge, MA, 1968.

[25] M. D. Collins. User's Guide for RAM Version 1.0 and 1.0p. Naval Research Laboratory, Washington, DC, 1998.

[26] M. D. Collins, W. A. Kuperman, and H. Schmidt. Nonlinear inversion for oceanbottom properties. J. Acoust. Soc. Amer., 92:2770-2783, November 1992.

[27] P. M. Daly. Cramer-rao bounds for matched field tomography and ocean acoustic tomography. Master's thesis, Massachusetts Institute of Technology, Cambridge, MA, 1997.

[28] P. M. Daly. Stochastic Matched Field Processing for Localization and Nulling of Acoustic Sources. Sc.D. dissertation, Massachusetts Institute of Technology, Cambridge, MA, 2000. 
[29] D. F. DeLong. Use of the Weiss-Weinstein bound to compare the directionfinding performance of sparse arrays. Technical Report 982, MIT Lincoln Laboratory, Lexington, MA, 1993.

[30] G. L. D'Spain, J. J. Murray, W. S. Hodgkiss, N. O. Booth, and P. W. Schey. Mirages in shallow water matched field processing. J. Acoust. Soc. Amer., 105:32453265, June 1999.

[31] C. Feuillade, D. R. Del Balzo, and M. M. Rowe. Environmental mismatch in shallow-water matched-field processing: Geoacoustic parameter variability. $J$. Acoust. Soc. Amer., 85:2354-2364, June 1989.

[32] G. V. Frisk. Ocean and Seabed Acoustics. Prentice Hall, Englewood Cliffs, New Jersey, 1994.

[33] P. Gerstoft. Inversion of seismoacoustic data using genetic algorithms and $a$ posteriori probability distributions. J. Acoust. Soc. Amer., 95:770-782, February 1994.

[34] D. F. Gingras. Methods for predicting the sensitivity of matched-field processors to mismatch. J. Acoust. Soc. Amer., 86:1940-1949, November 1989.

[35] W. Grobner and N. Hofreiter. Integraltafel: Part II. Springer-Verlag, Vienna, Austria, 1958.

[36] E. L. Hamilton. Geoacoustic modeling of the sea floor. J. Acoust. Soc. Amer., 68:1313-1340, November 1980.

[37] C. W. Helstrom. Statistical Theory of Signal Detection. Pergamon Press Ltd., Oxford, England, 1968.

[38] J. Herman, R. Kucera, and J. Simsa. Equations and Inequalities. Springer-Verlag, New York, 2000.

[39] J. P. Ianniello. Recent developments in sonar signal processing. IEEE Signal Processing Magazine, 15:27-40, July 1998. 
[40] I. A. Ibragimov and R. Z. Has'minskii. Statistical Estimation: Asymptotic Theory. Springler-Verlag, New York, 1981.

[41] F. B. Jensen and M. C. Ferla. SNAP: The SACLANTCEN Normal-Mode Acoustic Propagation Model. SACLANT ASW Research Center, La Spezia, Italy, 1979.

[42] F. B. Jensen, W. A. Kuperman, M. B. Porter, and H. Schmidt. Computational Ocean Acoustics. American Institute of Physics Press, New York, 1994.

[43] S. M. Kay. Fundamentals of Statistical Signal Processing: Estimation Theory. PTR Prentice Hall, Englewood Cliffs, NJ, 1993.

[44] E. J. Kelly and K. Forsythe. Adaptive detection and parameter estimation for multidimensional signal models. Technical Report 848, MIT Lincoln Laboratory, Lexington, MA, 1989.

[45] R. T. Kessel. A mode-based measure of field sensitivity to geoacoustic parameters in weakly range-dependent environments. J. Acoust. Soc. Amer., 105:122-129, January 1999.

[46] J. L. Krolik. Matched-field minimum variance beamforming in a random ocean channel. J. Acoust. Soc. Amer., 92:1408-1419, September 1992.

[47] J. L. Krolik and S. Narasimhan. Performance bounds on acoustic thermometry of ocean climate in the presence of mesoscale sound-speed variability. J. Acoust. Soc. Amer., 99:254-265, January 1996.

[48] E. L. Lehmann. Theory of Point Estimation. Springler-Verlag, New York, 1983.

[49] W. C. Lindsey. Error probability for incoherent diversity reception. IEEE Trans. Information Theory, pages 491-499, October 1965.

[50] N. C. Makris. Parameter resolution bounds that depend on sample size. $J$. Acoust. Soc. Amer., 99:2851-2861, May 1996.

[51] Z.-H. Michalopoulou and M. B. Porter. Matched-field processing for broad-band source localization. IEEE J. Oceanic Engineering, 21:384-392, October 1996. 
[52] W. Munk, P. Worcester, and C. Wunsch. Ocean Acoustic Tomography. Cambridge University, Cambridge, England, 1995.

[53] E. Naftali and N. C. Makris. Necessary conditions for a maximum likelihood estimate to become asymptotically unbiased and attain the Cramer-Rao lower bound, Part I: General approach with an application to time-delay and Doppler shift estimation. J. Acoust. Soc. Amer., 2001. In press.

[54] S. Narasimhan and J. L. Krolik. Fundamental limits on acoustic source range estimation performance in uncertain ocean channels. J. Acoust. Soc. Amer., 97:215-226, January 1995.

[55] A. V. Oppenheim and A. S. Willsky. Signals and Systems. Prentice Hall, Upper Saddle River, New Jersey, 1997.

[56] M. B. Porter. The KRAKEN Normal Mode Program. Naval Research Laboratory, Washington, DC, 1992.

[57] S. D. Rajan, J. F. Lynch, and G. V. Frisk. Perturbative inversion methods for obtaining bottom geoacoustic parameters in shallow water. J. Acoust. Soc. Amer., 82:998-1017, September 1987.

[58] J. Rice and J. Determan. SWellEX-3 Data Report. NCCOSC RDT\&E Division, Code 541, 1995.

[59] Christ D. Richmond. Personal Communications.

[60] Christ D. Richmond. Performance of a class of adaptive detection algorithms in nonhomogeneous environments. IEEE Trans. Signal Processing, 48:1248-1262, May 1995.

[61] Christ D. Richmond. Performance of the adaptive sidelobe blanker detection algorithms in homogeneous environments. IEEE Trans. Signal Processing, 48:1235-1247, May 1995. 
[62] H. Schmidt. OASES Version 2.1 User Guide and Reference Manual. MIT Ocean Acoustics Laboratory, Cambridge, MA, 1999.

[63] H. Schmidt and A. B. Baggeroer. Physics-imposed resolution and robustness issues in seismo-acoustic parameter inversion. Full Field Inversion Methods in Ocean and Seismo-Acoustics, pages 85-90, 1995.

[64] Allan Steinhardt. Adaptive multisensor detection. In Simon Haykin and Allan Steinhardt, editors, Adaptive Radar Detection and Estimation, chapter 3, pages 126-137. John Wiley \& Sons, New York, 1992.

[65] J. Tabrikian, G. S. Fostick, and H. Messer. Detection of environmental mismatch in a shallow water waveguide. IEEE Trans. Signal Processing, 47:2181-2190, August 1999.

[66] J. Tabrikian and J. L. Krolik. Barankin bounds for source localization in an uncertain ocean environment. IEEE Trans. Signal Processing, 47:2917-2927, November 1999.

[67] J. Tabrikian, J. L. Krolik, and H. Messer. Robust maximum-likelihood source localization in an uncertain shallow-water waveguide. J. Acoust. Soc. Amer., 101:241--249, January 1997.

[68] A. M. Thode, W. A. Kuperman, G. L. D'Spain, and W. S. Hodgkiss. Localization using Bartlett matched-field processor sidelobes. J. Acoust. Soc. Amer., 107:278286, January 2000.

[69] A. M. Thode, E. Naftali, I. Ingram, P. Ratilal, and N. C. Makris. Necessary conditions for a maximum likelihood estimate to become asymptotically unbiased and attain the Cramer-Rao lower bound, Part II: Range and depth localization of a sound source in an ocean waveguide. J. Acoust. Soc. Amer., 2001. In press.

[70] A. Tolstoy. Sensitivity of matched field processing to sound-speed profile mismatch for vertical arrays in a deep water Pacific environment. J. Acoust. Soc. Amer., 85:2394-2404, June 1989. 
[71] A. Tolstoy, O. Diachok, and L. N. Frazer. Acoustic tomography via matched field processing. J. Acoust. Soc. Amer., 89:1119-1127, March 1991.

[72] H. L. Van Trees. Detection, Estimation, and Modulation Theory, Part I. John Wiley \& Sons, New York, 1968.

[73] H. L. Van Trees. Detection, Estimation, and Modulation Theory, Part III. John Wiley \& Sons, New York, 1971.

[74] R. J. Urick. Principles of Underwater Sound. McGraw-Hill Book Company, New York, 1983.

[75] G. N. Watson. A Treatise on The Theory of Bessel Functions. Cambridge University Press, New York, 1966.

[76] N. Wax. Selected Papers on Noise and Stochastic Processes. Dover Publications, Inc., New York, 1954.

[77] E. Weinstein and A. J. Weiss. Fundamental limitations in passive time delay estimation-Part II: Wide-band systems. IEEE Trans. Acoustics, Speech, and Signal Processing, 32:1064-1078, May 1984.

[78] E. Weinstein and A. J. Weiss. A general class of lower bounds in parameter estimation. IEEE Trans. Information Theory, 34:338-342, March 1988.

[79] A. J. Weiss. Fundamental Bounds in Parameter Estimation. PhD thesis, TelAviv University, Tel-Aviv, Israel, 1985.

[80] A. J. Weiss and E. Weinstein. Fundamental limitations in passive time delay estimation-Part I: Narrow-band systems. IEEE Trans. Acoustics, Speech, and Signal Processing, 31:472-486, February 1983.

[81] A. S. Willsky and G. W. Wornell. 6.432 Stochastic Processes, Detection, and Estimation: Notes. Massachusetts Institute of Technology, Cambridge, MA, 1998. 
[82] A. Zeira and P. M. Schultheiss. Realizable lower bounds for time delay estimation. IEEE Trans. Signal Processing, 41:3102-3113, November 1993.

[83] A. Zeira and P. M. Schultheiss. Realizable lower bounds for time delay estimation: Part 2 - Threshold phenomena. IEEE Trans. Signal Processing, 42:1001-1007, May 1994.

[84] J. Ziv and M. Zakai. Some lower bounds on signal parameter estimation. IEEE Trans. Information Theory, 15:386-391, May 1969. 